\title{
The clinical and cost-effectiveness of
} left ventricular assist devices for end-stage heart failure: a systematic review and economic evaluation

AJ Clegg, DA Scott, E Loveman, J Colquitt, J Hutchinson, P Royle and J Bryant

November 2005

Health Technology Assessment NHS R\&D HTA Programme

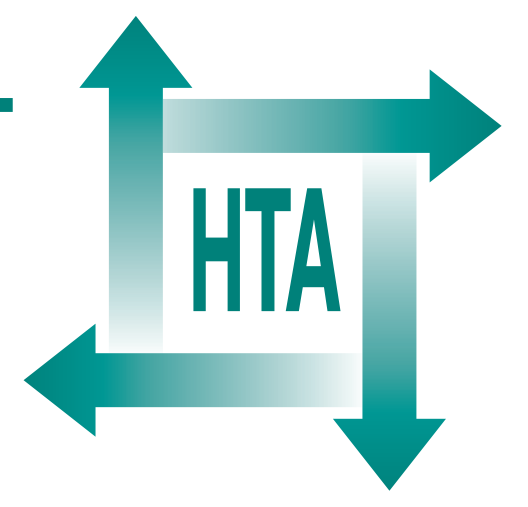




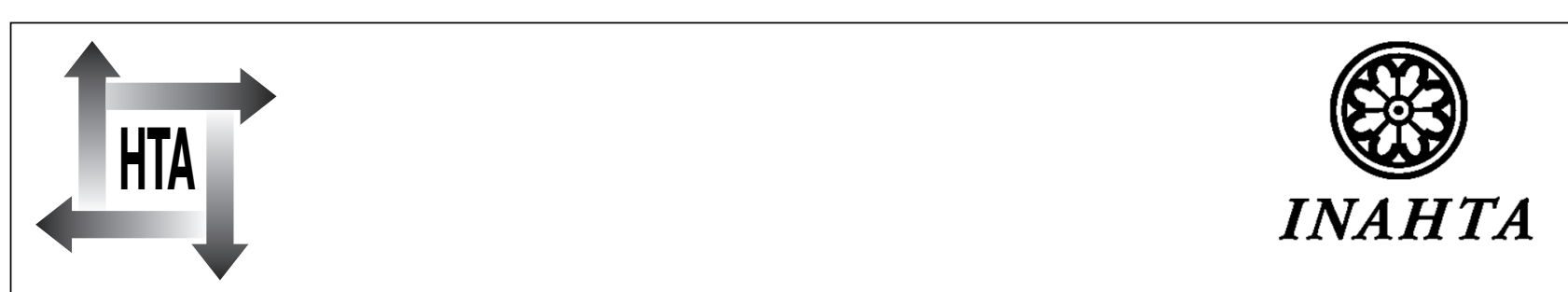

How to obtain copies of this and other HTA Programme reports.

An electronic version of this publication, in Adobe Acrobat format, is available for downloading free of charge for personal use from the HTA website (http://www.hta.ac.uk). A fully searchable CD-ROM is also available (see below).

Printed copies of HTA monographs cost $£ 20$ each (post and packing free in the UK) to both public and private sector purchasers from our Despatch Agents.

Non-UK purchasers will have to pay a small fee for post and packing. For European countries the cost is $£ 2$ per monograph and for the rest of the world $£ 3$ per monograph.

You can order HTA monographs from our Despatch Agents:

- fax (with credit card or official purchase order)

- post (with credit card or official purchase order or cheque)

- phone during office hours (credit card only).

Additionally the HTA website allows you either to pay securely by credit card or to print out your order and then post or fax it.

Contact details are as follows:

HTA Despatch

c/o Direct Mail Works Ltd

4 Oakwood Business Centre

Downley, HAVANT PO9 2NP, UK

Email: orders@hta.ac.uk

Tel: 02392492000

Fax: 02392478555

Fax from outside the UK: +442392478555

NHS libraries can subscribe free of charge. Public libraries can subscribe at a very reduced cost of $£ 100$ for each volume (normally comprising 30-40 titles). The commercial subscription rate is $£ 300$ per volume. Please see our website for details. Subscriptions can only be purchased for the current or forthcoming volume.

\section{Payment methods}

Paying by cheque

If you pay by cheque, the cheque must be in pounds sterling, made payable to Direct Mail Works Ltd and drawn on a bank with a UK address.

Paying by credit card

The following cards are accepted by phone, fax, post or via the website ordering pages: Delta, Eurocard, Mastercard, Solo, Switch and Visa. We advise against sending credit card details in a plain email.

Paying by official purchase order

You can post or fax these, but they must be from public bodies (i.e. NHS or universities) within the UK. We cannot at present accept purchase orders from commercial companies or from outside the UK.

\section{How do I get a copy of HTA on CD?}

Please use the form on the HTA website (www.hta.ac.uk/htacd.htm). Or contact Direct Mail Works (see contact details above) by email, post, fax or phone. HTA on CD is currently free of charge worldwide.

The website also provides information about the HTA Programme and lists the membership of the various committees. 


\title{
The clinical and cost-effectiveness of left ventricular assist devices for end-stage heart failure: a systematic review and economic evaluation
}

\author{
AJ Clegg, , ${ }^{*}$ DA Scott, ${ }^{2}$ E Loveman, ' J Colquitt, \\ J Hutchinson, ${ }^{2}$ P Royle ${ }^{3}$ and J Bryant ${ }^{1}$ \\ I Southampton Health Technology Assessments Centre, Wessex Institute \\ for Health Research and Development, University of Southampton, UK \\ ${ }^{2}$ Fourth Hurdle Consulting Ltd, London, UK \\ ${ }^{3}$ University of Aberdeen, UK \\ * Corresponding author
}

Declared competing interests of authors: none

Published November 2005

This report should be referenced as follows:

Clegg AJ, Scott DA, Loveman E, Colquitt J, Hutchinson J, Royle P, et al. The clinical and cost-effectiveness of left ventricular assist devices for end-stage heart failure: a systematic review and economic evaluation. Health Technol Assess 2005;9(45).

Health Technology Assessment is indexed and abstracted in Index Medicus/MEDLINE, Excerpta Medica/EMBASE and Science Citation Index Expanded (SciSearch ${ }^{\circledR}$ ) and Current Contents ${ }^{\circledR} /$ Clinical Medicine. 


\section{NHS R\&D HTA Programme}

$\mathrm{T}$ he research findings from the NHS R\&D Health Technology Assessment (HTA) Programme directly influence key decision-making bodies such as the National Institute for Health and Clinical Excellence (NICE) and the National Screening Committee (NSC) who rely on HTA outputs to help raise standards of care. HTA findings also help to improve the quality of the service in the NHS indirectly in that they form a key component of the 'National Knowledge Service' that is being developed to improve the evidence of clinical practice throughout the NHS.

The HTA Programme was set up in 1993. Its role is to ensure that high-quality research information on the costs, effectiveness and broader impact of health technologies is produced in the most efficient way for those who use, manage and provide care in the NHS. 'Health technologies' are broadly defined to include all interventions used to promote health, prevent and treat disease, and improve rehabilitation and long-term care, rather than settings of care.

The HTA Programme commissions research only on topics where it has identified key gaps in the evidence needed by the NHS. Suggestions for topics are actively sought from people working in the NHS, the public, service-users groups and professional bodies such as Royal Colleges and NHS Trusts.

Research suggestions are carefully considered by panels of independent experts (including service users) whose advice results in a ranked list of recommended research priorities. The HTA Programme then commissions the research team best suited to undertake the work, in the manner most appropriate to find the relevant answers. Some projects may take only months, others need several years to answer the research questions adequately. They may involve synthesising existing evidence or conducting a trial to produce new evidence where none currently exists.

Additionally, through its Technology Assessment Report (TAR) call-off contract, the HTA Programme is able to commission bespoke reports, principally for NICE, but also for other policy customers, such as a National Clinical Director. TARs bring together evidence on key aspects of the use of specific technologies and usually have to be completed within a short time period.

\section{Criteria for inclusion in the HTA monograph series}

Reports are published in the HTA monograph series if (1) they have resulted from work commissioned for the HTA Programme, and (2) they are of a sufficiently high scientific quality as assessed by the referees and editors.

Reviews in Health Technology Assessment are termed 'systematic' when the account of the search, appraisal and synthesis methods (to minimise biases and random errors) would, in theory, permit the replication of the review by others.

The research reported in this monograph was commissioned by the HTA Programme as project number $01 / 12 / 02$. The contractual start date was in June 2002. The draft report began editorial review in June 2004 and was accepted for publication in February 2005. As the funder, by devising a commissioning brief, the HTA Programme specified the research question and study design. The authors have been wholly responsible for all data collection, analysis and interpretation, and for writing up their work. The HTA editors and publisher have tried to ensure the accuracy of the authors' report and would like to thank the referees for their constructive comments on the draft document. However, they do not accept liability for damages or losses arising from material published in this report.

The views expressed in this publication are those of the authors and not necessarily those of the HTA Programme or the Department of Health.

Editor-in-Chief:

Professor Tom Walley

Series Editors:

Dr Peter Davidson, Dr Chris Hyde, Dr Ruairidh Milne,

Dr Rob Riemsma and Dr Ken Stein

Managing Editors: Sally Bailey and Sarah Llewellyn Lloyd

ISSN 1366-5278

\section{(c) Queen's Printer and Controller of HMSO 2005}

This monograph may be freely reproduced for the purposes of private research and study and may be included in professional journals provided that suitable acknowledgement is made and the reproduction is not associated with any form of advertising.

Applications for commercial reproduction should be addressed to NCCHTA, Mailpoint 728, Boldrewood, University of Southampton, Southampton, SOI6 7PX, UK.

Published by Gray Publishing, Tunbridge Wells, Kent, on behalf of NCCHTA.

Printed on acid-free paper in the UK by St Edmundsbury Press Ltd, Bury St Edmunds, Suffolk. 


\title{
Abstract
}

\section{The clinical and cost-effectiveness of left ventricular assist devices for end-stage heart failure: a systematic review and economic evaluation}

\author{
AJ Clegg, I* DA Scott, ${ }^{2}$ E Loveman, ' J Colquitt, ' J Hutchinson, ${ }^{2}$ P Royle ${ }^{3}$ \\ and J Bryant ${ }^{\prime}$ \\ I Southampton Health Technology Assessments Centre, Wessex Institute for Health Research and Development, \\ University of Southampton, UK \\ ${ }^{2}$ Fourth Hurdle Consulting Ltd, London, UK \\ ${ }^{3}$ University of Aberdeen, UK \\ * Corresponding author
}

Objectives: To assess the clinical and cost-effectiveness of left ventricular assist devices (LVADs) as a bridge to heart transplantation (BTT), as a bridge to myocardial recovery (BTR) or as a long-term chronic support (LTCS) for people with end-stage heart failure (ESHF). Data sources: For the systematic review, electronic databases and bibliographies of related publications plus experts and manufacturers. For the economic evaluations, data originated from the systematic review of clinical and cost-effectiveness, UK hospitals, device manufacturers and expert opinion.

Review methods: For the systematic review, studies were selected and assessed against a set of rigorous criteria; data were then synthesised using a narrative approach through subgroup analysis based on the indication for treatment, type of LVAD and quality of studies. The economic evaluation developed two models to evaluate the use of LVADs, first as a BTT and second as LTCS for patients suffering from ESHF. Results: Sixteen studies assessed the clinical effectiveness of LVADs as a BTT. Despite the poor methodological quality of the evidence, LVADs appeared beneficial compared to other treatment options (i.e. inotropic agents or usual care) or to no care (i.e. the natural history of ESHF) improving the survival of people with ESHF during the period of support and following heart transplantation. Patients supported by an LVAD appeared to have an improved functional status compared with those on usual care and experienced an improvement in their quality of life from before device implantation to the period during support. Serious adverse events are a risk for patients with an LVAD. With a scarcity of evidence directly comparing different devices, it is difficult to identify specific devices as the most clinically effective. The HeartMate LVAD is the only device that has evidence comparing it with the different alternatives, appearing to be more clinically effective than inotropic agents and usual care and as clinically effective as the Novacor device. Second generation devices, such as Jarvik 2000 and MicroMed Debakey LVADs, are early in their development but show considerable promise that should be assessed through long-term studies. Evidence of the clinical effectiveness of LVADs as a BTR was limited to seven non-comparative observational studies that appeared to show that the LVADs were beneficial in providing support until myocardial recovery. It was not possible to assess whether the LVADs are more effective than other alternatives or specific devices. No evidence was found on the quality of life or functional status of patients and limited information on adverse events was reported. Six studies assessed the clinical effectiveness of LVADs as an LTCS and from these it was evident that LVADs provided benefits in terms of improved survival, functional status and quality of life. Nineteen studies assessed the costs and costeffectiveness of LVADS for people with ESHF, with the majority being simple costing studies and very few studies of the cost-effectiveness of LVADs. With no relevant cost-effectiveness studies available, an economic evaluation for BTT and LTCS was developed. The economic evaluation has shown that neither LVAD indication considered, that is, BTT and LTCS, is a costeffective use. For the HeartMate LVAD used as a BTT the cost per QALY was $£ 65,242$. In the less restrictive indication, LTCS, where LVADs are not just given to patients awaiting transplantation, the analysis has shown that LTCS is not cost-effective. The baseline 
cost per QALY of the first-generation HeartMate LVAD was $E \mid 70,616$. One- and multi-way sensitivity analysis had limited effect on the cost per QALY. A hypothetical scenario based on the cost of a second-generation MicroMed DeBakey device illustrated that a $60 \%$ improvement in survival over first-generation devices was necessary before the incremental costeffectiveness approached $€ 40,000$ per QALY.

Conclusions: Although the review showed that LVADs are clinically effective as a BTT with ESHF, the economic evaluation indicated that they are not cost-effective.
With the limited and declining availability of donor hearts for transplantation, it appears that the future of the technology is in its use as an LTCS. Further research is needed to examine the clinical effectiveness of LVADs for people with ESHF, assessing patient survival, functional ability, quality of life and adverse events. Evaluations of the clinical effectiveness of LVADs should include economic evaluations, as well as data on quality of life, utilities, resources and costs. A systematic review of the epidemiology of ESHF should be undertaken to assess its potential impact. 


\section{Contents}

List of abbreviations

\section{Executive summary}

I Aim and background

Aim

Background

Heart failure

Epidemiology of heart failure

Heart failure: aetiology and patient

characteristics

Prognosis of heart failure ..........................

Epidemiology of end-stage heart failure ...

End-stage heart failure and the needs and

demands for healthcare

Current service provision for heart

failure

Description of LVADs

Assessing the effectiveness of LVADs for

ESHF

2 Research methods for systematic review of clinical and cost-effectiveness of LVADs for ESHF

Research question

3 Systematic review of the clinical effectiveness of LVADs for people with ESHF .............. 27

Introduction

LVADs as a bridge to heart transplantation for ESHF

LVADs as a bridge to recovery

LVADs as a long-term chronic support ..... 58

4 Systematic review of the costs and cost-effectiveness of LVADs for people with ESHF

Introduction

Quantity of research

Methodological quality of research

LVADs as a bridge to heart transplantation for people with ESHF

LVADs as a bridge to recovery for people

with ESHF

LVADs as a long-term chronic support for people with ESHF

Discussion

Conclusions from the systematic review of costs and cost-effectiveness of LVADs for ESHF

vii

ix

1

1

1

2

4

8

8
8

\section{Conclusions}

Implications for practice ........................ 121

Recommendations for future research ....... 121

Acknowledgements

References

125

Appendix I Devices used as LVADs and expert advisory group assessment of the devices that should be included in the systematic review and economic evaluation of the clinical and cost effectiveness of LVADs for ESHF

Appendix 2 Original research protocol .... 135

Appendix 3 Sources of information, including databases searched and search terms 139

Appendix 4 List of manufacturers of LVADs included in the study and response to invitation to submit information

Appendix 5 List of studies meeting the general inclusion criteria for the systematic review of clinical effectiveness of LVADs for people with ESHF that were excluded from the assessment as they were judged to be too low on the hierarchy of evidence 
Appendix 6 List of a selection of studies excluded from the systematic review

Appendix 7 Data extraction form for systematic reviews

Appendix 8 Data extraction form for primary studies

Appendix 9 Data extraction and quality assessment of economic evaluations and costing studies

Appendix 10 Quality assessment scales for systematic reviews

Appendix I I Quality assessment for primary studies

Appendix 12 Summary of the evidence of clinical effectiveness of the HeartMate LVAD as a BTT for people with ESHF

Appendix I3 Summary of the evidence of clinical effectiveness of the Novacor LVAD as a BTT for people with ESHF

Appendix 14 Summary of the evidence of clinical effectiveness of the Toyobo LVAD as a BTT for people with ESHF

Appendix I5 Summary of the evidence of clinical effectiveness of the Thoratec LVAD as a BTT for people with ESHF

Appendix 16 Summary of the evidence of clinical effectiveness of the Jarvik 2000 LVAD as a BTT for people with ESHF

Appendix 17 Summary of the evidence of clinical effectiveness of the MicroMed DeBakey LVAD as a BTT for people with ESHF

Appendix 18 Summary of the evidence of clinical effectiveness of the Abiomed device as a BTR for people with ESHF

Appendix 19 Summary of the evidence of clinical effectiveness of the HeartMate LVAD as a BTR for people with ESHF

Appendix 20 Summary of the evidence of clinical effectiveness of the Novacor LVAD as a BTR for people with ESHF
Appendix 21 Summary of the evidence of clinical effectiveness of the Thoratec LVAD as a BTR for people with ESHF

Appendix 22 Summary of the evidence of clinical effectiveness of the Toyobo LVAD as a BTR for people with ESHF

Appendix 23 Summary of the evidence of clinical effectiveness of the HeartMate LVAD as an LTCS for people with ESHF .. 273

Appendix 24 Summary of the evidence of clinical effectiveness of the LionHeart LVAD as an LTCS for people with ESHF

Appendix 25 Summary of the evidence of clinical effectiveness of the Novacor LVAD as an LTCS for people with ESHF 285

Appendix 26 Summary of the evidence of clinical effectiveness of the Toyobo LVAD as an LTCS for people with ESHF 289

Appendix 27 Summary of the evidence of clinical effectiveness of the Jarvik 2000 LVADs as an LTCS for people with ESHF

Appendix 28 Data extraction forms economic/costing studies

Appendix 29 Data extraction forms economic/costing studies as abstracts only

Appendix 30 Data extraction forms utility studies

Appendix 31 EVAD costs/charges for Papworth Hospital (Fawell J, Papworth Hospital NHS Trust: personal communication, 2004)

Appendix 32 List of experts who agreed to act as advisors to the project, their stated conflicts of interest and contributions to the project

\section{Health Technology Assessment reports} published to date

Health Technology Assessment

Programme 


\section{List of abbreviations}

\begin{tabular}{|c|c|c|c|}
\hline ACE & angiotensin-converting enzyme & GVS & cytomegalovirus \\
\hline ANOVA & analysis of variance & DIC & $\begin{array}{l}\text { disseminated intravascular } \\
\text { coagulation }\end{array}$ \\
\hline AUC & area under the curve & ECG & electrocardiogram \\
\hline BiVAD & biventricular assist device & ECMO & $\begin{array}{l}\text { extracorporeal membrane } \\
\text { oxygenation }\end{array}$ \\
\hline BP & blood pressure & ESHF & end-stage heart failure \\
\hline BPM & beats per minute & EVAD & $\begin{array}{l}\text { Evaluation of the Ventricular } \\
\text { Assist Device study }\end{array}$ \\
\hline BSC & best supportive care & FDA & Food and Drug Administration \\
\hline BTR & bridge to recovery & IABP & intra-aortic balloon pump \\
\hline BTT & bridge to heart transplantation & ICER & incremental cost-effectiveness ratio \\
\hline BUN & blood urea nitrogen & $\mathrm{ICU}$ & intensive care unit \\
\hline $\mathrm{CAB}$ & coronary artery bypass & INR & international normalised ratio \\
\hline CAD & coronary artery disease & IP & implanted pneumatic \\
\hline CCT & controlled clinical trial & ISHLT & $\begin{array}{l}\text { International Society for Heart } \\
\text { and Lung Transplantation }\end{array}$ \\
\hline CE & CE marking & ITU & intensive treatment unit \\
\hline CEAC & $\begin{array}{l}\text { cost-effectiveness acceptability } \\
\text { curve }\end{array}$ & i.v. & intravenous \\
\hline CETS & $\begin{array}{l}\text { Conseil d'Évaluation des } \\
\text { Technologies de la Santé du } \\
\text { Québec }\end{array}$ & $\begin{array}{l}\text { IVAD } \\
\text { LOS } \\
\text { LTCS }\end{array}$ & $\begin{array}{l}\text { implantable ventricular assist device } \\
\text { length of stay } \\
\text { long-term chronic support }\end{array}$ \\
\hline $\mathrm{CHF}$ & chronic heart failure & LVAD & left ventricular assist device \\
\hline CI & confidence interval & LVAS & left ventricular assist system \\
\hline CMV & cytomegalovirus & LVEF & left ventricular ejection fraction \\
\hline CPB & coronary pulmonary bypass & LVIDd & $\begin{array}{l}\text { left ventricular end-diastolic } \\
\text { diameter }\end{array}$ \\
\hline CRD & $\begin{array}{l}\text { Centre for Reviews and } \\
\text { Dissemination }\end{array}$ & & continued \\
\hline
\end{tabular}




\section{List of abbreviations continued}

\begin{tabular}{|c|c|c|c|}
\hline LYG & life-year gained & $\mathrm{RR}$ & relative risk \\
\hline MI & myocardial infarction & RVAD & right ventricular assist device \\
\hline MLHFQ & $\begin{array}{l}\text { Minnesota Living with Heart } \\
\text { Failure Questionnaire }\end{array}$ & RVEF & right ventricular ejection fraction \\
\hline MOF & multiple organ failure & $\mathrm{SD}$ & standard deviation \\
\hline NICE & National Institute for Health and & $\mathrm{SE}$ & standard error \\
\hline & Clinical Excellence & SEM & standard error of the mean \\
\hline ns & not significant & SF-36 & Short Form with 36 Items \\
\hline NSCAG & $\begin{array}{l}\text { National Specialist Commissioning } \\
\text { Advisory Group }\end{array}$ & SG & standard gamble \\
\hline NYHA & New York Heart Association & TAH & total artificial heart \\
\hline OR & odds ratio & TTO & time trade-off \\
\hline PATT & $\begin{array}{l}\text { permanent alternative to } \\
\text { transplant }\end{array}$ & UNOS & $\begin{array}{l}\text { United Network for Organ } \\
\text { Sharing }\end{array}$ \\
\hline PT & post-transplantation & VAD & ventricular assist device \\
\hline QALY & quality-adjusted life-year & VAS & visual analogue scale \\
\hline QoL & quality of life & $\mathrm{VE}$ & vented electric \\
\hline Q-TWIST & $\begin{array}{l}\text { quality-adjusted time without } \\
\text { symptoms and toxicity }\end{array}$ & $\mathrm{VF}$ & ventricular fibrillation \\
\hline RCT & randomised controlled trial. & VT & ventricular tachycardia \\
\hline REMATCH & $\begin{array}{l}\text { Randomized Evaluation of } \\
\text { Mechanical Assistance in } \\
\text { Treatment of Chronic Heart Failure }\end{array}$ & & \\
\hline
\end{tabular}

All abbreviations that have been used in this report are listed here unless the abbreviation is well known (e.g. NHS), or it has been used only once, or it is a non-standard abbreviation used only in figures/tables/appendices in which case the abbreviation is defined in the figure legend or at the end of the table. 


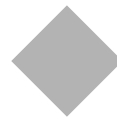 \\ Executive summary}

\section{Background}

Heart failure is an increasing health problem in England and Wales. Its incidence and prevalence are increasing, leading to frequent admissions to hospital and long-term drug costs. Concerns about the effects on the duration and quality of life of people and costs upon the NHS have resulted in several government policy initiatives. End-stage heart failure (ESHF) is associated with major disability and a poor prognosis. Heart transplantation has become the accepted form of treatment for people with ESHF, improving survival and patient quality of life. With continued decreases in organ donation in England and Wales, it is an option available to few. Mechanical circulatory support through left ventricular assist devices (LVADs) has attracted increased interest as an option for patients with ESHF whether as a bridge to heart transplantation (BTT), as a bridge to myocardial recovery (BTR) or as a long-term chronic support (LTCS). Continued developments of LVADs, declining costs and improvements in associated care have made their wider use a reality.

\section{Objectives}

The objectives of this study were to carry out a systematic review and an economic evaluation to examine the clinical and cost-effectiveness of LVADS as a BTT, BTR and LTCS for people with ESHF. The study focused on the effect of LVADs on the duration and quality of life of people with ESHF, the groups who may benefit from their use and assesses the implications for developing such a service within the NHS in England and Wales.

\section{Methods}

A systematic review of the evidence and an economic evaluation were undertaken using $a$ priori methods.

\section{Data sources}

Eighteen electronic databases were searched from inception to October 2003. Bibliographies of related papers were assessed for relevant studies and experts and manufacturers were contacted to identify additional published and unpublished references.

\section{Study selection}

Studies were included if they fulfilled the following criteria:

- Interventions: studies that evaluated currently available LVADs used as a BTT, BTR and LTCS for people with ESHF were considered for inclusion.

- Participants: people aged 16 years or older with ESHF and considered suitable for receipt of an LVAD as BTT, BTR or LTCS.

- Outcomes: survival, functional capacity [e.g. New York Heart Association (NYHA) functional classification, activities of daily living] and quality of life were the primary outcome measures considered within the systematic review.

- Design: systematic reviews, randomised controlled trials (RCTs), controlled clinical trials, cohort studies, case series, case studies, economic evaluations and cost studies were included.

Studies identified were assessed for inclusion through two stages with titles and abstracts and full papers of retrieved studies assessed by two reviewers, with differences in decisions resolved through discussion or through recourse to a third, independent reviewer.

\section{Data extraction and quality assessment}

Data were extracted by two reviewers using data extraction forms developed a priori, with any disagreements resolved through discussion or through recourse to independent assessment by a third reviewer. The methodological quality of the studies included in the systematic review of clinical and cost-effectiveness were assessed using recognised quality assessment tools using individual components of methodological quality rather than relying upon summary scores. The quality criteria used were applied by two reviewers, with any disagreements resolved through discussion or through recourse to a third, independent reviewer. 


\section{Data synthesis}

Studies were synthesised using a narrative approach through subgroup analysis based on the indication for treatment, type of LVAD and quality of studies.

\section{Economic evaluation}

The economic evaluation developed two models to evaluate the use of LVADs, first as a BTT and second as LTCS for patients suffering from ESHF. Insufficient data prevented the assessment of the use of LVADS as a BTR. The outcomes in the evaluation were assessed in terms of the benefits to patients (survival and quality of life) and costs incurred, with results presented in terms of a cost-utility analysis. Although medical therapy was the comparator treatment in the two models, the patients differed. Patients in the BTT model were those on the heart transplantation waiting list, whereas those in the LTCS model were excluded from the heart transplant waiting list owing to the severity of their condition. The models focused on those LVADs that were found in the systematic review to be clinically effective for the different indications and relevant to the UK setting.

\section{Results}

\section{Clinical effectiveness of LVADs as a BTT for people with ESHF}

Sixteen studies (one controlled clinical trial, five cohort studies with comparators, five cohort studies with no comparator, three case series and two case reports) assessed the clinical effectiveness of LVADs as a BTT, 11 studies of first-generation devices and five of second-generation devices. The methodological quality of the studies was generally weak, reflecting the quasi-experimental and observational study designs used and poor reporting of study characteristics. Despite the poor quality evidence, LVADs compared with other treatment options appeared to be beneficial for patients with ESHF when assessed using patient survival, functional status and quality of life. When compared with inotropic agents, LVADs appeared to provide a benefit in patient survival that increased with the length of support (difference in actuarial survival: 1 month 3\%; 3 months 17\%) and extended beyond heart transplantation (difference in actuarial survival: 1 year 24\%; 4 years 30\%). Comparisons of the use of LVADs with usual care were less certain, with outcomes varying from no difference in survival to, or after, heart transplantation, to improved survival for LVADs patients to heart transplantation (survival difference: range 14-59\%) and post-transplantation (difference in actuarial survival: 1 and 2 years $100 \%$ ). Studies of LVADs which did not include a comparator were often the only evidence available, particularly for the new devices. In these studies, implantation of an LVAD provided support for up to 390 days, with as many as $70 \%$ of patients surviving to transplantation. Comparisons of different LVADs were limited. Only the HeartMate and Novacor LVADs were compared, with little difference in survival to transplantation. There was limited evidence assessing the effects of LVADs on the functional status and quality of life of patients with ESHF BTT. Patients supported by an LVAD appeared to have an improved functional status compared with those on usual care. Also, patients with an LVAD experienced an improvement in their quality of life from before implantation of the device to the period during support. The use of LVADs is associated with risks of adverse events, with patients suffering mechanical device failures, bleeding, thromboembolic events, infections, reoperations and psychiatric conditions. Adverse events rates varied between different LVADs and studies and some caution should be exercised in interpreting these results. With a scarcity of evidence directly comparing the different LVADs it is difficult, and perhaps inappropriate, to identify specific devices as the most clinically effective. However, the HeartMate LVAD is the only device that has evidence comparing it with several different alternatives, appearing to be more clinically effective than inotropic agents and usual care and as clinically effective as the Novacor device. Of the second-generation devices, the evidence suggests limited difference in the clinical effectiveness of the Jarvik 2000 and the MicroMed DeBakey LVADs. Although early in their development, these second-generation devices appear to show lower rates of adverse events, such as infection, bleeding and thromboembolism, which have affected the development and use of the first-generation devices. However, longer term outcomes are needed to ensure that these apparent benefits are maintained in practice and that the consequences associated with the nonpulsatile nature of the devices do not result in additional adverse events.

\section{Clinical effectiveness of LVADs as a BTR for people with ESHF}

Evidence of the clinical effectiveness of LVADs as a BTR for people with ESHF was limited to seven non-comparative observational studies (two case series and five case reports) of first-generation devices, which were judged to be of poor methodological quality. The seven identified studies appeared to show that the LVADs provided 
benefit in providing support for the patients until myocardial recovery. As there were no direct comparisons of different interventions, it is not possible to assess whether the LVADs are more effective than other alternatives or specific devices. No evidence was found to judge the effects of the devices on the quality of life or functional status of patients. Limited information on adverse events was reported, although infections and bleeding were the main concerns.

\section{Clinical effectiveness of LVADs as an LTCS for people with ESHF}

Six studies (one RCT, one case series and four case reports) assessed the clinical effectiveness of LVADs as an LTCS for people with ESHF. Although the nature and methodological quality of the evidence varied between the different devices, it was evident that LVADs provided benefits for patients in terms of improved survival, functional status and quality of life. The REMATCH trial provided good-quality evidence that the HeartMate LVAD provided a statistically significant $48 \%$ reduction in the risk of death from any cause when compared with optimal medical management. Actuarial survival was significantly higher for patients with the HeartMate LVAD compared with optimal medical management at 1 year (52\% versus $25 \%)$ and 2 years (23\% versus $8 \%$ ) follow-up. Importantly, improvements in 1-year survival were evident for patients aged under 60 years and those aged 60-69 years. Less rigorous evidence for the Novacor, Toyobo and Jarvik 2000 devices showed relatively high survival (90\%), with patients supported for up to 4 years. Limited information on changes in patients' quality of life and functional status suggested that patients experienced improvements on specific scales following implantation of the HeartMate and Jarvik LVADs. Inevitably there are adverse events associated with the use of LVADs, with device malfunctions, infection and bleeding associated with their use. The HeartMate LVAD was associated with twice as many serious adverse events than optimal medical management, with significantly higher rates of non-neurological bleeding and neurological dysfunction. Other adverse events affected the different treatment options, whether devices or drug treatment. Despite these adverse events, the benefits of these LVADs appear to outweigh limitations. Evidence of the clinical effectiveness of the different devices indicates that the HeartMate LVAD appears to be effective when compared with optimal medical management. For the second-generation devices, the early evidence suggests that the Jarvik 2000 shows promise; however, further research is needed to assess whether these benefits are replicated in the longer term and whether there will be any long-term consequences associated with the change in the nature of the circulatory support.

\section{Systematic review of the cost- effectiveness of LVADs for people with ESHF}

Nineteen studies assessed the costs and costeffectiveness of LVADS for people with ESHF, with the majority being simple costing studies and very few studies of the cost-effectiveness of LVADs. A number of the costing studies had serious methodological flaws. Even those judged of 'higher quality' had caveats limiting applicability and generalisability to a UK population.

Significant limitations were the limited sample size and the lack of comparators against which to judge the significance of the reported costs. There was only one UK-based cost-utility analysis, which was populated with costs based on treatment protocols, data from individual NHS Trust finance departments and utilities derived from a US base study. The UK study reported cost per qualityadjusted life-year (QALY) values at the boundary of acceptability given recent decision-making. Based on total treatment costs of both LVAD BTT support and heart transplantation, the discounted cost per QALY was approximately $£ 39,790$ (range $£ 28,510-74,000)$. Threshold analysis found that an LVAD device and procedure cost of $£ 19,300$ would equate to a cost per QALY of $£ 20,000$ or less.

\section{Economic evaluation to assess the cost-effectiveness of LVADs for people with ESHF within the UK}

The economic evaluation has shown that neither LVAD indication considered, that is, BTT and LTCS, is a cost-effective use. For the HeartMate LVAD used as a BTT the cost per QALY was $£ 65,242$. Stochastic simulation calculated the $95 \%$ confidence interval at $£ 34,194$ to $£ 364,564$. Only when the survival gain falls significantly does the cost-effectiveness rise to such upper bounds. A cost-effectiveness acceptability curve showed the likelihood of acceptability at current costeffectiveness thresholds. The BTT indication approaches cost-effectiveness only when the oneoff costs associated with an LVAD fall considerably. At a combined LVAD device and operation cost of $£ 50,000$ (compared with $£ 87,877$ in our model), the cost per QALY fell to approximately $£ 40,000$. Unfortunately, even assuming this eventuality, the BTT indication use parallels an ever-decreasing supply of donor hearts capping the ability of this innovative technology to yield widespread benefit. 
In the less restrictive indication, LTCS, where LVADs are not just given to patients awaiting transplantation, the analysis has shown that LTCS is not cost-effective. The baseline cost per QALY of the first-generation HeartMate LVAD was $£ 170,616$. One- and multi-way sensitivity analysis had limited effect on the cost per QALY. A hypothetical scenario based on the cost of a second-generation MicroMed DeBakey device illustrated that a $60 \%$ improvement in survival over first-generation devices was necessary before the incremental cost-effectiveness approached $£ 40,000$ per QALY. Although the analyses recognise the benefits in terms of survival and quality of life, these are outweighed by associated increases in cost. Uncertainties remain, particularly with the lack of trial data on LTCS, research on second-generation devices and ongoing costs of medical management. The use of expert-based utilities in the LTCS data is also innovative but the sensitivity testing has shown this not to be an influential factor on cost-effectiveness at the margin.

\section{Discussion and conclusions}

\section{Implications for practice}

Although the systematic review of clinical effectiveness showed that LVADs are clinically effective as a BTT for people with ESHF, the economic evaluation indicated that they are not cost-effective. With the limited and declining availability of donor hearts for transplantation, it appears that the future of the technology is in its use as an LTCS. At present the evidence of clinical and cost-effectiveness of LVADs as an LTCS is less certain, particularly for the second-generation devices, and further study is needed. The limited research available showed some clinical benefit for patients receiving LVADs as an LTCS, but the economic evaluation suggested that they were not a cost-effective option. Limited numbers of patients with ESHF receive an LVAD within England and Wales and uncertainty remains about the potential need and demand that may exist. As a consequence, it is likely that there will be limited availability of clinical teams who can undertake these procedures and manage the patients subsequently and there would need to be a rapid increase in the training of staff and a step change in the development of the necessary infrastructure. To provide the service to meet the needs of a conservative estimate of 3000 patients would result in a discounted cost to the NHS annually of $£ 321$ million. With less conservative estimates putting the potential need at between 7000 and 34,000 patients, the actual cost may be far higher.

\section{Recommendations for future research}

Although LVADs appear to be effective in improving the survival of patients with ESHF, whether as a BTT, BTR or LTCS, the methodological quality and strength of the evidence are poor. Further research is needed to examine the clinical effectiveness of LVADs for people with ESHF, assessing patient survival, functional ability, quality of life and adverse events. Although difficult to undertake with such fast-changing technologies, such evaluations should be RCTs and look at the head-to-head comparisons of different devices or usual medical care. Importantly, these studies should encompass the breadth of patient groups that may benefit from these devices. Evaluations of the clinical effectiveness of LVADs should include economic evaluations. It has become evident from this study that data for undertaking such studies are very limited. Data on quality of life, utilities, resources and costs are not readily available. Also, there is limited research on the epidemiology of ESHF and, as a consequence, it is difficult to establish the possible need and demand for the service. A systematic review of the epidemiology of ESHF should be undertaken to assess its potential impact. 


\section{Chapter I}

\section{Aim and background}

\begin{abstract}
Aim
To review systematically the evidence assessing the clinical and cost-effectiveness of left ventricular assist devices (LVADs) as a bridge to heart transplantation (BTT), bridge to recovery (BTR) and as a long-term chronic support (LTCS) for people with end-stage heart failure (ESHF). It will examine the effect of LVADs on the duration and quality of life (QoL) of people with ESHF, in addition to providing the opportunity to assess the number of people who could benefit from the use of LVADs, the possible demand that may be placed upon the NHS in England and Wales and the costs of operating such a service. In addition, the study will identify the different groups who may benefit from the use of LVADs. If the systematic review of economic evaluations shows that there are no appropriate good-quality economic evaluations, an economic model relevant to the UK setting will be developed.
\end{abstract}

\section{Background}

Heart failure is a major health problem in England and Wales. Its incidence and prevalence continue to increase, partly owing to an ageing population but also to a high prevalence of cardiovascular disease. ${ }^{1}$ Heart failure is associated with substantial morbidity and mortality leading to frequent admissions to hospital and long-term drug costs. As a consequence, it is a major cost to the NHS and increasingly the focus of policy initiatives. ${ }^{2,3}$

Heart failure in England and Wales is usually due to ischaemic heart disease, but is also caused by cardiomyopathy (often in young people), hypertension and myocarditis. It has a wide clinical spectrum from mild heart failure that has little effect on a person's day-to-day life, through to severe or ESHF, which is associated with major disability and a poor prognosis (1-year mortality of $50 \%$ or more). ${ }^{1,4,5}$ This is despite advances in drug treatment which have improved survival, such as angiotensin-converting enzyme (ACE) inhibitors and beta-blockers. ${ }^{6}$

Heart transplantation has become an accepted form of surgical treatment for patients at risk of dying from, or incapacitated by, severe or ESHF. ${ }^{7}$ Allograft transplantation has resulted in actuarial survival of $81 \%$ at 1 year, $65 \%$ at 5 years and $45 \%$ at 10 years ${ }^{8}$ with return to a near normal QoL. ${ }^{9}$ Scarcity of donor organs means that only about 150 heart transplants are performed annually in the UK, and that fewer than half of potential recipients receive an organ. ${ }^{9}$ Evidence suggests the potential demand in patients under 60 years old for transplantation in England and Wales is between 10 and 15 per million, far exceeding the supply of donor hearts ( 2 per million). ${ }^{10}$ The supply of donor organs has decreased considerably in the UK over the last 10 years, which, along with improvements in the treatment of heart failure, has led to a decrease in the number of people admitted to the transplant waiting list and receiving a heart transplant (Table 1). The growing disparity between the supply of donor organs and the need and demand for heart transplantation has resulted in prolonged time and increasing mortality on the waiting list. A large number of potential transplant candidates, estimated at between 20 and $40 \%$, die whilst on the waiting list before a donor heart becomes available. ${ }^{11}$ As a partial consequence, mechanical circulatory support has increasingly been considered an option for patients with severe heart failure.

LVADs are mechanical pumps that are used to help people with ESHF who suffer from considerable limitations and discomfort from any physical activity owing to cardiac disease, despite the use of maximal medical therapy. The devices assist the function of the damaged left ventricle and help to restore normal haemodynamics and end-organ blood flow. ${ }^{12}$ Originally developed during the 1960s in the USA as a BTT, they have increasingly been thought useful as a BTR, with the potential for being an LTCS or permanent alternative to heart transplantation. ${ }^{12}$ As a BTT, LVADs provide patients who have ESHF without irreversible end-organ failure with an increased chance of survival while awaiting a donor heart, and also an increased QoL and improved physiological status at transplantation. ${ }^{12,13}$ Duration of support to heart transplantation has extended to nearly 2 years for some people, although most are supported for a shorter period. Prolonged mechanical LVAD support in the BTT 
TABLE I Annual figures for people registered on the heart-only transplant list, the number who died on the heart-only transplant list, the number of heart-only transplants and the number of donors of hearts and heart/lung blocks in the UK, 1992-2003

\begin{tabular}{|c|c|c|c|c|c|c|c|}
\hline \multirow[t]{2}{*}{ Year } & \multicolumn{2}{|c|}{ Transplant list } & \multicolumn{2}{|c|}{ Died } & \multirow[t]{2}{*}{ Transplants } & \multicolumn{2}{|c|}{ Donors } \\
\hline & Active & Suspended $^{a}$ & Active & Suspended $^{a}$ & & Heart only & Heart/lung \\
\hline 1992 & 255 & 46 & 78 & 1 & 322 & 278 & 106 \\
\hline 1993 & 237 & 28 & 69 & 2 & 302 & 254 & 95 \\
\hline 1994 & 253 & 41 & 67 & 1 & 311 & 273 & $13 \mid$ \\
\hline 1995 & 277 & 44 & 60 & 2 & 319 & 246 & 128 \\
\hline 1996 & 241 & 62 & 80 & 5 & 276 & 223 & 125 \\
\hline 1997 & 230 & 75 & 67 & 2 & 264 & 201 & 104 \\
\hline 1998 & 232 & 43 & 58 & 7 & 261 & 206 & 108 \\
\hline 1999 & 212 & 52 & 59 & 3 & 227 & 159 & 115 \\
\hline 2000 & 134 & 22 & 41 & I & 204 & 143 & 81 \\
\hline 2001 & 105 & 8 & 32 & 2 & 166 & 142 & 69 \\
\hline 2002 & 96 & 3 & 12 & 2 & 158 & III & 57 \\
\hline 2003 & 99 & 5 & 8 & 1 & 148 & 94 & 74 \\
\hline
\end{tabular}

setting has led to the observation that in some patients the myocardium shows the propensity to recover, allowing removal of the device rather than the previously necessary heart transplantation, termed BTR. ${ }^{12,14}$ The duration of LVAD support for those who recover varies from days to up to 1 year. However, usually patients classified as a BTR have received an LVAD as a BTT, but recover from the underlying condition before heart transplantation is possible. The increasing duration of LVAD implantation in many patients has led to the question of the suitability of implantable LVADs for permanent use as an alternative to heart transplantation, often refered to as LTCS or destination therapy. ${ }^{12}$ This may have the advantage of earlier intervention and rehabilitation of patients with ESHF, and avoids the not inconsiderable risks associated with immunosuppression and organ rejection following heart transplantation. It may also become possible to treat those patients who, owing to age or disease severity, would not have been considered eligible for heart transplantation in the first place. ${ }^{15}$ The use of LVADs as an LTCS is relatively new and uncertainty remains as to the duration of support possible. However, a patient with a Jarvik 2000 LVAD has survived for over 4 years and remains alive. The continued development of LVADs (e.g. miniaturisation, low power use and ease of implantation of axial flow rotary pumps) and the declining cost and the improvements in associated care (e.g. cyclosporin immunosuppression) have made their wider use, particularly as a permanent replacement, a reality. ${ }^{10}$
This chapter examines the extent of the problem caused by heart failure, particularly ESHF, in England and Wales. It looks to describe the condition and its epidemiology, aetiology and prognosis prior to estimating the possible need and demand for healthcare from those with ESHF. The chapter briefly outlines the current service provided within the NHS in England and Wales to ESHF patients prior to outlining the interventions considered within this study.

\section{Heart failure}

\section{Definition}

Heart failure is a complex clinical syndrome encompassing several different clinical states (Table 2). Defining and describing heart failure can be difficult owing to the lack of agreed and standardised criteria, with terminology differing widely. Commonly it is defined as a disease characterised by a decline in the heart's ability to pump blood around a person's body at normal filling pressures to meet its metabolic needs. ${ }^{16,17} \mathrm{It}$ can result from any structural or functional cardiac disorder that affects the ability of the ventricle to fill with or eject blood. ${ }^{18}$ Historically it has been described through different features such as duration, initiating mechanism, the ventricle primarily affected, the clinical syndrome and the pathophysiological mechanisms, including 'acute or chronic', 'left or right', 'forward or backward', 'congestive or non-congestive', 'high or low output' and 'systolic, diastolic or mixed' heart failure. ${ }^{19}$ For the purposes of assessment and management, the 
TABLE 2 International Classification of Diseases Version $10^{20}$

\begin{tabular}{|c|c|c|}
\hline Code & \multicolumn{2}{|l|}{ Disease } \\
\hline 150 & \multicolumn{2}{|c|}{$\begin{array}{l}\text { - abortion or ectopic or molar pregnancy }(\mathrm{O} 00-\mathrm{O} 07, \mathrm{O} 08.8) \\
\text { - obstetric surgery and procedures (O75.4) } \\
\text { - due to hypertension (III.0) } \\
\text { - with renal disease (II3.-) } \\
\text { - following cardiac surgery of due to presence of cardiac prosthesis (197.I) } \\
\text { - neonatal cardiac failure (P29.0) }\end{array}$} \\
\hline 150.0 & \multicolumn{2}{|c|}{$\begin{array}{l}\text { Congestive heart failure } \\
\text { Congestive heart disease } \\
\text { Right ventricular failure (secondary to left heart failure) }\end{array}$} \\
\hline I50.I & $\begin{array}{l}\text { Left ventricular failure } \\
\text { Acute oedema of lung } \\
\text { Acute pulmonary oedema } \\
\text { Cardiac asthma } \\
\text { Left heart failure }\end{array}$ & $\begin{array}{l}\text { With mention of heart disease } \\
\text { NOS or heart failure }\end{array}$ \\
\hline 150.9 & $\begin{array}{l}\text { Heart failure, unspecified } \\
\text { Biventricular failure } \\
\text { Cardiac, heart or myocardi }\end{array}$ & \\
\hline
\end{tabular}

distinction between acute and chronic heart failure $(\mathrm{CHF})$ is usually made. Acute heart failure has a dramatic clinical onset with an acutely dyspnoeic patient (extreme difficulty in breathing) demonstrating visible signs of cardiovascular insufficiency (inadequate operation of cardiovascular system) such as tachycardia (fast heart beat), pulmonary or peripheral oedema (fluid retention in the lungs and extremities of the body) and under perfusion of systemic organs (inadequate blood flow through the body). Chronic heart failure is a more subtle condition, which may have a more gradual onset. It is characterised by the onset and persistence of left ventricular dysfunction and pathophysiological changes to other related organs that produce symptoms and limit exercise. Often people will develop chronic heart failure and suffer acute exacerbations.

When heart failure occurs, the heart usually tries to compensate through changes in systemic vascular resistance and redistribution of blood flow (changes in blood pressure and flow). ${ }^{17}$ Although the heart may be capable of maintaining cardiac output at rest through such changes, it is unlikely to be able to cope with the increased demands that may result from normal exercise. Despite the heart's attempts to adapt, the demands and stresses on the cardiovascular and related systems, such as the renal and hepatic systems, tend to lead to further decline and, without effective treatment, to severe heart failure (decompensated or ESHF) and eventual death.

Although heart failure encompasses several different clinical conditions, it does have a wellrecognised clinical pattern. As already indicated, the key features of heart failure are that it is initiated by a reduction in effective cardiovascular (usually left ventricular) functional reserve, linked with symptoms either at rest or at an unexpectedly low level of exertion and associated with characteristic pathophysiological changes (including biochemical, hormonal, metabolic or functional) in many disparate organ systems. ${ }^{19}$ Generally, it may manifest itself clinically through patients suffering breathlessness (dyspnoea), weakness or fatigue or through fluid retention in tissues (peripheral oedema).

As a clinical syndrome, heart failure has many different causes. The main underlying cause of heart failure in the UK and most other western countries is coronary heart disease, which accounts for approximately two-thirds of cases. ${ }^{2,18,21}$ The remaining cases may have a non-ischaemic cause of heart failure such as hypertension, valvular disease, myocardial toxins or myocarditis, or may have no identifiable cause such as idiopathic dilated cardiomyopathy. ${ }^{2,18}$ 
TABLE 3 NYHA classification of heart failure 22,23

\begin{abstract}
Class I No limitation on activities: suffers from no symptoms from (performance of) ordinary activities. Patients with cardiac disease but without resulting limitations on physical activities. Ordinary physical activity does not cause fatigue, palpitation, dyspnoea or anginal pain

Class II Slight limitation on physical activities: comfortable at rest or mild exertion. Patients with cardiac disease resulting in slight limitation on physical activities. They are comfortable at rest. Ordinary physical activity results in fatigue, palpitation, dyspnoea or anginal pain

Class III Marked limitation on physical activities: comfortable only at rest. Patients with cardiac disease resulting in marked limitations on physical activities. They are comfortable at rest. Less than ordinary physical activity causes fatigue, palpitation, dyspnoea or anginal pain

Class IV Discomfort with any physical activity; should be completely rested or confined to bed. Patients with cardiac disease resulting in an inability to carry out any physical activity without discomfort. Symptoms of cardiac insufficiency or of the anginal syndrome may be present even at rest. If any physical activity is undertaken, discomfort is increased
\end{abstract}

Given the complexity of the syndrome and the differing underlying causes and subsequent treatment options, a range of disease classifications have been developed to assess the severity and impact of heart failure and its treatment on patients' lives. ${ }^{22}$ Most consider aspects of QoL, although they are often limited to measuring symptoms, functioning or depression. A widely used measure is the New York Heart Association (NYHA) functional classification of heart failure (Table 3). The classification relies on the assessment by the clinician of the occurrence of symptoms of heart failure that result from physical exercise, with the patient graded from I to IV on their degree of impairment. Patients in NYHA Class I have cardiac disease but are not limited in the activities they can undertake and do not suffer from the usual symptoms of fatigue, palpitation or anginal pain following ordinary exercise. Slight limitations are placed on NYHA Class II patients due to their cardiac condition. Although comfortable at rest or with mild physical exertion, ordinary physical exercise will result in the characteristic symptoms. Patients who deteriorate to NYHA Class III are unable to undertake any physical exercise without suffering fatigue, palpitation, dyspnoea or anginal pain. With increased severity of heart failure to NYHA Class IV, patients experience discomfort with any physical activity and will require complete rest or confinement to bed. Symptoms of cardiac insufficiency or of the anginal syndrome affect patients at rest and are accentuated with physical effort. Patients in NYHA Class IV are classified as suffering from ESHF and are candidates for heart transplantation or mechanical support.

Other classifications have been developed to take account of the evolution and progression of heart the American Heart Association Task Force on Practice Guidelines developed a four-stage classification of heart disease that should be used in conjunction with the NYHA classification (Table 4). ${ }^{18}$ In stage A, patients are at high risk for developing heart failure but have no structural disorder of the heart. These patients have identified risk factors of the disease, such as a familial or personal history of predisposing risk factors (e.g. alcohol abuse) or other diseases (e.g. rheumatic fever or cardiomyopathy). Patients in stage B have developed structural heart diseases that are strongly associated with heart failure, although they have not shown any signs or symptoms [e.g. previous myocardial infarction (MI)]. Those with current or prior symptoms of heart failure associated with underlying structural heart disease are classified as being in stage $\mathrm{C}$. For example, these patients may suffer from breathlessness or fatigue following left ventricular systolic dysfunction or are undergoing treatment for prior symptoms of heart failure. In stage $\mathrm{D}$, patients have advanced structural heart disease with recognised symptoms of heart failure at rest despite maximal medical therapy. These patients are suffering from refractory ESHF and require specialised interventions such as heart transplantation, circulatory assist device support, continuous intravenous support for symptom relief or hospice care.

\section{Epidemiology of heart failure}

Despite the increasing attention to heart failure, particularly in Europe and the USA, difficulties persist in developing an understanding of its epidemiology. ${ }^{23}$ Differences in the definition of heart failure and problems in identifying clear diagnostic criteria, and also difficulties in devising acceptable research criteria, have meant that 
TABLE 4 Stages of heart failure ${ }^{18}$

\begin{tabular}{|c|c|c|}
\hline Stage & Description & Examples \\
\hline A & $\begin{array}{l}\text { Patients at high risk of developing heart failure } \\
\text { because of the presence of conditions that are } \\
\text { strongly associated with the development of heart } \\
\text { failure. Such patients have no identified structural } \\
\text { or functional abnormalities of the pericardium, } \\
\text { myocardium or cardiac valves and have never } \\
\text { shown signs or symptoms of heart failure }\end{array}$ & $\begin{array}{l}\text { Systemic hypertension; coronary artery disease; } \\
\text { diabetes mellitus; history of cardiotoxic drug therapy or } \\
\text { alcohol abuse; personal history of rheumatic fever; } \\
\text { family history of rheumatic fever; family history of } \\
\text { cardiomyopathy }\end{array}$ \\
\hline B & $\begin{array}{l}\text { Patients who have developed structural heart disease } \\
\text { that is strongly associated with the development of } \\
\text { heart failure but who have never shown signs or } \\
\text { symptoms of heart failure }\end{array}$ & $\begin{array}{l}\text { Left ventricular hypertrophy or fibrosis; left ventricular } \\
\text { dilatation or hypocontractility; asymptomatic valvular } \\
\text { heart disease; previous MI }\end{array}$ \\
\hline C & $\begin{array}{l}\text { Patients who have current or prior symptoms of heart } \\
\text { failure associated with underlying structural heart } \\
\text { disease }\end{array}$ & $\begin{array}{l}\text { Dyspnea or fatigue due to left ventricular systolic } \\
\text { dysfunction; asymptomatic patients who are } \\
\text { undergoing treatment for prior symptoms of heart } \\
\text { failure }\end{array}$ \\
\hline D & $\begin{array}{l}\text { Patients with advanced structural heart disease and } \\
\text { marked symptoms of heart failure at rest despite } \\
\text { maximal medical therapy and who require specialised } \\
\text { interventions }\end{array}$ & $\begin{array}{l}\text { Patients who are frequently hospitalised for heart } \\
\text { failure or cannot be safely discharged from the hospital; } \\
\text { patients in the hospital awaiting heart transplantation; } \\
\text { patients at home receiving continuous intravenous } \\
\text { support for symptom relief or being supported with a } \\
\text { mechanical circulatory assist device; patients in a } \\
\text { hospice setting for the management of heart failure }\end{array}$ \\
\hline
\end{tabular}

clinical surveys of heart failure in the general population are uncommon. ${ }^{24}$ In addition to these factors, differences in sampling methods, geographical factors and case finding methods have rendered comparisons, whether regionally, nationally and internationally, difficult. ${ }^{25-27}$ Although mortality, hospital admissions and primary care data are often used to examine disease epidemiology, they are poor surrogates for assessing the incidence, prevalence and aetiology of heart failure owing to the poor validity of clinical diagnosis and the opportunity for miscoding of routine patient data and death certificates. Notwithstanding these difficulties, several studies in the USA, UK and other European countries have examined the epidemiology of heart failure. ${ }^{28-36}$

\section{Incidence}

The incidence of heart failure appears to vary, with rates differing depending on the definition of heart failure used (i.e. whether all causes of heart failure or the major causes such as congestive heart failure), the demographic composition and the socio-economic structure of the population studied. In a review of the trends in the epidemiology of congestive heart failure, Dominguez and colleagues ${ }^{35}$ found that the general incidence of congestive heart failure ranged from one to 10 cases per 1000 individuals per year. It was evident that incidence rates varied between age and sex groups (Table 5). More recent results from the Framingham Heart Study and the Rochester study have confirmed these findings. Lloyd-Jones ${ }^{34}$ reports that the age-adjusted incidence of heart failure from the Framingham Heart Study during the 1980s was estimated at 7.2 cases per 1000 in men and 4.7 cases per 1000 women aged $\geq 45$ years. Senni and colleagues ${ }^{33}$ assessed the incidence of congestive heart failure in a community in Rochester (USA) in 1981 and 1991. The age- and sex-adjusted incidence of congestive heart failure was 2.8 per 1000 personyears [standard error (SE) $0.28 ; 95 \%$ confidence interval (CI) 2.2 to 2.3; upper CI as reported in study but is incorrect] in 1981 and 2.8 per 1000 person-years (SE 0.24; 95\% CI 2.3 to 3.3) in 1991.

Studies have shown similar differences in the incidence of heart failure in the UK. Cowie and colleagues $^{24}$ examined the incidence and aetiology of heart failure in a community of 150,582 people attending 31 GP practices (82 GPs) in Hillingdon, South London (UK) in 1996. During the 20 months of the study, 220 new cases of heart failure were diagnosed among people aged 25 years and over, equating to a crude incidence of 1.3 cases per 1000 population per year. The incidence varied by age group, ranging from 0.02 cases per 1000 population per year for people 
TABLE 5 Incidence of heart failure per 1000 population

\begin{tabular}{|c|c|c|c|c|}
\hline Study & Age (years) & Men & Women & Persons \\
\hline Framingham 34 & $\begin{array}{l}45-54 \\
55-64 \\
65-74 \\
75-84\end{array}$ & $\begin{array}{l}2.0 \\
4.0 \\
8.0 \\
14\end{array}$ & $\begin{array}{l}1.0 \\
3.0 \\
5.0 \\
13\end{array}$ & \\
\hline Rochester $^{37}$ & $\begin{array}{r}45-54 \\
55-64 \\
65-74 \\
0-74\end{array}$ & $\begin{array}{r}0.8 \\
4.0 \\
13.2 \\
0.7\end{array}$ & $\begin{array}{l}1.3 \\
7.2 \\
1.6\end{array}$ & 1.1 \\
\hline Goteborg $^{31}$ & $\begin{array}{l}50-54 \\
55-64 \\
61-67\end{array}$ & $\begin{array}{r}1.5 \\
4.3 \\
10.2\end{array}$ & & \\
\hline London, UK ${ }^{24}$ & $\begin{array}{r}25-34 \\
35-44 \\
45-54 \\
55-64 \\
65-74 \\
75-84 \\
>85\end{array}$ & $\begin{array}{c}0.00 \\
0.2 \\
0.3 \\
1.7 \\
3.9 \\
9.8 \\
16.8\end{array}$ & $\begin{array}{l}0.04 \\
0.2 \\
0.1 \\
0.7 \\
2.3 \\
5.9 \\
9.6\end{array}$ & $\begin{array}{l}0.02 \\
0.2 \\
0.2 \\
1.2 \\
3.0 \\
7.4 \\
11.6\end{array}$ \\
\hline Total & & 1.4 & 1.2 & 1.3 \\
\hline
\end{tabular}

aged 25-34 years to 11.6 cases per 1000 population per year for people aged $\geq 85$ years (Table 5). The median age at presentation was 76 years (Table 5). Also, the incidence of heart failure differed significantly between males and females. Males had a higher incidence than females for those groups aged $\geq 45$ years [age-standardised incidence ratio 1.75 (95\% CI 1.34 to 2.29$), p<0.0001]$.

Johansson and colleagues ${ }^{38}$ found similar age and sex variations in incidence of heart failure in the UK, although the actual rates were higher. Using the UK General Practice Research Database, they identified patients aged $40-84$ years who were newly diagnosed with heart failure in 1996 (excluded people with previous diagnosis of heart failure or cancer and pregnant women). Of the 689,467 people included in the study, $3123 \mathrm{had}$ a new diagnosis of heart failure. Of these, 1200 were randomly sampled to validate their diagnosis, with 938 confirmed. The incidence of heart failure was 4.2 per 1000 person-years (4.4 per 1000 personyears in men and 3.9 per 1000 person-years in women). The relative risk (RR) of heart failure was 2.1 (95\% CI 1.7 to 2.6) for men compared with women $<65$ years old and 1.3 (95\% CI 1.2 to 1.4 ) aged $>65$ years. The incidence of heart failure increased with age. Lower proportions of heart and females $0.9 \%$ ) than those aged $70-79$ years (males $46.8 \%$ and females $52.1 \%$ ).

\section{Prevalence}

Dominguez and colleagues ${ }^{35}$ review of the epidemiology of congestive heart failure showed that the unadjusted prevalence rates in Europe and the USA ranged from 3 to 20 per 1000 . As with the incidence of heart failure, prevalence rates vary with age (Table 6). Prevalence rates increase from around 20 per 1000 in people aged $<45$ years to 50 per 1000 in those aged 70-79 years and over 100 per 1000 in people $>80$ years old. Recent estimates from the Framingham Heart Study in the USA show similar prevalence rates, with rates in the 1980s of 24 per 1000 men and 25 per 1000 women aged $\geq 45$ years. ${ }^{34}$ Other international studies have shown similar prevalence rates. A cross-sectional survey of 19,877 patients seeking medical care in outpatient clinics in Poland aged $>65$ years $^{39}$ identified $53 \%$ with heart failure, with higher rates among older age groups $(48 \%$ of men and $45 \%$ of women aged $65-69$ years and $69 \%$ of men and $68 \%$ of women aged $>85$ years). A study in Australia that assessed the prevalence of chronic heart failure in patients aged $\geq 60$ years attending general practitioners found that $13.2 \%$ suffered the condition. ${ }^{40}$ As previously discussed, some of the variability in these studies may result from the methods of data acquisition and diagnostic criteria. 
TABLE 6 Prevalence of heart failure (per 1000 individuals)

\begin{tabular}{|c|c|c|c|c|}
\hline Study & Age (years) & Men & Women & Total \\
\hline Framingham ${ }^{34}$ & $\begin{array}{l}50-59 \\
80-89\end{array}$ & $\begin{array}{r}8 \\
68\end{array}$ & $\begin{array}{r}8 \\
79\end{array}$ & $\begin{array}{l}7.4 \\
7.7\end{array}$ \\
\hline Tecumseh $^{44}$ & & 5.9 & 8.9 & 7.4 \\
\hline Evans County ${ }^{30}$ & & 24.8 & 17.8 & 21.2 \\
\hline NHANES-I ${ }^{28}$ & $\begin{array}{l}25-54 \\
55-64 \\
65-74\end{array}$ & $\begin{array}{r}8 \\
45 \\
49\end{array}$ & $\begin{array}{l}13 \\
30 \\
43\end{array}$ & $\begin{array}{l}11 \\
37 \\
45\end{array}$ \\
\hline Rochester $^{37}$ & $\begin{array}{r}35-54 \\
55-64 \\
65-74 \\
>75\end{array}$ & $\begin{array}{r}0 \\
5 \\
23 \\
69\end{array}$ & $\begin{array}{r}2 \\
5 \\
0 \\
80\end{array}$ & $\begin{array}{r}1 \\
5 \\
12 \\
76\end{array}$ \\
\hline Goteborg $^{31}$ & $\begin{array}{l}70 \\
75\end{array}$ & $\begin{array}{l}110 \\
170\end{array}$ & $\begin{array}{r}80 \\
110\end{array}$ & $\begin{array}{r}93 \\
136\end{array}$ \\
\hline London, UK ${ }^{29}$ & $\begin{array}{l}<65 \\
>65\end{array}$ & & & $\begin{array}{l}0.6 \\
28\end{array}$ \\
\hline Italy 32 & $\begin{array}{l}55-59 \\
60-64\end{array}$ & & & $\begin{array}{r}60 \\
114\end{array}$ \\
\hline West Midlands, UK ${ }^{36}$ & $\begin{array}{r}45-54 \\
55-64 \\
65-74 \\
75-84 \\
\geq 85\end{array}$ & $\begin{array}{r}6 \\
30 \\
48 \\
49 \\
87\end{array}$ & $\begin{array}{r}0 \\
5 \\
11 \\
26 \\
0\end{array}$ & $\begin{array}{r}3 \\
18 \\
29 \\
37 \\
30\end{array}$ \\
\hline Total & & 30 & 7 & 18 \\
\hline
\end{tabular}

Within the UK, more recent estimates of population prevalence rates for heart failure have tended to come from small sample cross-sectional surveys. Davies and colleagues ${ }^{36}$ investigated the prevalence of heart failure and left ventricular systolic dysfunction in the population served by a sample of GP practices in the West Midlands (UK) between 1995 and 1999. In a random sample of 6826 people aged $\geq 45$ years, 3960 people were screened using patient history, examination,

electrocardiography and echocardiography. Seventy-two people, $1.8 \%$ (95\% CI 1.4 to 2.3 ), had definite left-ventricular systolic dysfunction. Prevalence increased with age and was higher in males (see Table 6). Morgan and colleagues ${ }^{41}$ undertook a similar study to look at the prevalence and characteristics of heart failure (left ventricular dysfunction) within general practice in the UK, examining 817 patients between the ages of 70 and 84 years at a four-centre GP practice in Poole, Dorset (UK). Overall prevalence in this population was $7.5 \%$ (95\% CI 5.8 to 9.5). Left ventricular dysfunction was significantly $(p<0.001)$ more prevalent among men (12.8\%; $95 \%$ CI 9.6 to 16.6$)$ than women $(2.9 \%$; $95 \%$ CI 1.8 to 5.0$)$. When considering the severity of dysfunction, the prevalence of 'mild' dysfunction was $5.0 \%$, 'moderate' dysfunction $1.6 \%$ and 'severe' dysfunction $0.7 \%$. In a study of 16 general practices in England, Davis and colleagues ${ }^{42}$ studied 1062 patients with previous MI, angina, hypertension or diabetes to assess the prevalence of systolic dysfunction and heart failure. Heart failure, defined as symptoms of dyspnoea and evidence of cardiac dysfunction, was reported in $16 \%$ (95\% CI 11.6 to $21.2 \%$ ) of patients with previous MI, 8.4\% (95\% CI 5.6 to $12.0 \%)$ in those with angina and $7.7 \%(95 \%$ CI 4.5 to $12.2 \%$ ) in those with diabetes. In a survey of 10,022 registered patients in one primary care practice in Scotland, ${ }^{43} 191$ patients with heart failure were identified, relating to a prevalence rate of 19 per 1000 population. This survey was based on those with recorded symptoms and/or receiving treatment and states that the estimates will have missed symptomless patients and those with early heart failure not requiring treatment. Heart failure was most prevalent among the older age groups with a mean age of 76.9 years. In fact, the majority of cases were among those aged $\geq 65$ years for whom the prevalence was 90 per 1000 . 


\section{Heart failure: aetiology and patient characteristics}

As already indicated, people with heart failure in the UK and other developed countries have tended to suffer from coronary heart disease or hypertension, although many will have experienced several causative factors. McCallum and colleagues ${ }^{43}$ found that $58.1 \%$ of patients with heart failure registered with a general practice in Scotland were suffering from coronary heart disease, while $21.9 \%$ had hypertension. Morgan and colleagues, ${ }^{41}$ in a study of elderly heart failure patients attending a GP practice in Dorset (UK), showed through multivariate regression analysis that the strongest predictors of heart failure were a previous diagnosis of heart failure [odds ratio (OR) $5.8,95 \%$ CI 2.9 to $11.5, p<0.001$ ), being male (OR 5.1; 95\% CI 2.6 to $10.1, p<0.001$ ) and a previous diagnosis of either MI (OR 2.6; $95 \%$ CI 1.3 to $5.2, p=0.007$ ), stroke (OR 2.6; $95 \%$ CI 1.1 to $6.4, p=0.034)$ or angina (OR 2.1; 95\% CI 1.1 to $4.2, p=0.031$ ). Similar findings were described by Cowie and colleagues ${ }^{24}$ in a study of heart failure in London (UK). Coronary heart disease was thought to be the most common aetiology (36\% of patients), although half of these also suffered from hypertension (51\%). In fact, hypertension was the primary aetiology of heart failure in $31 \%$ of patients. Some $34 \%$ of patients could not have an aetiology allocated at review. The aetiology of patients did not differ significantly by sex $(p=0.11)$ or age $(p=0.65)$. Johansson and colleagues ${ }^{38}$ examined the characteristics and aetiology of 938 patients aged 40-84 years sampled from the UK General Practice Research Database who were newly diagnosed with heart failure, comparing them with a similar age and sex cohort of 5000 controls without heart failure. A history of smoking was associated with an increased risk of heart failure. Smokers (RR 2.0; 95\% CI 1.6 to 2.5) and exsmokers (RR 1.5; 95\% CI 1.2 to 2.0) were more likely to suffer from heart failure than nonsmokers. Also, heart failure patients were more likely to suffer from obesity [body mass index $(\mathrm{BMI}) \geq 30$ ] (RR 2.1; 95\% CI 1.5 to 2.8). Although there were several prior comorbities associated with heart failure, coronary heart disease had the strongest association (RR 3.2; 95\% CI 2.7 to 3.7). Davies and colleagues ${ }^{36}$ examined the epidemiology of patients aged $>45$ years with heart failure identified in a cross-sectional population survey among general practices in the West Midlands, UK. The 92 patients with definite heart failure were predominantly male $(64 \%)$ and tended to suffer from angina (41\%), hypertension $(39 \%)$, MI $(30 \%)$ or diabetes (13\%). In addition, $29 \%$ had a previous family history of MI before the age of 65 years.

\section{Prognosis of heart failure}

Heart failure often has a poor prognosis, ${ }^{45}$ with survival rates worse than for breast and prostate cancer. Despite grading heart failure by severity of symptoms, it is difficult to specify a prognosis for people with heart failure as they have a high risk of sudden death. Annual mortality for those with heart failure ranges from $10 \%$ to $>50 \%$ depending on severity. ${ }^{46,47}$ Approximately $50 \%$ of patients with a diagnosis of heart failure may die within 4 years and in patients with severe heart failure $>50 \%$ may die within 1 year. ${ }^{33,46-48}$ Cowie and colleagues stated that 5 -year mortality can range from 26 to $75 \%{ }^{48}$ There is evidence that people with heart failure have a worse QoL than people with most other common medical conditions. Psychosocial function is impaired with over onethird experiencing severe and prolonged depressive illness.

\section{Epidemiology of end-stage heart failure}

The epidemiology of ESHF has received less attention than that given to heart failure. As already discussed, the lack of information reflects the difficulties in establishing a definition, diagnostic criteria and measures of severity for heart failure. Information on incidence and prevalence of ESHF originates from studies of heart failure that have assessed the severity of disease through measures such as the NYHA functional classification. Although these studies provide some indication of the incidence and prevalence of ESHF, caution should be exercised in interpreting the information owing to differences in definitions used for heart failure, the populations included, the study designs employed and the measures of severity applied.

\section{Incidence}

Limited information was available on the incidence of ESHF (Table 7). Johansson and colleagues ${ }^{38}$ examined the severity of heart failure in 805 of 938 newly diagnosed patients aged 40-84 years sampled from the UK General Practice Research Database (for 133 patients severity was unknown). The severity of heart failure was assessed through the NYHA functional 
classification, with ESHF defined as NYHA Class IV. Some $16 \%$ of patients were identified as NYHA Class IV suffering the severe symptoms of ESHF. Of the remaining patients, $37 \%$ were in NYHA Class III suffering marked limitations on activity, $43 \%$ were in Class II with slight limitations on their activities and 5\% were in NYHA Class I suffering no limitations. A slightly higher proportion of males (17.2\%) than females (14.9\%) were classified as Class IV. In a study in the USA of the incidence of congestive heart failure, Senni and colleagues ${ }^{33}$ found that $71 \%$ of patients in 1981 and $57 \%$ in 1991 were classified as NYHA Class III or IV.

\section{Prevalence}

The studies of the prevalence of ESHF have assessed different types of heart failure and patient groups and, as a consequence, have shown variations in rates (Table 7). Two surveys of the management of heart failure in primary and secondary care within Europe provide an indication of the prevalence of different severities of heart failure. ${ }^{49,50}$ In an international survey of clinical practice in primary care (IMPROVEMENT Survey) among 15 European countries undertaken by Cleland and colleagues, ${ }^{49}$ primary care physicians kept a log of all patients they saw with heart failure or who had a MI with or without heart failure. Of the 11,062 patients included in the study, 8361 (76\%) had symptoms of heart failure and were reported to suffer breathlessness, fatigue and ankle swelling. Some $1144(10 \%)$ people were thought to have severe symptoms of heart failure. In the UK, 358 of the 559 patients surveyed $(60 \%)$ had symptoms of heart failure and were reported to have breathlessness, fatigue and ankle swelling, with 65 (11\%) having severe symptoms. Although the study provides an indication of the proportion of people with heart failure who have severe symptoms, it might provide an overestimate as these patients tend to visit primary care more frequently and practitioners may have underreported those with less severe symptoms whilst their diagnosis may have been uncertain. In a second international survey, Cleland and colleagues examined patterns of treatment and aspects of the characteristics and outcomes of patients with known or suspected heart failure admitted to hospital..$^{50}$ The survey recorded deaths and discharges from general medical, cardiology, cardiac surgery and care of the elderly wards of patients with a discharge diagnosis of acute MI or new onset atrial fibrillation or who had diabetes. Inclusion was based on patients having either a clinical diagnosis of heart failure on admission or recorded on hospital notes in the last 3 years, administration of loop diuretics for any reason other than renal failure in the 24 hours prior to discharge or administration of treatment for heart failure or major ventricular dysfunction within 24 hours of death or discharge. Some 46,788 deaths and discharges were examined, with $24 \%$ meeting the criteria for enrolment in the survey. Of these patients, 3260 who had undergone treatment and survived were asked to attend for a 12-week followup interview and physical examination. The follow-up survey found that $26 \%$ of patients were in NYHA Class III or IV, although in the UK the figure was higher with $40 \%$ in NYHA Class III or IV. The study may have overestimated the extent of heart failure owing to the broad definitions used to classify the condition and the exclusion of certain specialties.

Davies and colleagues ${ }^{36}$ examined the epidemiology of patients aged $>45$ years with heart failure identified in a cross-sectional population survey among general practices in the West Midlands, UK. Of the 3960 people surveyed, only $2 \%$ of patients were in NYHA Class IV suffering from severe symptoms of heart failure at rest. Similarly, $2 \%$ of patients experienced marked limitations on their physical activity and were classified as being in NYHA Class III. The majority of patients were in NYHA Class I (82\%) or Class II (14\%), incurring no or slight limitations on their activities. Of the 92 patients with definite heart failure, $16 \%$ were in Class IV and $15 \%$ in Class III. In another study of patients with previous MI, angina, hypertension or diabetes in 16 general practices in England, Davis and colleagues ${ }^{42}$ found similar prevalence rates for ESHF, with 3\% of patients in Class IV and 6\% in Class III. Morgan and colleagues ${ }^{41}$ assessed the severity of heart failure among patients aged 70-84 years attending GP practices in Poole, Dorset (UK), undertaking a qualitative assessment of left ventricular dysfunction. Although the majority of patients were thought to have normal left ventricular function $(92.7 \%)$ or mild dysfunction (5\%), 1.6\% had 'moderate' dysfunction and $0.7 \%$ had 'severe' dysfunction. Similar rates of ESHF were found in a cross-sectional survey of patients aged $\geq 65$ years in Poland, with $6 \%$ of men and $4 \%$ of women in NYHA Class IV and $32 \%$ of men and $30 \%$ of women in Class III. ${ }^{39}$ Studies in Italy of elderly hospitalised patients or patients attending hospital due to heart failure showed a higher prevalence of ESHF. Rengo and colleagues $^{51}$ found that $9.6 \%$ of patients in a cohort of elderly hospitalised patients (aged $\geq 65$ years) were in NYHA Class IV and $24.4 \%$ in 
TABLE 7 Percentage of patients with heart failure by severity classifications (NYHA functional classification unless stated otherwise)

\begin{tabular}{|c|c|c|c|c|c|}
\hline \multirow[t]{2}{*}{ Study } & & \multicolumn{4}{|c|}{ NYHA classification } \\
\hline & & $\mathbf{I}$ & II & III & IV \\
\hline \multicolumn{6}{|l|}{ Incidence } \\
\hline Johansson et al., $2001^{38}$ & Persons & 4.6 & 42.7 & 36.6 & 16.0 \\
\hline Senni et al., $1999^{33}$ & $\begin{array}{l}1981 \\
1991\end{array}$ & \multicolumn{2}{|c|}{29} & \multicolumn{2}{|c|}{71} \\
\hline \multicolumn{6}{|l|}{ Prevalence } \\
\hline Davies et al., 200I ${ }^{36}$ & Persons $\geq 45$ years & 82 & 14 & 2 & 2 \\
\hline Davis et al., $2002^{42}$ & Persons & 59 & 32 & 6 & 3 \\
\hline Rengo et al., $1996^{51}$ & Persons $\geq 65$ years & 25.6 & 40.5 & 24.4 & 9.6 \\
\hline Tavazzi et al., $1997^{52}$ & Persons & 6.9 & 43.8 & 36.0 & 12.6 \\
\hline Albanese et al., $1999^{53}$ & Persons & 3 & 19 & 28 & 50 \\
\hline \multirow[t]{2}{*}{ Rywik et al., $2000^{39}$} & Men & 14.8 & 47.8 & 31.7 & 5.7 \\
\hline & Women & 12.4 & 53.4 & 30.0 & 4.2 \\
\hline Morgan et al., I9994Ia & Persons $70-84$ years & 92.7 & 5.0 & 1.6 & 0.7 \\
\hline Cleland et al., 2002 $49 b$ & Persons & 12 & 34 & 31 & 10 \\
\hline Cleland et al., $2003^{50}$ & Persons & 36 & 37 & \multicolumn{2}{|c|}{26} \\
\hline \multicolumn{6}{|c|}{$\begin{array}{l}{ }^{a} \text { Severity was assessed as either no, mild, moderate or severe dysfunction rather than NYHA functional classification I, II, III } \\
\text { and IV. } \\
\text { b Severity was assessed as either asymptomatic, mild, moderate or severe rather than NYHA functional classification I, II, III } \\
\text { and IV, with I } 2 \% \text { having no severity recorded and } 3 \% \text { dead. }\end{array}$} \\
\hline
\end{tabular}

Class III. The SEOSI study in Italy, ${ }^{52}$ which examined the characteristics of patients with suspected or known heart failure attending 395 cardiology units, found that $12.6 \%$ of patients were in NYHA Class IV and 36\% in Class III. Albanese and colleagues ${ }^{53}$ study of CHF patients admitted to a hospital in Italy found that $50 \%$ were in NYHA Class IV, 28\% in Class III and 22\% in Class I or II.

\section{End-stage heart failure and the needs and demands for healthcare}

Despite the lack of evidence on the epidemiology of heart failure, the information on the incidence and prevalence of ESHF found provides some indication of the likely need and demand for treatment with either heart transplantation or mechanical support. The review of the epidemiology of heart failure showed widely differing incidence and prevalence rates depending on the nature of the study and the structure of the populations examined. Using studies of the incidence and prevalence of heart failure and population estimates relevant to England and Wales, it was possible to estimate the people who might suffer from ESHF (Table 8). Estimates of prevalence suggest that there would probably be between 250,000 and 400,000 people with heart failure in England and Wales, with approximately $7000-8000$ people with ESHF. Incidence rates suggest that there would be between 50,000 and 100,000 new cases of heart failure annually, with approximately 10,000-15,000 new cases of ESHF. The disparity in the prevalence and the incidence of ESHF reflects the poor prognosis for sufferers. The majority of people with ESHF have the condition for a short period and die, with only a small number receiving treatment and surviving.

Estimates from the incidence and prevalence of ESHF provide a crude estimate of the population that may benefit from the different treatment options available, specifically heart transplantation or support with a mechanical device. Identifying those who would benefit from an LVAD as a BTT, BTR or LTCS is more difficult. UK transplant and waiting list figures give some indication of the potential numbers of LVAD recipients, but these do not represent the actual need. With limited and decreasing numbers of organ donors and, as a consequence, the managed and selective nature of transplant waiting lists, these data provide a significant underestimate of actual demand, let alone the actual need. However, attempts have 
TABLE 8 Estimate of the number of patients with ESHF in England and Wales (NYHA functional classification unless stated otherwise)

\begin{tabular}{|c|c|c|c|c|c|}
\hline & $\begin{array}{l}\text { Incidence/ } \\
\text { prevalence of } \\
\text { heart failure } \\
\text { (per } 1000 \text { people) }\end{array}$ & $\begin{array}{l}\text { Population in } \\
\text { England and } \\
\text { Wales } \\
\text { (mid-2002) }^{54}\end{array}$ & $\begin{array}{l}\text { No. of heart } \\
\text { failure patients }\end{array}$ & $\begin{array}{l}\text { Percentage of } \\
\text { heart failure } \\
\text { patients in } \\
\text { NYHA Class IV }\end{array}$ & $\begin{array}{l}\text { No. of ESHF } \\
\text { patients }\end{array}$ \\
\hline \multicolumn{6}{|l|}{ Incidence } \\
\hline $\begin{array}{l}\text { UK - persons } \\
(40-84 \text { years })^{38}\end{array}$ & 4.2 & $23,710,000$ & 99,582 & 16.0 & 15,933 \\
\hline $\begin{array}{l}\text { UK - persons } \\
\text { (all ages) }^{24}\end{array}$ & 1.3 & $52,455,300$ & 68,192 & $16.0^{55}$ & 10,911 \\
\hline \multicolumn{6}{|l|}{ Prevalence } \\
\hline $\begin{array}{l}\text { UK - persons } \\
(>45 \text { years })^{36}\end{array}$ & 18 & $20,944,000$ & 376,992 & 2 & 7,540 \\
\hline $\begin{array}{l}\text { UK - persons } \\
(70-84 \text { years })^{4 l a}\end{array}$ & 75 & $5,059,000$ & 379,425 & 0.7 & 2,656 \\
\hline $\begin{array}{l}\text { UK - persons } \\
\text { (<65 years) }\end{array}$ & 0.6 & $44,069,000$ & $26,44 I$ & $3^{42}$ & 7,837 \\
\hline $\begin{array}{l}\text { Persons } \\
(\geq 65 \text { years })^{35}\end{array}$ & 28 & $8,386,000$ & 234,808 & & \\
\hline
\end{tabular}

TABLE 9 Definitions of heart transplant concepts ${ }^{56}$

Unrecognised need - the number of suitable people never placed on the transplant waiting list

Unmet demand - the number of people awaiting transplantation plus the number of people who die while on the waiting list

Met demand - the number of transplantations performed

Unmet need - the number of suitable people never placed on the transplant waiting list plus the number of people awaiting transplantation plus the number of people who die while on the waiting list

Total demand - the number of people awaiting transplantation plus the number of people who die while on the waiting list plus the number of transplantations performed

Total need - the number of people never listed for transplantation plus the number of people awaiting transplantation plus the number of people who die on the waiting list plus the number of transplantations performed

been made to try to identify the extent of the problem presented by ESHF.

In a study of cardiac replacement, Evans ${ }^{56}$ examined the need and demand for and supply of heart transplants within the USA. The study identified six key concepts in defining the need and demand for heart transplantation and the relationships that exist between need, demand and supply (Table 9). Need for heart transplantation was identified as those people with diagnoses of specific cardiac conditions as the primary cause of death that were considered suitable for heart transplantation (Table 10). It is likely that this may represent an overestimate as people may have had other conditions that would have rendered them unsuitable candidates for transplantation or they may have died suddenly without opportunity for transplant. The actual supply of donor hearts was recognised by Evans ${ }^{56}$ to represent only a fraction of the potential supply, up to $50 \%$, owing to the failure to obtain consent from relatives. Based on these relationships, Evans ${ }^{56}$ was able to estimate the need, demand and supply within the USA (Table 11).

Applying the general principles established by Evans, ${ }^{56}$ estimates were made for the UK of the need and demand for heart transplantations using two different approaches. The first approach employed the definitions of need, demand and supply (Table 9) and the mathematical relationships 
TABLE 10 Diagnoses at death for which cardiac transplantation could have been indicated ${ }^{57}$

\begin{tabular}{|ll|}
\hline ICD-9 code & Description \\
\hline 164.1 & Malignant neoplasm of the heart \\
394.0 & Mitral valve stenosis \\
394.1 & Rheumatic mitral insufficiency \\
394.2 & Mitral stenosis with insufficiency \\
394.9 & Other and unspecified mitral valve disease \\
395.0 & Rheumatic aortic stenosis \\
395.1 & Rheumatic aortic insufficiency \\
395.2 & Rheumatic aortic stenosis with insufficiency \\
395.9 & Other and unspecified rheumatic aortic diseases \\
396 & Diseases of mitral and aortic valves \\
398.0 & Rheumatic myocarditis \\
398.9 & Other and unspecified rheumatic heart diseases \\
414.0 & Coronary atherosclerosis \\
414.1 & Aneurysm of the heart \\
414.8 & Other specified forms of chronic ischaemic heart disease \\
424.1 & Aortic valve disorders \\
425.0 & Endomyocardial fibrosis \\
425.4 & Other primary cardiomyopathies \\
428.0 & Congestive heart failure \\
428.1 & Left heart failure \\
428.9 & Heart failure, unspecified \\
429.0 & Myocarditis, unspecified \\
429.1 & Myocardial degeneration \\
429.3 & Cardiomegaly \\
\hline$a$ Each condition must be listed as the primary cause of death. Excludes indications for paediatric heart transplantation. \\
\hline
\end{tabular}

TABLE II UK projections of Evans' need, demand and supply

\begin{tabular}{|c|c|c|c|c|c|}
\hline & \multirow{2}{*}{$\begin{array}{l}\text { USA }^{56} \\
1995\end{array}$} & \multirow[t]{2}{*}{ Relative weight } & \multicolumn{3}{|c|}{$\mathbf{U K}^{a}$} \\
\hline & & & 1995 & 2003 & 2000 \\
\hline Total need & 50,204 & 21.26 & 6,782 & 3,146 & $34,329^{b}$ \\
\hline Total demand & 6,599 & 2.80 & 893 & $4 / 4$ & $402^{c}$ \\
\hline Unmet demand & 4,238 & 1.80 & 574 & 266 & $198^{c}$ \\
\hline Transplants (met demand) & 2,361 & 1.00 & $319^{c}$ & $148^{c}$ & $204^{c}$ \\
\hline Actual donor supply & 2,506 & 1.06 & 338 & 157 & $224^{c}$ \\
\hline Potential donor supply & 8,749 & 3.71 & 1,183 & 549 & 756 \\
\hline Unmet need & 47,843 & 20.26 & 6,463 & 2,998 & 34,125 \\
\hline Waiting list & 3,468 & $\mathrm{I} .47$ & 469 & 218 & $156^{c}$ \\
\hline Unrecognised need & 43,605 & 18.47 & 5,892 & 2,734 & 33,927 \\
\hline
\end{tabular}

between the different categories in the USA to produce relative weights (Table 11) that could be applied to known variables, such as the number of heart transplants, to estimate the need, supply and demand in the UK. Heart transplant data for the UK in 1995 (Table 11) were used so as to be consistent with the base year used by Evans. With 319 people receiving a heart transplant, it was estimated that there could be some 6782 people who could need a heart transplant, although only 893 people would demand treatment. This suggests an unmet demand of 574 people and unmet need of 6463 people. When compared with the estimates of the prevalence of ESHF (Table 8), it is evident that the estimates for the UK from populating the model developed by Evans are relatively similar at around 7000 patients. A second set of estimates were calculated using heart 
TABLE 12 Number of deaths in England and Wales in 2000 from conditions identified by Evans ${ }^{56}$ as the main indications for adult heart transplantation ${ }^{58}$

\begin{tabular}{|c|c|c|c|c|}
\hline ICD-9 & Description & Male & Female & Total \\
\hline 164.1 & Malignant neoplasm of the heart & 6 & 4 & 10 \\
\hline 394.0 & Mitral valve stenosis & 24 & 155 & 179 \\
\hline 394.1 & Rheumatic mitral insufficiency & 4 & 8 & 12 \\
\hline 394.2 & Mitral stenosis with insufficiency & I & 5 & 6 \\
\hline 394.9 & Other and unspecified mitral valve disease & 213 & 552 & 765 \\
\hline 395.0 & Rheumatic aortic stenosis & 7 & 6 & 13 \\
\hline 395.1 & Rheumatic aortic insufficiency & I & 0 & I \\
\hline 395.2 & Rheumatic aortic stenosis with insufficiency & 0 & 0 & 0 \\
\hline 395.9 & Other and unspecified rheumatic aortic diseases & 5 & 5 & 10 \\
\hline 396 & Diseases of mitral and aortic valves & 79 & 259 & 338 \\
\hline 398.0 & Rheumatic myocarditis & 0 & 0 & 0 \\
\hline 398.9 & Other and unspecified rheumatic heart diseases & 30 & 102 & 132 \\
\hline $4 \mid 4.0$ & Coronary atherosclerosis & I I,049 & 6,733 & 17,782 \\
\hline $4 \mid 4.1$ & Aneurysm of the heart & 41 & 15 & 56 \\
\hline 414.8 & Other specified forms of chronic ischaemic heart disease & 564 & 423 & 987 \\
\hline 424.1 & Aortic valve disorders & 1,103 & $\mathrm{I}, 146$ & 2,249 \\
\hline 425.0 & Endomyocardial fibrosis & I & 0 & I \\
\hline 425.4 & Other primary cardiomyopathies & 834 & 427 & 1,261 \\
\hline 428.0 & Congestive heart failure & 2,264 & 4,011 & 6,275 \\
\hline 428.1 & Left heart failure & 553 & 878 & $|, 43|$ \\
\hline 428.9 & Heart failure, unspecified & 335 & 567 & 902 \\
\hline 429.0 & Myocarditis, unspecified & 112 & 92 & 204 \\
\hline 429.1 & Myocardial degeneration & 309 & 1,026 & 1,335 \\
\hline \multirow[t]{2}{*}{429.3} & Cardiomegaly & 211 & 169 & 380 \\
\hline & Total & 17,746 & 16,583 & 34,329 \\
\hline
\end{tabular}

transplant data for the UK for 2003. As the number of heart transplants in the UK had decreased in 2003 to $<50 \%$ of the number undertaken in 1995, estimates of need and demand have consequently fallen to unrealistic levels.

A second approach was taken to estimate need, demand and supply for heart transplants. Using the definitions identified by Evans, data were collected to populate the different components of the model. Using diagnoses at death identified by Evans as the main indications for adult heart transplantation, it was estimated that 34,329 people in England and Wales in 2000 would need a heart transplantation (Table 12). In the UK in 2000, 402 demanded a heart transplant with only 204 undergoing a transplant, meaning an unmet demand of 198 people and an unmet need of over 34,000 people (Table 11).

Some caution should be exercised when interpreting these estimates. First, opinions may differ as to the underlying conditions that might identify people as potential candidates for heart transplantation and the proportions of these people who may actually meet the criteria for heart transplantation or mechanical support.
Second, Evans used mortality data based on the primary cause of death to estimate the total need for heart transplantations. In England and Wales, mortality data are based on the underlying cause of death rather than the primary cause and, as a consequence, it is likely that the estimates for these conditions in England and Wales, will far exceed those expected given the definition used in the USA. Third, the estimates of need and demand are based on experiences within the USA, which operates a different health economy to that within the UK; as a consequence, it is likely that the relationship between the need, demand and supply of heart transplantation will differ. Fourth, the estimates are of the need and demand for heart transplantation. Although these patients may also benefit from the use of mechanical devices, other people who do not meet the criteria for transplantation may benefit from mechanical devices. It is difficult to estimate the number of patients who would fall into this category, although they are likely to be a relatively small group of people with ESHF who are considered too old for transplantation or whose condition has worsened to the extent that they would not survive heart transplantation. Fifth, the UK government is endeavouring to increase organ donations, but although this may increase supply, current practice 
suggests that any increase is unlikely to be substantial. Patterns of heart donation have been in decline over the last 20 years in England and Wales (see Table 1). Sixth, the intention was to assess the likely need, demand and supply for heart transplantation in England and Wales. The UK Transplant Authority publishes heart transplantation data for the UK, whereas the mortality and population data are for England and Wales. As a consequence, the heart transplant data and any calculated data based on such data may be an overestimate if applied to England and Wales.

Irrespective of these possible limitations, it is evident from the incidence and prevalence of ESHF, the patterns of heart transplantation in the UK and the estimates of need and demand for treatment of ESHF in England and Wales that there is a large potential need for treatment that could be addressed through the use of other forms of treatment, such as mechanical assist devices. It is likely that there are some $6000-8000$ people who could benefit from treatment in England and Wales currently, with as many as 10,000-15,000 new cases of ESHF annually. Given the age distribution of cases of heart failure and ESHF, most of these patients will be elderly (aged $>65$ years). Current guidelines for heart transplantation recommend that candidates should be limited to those in the younger age groups and/or who do not suffer from serious co-morbidities that may limit the success of the heart transplantation. As a consequence, it is this group of younger patients who will be considered for implantation of an LVAD as a BTT. In contrast, the elderly patients with ESHF who are not candidates for heart transplantation and for implantation of an LVAD as a BTT provide a relatively large group for whom consideration would need to be given as to whether they should receive an LVAD as an LTCS or the alternative of best supportive or palliative care. With limited guidance concerning the use of LVADs as an LTCS, it will be important to consider the benefits and costs of their use for the different patient groups whether defined by age, co-morbidity or indications for treatment and the alternatives available.

\section{Current service provision for heart failure}

Recent years have seen considerable progress in the effectiveness of treatments available for heart failure that control symptoms, reduce treatmentrelated adverse events, improve QoL and slow the progression of the syndrome, so extending patients' length of survival. ${ }^{2}$ The changing epidemiology of heart failure, a partial consequence of an ageing population and the effectiveness of the different treatments, has led to an increased need and demand for health services for heart failure. Unfortunately, differences remain as to the availability of these services and the quality of care provided in terms of geography, age, gender and ethnic group. ${ }^{2}$ Increased efforts to tackle coronary heart disease have resulted in the development of a National Service Framework to address these issues as part of the 10-year modernisation of the NHS in England and Wales. ${ }^{2}$ This section briefly outlines the treatment options used to manage people suffering from heart failure; it does not discuss their effectiveness.

Clinical management of heart failure focuses on the diagnosis of the condition suffered and establishing appropriate strategies either to treat the underlying cause (e.g. ischaemic heart disease) or to relieve symptoms of the condition (e.g. breathlessness) and/or any other precipitating factors (e.g. infections, arrhythmias). Treatment strategies will reflect the nature and the severity of the condition suffered. Usually management of the condition will intend to relieve symptoms, improve QoL and functional capacity and improve prognosis. However, palliative care may be the primary focus for some patients nearing the end of their lives and in such instances symptom relief should be accompanied by psychological and other forms of support. For other patients, the clinical management of their heart failure will involve drug therapy and, in some instances, heart transplantation or mechanical support.

As previously discussed, the reduced pump function in heart failure activates adaptive responses in the body which initially help to maintain cardiac output. However, these mechanisms often only maintain adequate cardiac output at rest, and eventually can drive the progression of cardiac deterioration. Therapy for heart failure aims to inhibit these responses. The main symptoms of CHF are breathlessness (dyspnoea) and fluid retention in tissues (oedema). Clinical manifestations are an enlarged heart and liver and a high venous pressure. The ejection fraction is reduced. Treatments for CHF also aim to improve these signs and symptoms.

First-line treatment for mild/asymptomatic CHF is with ACE inhibitors. ${ }^{2}$ These inhibit the conversion of angiotensin I to angiotensin II. Angiotensin II causes vasoconstriction, and prevention of its 
production thereby promotes vasodilation. This vasodilation reduces pulmonary congestion, oedema and cardiac workload and slows CHF progression, lengthens survival time and improves haemodynamic parameters. ${ }^{59}$ The restriction of activity and reduction of dietary sodium can also help to lessen cardiac workload and fluid retention. In moderate/symptomatic CHF, additional treatments include diuretics, cardiac glycosides and beta-blockers. Diuretics reduce fluid retention by increasing the excretion of renal salt and water and can provide rapid symptomatic relief. $^{2}$ Cardiac glycosides (most often digoxin) act on the sodium pump in cardiac muscle and enhance contractility, and slow the heart. These do not reduce mortality, but can reduce symptoms and hospital admission for worsening heart failure. ${ }^{2}$ Beta-blockers block the action of adrenaline and can relieve stress to the heart muscle and in people with controlled heart failure can reduce mortality. ${ }^{60}$

When existing therapy fails to control symptoms adequately and/or heart failure is severe, then other categories of vasodilators can also be given, for example isosorbide dinitrate and hydralazine. These have been shown to prolong survival, ${ }^{61}$ although not as effectively as ACE inhibitors. ${ }^{62}$ The addition of the diuretic spironolactone can also reduce mortality and morbidity. ${ }^{2}$ Additionally inotropic sympathomimetics and positive inotropes may also be used, although their longterm use is not beneficial. ${ }^{60}$ The cardiac stimulants dobutamine and dopamine act on $\beta_{1}$-receptors in cardiac muscle and increase contractility with little effect on rate. Positive inotropic drugs such as milrinone increase the force of contraction of the myocardium. In patients whose heart failure is refractory to treatments, LVADs and/or cardiac transplantation are also options. Palliative care and home oxygen are also treatments that are offered to severe heart failure and ESHF patients.

\section{Description of LVADs}

\section{Brief history of the development of LVADs $^{8}$}

Mechanical systems to support circulation evolved soon after the first use of coronary pulmonary bypass (CPB) in the 1950s. It followed the growth of cardiac surgical procedures which established a need for developing a means of extending circulatory support for patients who could not be weaned from CPB following cardiac surgery. The intra-aortic balloon pump (IABP) was developed in the late 1960 s as a form of partial cardiac assist and used in the early 1970s to support patients who could not be weaned from CPB. The early 1960s also saw the introduction of the first ventricular assist devices (VADs), evolving from a simple pneumatically actuated silicone-rubber tube pump to a pneumatically driven diaphragm pump. During the same period the potential benefits of non-pulsatile blood pumps (e.g. reduced complexity, smaller size and lower power needs), first recognised in the 1930s, were being developed into blood pumps.

The importance of mechanical support was recognised in the USA with the National Institute of Health setting up in 1964, following recommendations of the National Heart Advisory Group, the Artificial Heart Program. Initial focus was on the development of a total artificial heart (TAH). By 1970, the program was renamed the Medical Devices Applications Branch within the National Heart and Lung Institute and its efforts were refocused towards developing and evaluating LVADs. A programme of sponsored research was developed over the following 20 years, investigating different types of early device (e.g. pneumatically driven sac pumps, axiosymmetric pumps and tether-free pumps), the components of pumps (i.e. pumps, energy converters, controllers and power transmission) and implantable integrated electrically powered LVADs. The programme led in 1989 to the development of plans to evaluate clinically the Novacor system, the first of the LVADs to achieve the preclinical criteria. Although this study was not undertaken, further initiatives were funded in 1996 to develop long-term 'improved' VADs including firstgeneration rotary devices, two axial flow (Nimbus/University of Pittsburgh, Jarvik Heart/Texas Heart Institute), one centrifugal device (CCF/Ohio state University) and a nonblood-contacting mechanical 'muscle' wrap pump (Abiomed/Columbia Presbyterian Hospital), a conditioned skeletal muscle-powered pump (Whalen Biomedical/University of Utah) and an electromechanical left ventricular assist system (LVAS) (PSU/Arrow).

Although a VAD was used in 1978 in an attempt to bridge a patient to transplantation, it was in 1984 that two patients were bridged to transplantation with a Novacor implantable electric LVAD and a paracorporeal pneumatic Thoratec VAD and in 1988 that the HeartMate implanted pneumatic (IP) VAD was first used successfully as a BTT. These early devices were limited to console-based power and control, limiting patient care to within hospitals despite their clinical rehabilitation. The 
TABLE I3 Blood pumps

\begin{tabular}{|c|c|c|}
\hline \multirow[t]{2}{*}{$\begin{array}{c}\text { Displacement blood pumps } \\
\text { Pulsatile LVADs } \\
\text { First-generation devices }\end{array}$} & \multicolumn{2}{|c|}{$\begin{array}{l}\text { Rotary blood pumps } \\
\text { Continuous flow devices } \\
\text { Second-generation devices }\end{array}$} \\
\hline & Axial flow pumps & Non-axial flow pumps \\
\hline $\begin{array}{l}\text { HeartMate, Novacor, LionHeart, } \\
\text { Thoratec, Medos, Berlin Heart, } \\
\text { Abiomed, Nippon Zeon, Toyobo }\end{array}$ & $\begin{array}{l}\text { Jarvik } 2000 \text { Heart, } \\
\text { MicroMed DeBakey }\end{array}$ & $\begin{array}{l}\text { HeartQuest } \\
\text { Terumo }\end{array}$ \\
\hline
\end{tabular}

early 1990s saw attempts to move patients to outpatient care, improving QoL and reducing costs of care. The HeartMate vented electric (VE) LVAD in 1991 and the Novacor LVAS in 1993 using battery power provided support for patients to transplantation, allowing them greatly improved mobility. Second-generation untethered rotary pump LVADs were first introduced in 1998 with the axial flow DeBakey VAD. Subsequently, the Jarvik 2000 in 1999 and HeartMate II LVADs in 2000 have been used to support patients to heart transplantation. The developments in LVADs as a BTT has led to patients returning to more normal activities of life and being supported for longer periods on the transplant waiting list. With the shortage in donor hearts for transplantation and the improved survival and QoL of patients on mechanical support, the use of LVADs as a means of permanent support has become a possibility. Several studies of LVADs as a permanent support have been initiated. A randomised controlled trial (RCT) of the Novacor VAS was started in 1994 but failed to progress past the pilot phase. The Randomized Evaluation of Mechanical Assistance in Treatment of Chronic Heart Failure (REMATCH) trial assessing the HeartMate VE LVAS began in 1996 and has reported outcomes at 2 years' follow-up. Other studies of the HeartMate IP LVAD (PHADE, 1999), the Novacor wearable LVAD (INTrEPID, 2000), the Arrow LionHeart LVAS and the Jarvik 2000 for permanent support are under way. Several devices have received US Food and Drug Adminstration (FDA) approval and/or CE marking for BTT and/or permanent support. For BTT the HeartMate IP (1994) and VE (1998) LVADs, Thoratec VAD (1996) and Novacor wearable LVAS (1998) received FDA marketing approval in the USA. European CE marking was usually received prior to FDA approval. The HeartMate VE and the Novacor wearable LVADs are commercially approved and currently available for permanent support. The LionHeart LVAS, DeBakey, Jarvik 2000, HeartMate II, Thoratec, Berlin Heart and Toyobo VADs are undergoing clinical trials.

\section{Description of LVADs included in the systematic review and economic evaluation}

Although some 20 or more devices have been developed and used as LVADs, it is evident that some are either targeted at specific patient groups not included in the study (e.g. paediatric devices) and others have not progressed past their early development stages or are at too early a stage of development to have undergone assessment of efficacy. To ensure that the systematic review focused on those devices that are relevant to the NHS in England and Wales, the expert advisory group was asked to identify and justify those devices that should be included. The list of devices included in the review is shown in Tables 13 and 14 and the full list of devices is given in Appendix 1 . The remainder of this section discusses the devices by category/type of device, identifying their characteristics, the method of placement and licensing or marketing approval. It does not provide a detailed description of the mechanical components of the devices or of the differing implantation and explantation techniques used. Details of some devices were fairly limited, whether through publications, company websites or contact with the particular manufacturers. As a consequence, the information presented on the different devices may vary in completeness, and for some devices no information was available. It was evident throughout the assessment of LVADs for ESHF that the categorisation and terminology used to identify and describe the various devices differ. LVADs may be displacement pulsatile blood pumps or rotary continuous flow devices, whether axial or non-axial flow pumps. Some have classified the devices as either extracorporeal, paracorporeal or implantable, and others have differentiated devices as being first- or secondgeneration devices. Within this study, devices will be referred to as either first- or second-generation devices based on the specifications outlined in Tables 13 and 14. The use of the terminology is not intended to convey any predetermined assessment of clinical and cost-effectiveness, but 
rather to differentiate between devices on several characteristics. Any assessment of the clinical and cost-effectiveness of the different devices will be based on evidence for those devices for the different indications.

\section{Displacement pulsatile LVADs}

The most widely used displacement, pulsatile devices have been the Abiomed, HeartMate, Novacor and Thoratec LVADs. Such devices are connected to the patient's left ventricle and provide circulatory support by taking over the workload of the failing heart. An inflow conduit directs blood from the left ventricle into the blood pump. The external control system triggers pumping by a pusher plate mechanism, which is either electrically or pneumatically driven. The pump ejects blood through an outflow conduit to the body's arterial system via the aorta or arterial system. Bioprosthetic heart valves dictate the direction of flow. This mechanism mimics the native left ventricle by providing a pulsatile stroke volume while the patient's own left ventricle is completely off-loaded. The system is operated and monitored by a controller and powered by an external source. Implanted pumps are connected by percutaneous leads through the patient's skin. The system can be self-regulating in response to a patient's needs or use a fixed pump rate. There are differences in the configuration of the displacement pulsatile devices in terms of the position of the pump (i.e. extracorporeal, paracorporeal or implantable), implantation position (e.g. intraperitoneally or preperitoneal pocket), method of driving the mechanism (i.e. IP or VE), type of power source (i.e. wall-mounted, console-based or battery packs), positioning of cannulae and leads delivering the power and valve structure and the nature of the internal surfaces of the devices. Although the original large external pneumatic consoles have been replaced by implantable electric systems with a portable controller and power source, the serious problems of LVAD size, noise, driveline infection and thromboembolism persist. As a consequence, the next generation of displacement devices are likely to be totally implantable systems without the need for percutaneous power or communication in an attempt to solve some of these issues (e.g. Arrow LionHeart).

\section{HeartMate LVADs (Thoratec Corporation, USA) ${ }^{63}$}

Development of the HeartMate LVAD began during the late 1960s, with the first implantable LVAD with pneumatic driver and the first integrated implantable electric LVAD emerging in the early to mid $1970 \mathrm{~s}$. Initial clinical trials led to the FDA awarding an investigational device exemption for the pneumatic pusher plate HeartMate device. The first clinical implant of the device was in 1986 at the Texas Heart Institute. In 1990 the FDA awarded an investigational device exemption for the electric HeartMate device, with its first clinical implant taking place shortly afterwards. A portable, wearable driver was developed in 1995 to allow patients to be supported as outpatients and to enhance their QoL. In 1997, the first patient was discharged home with an implantable LVAD.

The HeartMate LVAD is an implantable pulsatile LVAD available as either a pneumatically driven (HeartMate IP) or an electrically powered (HeartMate VE) device. Although sharing some components and similar flow characteristics, the HeartMate IP and VE are different in aspects of their operation. The HeartMate IP has a maximum stroke volume of 83 litres, a maximum pump rate of 140 beats per minute (bpm) and flow rates of up to 12 litres/minute. It is an implantable VAD placed in the intracorporeal position to support the left ventricle and all bloodtouching surfaces are HeartMate textured surfaces designed to reduce the risk of clot formation. Percutaneous cannulae connect to an air-driven pump which is powered by an external drive console. It can be transported on a wheeled cart, but is for in-hospital use only. It has a regulator that is designed to respond to changing flow demands of the body; if activity increases, a change in flow is triggered. There is also a fixed mode with a preset beat rate. The HeartMate VE is an implantable VAD for the left ventricle with percutaneous cannulae, suitable for home use. It has a capacity of 10 litres/minute, a maximum stroke volume of $83 \mathrm{ml}$, and a pump rate of up to $120 \mathrm{bpm}$. It is designed to respond to changing flow demands of the body. A preset beat is also available. It is powered by a power base unit and wearable battery, although a belt-worn system controller and batteries can provide approximately 6 hours of mobility. HeartMate textured surfaces reduce the risk of clot formation. The HeartMate devices have external back-up mechanisms so that reoperation may not be required in the event of device failure. With the control unit externally sited, it is possible to rectify problems outside the body. In addition, a hand-held portable pump provides a means of operating the pusher plate mechanism if the motor device should fail. The HeartMate IP and VE LVADs are licensed for BTT in Europe and the USA, and the HeartMate VE LVAD is also licensed in Europe for permanent support in patients with CHF. 


\section{Novacor (World Heart Inc., USA) ${ }^{64}$}

The Novacor LVAS was the first electrically powered heart assist device designed as an integrated, implantable system for definitive treatment of ESHF. The device consists of a pump/drive unit, an electronic controller with rechargeable battery, a subcutaneous belt skin transformer secondary for transcutaneous power transfer across the intact skin and variable-volume compensator. The Novacor LVAS is placed in the intracorporeal position and used to support the left ventricle with percutaneuos cannulae. It is selfregulating, responding and adjusting instantaneously to the patients' heartbeat and circulatory demand. Stroke volume is $70 \mathrm{ml}$ and capacity is 8.5 litres/minute. The system has continued to evolve from the first systems developed in the early 1970s. In 1984, a partially implantable version of the Novacor system with a bedside console system was used as a BTT for a male patient. By 1993 a wearable controller and battery pack was developed to improve mobility and patient QoL. The current system, the Novacor N100PC, received regulatory approval as a BTT in Europe in 1993 and was first implanted in that year as a BTT. Regulatory approval in the USA for BTT came in 1998 following a multi-centre study and was also approved in Canada and Japan in 1999. In addition, it has received regulatory approval for long-term therapeutic use in Europe and the USA.

\section{Abiomed BVS 5000 (ABIOMED Cardiovascular Inc., USA) ${ }^{65}$}

The Abiomed BVS 5000 was the first cardiac assist device approved by the FDA in 1992. Currently the device is approved by the FDA for all forms of recoverable heart failure, including as a BTR or BTT in failed transplant. Its most common indication for use is short-term (10 days) postcardiotomy support (recovery after heart surgery). It is a dual-chamber VAD placed outside the body (extracorporeal) and is suitable for shortterm left, right, or biventricular support. The Abiomed BVS 5000 consists of three components: a transthoracic cannula, disposable external (bedside) pumps and an automated self-regulating pulsatile microprocessor-controlled pneumatic drive console. The device has a constant stroke volume of $\sim 80 \mathrm{ml}$ and a maximum output of 6 litres/minute if patient hydration is adequate. It is driven by a pneumatic drive console which is large in size and suitable for in-hospital use only. Some limited mobility is available with the battery providing 1 hour of support, which also acts as a safety backup system. In addition, it has a foot pump for manual operation. The VAD is fully automatic and compensates for changes in preload and afterload and is asynchronous to the native cardiac rhythm. In biventricle use the left and right ventricles are triggered independently of each other. The Abiomed BVS 5000 was developed with the aim of being a safe, simple and effective device. The safety of the device comes from its design, incorporating a dual-chamber pump and built-in reservoir with blood draining through gravity, avoiding the need for a vacuum. The simplicity is based on its automated control systems, requiring minimal operator input. The device has certain limitations, including restricted device mobility, limited flow capability and the need for full anticoagulation due to thrombus formation.

\section{Thoratec VAD and IVAD (Thoratec Corporation, USA) ${ }^{66}$}

The Thoratec VAD was first used in 1982 for postcardiotomy support and later in 1984 as a BTT. The device received premarketing approval from the FDA for use as a BTT in 1996. Currently the Thoratec VAD has approval as a BTT and as a BTR in Europe and the USA. The device is an extracorporeal VAD placed in the paracorporeal position that can support the work of the left, right and bilateral ventricles. It consists of four main components: a drive console, inflow cannulae, outflow cannulae and a pump. With the pump in the paracorporeal position, the cannulae enter the patient below the costal margin, crossing the diaphragm and into the mediasternum, where they are connected to the heart. The operation and monitoring of the pump occur through connection to a dual-drive console. Capacity ranges from 1.3 to 7.2 litres/minute with a stroke volume of $65 \mathrm{ml}$ and up to $100 \mathrm{bpm}$ and is suitable for use in small adults and adolescents but not children. It is powered by a large and heavy dual driver but can be ambulatory with a portable driver (9.8-kg battery) which is licensed for use within 2 hours of the hospital. It has three operating modes: asynchronous (to heart) mode with a preset rate and ejection time, volume mode (ejection when VAD filling occurs) and synchronous mode (counterpulsation). The asynchronous mode is recommended for most indications. The paracorporeal position of the Thoratec VAD has advantages and disadvantages. With the major components external to the body, the device can be implanted in smaller patients, although not paediatric patients. Also, its position allows identification of thrombus formation in the pump and the exchange through non-invasive techniques. However, as with many of the mechanical pumps, its position provides the opportunity for infection. Another limitation is the 
restriction on mobility as the patient needs to be accompanied by the large drive console, meaning that the patient must remain within hospital during support. The limitations associated with use within small patients, particularly paediatric cases, and with the limited mobility of the device have resulted in the development of the 'Berlin Heart' in Europe, a portable power and control unit and an implantable device known as the 'Thoratec IVAD'. The Thoratec IVAD is a pneumatically actuated implantable VAD which can support left, right or both ventricles. It weighs $339 \mathrm{~g}$ and has a volume of $252 \mathrm{ml}$. It uses the same internal working components as the VAD. Percutaneous leads connect to an external briefcasesized battery-powered control unit. The IVAD has a smooth contoured, polished titanium housing. It is licensed for trial use in Europe and the USA.

\section{Nippon Zeon LVAD (Nippon Medical Corporation, Japan)}

The Nippon Zeon LVAD is a sac-type, air-driven pump that is placed in the paracorporeal position that is available for adults. It has a stroke volume of $11 \mathrm{ml}$ with inflow in the left or right atrium and operates using mechanical disc valves. Limited information was available on the specification of this device.

\section{Toyobo LVAD (Toyobo Co. Ltd, Japan)}

The Toyobo LVAD is an L-shaped extracorporeal air-driven pneumatic diaphragm-type device using Bjork-Shilley inlet and outlet valves. It has a stroke volume of $70 \mathrm{ml}$ and a maximum output of 7.0 litres/minute. The operating modes include the full-fill to full-empty $(\mathrm{F} / \mathrm{E})$ mode, manual set rate, the ECG or pressure trigger mode and the automatic level control mode using a control drive unit. The device is supported by an automatic backup system.

\section{Displacement: intracorporeal Arrow LionHeart 2000 LVAD (Arrow International, USA)}

The Arrow LionHeart 2000 LVAD is an implantable pulsatile VAD that consists of a pump, a controller and power source. The pump comprises a titanium case with motor, seam-free blood sac, pusher plate and unidirectional inlet/outlet mechanical heart valves. The pump has a stroke volume of $64 \mathrm{ml}$ and a maximum sustained output of 8 litres/minute. It is monitored by the pump controller through Hall effect sensors. A pump controller is implanted, regulating the external power supply, the motor and monitoring. Also, it houses rechargeable batteries that provide temporary power supply of up to 30 minutes when the patient is disconnected from the external supply. The pump controller provides data on the system operation and the opportunity to modify its settings depending on clinical needs. Pumping characteristics are automatically adjusted by a software algorithm through changes to the pump speed. The pump is powered by a transcutaneous energy transmission system (non-invasive), consisting of an external power pack with rechargeable and replaceable batteries, a power transmitter and a primary power coil. The primary power coil is located over an implanted secondary power coil that provides energy to the device. Currently it is licensed for trials in Europe and the USA for long-term support for patients with ESHF. The European trials are nearly complete.

\section{Berlin Heart EXCOR (Mediport Kardiotechnik, Germany) ${ }^{67}$}

The Berlin Heart EXCOR assist device is a pneumatically driven paracorporeal support system that can be used for left, right or both ventricles and is approved for BTT in Europe. It is not available in the USA. The Berlin Heart was first used in 1988 as a BTT and in 1992 became the first commercially available pulsatile device for small children. Although its main use is as a BTT, it has supported patients to cardiac recovery. An extracorporeal VAD, the Berlin Heart is suitable for use in small adults, adolescents and children. The system consists of a paracorporeal air-driven blood pump, cannulae for connection of the pump to the heart and an electropneumatic driving system (stationary or mobile drive units are available). The mobile unit has total running time of 6 hours, weighs $8.7 \mathrm{~kg}$ and is on wheels. The operating regulation is in either synchronous or asynchronous modes with the stationary unit (the synchronous mode is said to prevent a paradox movement of the septum and potentially makes recovery easier). The mobile unit self-regulates by automatically adapting to the patient's requirements within preselected operating parameters. It achieves stroke volumes of between 10 and $80 \mathrm{ml}$ depending on its application and its blood-contacting parts have a heparin coating. As with other extracorporeal devices, the Berlin Heart suffers from certain limitations associated with infection, thrombus and poor device mobility.

\section{Medos HIA-VAD}

The Medos HIA-VAD has external ventricular chambers and is pneumatically operated for either left, right or bilateral ventricles. A range of sizes are available for adults, children and infants and the capacity will change, depending on the 
indication, from 9 to $80 \mathrm{ml}$. Two VADs can be driven independently. A trolley houses the control unit which consists of a touch-screen monitor which ensures easy adjustment of operating parameters. Both fixed-rate and ECG-triggered modes of operation are available. Also on the trolley is the power supply system, which is a compressor unit driven by either the mains or batteries, allowing patient mobility. It is licensed in Europe for BTT and weaning off/recovery.

\section{Terumo Duraheart (Terumo Corporation, Japan)}

This is a magnetically suspended centrifugal pump, with impeller blades, a magnetic bearing and direct current brushless motor. It weighs $400 \mathrm{~g}$ and has a volume of $196 \mathrm{ml}$. It can provide a blood flow of 5-6 litres/minute at pump speeds between 2000 and $3000 \mathrm{rpm}$. It is implanted in the thoracic cavity and used for left ventricular support.

\section{Rotary LVADs}

The new rotary pumps, particularly the axial flow impeller pumps, have been developed following the encouraging results reported from the use of pulsatile devices, with the aim of addressing some of the shortcomings of these devices such as their size and complexity, the difficulties of implantation and adverse events (e.g. perioperative bleeding, infection, thrombosis and device malfunction). These miniaturised implantable pumps provide continuous, nonpulsatile flow using an electromagnetic mechanism consisting of a rotor with impeller blades. These blades are the only moving parts of the device, there are no valves, the devices are biocompatible and there is lower blood damage and no need for anticoagulation with heparin. The control systems and power delivery mechanisms are easily portable and manageable by the patient. The non-pulsatile axial flow pumps currently being developed, such as the Jarvik 2000, MicroMed DeBakey and Nimbus devices, tend to be compact in design, use a single moving part and have lower energy needs. Although these attributes are potentially advantageous, little is known about effects of nonpulsatile flow and concerns have been raised.

The axial flow devices considered in this review are the Jarvik 2000 Heart and the MicroMed DeBakey LVAD. The MicroMed DeBakey device is implanted in a preperitoneal abdominal pocket created by detaching the anterior portion of the left hemidiaphragm, with an inflow cannula to the left ventricle and outflow to the aorta. The Jarvik device is unique in that it is implanted into the body of the left ventricle and therefore has no need for an inflow cannula. The external batteries and controller are carried on a belt. For permanent implants a novel approach is to deliver power via internal electric wires through the left pleural cavity to the apex of the chest and subcutaneously across the neck to the base of the skull. Here, a rigidly fixed percutaneous titanium pedestal transmits fine electrical wires across the skin of the scalp. The combination of immobility and highly vascular scalp skin, which is known to resist infection from experience in cochlear implant technology, is of benefit here. Two further novel rotary blood pumps considered in this review are the HeartQuest, a diagonal pump which is implanted in the abdomen, and the Terumo, a radial pump.

Implantation techniques for smaller LVADs are easier than those for larger devices, with only one body cavity being entered and with shorter times on coronary artery bypass (CAB). The intraventricular position is an advantage as the LVAD stays aligned should there be changes in left ventricular shape during unloading. Malalignment of the device can cause haemolysis, thrombus formation and thromboembolism.

Concerns have been expressed over long-term non-pulsatile blood flow. However, there is some evidence that non-pulsatile blood pumps do provide pulsatility in the circulation as function improves in the off-loaded left ventricle. Complete unloading of the left ventricle leads to a change in the geometry of the right ventricle and an improvement in the right ventricular function which provides pulsatile flow through the lungs so that the preload of the blood pump becomes pulsatile. Pulsatility does not appear to be dependent on pump flow, blood pressure or systemic vascular resistance.

\section{Axial flow devices} Jarvik 2000 (Jarvik Heart Inc., USA) ${ }^{68}$

The Jarvik 2000 is the smallest axial flow device, with an adult version measuring $25 \mathrm{~mm}$ in diameter by $55 \mathrm{~mm}$ in length, weighing $85 \mathrm{~g}$ and with a displacement volume of $25 \mathrm{ml}$, and a smaller paediatric version at one-fifth of the dimensions of the adult device and weighing $18 \mathrm{~g}$ and displacing $5 \mathrm{ml}$. The device is designed for implantation with intraventricular placement. It consists of a rotor housed in a titanium case and supported by ceramic bearings immersed in the bloodstream. The impeller is powered by an electromagnetic field using a small percutaneous cable, with regulation through a brushless direct current motor that determines its speed. The current adult device operates at fixed rate motor 
speeds that are set by the controller at between 8000 and $12,000 \mathrm{rpm}$ with an average capacity of 5 litres/minute. A permanently implantable model is under development that will be responsive to heart rate and which can be set by the patient according to their needs. The Jarvik 2000 has FDA approval for evaluation as BTT in the USA.

\section{MicroMed DeBakey (MicroMed Technology Inc. USA) ${ }^{69}$}

The MicroMed DeBakey VAD is a miniature axial flow pump that has been under development since 1988. The intention was to design a device that overcame the problems associated with other devices, such as haemolysis, thrombosis, noise and heat generation, and also to provide a system that allowed patients to be mobile. The system consists of a pump, a controller and a clinical data acquisition system. The blood pump is a titanium electromagnetically actuated axial flow pump, measuring $30.5 \mathrm{~mm}$ in diameter and $76.2 \mathrm{~mm}$ in length and weighing $93 \mathrm{~g}$. It has a flow capacity of 5 litres/minute at 10,000 rpm and a maximum flow capacity of over 10 litres/minute.The titanium inflow cannula connects the pump to the ventricular apex and a Dacron vascular graft connects the pump to the ascending aorta. A probe placed around the outflow conduit measures blood flow. The wiring of the motor and probe leaves the body through the abdominal wall and is attached to the external controller system. The VAD system aims to be simple to use, although the pump should not be adjusted by the patient. The data acquisition system is used to adjust operating parameters during operation and ITU. A patient home-support system weighing $\sim 4.5 \mathrm{~kg}$ is used for night-time and resting. A controller for mobility weighing $\sim 2.3 \mathrm{~kg}$ can be carried and powered for 6-8 hours. The device has certain limitations, specifically the uncertainties surrounding the long-term effects of non-pulsatile flow on neurovascular and neuroendocrine systems, the problems associated with regurgitant blood flow following pump stoppage and the fact that the pump speed is fixed and does not react to the patient's changing needs. A controller with an adjustable flow rate algorithm that adapts to patients' needs is under development. The MicroMed DeBakey VAD is approved for BTT in the USA for clinical trial use and in Europe for BTT. In the future it is intended for it to be used as a BTR and for chronic support.

\section{Other rotary}

Berlin Incor I (Mediport Kardiotechnik, Germany) The Berlin Heart Incor I is an implantable axial flow pump. It weighs $200 \mathrm{~g}$ and has a diameter of
$30 \mathrm{~mm}$. Its capacity is up to 7 litres/minute and the magnetically suspended impeller is designed to diminish the risks of clots. Percutaneous cannulae run to an external power pack, which weighs $\sim 1500 \mathrm{~g}$. Batteries can give the patients up to 12 hours of mobility. The device received CE mark certification for EU use as BTT and BTR in April 2003. It was intended to seek FDA approval for the Incor device in 2004.

HeartQuest VAD (MedQuest Products Inc., USA) The HeartQuest VAD is a diagonal rotary implantable VAD with percutaneous cannulae. It weighs $600 \mathrm{~g}$ and has a volume of $275 \mathrm{~cm}^{3}$. The rotational speed is $2500 \mathrm{rpm}$, with a flow rate of 10 litres/minute. It uses a magnetic impeller and this, together with a QuestCoat ${ }^{\mathrm{TM}}$ coating, reduces the risk of coagulation. The VAD is able to selfregulate by adjusting to patients' demands and is designed for long-term support.

\section{Assessing the effectiveness of LVADs for ESHF}

Evaluation of the effectiveness of health interventions has increasingly necessitated the assessment of rigorous evidence on their benefits, risks and costs. ${ }^{70}$ The importance of strict evaluation is evident in the regulatory requirements for licensing and for funding of healthcare interventions. Although the RCT is widely recognised as the 'gold standard' for the evaluation of effectiveness, it too has limitations. ${ }^{71-73}$ Conducting RCTs of surgical interventions, such as those involved in implantation of LVADs, requires consideration of certain technical (expertise of clinicians/centres and evolution of procedures), ethical (randomisation in trials) and methodological issues (survival analysis of high-risk interventions). ${ }^{70}$ Importantly, these concerns need to be taken into account when assessing the effectiveness of LVADs for ESHF through systematic review.

With new and complex surgical procedures, the skill of the surgical team and the clinical centre may dramatically affect the outcome of any assessment. If surgeons or clinical centres are selectively assigned to the different procedures, this may explain any apparent difference in outcome. In addition, clinical trials are often conducted by skilled, enthusiastic clinicians within tertiary care centres, which differ considerably from the usual setting in which the service would be delivered. Unsurprisingly, this often limits the 
generalisability of the results. It is rare for studies to identify the expertise of different surgeons or clinical centres, rendering any assessment of the effects on the outcomes of treatment difficult.

Surgical procedures often undergo continued development as they diffuse into practice, leading to two concerns. If clinical trials to assess the surgical procedure are delayed until it has been refined and is relatively stable in terms of development, it may have become common practice and difficult to assess its effectiveness and alter practice. In contrast, if assessed too early in the surgical procedure development, it may provide results that do not represent the procedure that will ultimately be used.

Unfortunately, the nature of surgical interventions often leads to ethical concerns in conducting clinical trials. New interventions tend to be considered as breakthrough treatments for lifethreatening conditions, which may cause difficulties for patients and investigators in accepting the outcome of randomisation. With surgical interventions, patients and clinicians are likely to be aware of the intervention used and will have expectations of the potential outcomes. It may result in people refusing to take part in the trial or to seek treatment outside the trial, causing loss to follow-up or out-of-protocol crossover. It follows that blinding of treating clinicians and patients to such interventions is difficult, if not impossible. A partial solution to preventing observer bias is to use independent observers to assess outcomes. Blinding patients depends on the interventions considered; if they differ considerably, it will be impossible to achieve.

Another methodological concern in the comparison of surgical and medical therapies is the assessment of patient survival. If surgical interventions involve high operative risk but subsequently offer a lower mortality compared with the medical therapy, then survival curves will cross. Analysing the differences in survival curves will be dependent on the analytical methods used and the time period of the analysis. If the analytic method used averages risk over the follow-up period, any changes to the period analysed will have the potential to alter the relative efficacy. Importantly, assessment of effectiveness must take into account not only the differential survival of patients, but also the effects on their QoL. 


\section{Chapter 2}

\section{Research methods for systematic review of clinical and cost-effectiveness of LVADs for ESHF}

$T^{1}$ he $a$ priori methods for systematically reviewing the evidence of clinical and costeffectiveness of LVADs for ESHF and the methods used for developing the economic evaluation are described in the research protocol (Appendix 2). Expert comments on the protocol were obtained from members of the advisory group to the study (see Acknowledgements). Although helpful comments were received relating to the general content of the research protocol, there were none that identified specific problems with the methods proposed. Some changes, additions or points of clarification were made to the methods discussed in the original protocol, as follows:

- It was emphasised that the assessment should identify clearly the different devices that offer left ventricular replacement and those that offer left ventricular assistance and that require some residual heart function. Information on the nature of the different devices included in the systematic review will be presented where available, providing some understanding of their conditions of operation.

- The issue of the long-term effects on organ function of the second-generation devices that use impeller pumps and have a non-pulsatile flow should be assessed. This was recognised as an important issue and, where available, evidence will be presented.

- Aldosterone antagonists as part of the triple therapy and enhanced counterpulsation should be included as a comparator intervention. As the inclusion criteria state that comparators should include LVADs, heart transplantation and/or usual care, this option was not excluded as a comparator.

- The systematic review and economic evaluation should ensure that the methodological quality of the evidence used to assess the clinical and cost-effectiveness is appropriately judged. The research protocol clearly states the methods that will be used to assess methodological quality of included studies and the possible inadequacies of the different measures. As the criteria used are widely recognised as appropriate, it was felt that no further action was needed.
- As part of the assessment of clinical and costeffectiveness of LVADs for ESHF, particularly the implications for developing a service, it would be important to take account of the differences in the nature of the patient groups and the indications for use of the devices. It is likely that most people with ESHF will be elderly and be suffering from co-morbidities which prevent them from receiving a heart transplantation and, as a consequence, from receiving an LVAD as a BTT. In fact, these patients are likely to be candidates for receipt of an LVAD as an LTCS depending upon the guidelines adopted for their use. Younger ESHF patients are more likely to be candidates for heart transplantation and to require an LVAD as a BTT. However, some of these patients will be candidates for LTCS with an LVAD owing to the limited supply of donor hearts. The effects of the different patient groups and the indications for use of LVADs will be assessed in the section in Chapter 5 examining the implications for developing a service, including aspects of budget management and resource management.

The methods outlined in the protocol are summarised below.

\section{Research question}

To assess the clinical and cost-effectiveness of LVADs as a BTT, BTR and potential LTCS for people with ESHF through a systematic review of the evidence and, if necessary, through the development of an economic evaluation.

\section{Search strategy}

Sources of information, search terms and a flowchart outlining the identification of studies for the systematic review and economic evaluation are described in Appendix 3. The electronic search strategy aimed to generate a comprehensive list of studies meeting the inclusion criteria for the systematic review and to provide information for developing the economic evaluation. It included studies published in all languages to reduce the 
potential effects of an English language bias. Reference lists from all publications retrieved were checked for additional publications not identified by the electronic searches. Researchers in the topic area and manufacturers of LVADs included in the systematic review were contacted to check that relevant studies had been identified and to obtain any studies that remain unpublished. In addition, the expert advisory group were asked to assess the comprehensiveness of the search to try to identify any studies not included in the systematic review. It was hoped that these efforts would reduce the effects of publication bias and inaccurate indexing in databases. Searches were updated periodically; the last update was undertaken in October 2003. Although all manufacturers of devices included in the study were contacted for any additional information relevant to the systematic review and economic evaluation, only three submitted any references or data for consideration in the review (Appendix 4). Additional references and information were provided by members of the advisory group and by the key NHS trusts within England and Wales for use in the economic evaluation; these will be discussed further in Chapter 5 .

\section{Inclusion and exclusion criteria for systematic review}

Studies identified in the search strategy were assessed for inclusion in the systematic review of clinical and cost-effectiveness depending on the interventions used, the patient groups, the outcomes assessed and the study design.

\section{Interventions}

Studies that evaluated currently available LVADs used as a BTT, BTR recovery and potential longterm alternative to heart transplantation for people with ESHF were included. LVADs that were in the very early stages of development, no longer available or not used, TAHs, biventricular assist devices (BiVADs), right ventricular assist devices (RVADs) and other blood pumps were excluded. Studies using LVADs for any condition other than left ventricular support were also excluded. Also, studies were excluded from the systematic review of clinical effectiveness if the LVAD was used in conjunction with other interventions where it is impossible to separate out the effects of the different interventions on outcomes or if the study did not clearly identify the LVAD used or included several different devices together and did not report their outcomes separately. However, such studies were considered for inclusion in the systematic review of cost-effectiveness, as it was felt that these studies would contribute
TABLE I4 Devices included in the systematic review of clinical effectiveness

Device
AB-I80 iVAD (implantable)
Abiomed BVS 5000
Arrow LionHeart VAD
Berlin Heart
Berlin Incor I
HeartMate IP (Implanted pneumatic LVAS)
HeartMate VE (vented electric LVAS)
Jarvik 2000
MicroMed DeBakey VAD (Baylor/NASA)
Nippon-Zeon
Novacor (Novacor Medical Corporation/Baxter
Healthcare, Oakland, CA)
Thoratec (implantable VAD, IVAD)
Toyobo

information for the development of the economic evaluation. Importantly, the economic evaluation focused on those LVADs that were shown in the systematic review to be clinically effective for the different indications and relevant to the UK setting.

Searches identified over 35 devices that were considered to be or were used as LVADs (Appendix 1). Advice was sought from the expert advisory group as to the LVADs that should be included in the systematic review and economic evaluation and the reasons as to why devices should be included or excluded. A list of 13 LVADs were included in this systematic review (Table 14).

\section{Participants}

People aged 16 years or older with ESHF and considered suitable for receipt of an LVAD as BTT, BTR or potential long-term alternative to heart transplantation were included in the systematic review. Although certain LVADs have been used to support children with ESHF, it was felt that other factors may affect outcomes and as a consequence these should be examined separately. Patients supported with an LVAD during the perioperative period or when used as an emergency rescue strategy during an operation were also excluded from the systematic review. Again, these patients were thought to represent a different group of patients from the three main indications.

\section{Study designs}

Systematic reviews, RCTs, controlled clinical trials (CCTs), cohort studies, case series, case studies, 
economic evaluations and cost studies were included. Emphasis was placed on studies including an appropriate comparator group, such as people receiving an LVAD with those undergoing heart transplantation, those receiving usual care whilst on the transplantation waiting list or with people receiving a different LVAD. Owing to the scarcity of evidence using an experimental design, natural history studies were sought, as they may provide useful evidence of effectiveness in situations where outcomes are poor and predictable without treatment. ${ }^{74}$ Where there was evidence from different types of study design for a specific LVAD, only those studies with the most rigorous designs were included and data extracted. ${ }^{75}$ Those studies excluded on the rigor of design are listed in Appendix 5. All relevant economic evaluations and cost studies were considered within the systematic review of costeffectiveness and as a source for the economic evaluation.

\section{Outcome measures}

Patient outcomes including survival, functional capacity (e.g. NYHA functional classification, activities of daily living) and QoL were the primary outcome measures considered within the systematic review. Other secondary outcomes included other measures of cardiac function, haemodynamic function, end organ function, adverse events, device-related complications, length of stay, exercise capacity and reoperation. Primary outcome measures were used for judgements regarding the inclusion or exclusion of studies. However, both primary and secondary outcomes were extracted from the included studies and analysed in the systematic review and economic evaluation.

\section{Synthesis of evidence}

Studies were synthesised using a narrative approach through subgroup analysis based on the indication for treatment, type of LVAD and quality of studies. Tables were generated that summarised the results of the included studies, and these are discussed fully in the text. Statistical synthesis by meta-analysis of the data was not appropriate owing to the many differences between the studies in terms of study design, patients or outcomes.

\section{Application of methods for systematic review}

Studies identified by the search strategy were assessed for inclusion through two stages, using criteria described above. For the systematic review of clinical effectiveness, the titles and abstracts of all studies identified by the search strategy were screened independently by two reviewers with any differences in decisions to include or exclude being resolved through discussion or through recourse to a third, independent reviewer. Studies included in the systematic review at this stage were obtained to allow examination of the full text of the study. Any studies on which a decision to include or exclude could not be made at the title and abstract stage owing to a lack of information were also ordered. The full text of the retrieved studies was examined by two independent reviewers to check the decision made. Any disagreements were resolved by discussion or recourse to independent assessment by a third reviewer. These procedures were used to reduce the effects of bias in study selection, which can occur due to the effects of pre-existing opinions of the researcher, and to minimise the risk of errors of judgement. Studies excluded from the review of clinical effectiveness are listed in Appendix 6. The systematic review of cost-effectiveness followed a similar two-stage process differing only in that one reviewer assessed studies at the different stages with a second reviewer checking decisions.

In order to obtain the information needed for the systematic review of clinical effectiveness from the included studies, data were extracted independently by two reviewers using a data extraction form developed a priori (Appendices 7 and 8). The data extraction form noted all of the data items to be extracted in order to minimise bias. The forms for data extraction and guidance for their use were developed and piloted prior to the systematic review, with the aim of familiarising reviewers with the process and reducing subjectivity and error. As with other decisions in the systematic review of clinical effectiveness, any disagreements were resolved through discussion or through recourse to independent assessment by a third reviewer. An historical record of the data extraction processes was maintained as part of the systematic review of clinical effectiveness to allow possible audit of decisions made. Any studies with multiple publications had data extracted on to one form, with any differences between the publications identified and explicitly referenced. If publications from different studies had overlap of their patient cohorts, such as in a multicentre study reporting the results from different combinations of the centres involved, data were extracted from each individual study with a note of potential overlaps on the extraction forms. Where overlaps in the cohorts of different studies were suspected but uncertainty remained, the studies were assessed separately but a note was made of the potential overlap. A similar process 
was followed for the systematic review of costeffectiveness. A form for the extraction of data was developed and piloted a priori (Appendix 9). Data were extracted by one reviewer and checked by a second reviewer, with differences being resolved through discussion or through recourse to a third reviewer.

The methodological quality of the studies included in the systematic review of clinical and cost-effectiveness was assessed using recognised quality assessment tools (Appendices 9, 10 and 11). The assessment of the methodological quality of studies is an essential element of the systematic review of evidence as it allows a judgement to be made as to the rigour of the study and the potential for bias and, as a consequence, the validity of the results. ${ }^{76}$ Judgements about the methodological quality of studies were based on the assessment of individual components of methodological quality rather than relying upon summary scores from particular scales. Although quality scales are appealing owing to their ease of use, the choice of scale may determine the apparent quality of the study and interpretation of the results is often difficult. ${ }^{76}$ It is particularly problematic with the assessment of fast-changing health technologies which encompass evidence from several different levels of the hierarchy. No single quality assessment tool can accurately capture the methodological quality of such different studies into a summary score.

Different quality assessment tools were used for the various types of study design. Systematic reviews and meta-analyses of clinical effectiveness were assessed using criteria specified by NHS Centre for Reviews and Dissemination (CRD) (see Appendix 10), which focuses on the adequacy and appropriateness of inclusion/exclusion criteria, search strategy, assessments of validity, study detail and synthesis of results. The methodological quality of primary studies of clinical effectiveness was assessed using a quality assessment tool developed by Thomas and colleagues ${ }^{77}$ (see Appendix 11). This tool has been recognised as one of the more comprehensive sets of criteria for assessing the quality of different study designs. ${ }^{78}$ The quality assessment tool looks at elements of selection bias, study design, confounders, blinding, data collection methods, withdrawals and drop-outs, intervention integrity and analysis. Although it provides the opportunity to produce summary assessments for the different groups of components and globally, this was not undertaken and reporting of the results of the analysis was based on the components. Interpretation of the quality of primary studies requires caution. With a broad range of study designs included in the assessment of clinical effectiveness, it is inevitable that studies adopting more rigorous experimental designs, such as RCTs or non-randomised clinical trials, are likely to be considered to be of higher methodological quality than observational studies, such as case studies. Using a single quality assessment tool means that a high-quality observational study will not score highly as the tool encompasses components of quality that the observational study does not address by design. With the analysis of methodological quality focused on the opportunities for bias, whether inherent in the design of the study or within the conduct of the study, any high-quality observational studies will be likely to be judged of moderate to weak methodological quality compared with the 'gold standard' RCT. Similarly, RCTs assessing differing types of health technology, such as an operation or device against drug therapy, will not be able to meet all the criteria that are considered to identify a goodquality evaluation (e.g. blinding of patients and clinician). Although such studies may be the most methodologically rigorous given the circumstances, they may be judged as being weak on certain criteria. Although this may be judged to be unfair or inappropriate, it is important to undertake a comparative assessment of the quality and strength of the evidence available for the different devices and indications. The quality criteria used in the assessment of the studies of clinical effectiveness were applied independently by two reviewers, with any disagreements resolved through discussion or through recourse to a third, independent reviewer.

The methodological quality of the economic evaluations included in the systematic review of cost-effectiveness were assessed using a 10-point quality checklist adapted from the methods developed by Drummond and colleagues ${ }^{79}$ (see Appendix 9). As in the systematic review of clinical effectiveness, the components of the quality assessment tool are considered separately rather than as an overall score. The criteria were applied by one reviewer with decisions checked by a second reviewer. Disagreements were resolved through discussion or through recourse to a third reviewer. 


\section{Chapter 3}

\section{Systematic review of the clinical effectiveness of LVADs for people with ESHF}

\section{Introduction}

The clinical effectiveness of LVADS for ESHF was assessed separately for the three indications for treatment of ESHF, specifically as a BTT, BTR and LTCS. For each indication, the quantity and quality of research evidence available for the different LVADs will be discussed and the outcomes of the treatment assessed.

\section{LVADs as a bridge to heart transplantation for ESHF}

\section{Quantity of research}

Sixteen studies assessing the clinical effectiveness of LVADs as a BTT met the inclusion criteria for the systematic review, with 11 studies of firstgeneration devices and five studies of secondgeneration devices (Table 15 and Appendices 12-17). Seven of the studies of first-generation LVADs investigated the use of the HeartMate IP and/or VE devices, with one CCT ${ }^{80}$ three retrospective cohort analytical studies ${ }^{81-83}$ and three cohort studies, either before and after $^{84}$ or compared with a historical control group. ${ }^{85,86}$ Of the six studies comparing the use of the HeartMate LVADs with an alternative intervention, one study compared the HeartMate VE LVAD with the Novacor N100 LVAD, ${ }^{80}$ two studies used inotropic agents as the comparator ${ }^{82,83}$ and three studies did not define the medical care given to the control group. ${ }^{81,85,86}$ The other studies of first-generation LVADs were a cohort analytic study comparing the short- and long-term use of the Novacor LVAD,${ }^{87}$ a case series of the Toyobo LVAD ${ }^{88}$ and two case reports of the Thoratec device. ${ }^{89,90}$ Of the five studies of second-generation LVADs, three were before and after cohort studies of the MicroMed DeBakey LVAD ${ }^{91-93}$ and the other two were case series of the Jarvik 2000 LVAD, one of which was a multicentre case series, with individual centres reporting their patient data at different periods of follow-up for different subgroups. ${ }^{94-100}$

\section{Methodological quality of research First-generation LVADs HeartMate}

The methodological quality and the quality of reporting in the studies assessing the HeartMate LVADs were generally weak (Table 15 and Appendix 12). One study, a CCT comparing the HeartMate VE and Novacor N100 LVADs, had a moderate risk of allocation bias as it used an alternative procedure for allocating people. ${ }^{80}$ The other six studies used a cohort design with nonrandomised allocation of patients to the groups included in the studies, providing the opportunity for allocation bias. The risk of selection bias and confounding was particularly high in five of the studies, ${ }^{81-83,85,86}$ a consequence of either the poor reporting of patient characteristics or the imbalance in the characteristics of the different groups. Although the CCT comparing the HeartMate and Novacor LVADs had a high risk of selection bias owing to the limited information provided on patient selection, there appeared to be no major differences between the groups limiting the possible effects of confounding. ${ }^{80}$ The study by Grady and colleagues ${ }^{84}$ provides information on the selection of participants limiting the opportunity for selection bias. As a before and after cohort study, the effects of confounding were not assessed. ${ }^{84}$ None of the studies adequately described blinding of patients or investigators. Although it is unlikely that patients or investigators would be blinded given the different treatment options under comparison, it would be possible to blind those assessing the outcomes. As a consequence, all seven studies may be affected by measurement bias. Details of the reliability and validity of the methods of data collection used were reported in only one study, ${ }^{84}$ which focused on assessing QoL. The other six studies ${ }^{80-83,85,86}$ provided very limited information on their methods of data collection and as a consequence were rated as being methodologically weak. However, the studies focused on survival, organ function and functional capacity as the main outcomes, which tend to be more objective measures and less prone to bias. Three studies 


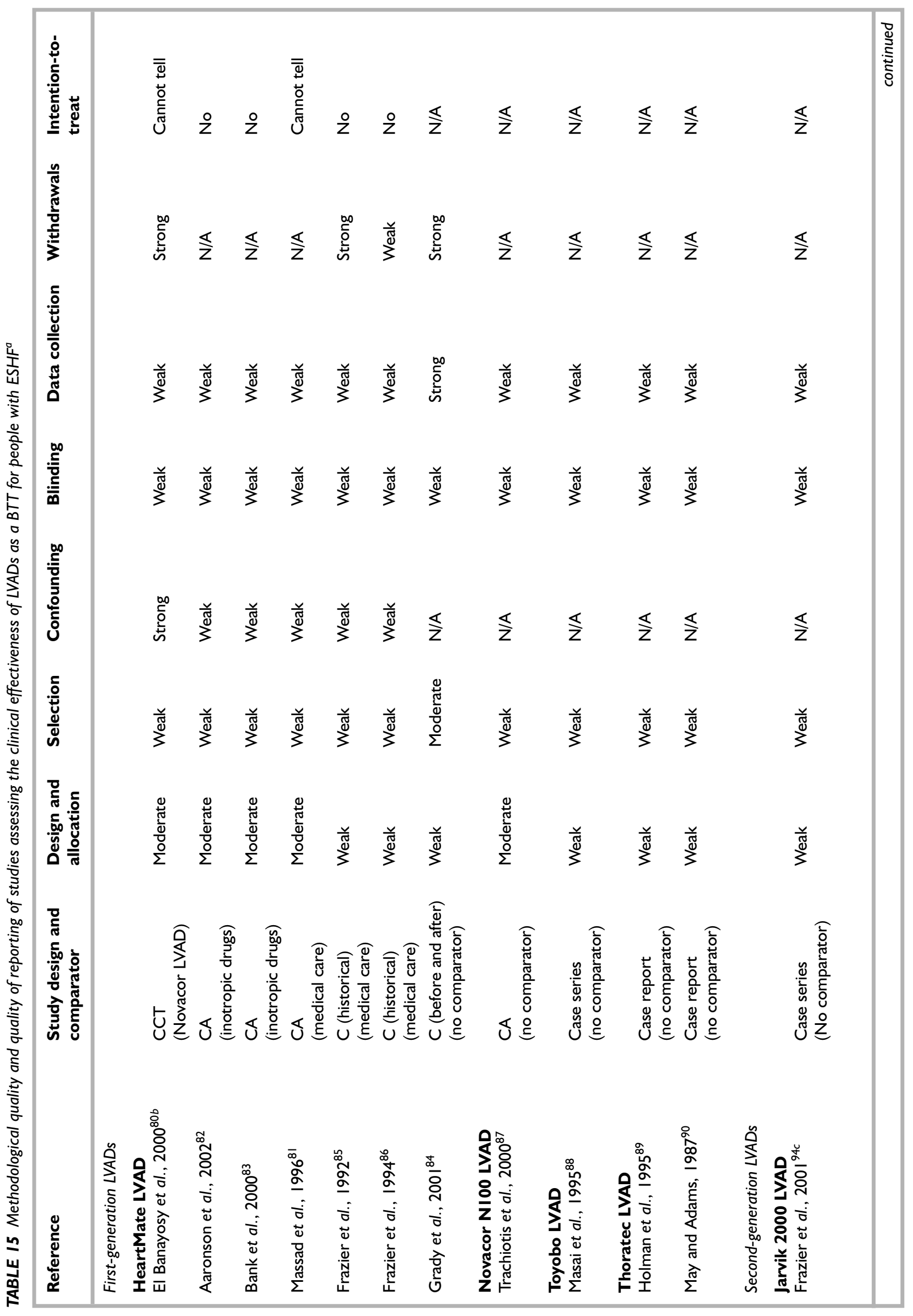




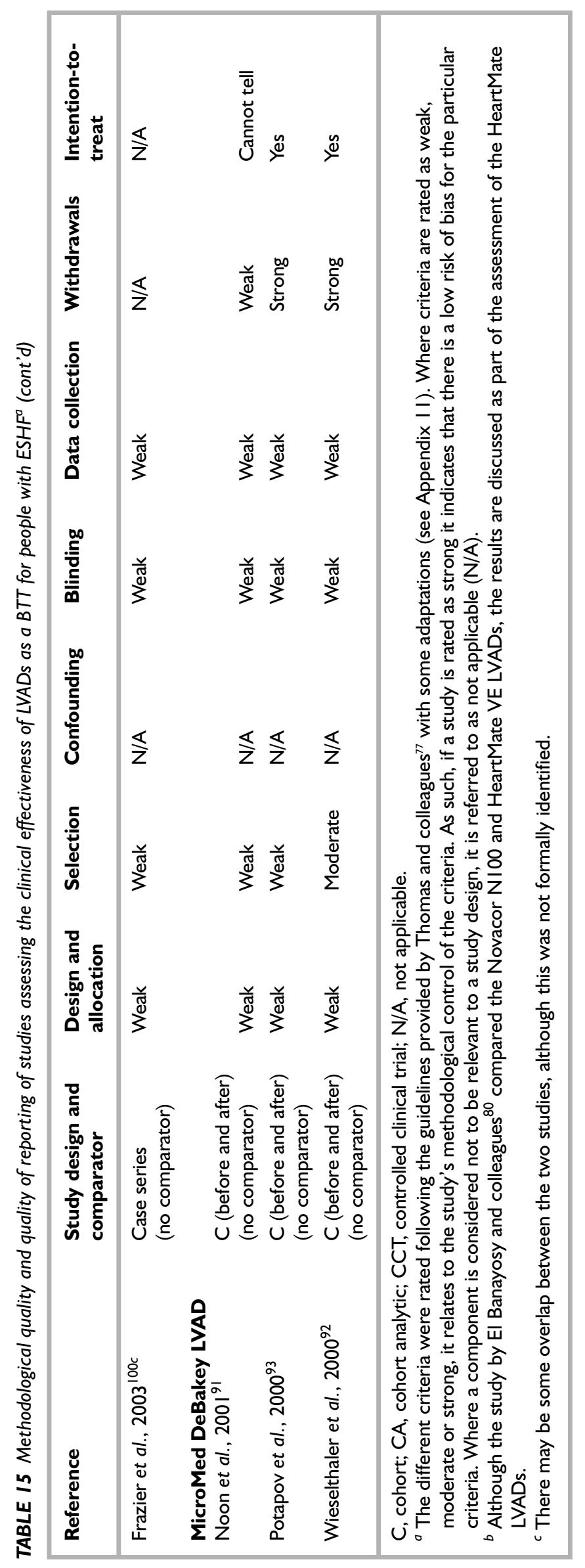


were rated as strong ${ }^{80,84,85}$ and one as weak ${ }^{86}$ on methodological quality of reporting of withdrawals or drop-outs from the different groups. Three studies were not assessed on the criteria for withdrawals as they were considered not applicable for retrospective studies. ${ }^{81-83}$ In addition, none of the studies either reported results using an intention-to-treat analysis ${ }^{82,83,85,86}$ or it was unclear how results were reported, ${ }^{80,81}$ providing the possibility for the studies to be affected by attrition bias. Intention-to-treat analysis was considered not applicable for the study by Grady and colleagues ${ }^{84}$ One retrospective cohort analytic study comparing heart transplant patients supported with an LVAD or usual care reported selected outcomes for an additional group who had an LVAD implanted. ${ }^{82}$ Although the LVAD implanted group included the patients who had had a heart transplant following support from an LVAD, it included patients with an LVAD who did not have a heart transplant. As no information was provided on the characteristics of this group, they were excluded from assessment in the systematic review.

\section{Novacor}

Of the two studies assessing the Novacor LVAD, one compared the LVAD with the HeartMate VE LVAD and the methodological quality is discussed in the section on the HeartMate device above. ${ }^{80}$ The other study, a cohort analytic study assessing the clinical effectiveness of short- and long-term use of the Novacor LVAD, was judged to be of weak methodological quality (see Appendix 13) ${ }^{87}$ Although the study design limited the possible effects of allocation bias, the risk of selection bias was high owing to the poor reporting of the characteristics of the different patient groups. Similarly, there was no description of blinding of the assessment of outcomes with the possibility of the study being affected by measurement bias. Although there was limited discussion of the methods of data collection, outcomes focused on reasonably objective measures of survival and adverse events. The study was not judged on the criteria assessing confounding, withdrawals and intention-to-treat analysis.

\section{Toyobo}

The methodological quality of the case series study assessing the Toyobo LVAD was weak (Appendix 14) ${ }^{88}$ The study provided limited information on the selection of the participants and whether they were representative of the target population or on the methods of data collection and blinding of outcome assessment. As a retrospective case series, the criteria judging confounding, withdrawals and the intention-totreat analysis were considered not relevant.

\section{Thoratec}

The two case reports ${ }^{89,90}$ of the Thoratec device were judged to be of weak methodological quality (Appendix 15). The risk of selection bias was high in both studies, a consequence of the very limited information provided about the patients. Neither study blinded the assessment of outcomes providing the opportunity for ascertainment bias. Although limited information was provided on the tools and methods of data collection, objective outcome measures were assessed limiting the possibility for bias. The criteria for confounding, blinding, withdrawals and the intention-to-treat analyses were not strictly applicable to these types of study design.

\section{Second-generation LVADs Jarvik}

Two case series studies evaluating the Jarvik 2000 LVAD were judged to be methodologically weak, ${ }^{94-100}$ with poor reporting of the selection criteria for participants and the methods of data collection (Appendix 16). Outcome assessment was not blinded, providing the opportunity for ascertainment bias. The criteria judging confounding, withdrawals and intention-to-treat analysis were thought not to be relevant to assess the studies given their study designs.

\section{MicroMed DeBakey}

The methodological quality of the three before and after cohort studies evaluating the MicroMed DeBakey device ${ }^{91-93}$ was judged to be weak (Appendix 17). These studies are subsets of a multicentre study and are therefore likely to include some repeat observations in their publications. Although the authors of these studies were contacted to establish the possible overlap, they were unable to clarify the position. There was a moderate ${ }^{92}$ to high ${ }^{91,93}$ risk of selection bias in the studies as they provided limited information on the selection of patients. None of the studies discuss the blinding of outcome assessment or the validity and reliability of data collection tools and are considered to be methodologically weak. Withdrawals and drop-outs were discussed and patients are analysed on an intention-to-treat basis in two studies, limiting the effects of attrition bias. ${ }^{92,93}$ In the other study, limited information is provided on withdrawals and whether the patients were assessed as intention-to-treat. ${ }^{91}$ The criteria judging confounding were not assessed as they were thought not to be relevant to before and after cohort studies. 


\section{Generalisability}

The generalisability or external validity of the studies was assessed through a comparison of the key characteristics of the patient groups (Table 16). Unfortunately, the lack of information provided in the studies limits any comparisons. The six studies comparing the HeartMate IP and/or VE LVAD with inotropes or usual medical care tended to focus on patients who were male (range 66.7-97\%) with a mean age between 40 and 53 years, classified as NYHA Class IV or in United Network for Organ Sharing (UNOS) status group 1 and with an ischaemic aetiology (29-75\%). The study comparing the HeartMate VE with the Novacor N100 LVAD was mainly focused on males (95\%), with a mean age of 55 years with idiopathic (45-65\%) or ischaemic cardiomyopathy $(30-50 \%) \cdot{ }^{80}$ Similarly, the study of the Novacor LVAD included male patients (100\%) with a mean age of 47-51 years with ischaemic aetiology (40-80\%), whereas the study of the Toyobo LVAD was of males $(100 \%)$ with a mean age of 40 years and idiopathic dilated cardiomyopathy. One study of the Jarvik 2000 LVAD focused on males (66\%) with a mean age of 47 years and suffering from ischaemic aetiology, ${ }^{94}$ whereas in the other case study patient ages ranged from 30 to 70 years, $73 \%$ were male and $50 \%$ suffered from dilated cardiomyopathy and 50\% from ischaemic cardiomyopathy. ${ }^{94-100}$ The patients in the MicroMed DeBakey studies range in age from 33 to 65 years and were predominantly male. The case studies of the Thoratec LVAD each reported male patients. In one ${ }^{90}$ the patient was a 24 -yearold male with a 2-year history of cardiomyopathy. In the second study, ${ }^{89}$ the patient was a 45 -yearold male admitted post-MI.

\section{Assessment of the clinical effectiveness of LVADs as a bridge to heart transplantation \\ Survival \\ First-generation LVADs \\ HeartMate. The CCT by El Banayosy and} colleagues ${ }^{80}$ compared the survival of patients supported with the HeartMate VE LVAD $(n=20)$ with those supported with the Novacor N100 $(n=20)$ through implantation of the LVAD and heart transplantation (Table 17 and Appendix 12). All patients survived implantation of the LVAD devices. El Banayosy and colleagues ${ }^{80}$ reported that $60 \%$ of the patients with the HeartMate VE LVAD and $65 \%$ of the patients with the Novacor LVAD survived to heart transplantation ( $p$ not stated). Although 70\% (14 patients) of the HeartMate and $75 \%$ (15 patients) of the Novacor patients were discharged from hospital to home with the device in place to await transplant, nine patients with the HeartMate and 10 patients with the Novacor had to be readmitted with complications. Three patients with the Novacor LVAD and two patients with the HeartMate LVAD were awaiting transplantation. Four patients with the Novacor LVAD and six patients with the HeartMate LVAD died while on LVAD support.

Two cohort analytic studies ${ }^{82,83}$ assessed the effect on patient survival of the use of HeartMate IP and/or VE LVAD compared with the use of intropic agents (Table 17). The study by Aaronson and colleagues ${ }^{82}$ found a limited difference in survival to heart transplantation for patients receiving a HeartMate LVAD [73\% (9\% still on waiting list), $n$ =66] compared with those patients receiving inotropic agents $(74 \%, n=38)$ ( $p$ not stated). Similarly, actuarial survival to heart transplant was not significantly different $(p=0.2)$ between the patients receiving an LVAD and those receiving inotropic agents at 1 month $(81 \%$ versus $78 \%)$ or at 3 months (81\% versus $64 \%)$. Differences were evident in survival of the groups following heart transplantation. Post-transplantation actuarial survival was significantly $(p=0.007)$ higher for those receiving the HeartMate LVAD compared with those on inotropic agents at 1 year $(98 \%$ versus $74 \%), 3$ years $(95 \%$ versus $65 \%$ ) and 4 years (95\% versus $65 \%$ ). As a consequence, overall actuarial survival from the onset of bridging support was significantly $(p=0.03)$ higher for patients receiving the LVAD $(80 \%$ at 1 year and $77 \%$ at 3 and 4 years) than for those receiving inotropes (56\% at 1 year and $44 \%$ at 3 and 4 years). In reporting the differences in survival, Aaranson and colleagues ${ }^{82}$ noted the disparity between the groups of patients selected for the study and the potential effects on the outcomes assessed. As a consequence, Aaranson and colleagues ${ }^{82}$ undertook a subgroup analysis comparing the patients receiving inotropic agents who were eligible for placement of an LVAD and those considered ineligible owing to the severity of their illness. Analysis of survival to heart transplantation showed that there was no significant $(p=0.18)$ difference between the groups. In addition, Aaronson and colleagues ${ }^{82}$ reported data on a second comparison group $(n=60)$ who were classified as UNOS status 2 patients, that is, patients who are less severely ill than those receiving the LVAD or inotropic agents. When compared with those receiving a HeartMate LVAD, the UNOS status 2 patients had a lower post-transplant actuarial survival rate at 1 year (98\% versus $86 \%$ ), 3 years (95\% versus $77 \%$ ) and 4 years $(95 \%$ versus $77 \%)$, although the difference 


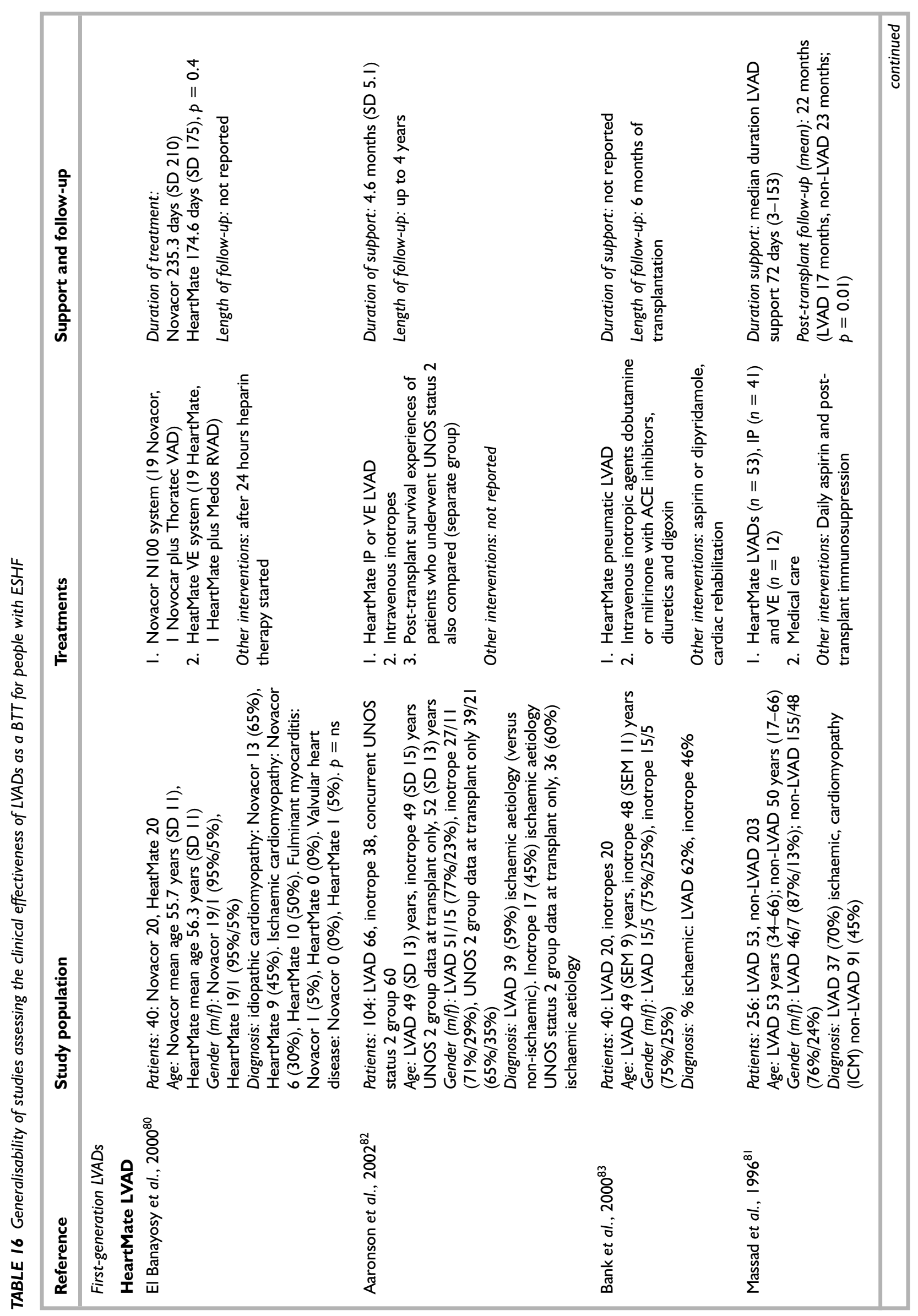




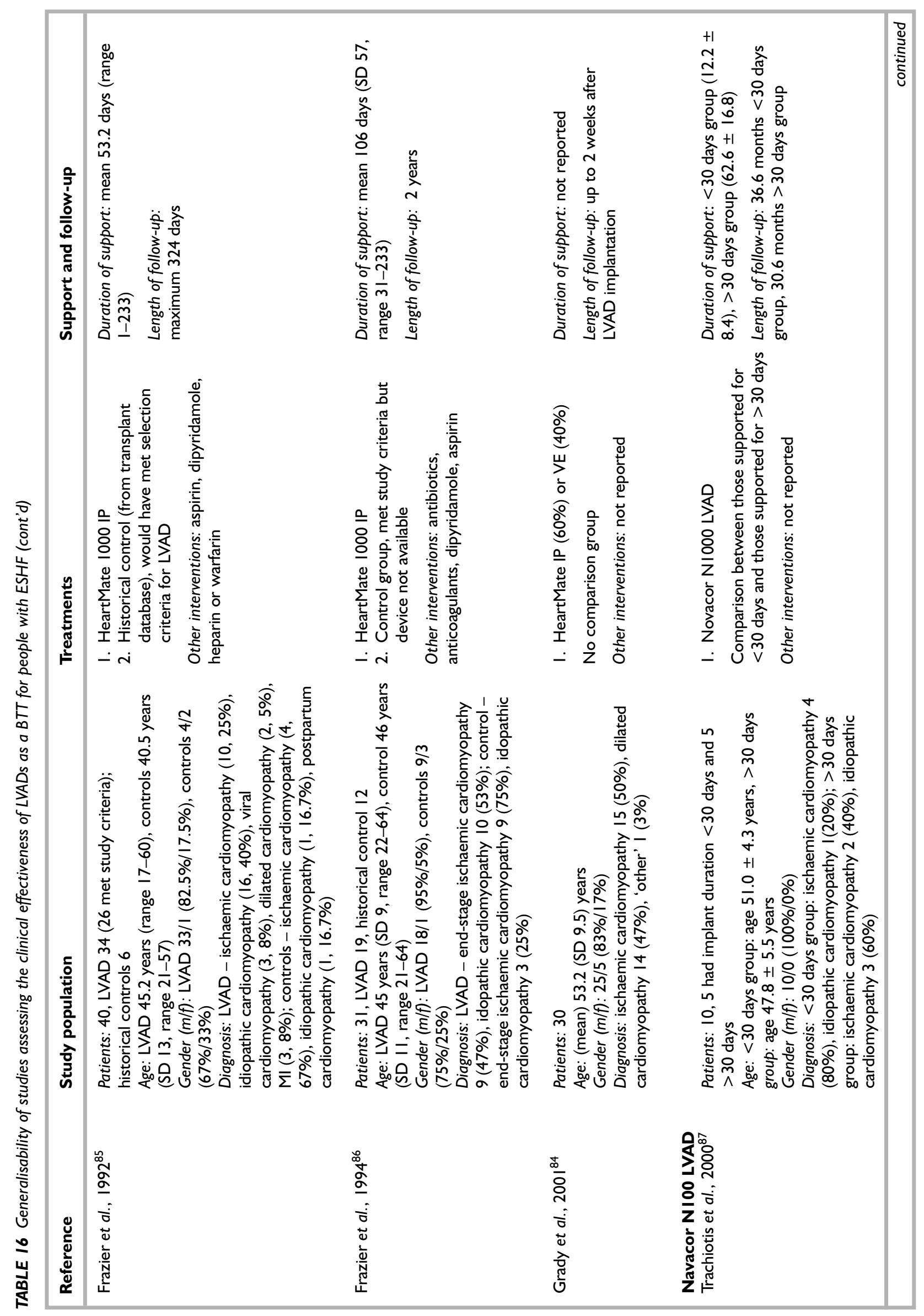




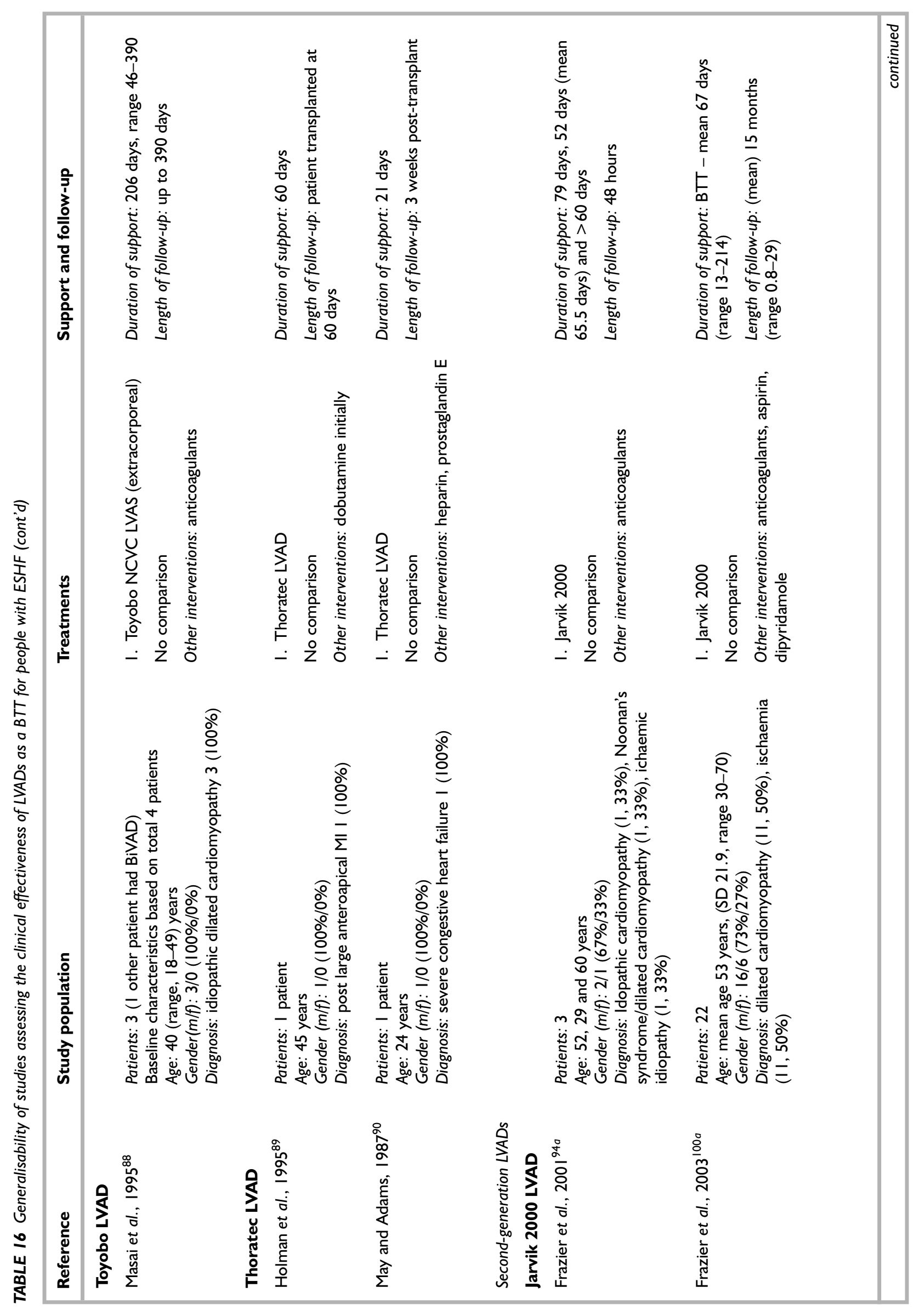




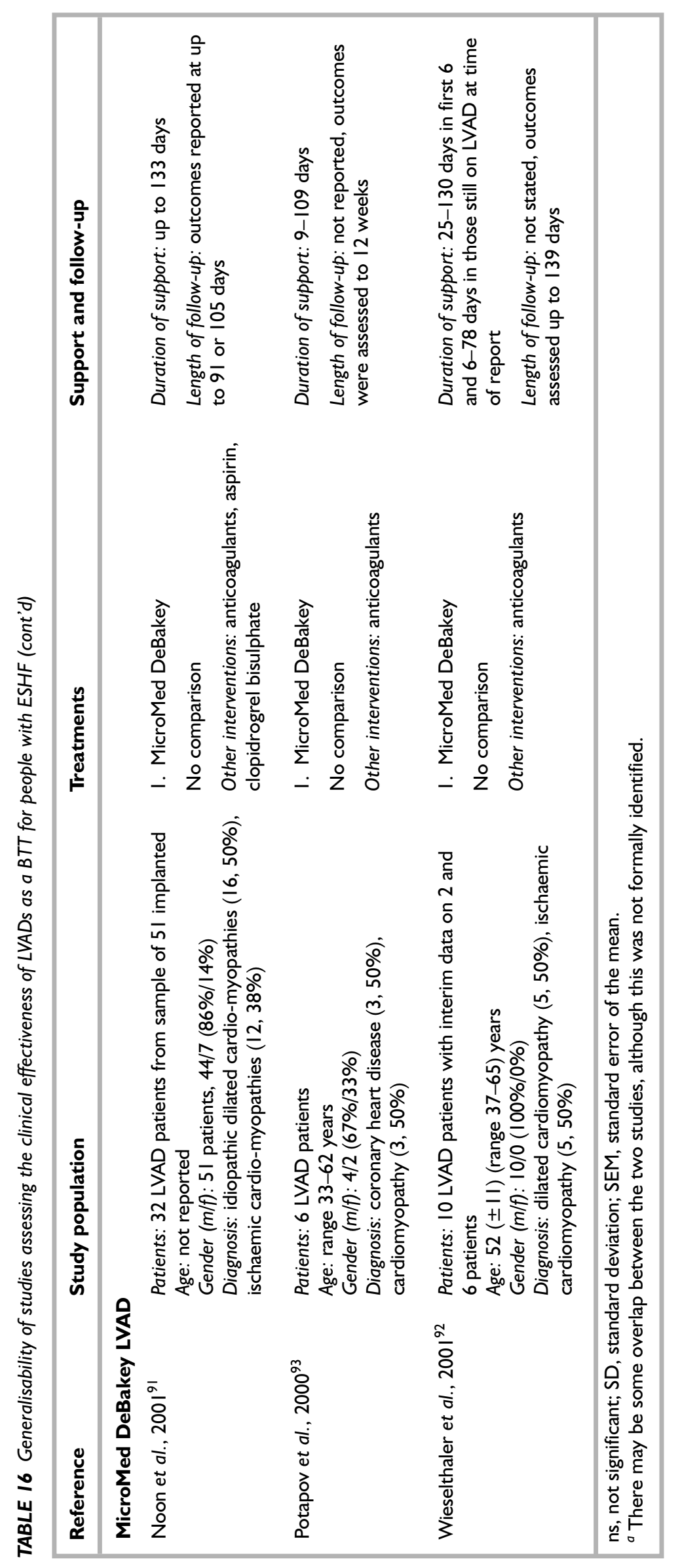


was not significant $(p=0.1)$. In a comparison of the HeartMate IP LVAD $(n=20)$ and inotropic agents $(n=20)$, Bank and colleagues ${ }^{83}$ found a higher but not significant survival at 6 months post-heart transplantation for those receiving an LVAD $(88.9 \%, n=18)$ than for those on inotropes $(73.7 \%, n=19)$ ( $p$ not stated). In addition, patients supported with an LVAD were significantly $(p<0.05)$ more likely to have survived 6 months post-heart transplantation without major complications (55.6\%) compared with those supported by inotropes (15.8\%).

Three studies, one cohort analytic ${ }^{81}$ and two cohort with historic controls, ${ }^{85,86}$ compared patient survival following receipt of a HeartMate LVAD with that from usual care (Table 17 and Appendix 12). In the study by Massad and colleagues, ${ }^{81}$ the comparison of survival to transplant, transplantation rates and post-heart transplant survival for patients who had received a HeartMate LVAD $(n=53)$ with those who were not given an LVAD $(n=203)$ showed no statistically significant difference. Eighty per cent of patients receiving a HeartMate LVAD and $84 \%$ of the patients who were not given an LVAD underwent transplantation $[p=$ not significant (ns)]. Post-operative mortality at 30 days was $3.8 \%$ for those receiving an LVAD and $4.4 \%$ for those who did not have an LVAD $(p=$ ns). Some $91 \%$ of patients receiving an LVAD survived following heart transplantation compared with $86 \%$ of patients who were not given the LVAD after a mean follow-up of 22 months (17 months LVAD group, 23 months non-LVAD group; $p=0.01)$. Similarly, there was no statistically significant difference in actuarial survival at 1-year, $94 \%$ of patients receiving the LVAD and $88 \%$ of patients who were not given the LVAD survived $(p=\mathrm{ns})$. In addition, Massad and colleagues ${ }^{81}$ compared the actuarial survival of patients receiving an LVAD with subgroups of patients who did not receive an LVAD based on their UNOS status [i.e. UNOS status $1(n=126)$ and status $2(n$ $=74)$. At 1 year, patients not receiving an LVAD and who had a UNOS status 1 had an actuarial survival of $91 \%$ compared with $86 \%$ for UNOS status 2 patients and $94 \%$ for patients receiving an LVAD. At 3 years the differences between the groups had decreased, with actuarial survival rates of $85.8 \%$ for UNOS status 1 patients, $82.5 \%$ UNOS status 2 patients and $84.9 \%$ for the patients receiving LVADs ( $p$ not stated). Although Massad and colleagues ${ }^{81}$ found no statistically significant difference in measures of survival between the different treatment groups, the poor methodological quality of the study suggest that the results should be interpreted with caution.
Significant differences in the two groups' baseline characteristics, particularly their UNOS status and underlying diagnosis, may affect the survival rates (Table 17 and Appendix 12).

Frazier and colleagues ${ }^{85}$ retrospectively studied patients with HeartMate IP LVADs $(n=34)$ and compared these with historical controls who received usual care $(n=6)$. In the LVAD group, eight patients did not meet the study inclusion criteria but received an LVAD and were included in the analysis of patient survival (Table 17 and Appendix 12). Sixty-four per cent of all patients who received an LVAD survived to heart transplantation (excludes three patients awaiting transplantation) compared with $50 \%$ of patients receiving usual care. Of the 20 LVAD patients transplanted, 17 patients met the eligibility criteria for receipt of an LVAD and three did not. Three patients with an LVAD were still waiting for heart transplantation at the end of the study. Survival of patients for over 60 days post-heart transplant differed significantly between the two groups $(p<0.05)$, with $52 \%(n=31)$ of transplanted patients with an LVAD surviving compared with $17 \%(n=6)$ of those on usual care. Among the 23 patients who met the criteria for and received an LVAD, 65\% survived for $>60$ days after receiving their heart transplant compared with $13 \%$ of the eight patients not meeting the criteria. The survival rate of the LVAD patients was significantly greater than that for the patients receiving usual care $(p<0.05)$.

In another cohort study comparing HeartMate IP LVAD patients $(n=19)$ with historical medical controls $(n=12)$, Frazier and colleagues ${ }^{86}$ reported higher survival rates among patients with the LVADs (Table 17 and Appendix 12). Eightyfour per cent of patients with an LVAD underwent heart transplantation with $11 \%$ of patients dying during support. In contrast, $25 \%$ of patients receiving usual medical care underwent heart transplantation, with the remaining $75 \%$ dying while awaiting transplant. None of the patients receiving usual medical care survived transplantation. One patient with an LVAD remained on the heart transplant waiting list. Actuarial survival rates at one and 2 years posttransplant for patients receiving an LVAD were $100 \%(n=16)$ compared with $0 \%(n=3)$ for the patients receiving usual medical care $(p<0.05)$.

Novacor. One retrospective cohort analytic study compared survival of patients supported with Novacor N100 LVAD for $<30$ days $(n=5)$ with those supported for $>30$ days $(n=5)$ (Table 17 
TABLE I7 Summary of evidence of patient survival following treatment of patients with ESHF as a BTT

\begin{tabular}{|c|c|c|c|c|}
\hline Study & Outcomes & Intervention & Comparison & $p$-Value \\
\hline \multicolumn{5}{|l|}{ First-generation LVADs } \\
\hline \multirow[t]{2}{*}{$\begin{array}{l}\text { HeartMate LVAD } \\
\text { El Banayosy et al., } \\
2000^{80}\end{array}$} & Survival to transplantation (\%) & $\begin{array}{l}\text { HeartMate } \\
12 / 20(65)\end{array}$ & $\begin{array}{l}\text { Novacor } \\
13 / 20(60)\end{array}$ & Not stated \\
\hline & \multicolumn{4}{|c|}{3 Novacor group and 2 HeartMate group are awaiting transplantation } \\
\hline \multirow[t]{6}{*}{ Aaronson et al., $2002^{82}$} & Survival to transplant (\%) & $\begin{array}{l}\text { HeartMate } \\
48 / 66(73)\end{array}$ & $\begin{array}{l}\text { Inotropic agents } \\
28 / 38(74)\end{array}$ & Not stated \\
\hline & $\begin{array}{l}\text { Actuarial survival to transplant } \\
(\% \pm S D)\end{array}$ & $\begin{array}{l}\text { I month: } 8 \text { I (SD 5) } \\
3 \text { months: } 8 \text { I (SD 5) } \\
\text { Median: } 2.9 \text { months }\end{array}$ & $\begin{array}{l}\text { I month: } 78 \text { (SD 8) } \\
3 \text { months } 64 \text { (SD II) } \\
\text { Median: } 2.9 \text { months }\end{array}$ & 0.2 \\
\hline & No. alive post-transplantation (\%) & $\begin{array}{l}0 \text { days: } 48(78) \\
30 \text { days: } 47(7 \mathrm{I}) \\
\text { I year: } 3 \text { I }(47) \\
3 \text { years: } 9(14)\end{array}$ & $\begin{array}{l}0 \text { days: } 28(74) \\
30 \text { days: } 24(63) \\
\text { I year: } 17(45) \\
3 \text { years: } 6(16)\end{array}$ & Not stated \\
\hline & $\begin{array}{l}\text { Post-transplantation actuarial } \\
\text { survival }(\% \pm S D)\end{array}$ & $\begin{array}{l}\text { I year: } 98(\text { SD } 2) \\
3 \text { years: } 95(\text { SD } 4) \\
4 \text { years: } 95(\text { SD } 4)\end{array}$ & $\begin{array}{l}\text { Inotrope: } \\
\text { I year: } 74 \text { (SD 9) } \\
3 \text { years: } 65 \text { (SD 10) } \\
4 \text { years: } 65 \text { (SD I0) }\end{array}$ & $\begin{array}{l}\text { LVAD vs } \\
\text { inotrope, } \\
0.007\end{array}$ \\
\hline & & & $\begin{array}{l}\text { UNOS status 2: } \\
\text { I year: } 86 \text { (SD 4) } \\
3 \text { years: } 77 \text { (SD 7) } \\
4 \text { years: } 77 \text { (SD 7) }\end{array}$ & $\begin{array}{l}\text { LVAD vs } \\
\text { Unos } \\
\text { status } 2,0.1\end{array}$ \\
\hline & $\begin{array}{l}\text { Overall actuarial survival } \\
(\% \pm S D)\end{array}$ & $\begin{array}{l}\text { I year: } 80(\text { SD 5) } \\
3 \text { years: } 77(\text { SD 6) } \\
4 \text { years: } 77(\text { SD 6) }\end{array}$ & $\begin{array}{l}1 \text { year: } 56(\text { SD } 8) \\
3 \text { years: } 44(\text { SD 9) } \\
4 \text { years: } 44 \text { (SD 9) }\end{array}$ & 0.03 \\
\hline \multirow[t]{3}{*}{ Bank et al., $2000^{83}$} & & HeartMate & Inotropic agents & \\
\hline & $\begin{array}{l}\text { Survival } 6 \text { months post-transplant } \\
\text { (\%) }(n=18 \text { LVAD, } 19 \text { inotrope })\end{array}$ & 16/18 (88.9) & $14 / 19(73.7)$ & Not stated \\
\hline & $\begin{array}{l}\text { Survival to } 6 \text { months without major } \\
\text { complications post-transplant (\%) }\end{array}$ & $10 / 18(55.6)$ & $3 / 19(15.8)$ & $<0.05$ \\
\hline \multirow[t]{5}{*}{ Massad et al., 1996 ${ }^{81}$} & & HeartMate & Medical care & \\
\hline & Transplant rate (\%) & $42 / 53(80)$ & I70/203 (84) & ns \\
\hline & Survivors (\%) & $48 / 53(91)$ & I74/203 (86) & ns \\
\hline & $\begin{array}{l}\text { Actuarial survival at I year } \\
\text { (Kaplan-Meier) }\end{array}$ & $94 \%$ & $\begin{array}{l}88 \% \text { (all non-LVAD) } \\
\text { UNOS I: } 91 \% \\
\text { UNOS 2: } 86 \%\end{array}$ & ns \\
\hline & 36-month survival (Kaplan-Meier) & $84.9 \%$ & $\begin{array}{l}\text { UNOS I: } 85.8 \% \\
\text { UNOS } 2: 82.5 \%\end{array}$ & \\
\hline \multirow[t]{3}{*}{ Frazier et al., $1992^{85}$} & & HeartMate & Medical care & \\
\hline & $\begin{array}{l}\text { Received transplant }(\%) \\
\text { ( } 3 \text { remained on LVAD) }\end{array}$ & $\begin{array}{l}\text { Total with LVAD: } \\
\text { 20/3 I (64) } \\
\text { Subset with LVAD } \\
\text { who meet study } \\
\text { criteria: } 17 / 23 \text { (74) }\end{array}$ & $3 / 6(50)$ & Not stated \\
\hline & Survival $>60$ days $(\%)$ & $\begin{array}{l}\text { Total with LVAD: } \\
\text { 16/3 I (52) } \\
\text { Subset with LVAD } \\
\text { who meet study } \\
\text { criteria: } 15 / 23(65)\end{array}$ & $1 / 6(17)$ & $\begin{array}{l}\text { Survival rate } \\
\text { of LVAD } \\
\text { group } \\
\text { greater than } \\
\text { control, } \\
<0.05\end{array}$ \\
\hline
\end{tabular}


TABLE I7 Summary of evidence of patient survival following treatment of patients with ESHF as a BTT (cont'd)

\begin{tabular}{|c|c|c|c|c|}
\hline Study & Outcomes & Intervention & Comparison & p-Value \\
\hline \multirow[t]{3}{*}{ Frazier et al., $1994^{86}$} & & HeartMate & Medical care & \\
\hline & Successful transplantation (\%) & $16 / 19(84)$ & $0 / 12(0)$ & \\
\hline & Actuarial survival land 2 years & $\begin{array}{l}16 \text { with successful } \\
\text { transplant: } 100 \%\end{array}$ & $0 / 3(0)$ & $<0.05$ \\
\hline Grady et al., $2001^{84}$ & Survival not assessed & & & \\
\hline \multicolumn{5}{|l|}{ Novacor NIO0 LVAD } \\
\hline \multirow[t]{5}{*}{ Trachiotis et al., $2000^{87}$} & & LVAD $<30$ days & LVAD $>30$ days & \\
\hline & $\begin{array}{l}\text { Survival post-transplant or } \\
\text { explant } 30 \text { days (\%) }\end{array}$ & 100 & 100 & Not stated \\
\hline & $\begin{array}{l}\text { Survival post-transplant or explant } \\
\text { I year }(\%)\end{array}$ & 100 & 80 & Not stated \\
\hline & $\begin{array}{l}\text { Survival post-transplant or explant } \\
2 \text { year }(\%)\end{array}$ & 100 & Not reported & Not stated \\
\hline & $\begin{array}{l}\text { Survival post-transplant or explant } \\
3 \text { year }(\%)\end{array}$ & 60 & 60 & Not stated \\
\hline \multicolumn{5}{|l|}{ Toyobo LVAD } \\
\hline Masai et al., $1995^{88}$ & Survival & $\begin{array}{l}\text { Patient I supported } \\
\text { for I I } 9 \text { days then } \\
\text { transplanted. } \\
\text { Patient } 2 \text { still } \\
\text { supported at } 390 \text { days. } \\
\text { Patient } 3 \text { supported } \\
\text { for } 64 \text { days then died }\end{array}$ & $\begin{array}{l}\text { No comparison } \\
\text {. }\end{array}$ & Not stated \\
\hline \multicolumn{5}{|l|}{ Thoratec LVADs } \\
\hline Holman et al., $1995^{89}$ & Survival & $\begin{array}{l}\text { Patient survived for } \\
60 \text { days and then } \\
\text { transplanted }\end{array}$ & No comparison & Not stated \\
\hline May and Adams, $1987^{90}$ & Survival & $\begin{array}{l}\text { Patient survived for } \\
3 \text { weeks post- } \\
\text { transplant and was } \\
\text { discharged home }\end{array}$ & No comparison & Not stated \\
\hline \multicolumn{5}{|l|}{ Second-generation LVADs } \\
\hline \multicolumn{5}{|l|}{ Jarvik 2000 LVADs } \\
\hline Frazier et al., $2001^{94 a}$ & Successful transplant (\%) & $2 / 3(67)$ & No comparison & Not stated \\
\hline Frazier et al., $2003^{100 a}$ & Survival & $\begin{array}{l}\text { Of } 22 \text { patients, } \\
13 \text { underwent heart } \\
\text { transplant; } 7 \text { died } \\
\text { awaiting transplant, } \\
2 \text { ongoing at } 92 \text { and } \\
\text { I05 days post-implant }\end{array}$ & No comparison & Not stated \\
\hline \multicolumn{5}{|c|}{ MicroMed DeBakey LVADs } \\
\hline \multirow[t]{3}{*}{ Noon et al., $200 \mathrm{I}^{91}$} & Probability of survival at 30 days & $81 \%$ & No comparison & Not stated \\
\hline & Number transplanted (\%) & II of 32 patients (34) & No comparison & Not stated \\
\hline & Deaths on support (\%) & 10 of 32 patients $(3 \mathrm{I})$ & No comparison & Not stated \\
\hline
\end{tabular}


TABLE I 7 Summary of evidence of patient survival following treatment of patients with ESHF as a BTT (cont'd)

\begin{tabular}{|c|c|c|c|c|}
\hline Study & Outcomes & Intervention & Comparison & $p$-Value \\
\hline \multirow[t]{2}{*}{ Wieselthaler et al., 2001 ${ }^{92}$} & Survival (\%) & $\begin{array}{l}8(80) \text { survived; } 4 \\
(40) \text { transplanted; } \\
4(40) \text { awaiting } \\
\text { transplant; } 2(20) \\
\text { died }\end{array}$ & No comparison & Not stated \\
\hline & Deaths on support (\%) & $\begin{array}{l}2(20) \text { patients } \\
\text { (I patient died from } \\
\text { MOF after } 25 \text { days; } \\
\text { I patient died infectious } \\
\text { complications leading } \\
\text { to MOF I } 42 \text { days } \\
\text { post-operation) }\end{array}$ & $\begin{array}{l}\text { No comparison } \\
\text { Is }\end{array}$ & Not stated \\
\hline \multirow[t]{2}{*}{ Potapov et al., $2000^{93}$} & Number transplanted (\%) & $2(34)$ & No comparison & Not stated \\
\hline & Deaths on support (\%) & $\mathrm{I}(\mathrm{I})$ & No comparison & Not stated \\
\hline
\end{tabular}

and Appendix 13). ${ }^{87}$ Survival at 30 days post-heart transplant or explant of the LVAD (one patient was explanted) was $100 \%$ in both groups. At 1 year post-transplant, survival in those supported for $<30$ days was still $100 \%$, but had declined to $80 \%$ in the group who had been supported for $>30$ days. After 3 years, however, survival in both groups was $60 \%$. No statistical data analysis was reported in the study. A CCT comparing the Novacor N100 with the HeartMate VE LVAD reported survival to transplant; results are presented in the section discussing the HeartMate LVADs.

Toyobo. One case series study reported survival as the duration of support with the Toyobo LVAD for three patients (Table 17 and Appendix 14). ${ }^{88}$ One patient was supported for 119 days then transplanted, one was still supported and awaiting a transplant at 390 days and the final patient was supported for 64 days but then died.

Thoratec. Two case reports of the Thoratec LVAD reported survival data (Table 17 and Appendix 15). ${ }^{89,90}$ In one a 45 -year-old male patient survived for 60 days and was then transplanted. ${ }^{89}$ In the second case report, ${ }^{90}$ a 24 -year-old male was supported for 21 days and then transplanted; he was discharged home 3 weeks after transplantation.

\section{Second-generation LVADs}

Jarvik. Two case series of the Jarvik 2000 LVAD reported survival to transplantation (Table 17 and Appendix 16). ${ }^{94-100}$ In a case series ${ }^{94}$ of three patients, a successful heart transplant rate of $67 \%$ was reported with one patient still awaiting transplant. A multicentre case series study, which also assessed patients supported long term with LVAD (see the section 'LVADs as a long-term chronic support' p. 58), reported survival to heart transplantation for 22 patients with the indication of BTT. ${ }^{95-100}$ Of these, 13 patients underwent heart transplant, seven patients had died and two patients continued to be supported by the device.

MicroMed DeBakey. A multicentre before and after cohort study of the MicroMed DeBakey device has been undertaken in the USA and Europe (Table 17 and Appendix 17). Some of the centres involved in the study have published their results separately. ${ }^{91-93}$ One paper reports data from the first 32 of the 51 patients studied to date and may include patients reported by the other centres. ${ }^{91}$ Probability of survival in the 32 patients at 30 days was $81 \%$. Of these 32 patients, 11 received a heart transplant and 10 died. In the reports from the Austrian centre, ${ }^{92,101-103}$ eight of the 10 patients included survived to follow-up; four of these were transplanted and four were awaiting transplantation. In the study by the German centre, ${ }^{93}$ five of the six patients included survived to follow-up. Two of these received a heart transplant.

\section{Functional status}

\section{First-generation LVADs}

HeartMate. Two cohort studies with historical controls report data on functional status of patients using the NYHA functional classification (Table 18 and Appendix 12). ${ }^{85,86}$ Frazier and 
TABLE I8 Summary of evidence of patients' functional status following treatment of patients with ESHF as a BTT

\begin{tabular}{|c|c|c|c|c|}
\hline Study & Outcomes & Intervention & Comparison & $p$-Value \\
\hline \multicolumn{5}{|l|}{ First-generation LVADs } \\
\hline \multirow[t]{3}{*}{$\begin{array}{l}\text { HeartMate LVAD } \\
\text { Frazier et al., } 1992^{85}\end{array}$} & NYHA functional class & HeartMate LVAD & Usual medical care & \\
\hline & $\begin{array}{l}\text { LVAD implantation/time of meeting } \\
\text { study criteria (for controls) (\%) }\end{array}$ & $\begin{array}{l}34 / 34(100) \text { in } \\
\text { Class IV }\end{array}$ & $\begin{array}{l}\text { 5/6 (83) Class IV } \\
\text { I/6 (17) Class III }\end{array}$ & Not stated \\
\hline & $\begin{array}{l}60 \text { days after transplantation } \\
\text { (surviving patients) }\end{array}$ & $\begin{array}{l}\text { I5/16 (94) in Class I } \\
\text { I/16 (6) in Class II }\end{array}$ & I/I (I00) Class IV & Not stated \\
\hline \multirow[t]{3}{*}{ Frazier et al., $1994^{86}$} & NYHA functional class & HeartMate LVAD & Usual medical care & \\
\hline & Implantation & All Class IV $(n=19)$ & $\begin{array}{l}\text { Not reported for } \\
\text { comparator }\end{array}$ & $N / A$ \\
\hline & Transplant (\%) & $\begin{array}{l}\text { I5/I6 (94) Class I } \\
\text { (I patient unable to } \\
\text { ambulate owing to } \\
\text { poor position of } \\
\text { pneumatic drive line) }\end{array}$ & $\begin{array}{l}\text { Not reported for } \\
\text { comparator }\end{array}$ & N/A \\
\hline \multicolumn{5}{|l|}{ Second-generation LVADs } \\
\hline \multirow{4}{*}{$\begin{array}{l}\text { Jarvik } 2000 \text { LVAD } \\
\text { Westaby et al., } 2002^{95}\end{array}$} & & & & \\
\hline & NYHA functional class & Jarvik 2000 & No comparator & \\
\hline & Baseline $(n=10)$ & 10/10 in Class IV & & N/A \\
\hline & Post-implantation of LVAD $(n=10)$ & $\begin{array}{l}7 / 10 \text { in Class I } \\
3 / 10 \text { died }\end{array}$ & & $N / A$ \\
\hline
\end{tabular}

colleagues $^{85}$ compared the change in the NYHA functional class for patients receiving an LVAD with those on usual medical care. Prior to treatment $100 \%$ of patients in the LVAD group $(n=34)$ were in NYHA Class IV, whereas $83 \%$ of patients receiving usual medical care $(n=6)$ were in Class IV and 17\% were in Class III. Of the 16 patients with an LVAD surviving 60 days post-heart transplantation, $94 \%$ were in Class I and $6 \%$ in Class II. In contrast, the one patient in the control group who survived heart transplantation was in Class IV ( $p$ not reported). In another study, Frazier and colleagues ${ }^{86}$ reported that all patients receiving an LVAD were in NYHA Class IV prior to implantation $(n=19)$, with $94 \%$ in NYHA Class I following heart transplantation (although one patient was unable to ambulate owing to the poor position of the pneumatic drive line) ( $p$ not reported). Change in NYHA classification was not reported by Frazier and colleagues ${ }^{86}$ for the historical control group who received usual care.

\section{Second-generation LVADs}

Jarvik. Only one study, a subset of patients in a case series of the Jarvik 2000 LVAD, ${ }^{95}$ reported changes in the functional status of patients (Table 18 and Appendix 16). The 10 patients prior to implantation of the Jarvik 2000 LVAD. Post-implantation of the device, seven patients improved to NYHA Class I and three patients died.

\section{Quality of life}

First-generation LVADs

HeartMate. One cohort study by Grady and colleagues $^{84}$ reported the QoL of 30 patients with the HeartMate LVAD before and 1-2 weeks after implantation on a range of indices (see Table 19 and Appendix 12). On the Quality of Life Index (score from $0.00=$ very dissatisfied to $1.00=$ very satisfied) the mean (SD) total QoL improved significantly $(p=0.037)$ from $0.66(0.14)$ before implantation to $0.73(0.13) 1-2$ weeks after implantation. There were significant improvements in satisfaction on the subcategories of significant others (before 0.84, after 0.90; $p=0.002$ ) and health functioning (before 0.51 , after $0.66 ; p=0.001$ ), but significant worsening of satisfaction in the socio-economic subcategory (before 0.72 , after $0.50 ; p<0.0001$ ). In contrast, there was no change in the psychological subcategory (before 0.64; after $0.64 ; p=\mathrm{ns}$ ). Patients were generally more satisfied than dissatisfied with their lives before and after surgery as all mean scores were found to be $\geq 0.50$. Similarly on the Heart Failure Symptom Checklist 
TABLE 19 Summary of evidence of patients' QoL following treatment of patients with ESHF as a BTT

\begin{tabular}{|c|c|c|c|c|}
\hline Study & Outcomes & Baseline & Follow-up & p-Value \\
\hline \multicolumn{5}{|l|}{ First-generation LVADs } \\
\hline \multirow[t]{39}{*}{$\begin{array}{l}\text { HeartMate LVADs } \\
\text { Grady et al., } 2001^{84}\end{array}$} & & Before implantation & $\begin{array}{l}\text { I-2 weeks after } \\
\text { implantation }\end{array}$ & \\
\hline & QOL Index ( 0.00 very dissatisfied, & $.00=$ very satisfied $)$ & ean $\pm S D)$ & \\
\hline & Significant others & $0.84(0.10)$ & $0.90(0.08)$ & 0.002 \\
\hline & Socio-economic & $0.72(0.21)$ & $0.50(0.25)$ & $<0.0001$ \\
\hline & Psychological & $0.64(0.22)$ & $0.64(0.24)$ & ns \\
\hline & Health functioning & $0.51(0.18)$ & $0.66(0.17)$ & 0.001 \\
\hline & Total score & $0.66(0.14)$ & $0.73(0.13)$ & 0.037 \\
\hline & Rating Question Form (mean \pm & & & \\
\hline & $\begin{array}{l}\text { Stress level }(I=\text { no stress, } \\
10=\text { very much stress })\end{array}$ & $6.2(2.5)$ & $4.9(2.9)$ & ns \\
\hline & $\begin{array}{l}\text { Coping ability }(I=\text { very poorly, } \\
10=\text { very well })\end{array}$ & $7.7(2.0)$ & $6.6(2.6)$ & $0.026(\mathrm{~ns})^{a}$ \\
\hline & $\begin{array}{l}\text { Health }(I=\text { very poor, } \\
I 0=\text { very good })\end{array}$ & $4.0(3.1)$ & $6.2(2.4)$ & $0.012(\mathrm{~ns})^{a}$ \\
\hline & $\begin{array}{l}\text { QoL }(I=\text { very poor, } \\
10=\text { very good })\end{array}$ & $3.5(2.5)$ & $5.9(2.7)$ & 0.002 \\
\hline & $\begin{array}{l}\text { How well will do/doing after } \\
\text { LVAD (I = very poorly, } \\
\text { IO = very well })\end{array}$ & $8.7(1.3)$ & $7.1(2.1)$ & 0.001 \\
\hline & $\begin{array}{l}\text { How well will do/doing after HT } \\
\text { (I= very poorly, } I 0=\text { very well) }\end{array}$ & $9.3(0.9)$ & $9.3(1.1)$ & ns \\
\hline & $\begin{array}{l}\text { Heart Failure Symptom Checkl } \\
(\text { mean } \pm S D)\end{array}$ & $t(0.00=$ not bothere & at all, I.00 = ver & ered) \\
\hline & Cardiopulmonary & $0.37(0.18)$ & $0.23(0.18)$ & 0.002 \\
\hline & Gastrointestinal & $0.26(0.12)$ & $0.19(0.14)$ & 0.004 \\
\hline & Psychological & $0.25(0.20)$ & $0.20(0.19)$ & ns \\
\hline & Genitourinary & $0.23(0.15)$ & $0.14(0.13)$ & 0.002 \\
\hline & Neurological & $0.22(0.12)$ & $0.17(0.11)$ & $0.017(\mathrm{~ns})$ \\
\hline & Dermatological & $0.08(0.08)$ & $0.06(0.08)$ & ns \\
\hline & Physical (all except psychological) & $0.22(0.09)$ & $0.16(0.09)$ & 0.001 \\
\hline & Total score & $0.23(0.10)$ & $0.16(0.10)$ & 0.002 \\
\hline & $\begin{array}{l}\text { Functional disability from the } \mathbf{S} \\
\text { most disability) (mean } \pm S D)\end{array}$ & kness Impact Profil & $0.00=$ least disa & $1.00=$ \\
\hline & Recreation & $0.54(0.30)$ & $0.46(0.30)$ & ns \\
\hline & Home management & $0.49(0.32)$ & $0.43(0.39)$ & ns \\
\hline & Work & $0.48(0.09)$ & $0.50(0.00)$ & ns \\
\hline & Sleep/rest & $0.40(0.29)$ & $0.43(0.29)$ & ns \\
\hline & Social interaction & $0.35(0.20)$ & $0.34(0.25)$ & ns \\
\hline & Mobility & $0.34(0.24)$ & $0.29(0.27)$ & ns \\
\hline & Ambulation & $0.30(0.17)$ & $0.35(0.22)$ & ns \\
\hline & Alertness & $0.23(0.33)$ & $0.20(0.24)$ & ns \\
\hline & Self-care & $0.20(0.15)$ & $0.30(0.15)$ & 0.002 \\
\hline & Eating & $0.17(0.11)$ & $0.14(0.11)$ & ns \\
\hline & Emotional behaviour & $0.12(0.15)$ & $0.17(0.22)$ & ns \\
\hline & Communication & $0.10(0.18)$ & $0.17(0.25)$ & ns \\
\hline & Psychological disability & $0.24(0.16)$ & $0.25(0.19)$ & ns \\
\hline & Physical disability & $0.34(0.13)$ & $0.35(0.14)$ & ns \\
\hline & Total score & $0.30(0.13)$ & $0.32(0.14)$ & ns \\
\hline \multicolumn{5}{|c|}{ Second-generation LVADs } \\
\hline \multicolumn{5}{|c|}{ No studies of second-generation devices } \\
\hline
\end{tabular}


$(0.00=$ not bothered at all, $1.00=$ very bothered $)$, the mean total score improved significantly $(p=0.002)$ from $0.23(0.10)$ before to $0.16(0.10)$ after implantation of the LVAD. The subcategories of cardiopulmonary (before 0.37 , after 0.23; $p=0.002$ ), gastrointestinal (before 0.26, after $0.19 ; p=0.004$ ), genitourinary (before 0.23 , after $0.14 ; p=0.002$ ), neurological (before 0.22 , after $0.17 ; p=0.017$ ) and physical (before 0.22 , after $0.16 ; p=0.001$ ) improved significantly from pre- to post-implantation of the LVAD. Improvements in the subcategories of psychological (before 0.25 , after $0.20 ; p=\mathrm{ns}$ ) and dermatological (before 0.08 , after $0.06 ; p=\mathrm{ns}$ ) improved but not significantly. Patients were generally 'less bothered' than 'more bothered' by symptoms both before and after device implant as scores were all below the mean of 0.50 .

The mean (SD) total score on functional disability from the Sickness Impact Profile $(0.00=$ least disability to $1.00=$ most disability) worsened in patients following implantation of the LVADs with an insignificant increase $(p=\mathrm{ns})$ in the index from $0.30(0.13)$ before implant and $0.32(0.14)$ after implant. Only the self-care subcategory was found to worsen significantly $(p=0.002)$, increasing from $0.20(0.15)$ before to $0.30(0.15)$ after LVAD implantation. Similarly the subcategories of work (before 0.48 , after 0.50 ), sleep/rest (before 0.40 , after 0.43 ), ambulation (before 0.30 , after 0.35 ), emotional behaviour (before 0.12, after 0.17), communication (before 0.10 , after 0.17 ), psychological disability (before 0.24 , after 0.25 ) and physical disability (before 0.34 , after 0.35 ) worsened, although not significantly $(p=\mathrm{ns})$. In contrast, the subcategories of recreation (before 0.54, after 0.46), home management (before 0.49, after 0.43 ), social interaction (before 0.35 , after 0.34 ), mobility (before 0.34 , after 0.29 ), alertness (before 0.23, after 0.20) and eating (before 0.17, after 0.14$)$ improved but not significantly $(p=\mathrm{ns})$.

On the global ratings of different areas of QoL from the Rating Question Form there were significant improvements in the mean (SD) QoL $(1=$ very poor, $10=$ very good) [before $3.5(2.5)$, after $5.9(2.7) ; p=0.002]$ and health $(1=$ very poor, $10=$ very good) [before 4.0 (3.1), after 6.2 (2.4); $p=0.012]$. In contrast, patients' response to 'How well will do/doing after LVAD' ( 1 = very poorly, $10=$ very well) worsened significantly [before $8.7(1.3)$, after $7.1(2.1) ; p=0.001$ ], as it did for coping ability $(1=$ very poorly, $10=$ very well) [before $7.7(2.0)$, after $6.6(2.6) ; p=0.026$ ] Stress levels $(1=$ no stress, $10=$ very much stress $)$ improved [before 6.2 (2.5), after $4.9(2.9) ; p=\mathrm{ns}$ ] but not significantly. Patients' response to 'How well will do/doing after heart transplant' $(1=$ very poorly, $10=$ very well) did not change [before 9.3 (0.9), after $9.3(1.1) ; p=\mathrm{ns}]$.

\section{Second-generation LVADs}

None of the studies of the second-generation devices reported changes in the QoL of patients on recognised outcome measures.

\section{Adverse events}

\section{First-generation LVADs}

HeartMate. Adverse events were reported by the CCT comparing the HeartMate VE LVAD with the Novacor N100 LVAD, ${ }^{80}$ and also the three cohort analytic studies $^{81-83}$ and the three cohort studies ${ }^{84-86}$ assessing the HeartMate LVAD compared with inotropic agents or usual medical care (Table 20 and Appendix 12), focusing on survival of patients without major complications, mortality from adverse events, rates of infections and mechanical failures associated with the different devices.

Mortality from adverse events El Banayosy and colleagues $^{80}$ reported deaths from adverse events of patients while supported by either the HeartMate VE LVAD $(n=20)$ or the Novacor N100 LVAD $(n=20)$. Six patients with HeartMate LVADs died [multiorgan failure (MOF)/sepsis, five patients; cerebral bleeding, one patient] compared with four patients supported by the Novacor N100 LVAD (MOF/sepsis, three patients; cerebral bleeding, one patient) ( $p$ not stated).

Patients receiving the HeartMate LVAD were less likely to suffer or die from major complications either before or after heart transplantation than those patients on inotropic agents or usual medical care. Bank and colleagues ${ }^{83}$ reported a significantly $(p<0.05)$ higher proportion of patients with LVADs $(55.6 \%, n=18)$ surviving to 6 months without major complications posttransplant than for patients receiving inotropic agents $(15.8 \%, n=19)$. In comparing the use of LVADs with inotropic agents, Aaronson and colleagues $^{82}$ found a higher pre- (LVADs $18 \%$, inotropes $26 \%$; $p$ not stated) and post- (LVADs $4 \%$, inotropes $32 \% ; p=0.045$ ) heart transplant mortality among those patients receiving inotropic agents. The underlying causes of pre-heart transplant mortality were cerebrovascular accident (LVADs 1, inotropes 1), device failure (LVADs 1), haemorrhage (LVADs 1), MOF and/or sepsis (LVADs 5, inotropes 4), right-sided circulatory failure (LVADs 4), sudden death (inotropes 2) and refractory cardiogenic shock (inotropes 3), whereas the cause of post-transplant mortality was 
TABLE 20 Summary of evidence of adverse events following treatment with first-generation LVADs of patients with ESHF as a BTT

\begin{tabular}{|c|c|c|c|c|}
\hline Study & Outcome & Intervention & Comparison & p-Value \\
\hline \multirow[t]{16}{*}{$\begin{array}{l}\text { HeartMate LVADs } \\
\text { El Banayosy et al., } 2000^{80}\end{array}$} & & Novacor $100 \mathrm{~N}$ & HeartMate LVAD & \\
\hline & Death (\%) & $\begin{array}{l}\text { 4/20 (20) } \\
\text { MOF/sepsis: } 3 \\
\text { Thromboembolism: I }\end{array}$ & $\begin{array}{l}\text { 6/20 (30) } \\
\text { MOF/sepsis: } 5 \\
\text { Cerebral bleeding: I }\end{array}$ & Not stated \\
\hline & \multicolumn{4}{|c|}{ Neurological complications and device-related infections: (events/patient/month, $95 \% \mathrm{Cl}$ ): } \\
\hline & Thromboembolic event (\%) & $\begin{array}{l}4(20)(0.026,0.00 I \\
\text { to } 0.05 \mathrm{I}) \\
\text { (on days } 14-67, \\
\text { mean } 29 \text { days) }\end{array}$ & 0 & 0.1 \\
\hline & Driveline infection (\%) & $\begin{array}{l}4(20)(0.026,0.0 \mathrm{I} \\
\text { to } 0.05 \mathrm{I})\end{array}$ & $\begin{array}{l}9(45)(0.078,0.029 \\
\text { to } 0.127)\end{array}$ & 0.09 \\
\hline & Pocket infection (\%) & $\begin{array}{l}2(10)(0.013,-0.005 \\
\text { to } 0.031)\end{array}$ & $\begin{array}{l}5(25)(0.044,0.006 \\
\text { to } 0.082)\end{array}$ & 0.1 \\
\hline & $\begin{array}{l}\text { Device-related infections } \\
\text { (days 30-111, mean } 58 \text { days) (\%) }\end{array}$ & $\begin{array}{l}4(20)(0.025 \\
0.001 \text { to } 0.05 I)\end{array}$ & $\begin{array}{l}11(55)(0.096 \\
0.042 \text { to } 0.15)\end{array}$ & 0.02 \\
\hline & Controller exchange (technical) (\%) & $\begin{array}{l}2(10)(0.013 \\
-0.005 \text { to } 0.031)\end{array}$ & $\begin{array}{l}14(70)(0.122 \\
0.062 \text { to } 0.182)\end{array}$ & $<0.001$ \\
\hline & Driveline crack (\%) & $\begin{array}{l}3(15)(0.019 \\
-0.003 \text { to } 0.041)\end{array}$ & $\begin{array}{l}2(10)(0.017 \\
-0.007 \text { to } 0.04 I)\end{array}$ & 0.6 \\
\hline & Pump failure (\%) & 0 & $\begin{array}{l}4(20)(0.035 \\
0.001 \text { to } 0.069)\end{array}$ & 0.3 \\
\hline & Other complications & & & \\
\hline & Bleeding & $8(40 \%)$ & $7(35 \%)$ & 0.7 \\
\hline & Reoperation for bleeding & $6(30 \%)$ & $4(20 \%)$ & 0.5 \\
\hline & Systemic infection & $4(20 \%)$ & $9(45 \%)$ & \\
\hline & $\begin{array}{l}\text { Right heart failure necessitating } \\
\text { RVAD support }\end{array}$ & I (5\%) & I (5\%) & \\
\hline & $\begin{array}{l}\text { Right heart failure with medical } \\
\text { treatment }\end{array}$ & $4(20 \%)$ & $2(10 \%)$ & 0.4 \\
\hline \multirow[t]{12}{*}{ Massad et al., $1996^{81}$} & Post-heart transplant & HeartMate LVAD & Medical control & \\
\hline & 30-day operative mortality (\%) & $2(3.8)$ & $9(4.4)$ & ns \\
\hline & Re-exploration for bleeding (\%) & $3(5.7)$ & $9(4.4)$ & ns \\
\hline & Septicaemia (\%) & $\begin{array}{l}\text { Successful BTT: } \\
27 / 53(5 \mathrm{I})\end{array}$ & Not stated & Not stated \\
\hline & $\begin{array}{l}\text { Septicaemia from device-related } \\
\text { infection (\%) }\end{array}$ & $\begin{array}{l}\text { Successful BTT: } \\
21 / 53(40)\end{array}$ & Not stated & Not stated \\
\hline & $\begin{array}{l}\text { Abdominal complications } \\
\text { necessitating operative intervention }\end{array}$ & $\sim 15 \%$ & Not stated & Not stated \\
\hline & Adverse events at I year & & & \\
\hline & CMV infection & $20 \%(n=53)$ & $17 \%(n=203)$ & $\begin{array}{l}\text { Kaplan- } \\
\text { Meier ns }\end{array}$ \\
\hline & Vascular rejection rate & $15 \%(n=53)$ & $12 \%(n=203)$ & $\begin{array}{l}\text { Kaplan- } \\
\text { meier ns }\end{array}$ \\
\hline & Moderate and severe rejection free & $12 \%(n=53)$ & $22 \%(n=203)$ & ns \\
\hline & Transplant CAD free & $90 \%(n=53)$ & $88 \%(n=203)$ & ns \\
\hline & $\begin{array}{l}\text { Mean no. of moderate and severe } \\
\text { rejection episodes }\end{array}$ & $\begin{array}{l}\text { At } 12 \text { weeks: } 1.68 \\
\text { At I year: } 2.53\end{array}$ & $\begin{array}{l}\text { At I } 2 \text { weeks: } 1.47 \\
\text { At I year: } 1.99\end{array}$ & ns \\
\hline
\end{tabular}


TABLE 20 Summary of evidence of adverse events following treatment with first-generation LVADs of patients with ESHF as a BTT (cont'd)

\begin{tabular}{|c|c|c|c|c|}
\hline Study & Outcome & Intervention & Comparison & p-Value \\
\hline \multirow[t]{3}{*}{ Aaronson et al., $2002^{82}$} & & HeartMate LVAD & Inotropic agent & \\
\hline & $\begin{array}{l}\text { Pre-transplant mortality, cause } \\
\text { of death }(\%)\end{array}$ & $\begin{array}{l}\text { I2/66 (I8) } \\
\text { Cerebrovascular } \\
\text { accident I } \\
\text { Device failure I } \\
\text { Haemorrhage I } \\
\text { MOF/sepsis } 5 \\
\text { Right-sided } \\
\text { circulatory failure } 4 \\
\text { All occurred by } \\
19 \text { days after LVAD } \\
\text { implant }\end{array}$ & $\begin{array}{l}\text { I0/38 (26) } \\
\text { Cerebrovascular } \\
\text { accident I } \\
\text { MOF/sepsis } 4 \\
\text { Sudden death } 2 \\
\text { Refractory } \\
\text { cardiogenic } \\
\text { shock } 3\end{array}$ & Not stated \\
\hline & $\begin{array}{l}\text { Post-transplant mortality, cause } \\
\text { of death }(\%)\end{array}$ & $\begin{array}{l}\text { 2/48 (4) } \\
\text { Cerebrovascular } \\
\text { accident I } \\
\text { Rejection (acute) I }\end{array}$ & $\begin{array}{l}\text { 9/28 (32) } \\
\text { Cerebrovascular } \\
\text { accident I } \\
\text { Infection } 3 \\
\text { Haemorrhage I } \\
\text { Primary allograft } \\
\text { dysfunction I } \\
\text { Rejection (acute) } 3\end{array}$ & 0.045 \\
\hline \multirow[t]{19}{*}{ Bank et al., $2000^{83}$} & & HeartMate LVAD & Inotropic agents & \\
\hline & Death before transplant & $\begin{array}{l}\text { I/20 (sepsis) } \\
\text { I/20 }\end{array}$ & $1 / 20$ & Not stated \\
\hline & None & $8 / 20(40 \%)$ & II/20 (55\%) & Not stated \\
\hline & Acute renal failure & 0 & 0 & Not stated \\
\hline & Right heart failure & 0 & 0 & Not stated \\
\hline & Reoperation & $\mathrm{I} / 20(5)$ & $0 / 20$ & Not stated \\
\hline & Mechanical device failure & $4 / 20(20)$ & $\mathrm{N} / \mathrm{A}$ & Not stated \\
\hline & Infection & $9 / 20(45)$ & $8 / 20(40)$ & Not stated \\
\hline & Stroke & $1 / 20(5)$ & $0 / 20$ & Not stated \\
\hline & Adverse effects post-transplant (\%) ( $\mathrm{r}$ & $=18 L V A D, \mathrm{n}=19 \mathrm{cc}$ & trol) & \\
\hline & None & II (6I.I) & $3(15.8)$ & Not stated \\
\hline & Acute renal failure & $3(16.7)$ & $10(52.6)$ & $<0.05$ \\
\hline & Right heart failure & $\mathrm{I}(5.6)$ & $6(31.6)$ & $<0.05$ \\
\hline & Reoperation & $3(16.7)$ & $7(36.8)$ & ns \\
\hline & Rejection & I (5.6) & $3(15.7)$ & ns \\
\hline & Disability & $2(I I . I)$ & $4(2 \mid .0)$ & ns \\
\hline & Infection & $3(16.7)$ & $8(42.1)$ & ns \\
\hline & Stroke & I (5.6) & I (5.2) & ns \\
\hline & Death & $2(11.1)$ & $5(26.3)$ & ns \\
\hline \multirow[t]{6}{*}{ Frazier et al., $1992^{85}$} & & HeartMate LVAD & Medical care & \\
\hline & $\begin{array}{l}\text { Bleeding requiring return to } \\
\text { operating room }\end{array}$ & 0 & $\mathrm{~N} / \mathrm{A}$ & $\mathrm{N} / \mathrm{A}$ \\
\hline & $\begin{array}{l}\text { Patient-related bleeding, e.g. } \\
\text { cardiac tamponade }(\%)\end{array}$ & II (39) & $\mathrm{N} / \mathrm{A}$ & $\mathrm{N} / \mathrm{A}$ \\
\hline & $\begin{array}{l}\text { Haemolysis (before and after LVAD } \\
\text { implantation) (\%) }\end{array}$ & I (4) & $\mathrm{N} / \mathrm{A}$ & N/A \\
\hline & Haematocrit & $\begin{array}{l}\text { After implant: mean } \\
34 \%\end{array}$ & N/A & N/A \\
\hline & & & & continued \\
\hline
\end{tabular}


TABLE 20 Summary of evidence of adverse events following treatment with first-generation LVADs of patients with ESHF as a BTT (cont'd)

\begin{tabular}{|c|c|c|c|c|}
\hline Study & Outcome & Intervention & Comparison & $p$-Value \\
\hline & Platelet count & $\begin{array}{l}\text { After implant: mean } \\
249,000 / \mathrm{ml}\end{array}$ & $\mathrm{N} / \mathrm{A}$ & $\mathrm{N} / \mathrm{A}$ \\
\hline & Free plasma haemoglobin $(n=26)$ & $\begin{array}{l}\text { After implant: mean } \\
8.7 \mathrm{mg} / \mathrm{dl}\end{array}$ & $\mathrm{N} / \mathrm{A}$ & $\mathrm{N} / \mathrm{A}$ \\
\hline & & $\begin{array}{l}\text { Haemoglobin conc. } \\
\text { II g/dl }\end{array}$ & & \\
\hline & Infection (\%) & $\begin{array}{l}7(25) \\
\text { (6 underwent } \\
\text { transplantation, } \\
4 \text { long-term } \\
\text { survivors). } \\
\text { Infected vs } \\
\text { non-infected: } \\
P>0.05 \text { for } \\
\text { outcome }\end{array}$ & $2(33)$ & Not stated \\
\hline & $\begin{array}{l}\text { Renal or hepatic dysfunction or } \\
\text { both before or during LVAD } \\
\text { (not considered device-related) } \\
\text { (\%) }\end{array}$ & $\begin{array}{l}\text { 24/26 LVAD group } \\
(92.3)\end{array}$ & $\mathrm{N} / \mathrm{A}$ & $\mathrm{N} / \mathrm{A}$ \\
\hline & $\begin{array}{l}\text { Right heart failure (required right } \\
\text { ventricular assistance or exhibited } \\
\text { symptoms of serious right } \\
\text { ventricular dysfunction after LVAD } \\
\text { implantation) (\%) }\end{array}$ & $6(2 I)$ & $\mathrm{N} / \mathrm{A}$ & $\mathrm{N} / \mathrm{A}$ \\
\hline & $\begin{array}{l}\text { Thromboembolism device related } \\
\text { Related to mechanical aortic valve } \\
\text { in natural heart (\%) }\end{array}$ & $\begin{array}{l}0 \\
\text { I (4) (successful } \\
\text { transplantation) }\end{array}$ & $\mathrm{N} / \mathrm{A}$ & $\mathrm{N} / \mathrm{A}$ \\
\hline & Bowel adhesions to drive line (\%) & $2(7)$ & $\mathrm{N} / \mathrm{A}$ & $\mathrm{N} / \mathrm{A}$ \\
\hline & $\begin{array}{l}\text { Mechanical failure (loose outflow } \\
\text { connector) (\%) }\end{array}$ & I (4) & N/A & $\mathrm{N} / \mathrm{A}$ \\
\hline \multirow[t]{5}{*}{ Frazier et al., $1994^{86}$} & & HeartMate LVAD & $\begin{array}{l}\text { Medical care } \\
\text { (unless stated } \\
\text { otherwise) }\end{array}$ & \\
\hline & Mortality (\%) & $\begin{array}{l}2(10) \text { (during } \\
\text { extended support) }\end{array}$ & $3(25)$ & Not stated \\
\hline & $\begin{array}{l}\text { Possible device related axillary } \\
\text { artery thromboembolus plus } \\
\text { transient ischaemic attack } \\
\text { (no long-term sequelae) (\%) }\end{array}$ & I (5) & $\mathrm{N} / \mathrm{A}$ & Not stated \\
\hline & $\begin{array}{l}\text { Episodes of rejection per patient } \\
\text { up to } 6 \text {-months post-transplant }\end{array}$ & $\begin{array}{l}0.71 \text { episodes } \\
\text { (SD 0.98) }\end{array}$ & $\begin{array}{l}\text { No data for } \\
\text { control group } \\
\text { General transplant } \\
\text { population: } \\
\text { I.19 episodes } \\
\text { (SD I.0) }\end{array}$ & Not stated \\
\hline & Severe rejection & $\begin{array}{l}0.13 \text { episodes } \\
(S D 0.087)\end{array}$ & $\begin{array}{l}\text { No data for control } \\
\text { group } \\
\text { General transplant } \\
\text { population: } \\
0.35 \text { episodes } \\
\text { (SD 0.02) }\end{array}$ & Not stated \\
\hline
\end{tabular}


TABLE 20 Summary of evidence of adverse events following treatment with first-generation LVADs of patients with ESHF as a BTT (cont'd)

\begin{tabular}{|c|c|c|c|c|}
\hline Study & Outcome & Intervention & Comparison & p-Value \\
\hline & \multicolumn{4}{|c|}{$\begin{array}{l}\text { Patients with } \geq I \text { infectious episodes during } 6 \text { months after transplant (no information is } \\
\text { provided about the number of patients on which percentages are based) }(\%)\end{array}$} \\
\hline & Bacterial & 40 & 36 & Not stated \\
\hline & Viral & 7 & 44 & \\
\hline & Fungal & 7 & 20 & \\
\hline & Protozoal & 0 & 7 & \\
\hline \multirow[t]{5}{*}{ Grady et al., $2001^{84}$} & & $\begin{array}{l}\text { HeartMate LVAD } \\
\text { before implantation }\end{array}$ & $\begin{array}{l}\text { I-2 weeks after } \\
\text { implantation }\end{array}$ & \\
\hline & \multicolumn{4}{|c|}{ Post-implant complications 30 days postoperation } \\
\hline & Mechanical device & $23 \%$ & Not stated & Not stated \\
\hline & Infection & $83 \%$ & Not stated & Not stated \\
\hline & Psychiatric complications & $40 \%$ & Not stated & Not stated \\
\hline \multicolumn{5}{|l|}{ Novacor LVADs } \\
\hline \multirow[t]{8}{*}{ Trachiotis et al., $2000^{87}$} & & $\begin{array}{l}\text { Novacor LVAD } \\
<30 \text { days }\end{array}$ & $\begin{array}{l}\text { Novacor LVAD } \\
>30 \text { days }\end{array}$ & \\
\hline & Infection & $5(100)$ & $4(80)$ & Not stated \\
\hline & Pancreatitis & I (20) & 0 & Not stated \\
\hline & Reoperation (bleeding) & $3(60)$ & 0 & Not stated \\
\hline & Thromboembolism & I (20) & $4(80)$ & Not stated \\
\hline & Neurological dysfunction & $2(40)$ & $3(60)$ & Not stated \\
\hline & Renal failure/dialysis & $\mathrm{I} / 0(20 / 0)$ & $2 / 1(40 / 20)$ & Not stated \\
\hline & Right heart failure & 0 & 0 & Not stated \\
\hline \multicolumn{5}{|l|}{ Toyobo LVADs } \\
\hline Masai et al., $1995^{88}$ & \multicolumn{4}{|c|}{ Adverse events were not reported separately for LVAD patients and BiVAD patients } \\
\hline \multirow{4}{*}{$\begin{array}{l}\text { Thoratec LVADs } \\
\text { Holman et al., } 1995^{89}\end{array}$} & & & & \\
\hline & Adverse events from Thorat & & No comparator & \\
\hline & $\begin{array}{l}\text { On second postoperative } \\
\text { VAD output during this wa } \\
\text { systolic blood pressure wa } \\
\text { and intravenous amiodaro }\end{array}$ & $\begin{array}{l}\text { isode of VT. } \\
\text { itres/minute and } \\
\mathrm{mHg} \text {. Cardioverted } \\
\text { tinued. }\end{array}$ & & $\mathrm{N} / \mathrm{A}$ \\
\hline & $\begin{array}{l}\text { Episodes of paroxysmal } \mathrm{V} \\
\text { and became more frequen }\end{array}$ & $\begin{array}{l}\text { sional VF recurred } \\
\text { le ensuing days }\end{array}$ & & \\
\hline \multirow[t]{2}{*}{ May and Adams, $1987^{90}$} & Adverse events from Thorat & & No comparator & \\
\hline & \multicolumn{3}{|c|}{$\begin{array}{l}\text { Severe respiratory distress after } 2.5 \text { days post-LVAD } \\
\text { implant, and developed adult respiratory distress syndrome. } \\
\text { Intubated and placed on } 100 \% \mathrm{O}_{2} \text { and } 12-15 \mathrm{~cm} \text { of } \\
\text { positive end-expiratory pressure but was only able to } \\
\text { maintain an arterial partial pressure of oxygen of } 50 \mathrm{mmHg} \text {. } \\
\text { Blood cultures showed Legionella bacteria, treated with } \\
\text { antibiotics. Haemolysis which stabilised in I week }\end{array}$} & $\mathrm{N} / \mathrm{A}$ \\
\hline
\end{tabular}


cerebrovascular accident (LVADs 1, inotropes 1), acute rejection (LVADs 1 , inotropes 3 ), infection (inotropes 3), haemorrhage (inotropes 1) and primary allograft dysfunction (inotropes 1). Bank and colleagues ${ }^{83}$ reported two deaths (10\%) among patients receiving LVADs (one patient died of sepsis and one of ventricular fibrillation following device removal) and one death (5\%) among those on inotropes [refractory ventricular tachycardia (VT)] prior to heart transplantation. After heart transplantation, Bank and colleagues ${ }^{83}$ noted that two patients $(10 \%)$ with LVADs died compared with five patients $(25 \%)$ receiving inotropes; the causes of death are not discussed. In the comparison of LVADs and usual medical care, Massad and colleagues ${ }^{81}$ reported similar 30-day operative mortality following heart transplantation with $3.8 \%(n=53)$ of patients with LVADs and $4.4 \%(n=203)$ of patients receiving usual medical care dying $(p=\mathrm{ns})$.

Frazier and colleagues ${ }^{86}$ cohort study noted lower mortality rates prior to heart transplantation among the 19 patients with LVADs (10\%) than for the 12 patients receiving usual medical care $(25 \%)$. Mortality among the LVAD patients was from MOF associated with systemic lupus from the pre-implantation period (one patient) and from a massive thromboembolic embolism (one patient). None of the patients with an LVAD died post-heart transplantation, whereas the three patients with usual medical care who received a heart transplant died (two patients immediately postoperation from massive bleeding and donor heart failure and one patient from lymphoma-related rejection).

Infection Five studies reported the occurrence of infections following implantation of the HeartMate LVAD. ${ }^{80,81,83-85}$ El Banayosy and colleagues ${ }^{80}$ reported higher rates of infection for patients supported by the HeartMate VE LVAD $(n=20)$ compared with the Novacor N100 LVAD $(n=20)$. The occurrence of driveline infections was significantly $(p=0.09)$ higher among patients receiving a HeartMate VE LVAD [nine patients; 0.078 events/patient/month $(95 \%$ CI 0.029 to 0.127)] than with the Novacor N100 LVAD [four patients; 0.026 events/patient/month (95\% CI 0.01 to 0.051)]. Similarly, El Banayosy and colleagues reported a higher occurrence of pocket infections (HeartMate five patients, Novacor two patients) and systemic infections (HeartMate nine patients, Novacor four patients) among patients with a HeartMate VE LVAD than with a Novacor N100 LVAD, although differences were not significant $(p=0.1)$. Infection was the cause of readmission to hospital. Six patients (five pocket infections; one driveline infection) with the HeartMate VE LVAD and four patients (two pocket infections; two driveline infections) with the Novacor N100 LVAD were readmitted to hospital owing to infection (differences were not statistically significant). Bank and colleagues ${ }^{83}$ found similar rates of major infection among patients with LVADs $(45 \%, n=20)$ and with inotropic agents $(40 \%, n=20)$ prior to heart transplantation ( $p$ not stated). Among the group of LVAD patients, the major infections were driveline infections (five patients), pneumonia (three patients) and bacterial sepsis (one patient), wheareas those among the inotrope group of patients were line sepsis (seven patients) and pneumonia (one patient). The occurrence of major infections following heart transplantation were higher among the patients on inotropes $(42 \%$, $n=19)$ compared with the LVAD patients $(16 \%$, $n=18)(p=n s)$. No details are provided of the causes of infections following heart transplantation. Similarly, Frazier and colleagues ${ }^{85}$ found higher rates of infection among patients receiving usual medical care $(33 \%, n=6)$ compared with those with an LVAD $(25 \%, n=28)$. Frazier and colleagues $^{85}$ note that all the infections suffered by the LVAD patients were device related. This study used a historical control group. In their comparison of LVADs with usual medical care, Massad and colleagues $^{81}$ reported the occurrence of septicaemia in patients with an LVAD. Of the 53 patients successfully bridged to heart transplantation, $51 \%$ had septicaemia with $40 \%$ from device-related infection. Grady and colleagues ${ }^{84}$ noted that $83 \%$ of patients with an LVAD who completed an assessment before and after device implantation $(n=30)$ suffered infection during the 30 days following the operation.

Mechanical failure Mechanical device failures were reported in four of the studies of the HeartMate LVAD. ${ }^{80,83-85}$ El Banayosy and colleagues $^{80}$ reported several device-related failures in their comparison of the HeartMate VE LVAD and the Novacor N100 LVAD. Significantly $(p<0.001)$ more patients required the controllers for their HeartMate VE LVAD (14 patients) exchanging than did patients with Novacor LVAD (two patients). Driveline cracks were found in the devices of two HeartMate LVAD and three Novacor LVAD patients $(p=0.6)$. Whereas there were no pump failures among patients with Novacor N100 LVADs, four patients with HeartMate LVADs suffered pump failure $(p=0.3) .{ }^{80}$ As a consequence of the device-related failures, three patients with a HeartMate LVAD and one Novacor patient were readmitted to hospital $(p=0.3)$. Bank and colleagues ${ }^{83}$ noted a 
mechanical device failure rate of $20 \%$ among the 20 patients receiving a HeartMate LVAD, including one patient each suffering a broken console, loss of sensor function, torn inflow housing structures and inflow valve dysfunction due to pannus growth. Grady and colleagues ${ }^{84}$ reported that $23 \%$ of patients receiving a HeartMate LVAD incurred device failure $(n=30)$; no details were provided on the cause. Frazier and colleagues $^{85}$ reported one patient with a loose connector flow from the 34 patients with an LVAD.

\section{Bleeding, reoperation and thromboembolic} events El Banayosy and colleagues ${ }^{80}$ reported that $35 \%$ of patients with a HeartMate LVAD $(n=20)$ and $40 \%$ of patients with a Novacor LVAD $(n=20)$ suffered from bleeding $(p=0.7)$. Some $20 \%$ of HeartMate patients $(n=20)$ and $30 \%$ of Novacor patients $(n=20)$ required reoperation as a consequence of bleeding $(p=0.5)$. El Banayosy and colleagues ${ }^{80}$ reported that four patients with Novacor LVADs suffered a thromboembolic event compared with none of the HeartMate LVAD patients $(p=0.1)$. Massad and colleagues ${ }^{81}$ found similar proportions of patients $(p=\mathrm{ns})$ requiring re-exploration due to bleeding in the LVAD group $(5.7 \%, n=53)$ and the group receiving usual medical care $(4.4 \%, n=203)$. Following LVAD implantation, Frazier and colleagues ${ }^{85}$ found that $39 \%(n=34)$ of patients suffered bleeding, although none required reoperation. In addition, one patient suffered from haemolysis before and after LVAD implantation and one patient was found to have a thromboembolism related to the mechanical aortic valve in the natural heart and not associated with the LVAD. In another study by Frazier and colleagues, ${ }^{86}$ one patient had a possible device-related axillary artery thromboembolus plus transient ischaemic attack. Bank and colleagues ${ }^{83}$ reported that one patient within an LVAD required reoperation prior to heart transplantation; details were not provided.

\section{Other adverse events El Banayosy and} colleagues $^{80}$ reported that three patients with a HeartMate LVAD $(n=20)$ and five patients with a Novacor LVAD $(n=20)$ suffered right heart failure necessitating support with either a RVAD or medical treatment. Frazier and colleagues ${ }^{85}$ noted that $21 \%(n=34)$ of patients with an LVAD suffered right heart failure requiring right ventricular assistance or exhibited symptoms of serious right ventricular dysfunction after LVAD implantation. Also, Frazier and colleagues ${ }^{85}$ found two patients who suffered from bowel adhesions to the driveline. Bank and colleagues ${ }^{83}$ reported that one patient with an LVAD suffered a stroke prior to heart transplantation, and Grady and colleagues ${ }^{84}$ found that $40 \%$ of patients $(n=30)$ receiving an LVAD suffered from psychiatric complications.

Adverse events after heart transplantation Three studies examined adverse events among the different patient groups following heart transplantation. ${ }^{81,83,86}$ Bank and colleagues reported that $28.9 \%$ of patients with an LVAD $(n=18)$ suffered adverse events after heart transplantation compared with $84.2 \%$ of those receiving inotropic agents $(n=19)$. Significantly more of the patients undergoing treatment with inotropic agents suffered acute renal failure $(52.6 \%$ versus $16.7 \% ; p<0.05)$ or right heart failure $(31.6 \%$ versus $5.6 \% ; p<0.05)$ than patients with an LVAD. Similarly, more patients receiving inotropic agents required reoperation $(36.8 \%$ versus $16.7 \%$ ), suffered rejection $(15.7 \%$ versus $5.6 \%)$, incurred disability (21.0\% versus $11.1 \%$ ) or infections ( $42.1 \%$ versus $16.7 \%)$ than patients with an LVAD, although these differences did not differ significantly. ${ }^{83}$ In addition, one patient in each treatment group suffered a stroke following heart transplantation. Massad and colleagues ${ }^{81}$ reported adverse events 1 year following heart transplantation, with no significant difference $(p=\mathrm{ns})$ between patients with an LVAD $(n=53)$ compared with those on usual medical care $(n=203)$ respectively for cytomegalovirus (CMV) infection (20\% versus $17 \%)$, vascular rejection (15\% versus $12 \%$ ), moderate and severe rejection free $(12 \%$ versus $22 \%)$, transplant coronary artery disease (CAD) free $(90 \%$ versus $88 \%)$ and moderate and severe rejection episodes (2.53 versus 1.99). Frazier and colleagues ${ }^{86}$ compared the adverse events following heart transplantation suffered by patients receiving an LVAD $(n=19)$ with those in the general transplant population $(n=12)$. Up to 6 months after heart transplantation, patients with an LVAD suffered 0.71 episodes of rejection per patient compared with 1.19 episodes in the general transplant population. Similarly, patients with LVADs suffered fewer episodes of severe rejection than the general transplant population with 0.13 compared with 0.35 rejection episodes. ${ }^{86}$ In addition, episodes of infection 6 months after heart transplantation tended to be more evident among those patients receiving usual medical care than an LVAD, whether viral (44\% versus $7 \%$ ), fungal (20\% versus $7 \%$ ) or protozoal (7\% versus $0 \%$ ) infections. Bacterial infections affected $40 \%$ of LVAD patients and $36 \%$ of the general transplant population.

Novacor. In the cohort analytic study comparing patients supported with a Novacor LVAD for 
$<30$ days $(n=5)$ with those supported for more than 30 days $(n=5)$ (Table 20 and Appendix 13 ). Trachiotis and colleagues ${ }^{87}$ reported the occurrence of infection ( 5 versus 4 ), pancreatis (1 versus 0 ), reoperation due to bleeding (3 versus 0 ), thromboembolism (1 versus 4 ), neurological dysfunction (2 versus 3 ), and renal failure/dialysis ( $1 / 0$ versus $2 / 1)$. In addition, Trachiotis and colleagues $^{87}$ reported two late deaths from malignancy and two deaths from sepsis and MOF and one patient had the LVAD removed (reason not stated).

Toyobo. Adverse effects were reported in the case series by Masai and colleagues ${ }^{88}$ of the Toyobo LVAD (Table 20 and Appendix 14). Unfortunately, the adverse events data provided aggregate data for three patients with an LVAD and one patient who was supported with a BiVAD and it is unclear which events relate specifically to the LVAD patients. As a consequence, the adverse events data on cardiovascular accident, postoperative bleeding and mechanical device failure have been excluded from the analysis.

Thoratec. Adverse events were reported in the two case studies of the Thoratec LVAD (Table 20 and Appendix 15). ${ }^{89,90}$ A 45-year-old male patient had an episode of VT 2 days post-implantation. Following that, episodes of paroxysmal VT and occasional ventricular fibrillation (VF) recurred and became more frequent during the ensuing days. ${ }^{89}$ A 24-year-old male patient showed severe respiratory distress after 2.5 days and developed adult respiratory distress syndrome. In addition, this patient had haemolysis which stabilised after 1 week, and was found to have blood cultures positive to Legionella bacteria. $^{90}$

\section{Second-generation LVADs}

Jarvik. The two case series of the Jarvik 2000 LVAD examined adverse events (Table 21 and Appendix 16). ${ }^{94-100}$ Frazier and colleagues ${ }^{94}$ case series of three patients with a Jarvik 2000 LVAD as a BTT found localised infection of the powercable exit site in one patient. There were no device-related medical problems. The second case series ${ }^{95-100}$ of the Jarvik 2000 LVAD reported adverse events for a subset of 10 patients, finding abdominal power cable infections in two patients; a major haemorrhage in one patient, which was from a gastric ulcer and a separate ateriovenous malformation in the small intestine; and technical problems with the LVAD in two patients where the power-cable connectors were broken by the operator in one and one connector pin was bent by the patient. No thromboembolisms in the devices were noted, nor were any device infections or any significant haemolysis.

MicroMed DeBakey. Two cohort studies assessing the MicroMed DeBakey VADs assessed adverse events (Table 21 and Appendix 17). ${ }^{91,93}$ Potapov and colleagues ${ }^{93}$ reported that one of the six patients with a MicroMed DeBakey LVAD died from sepsis. Noon and colleagues ${ }^{91}$ noted that one patient with the LVAD died from a device-related adverse event, although no details are provided.

\section{Summary of clinical effectiveness of LVADs as a bridge to heart transplantation for ESHF Quantity of Studies}

Sixteen studies assessed LVADs as a BTT, with 11 studies of first-generation devices and five studies of second-generation devices. Of the 11 studies of first-generation devices, seven assessed the HeartMate VE/IP LVAD, one the Novacor LVAD, one study the Toyobo LVAD and two the Thoratec LVAD. Direct comparisons were limited to studies of the HeartMate LVAD with alternatives of the Novacor LVAD, ${ }^{80}$ inotropic agents ${ }^{82,83}$ or undefined medical care. ${ }^{81,85,86}$ Of the five studies of second-generation devices, three assessed the MicroMed DeBakey LVAD and two the Jarvik 2000 LVAD. None of these studies included a comparator.

\section{Methodological quality}

The methodological quality of studies was judged to be weak when appraised against criteria that assess the rigor of studies and the opportunities for bias. In many instances this was inevitable given that most studies were observational studies, but the experimental and quasi-experimental studies were also affected by methodological weaknesses that may limit the strength and reliability of the evidence presented.

\section{Generalisability}

Most studies focused on male patients aged between 40 and 60 years who suffered from either dilated or ischaemic cardiomyopathy and were in NYHA Class IV.

\section{Survival}

There was limited difference shown in the CCT of the survival to heart transplantation of patients receiving the HeartMate LVAD (60\% survived, $10 \%$ died) compared with the Novacor LVAD $(65 \%$ survived, $15 \%$ died). ${ }^{80}$ When compared with inotropic agents in two cohort analytic studies, ${ }^{82,83}$ patients receiving the HeartMate LVAD experienced some benefit in actuarial survival to 
TABLE 2 I Summary of evidence of adverse events following treatment with second-generation LVADs of patients with ESHF as a BTT

\begin{tabular}{|c|c|c|c|c|}
\hline Study & Outcome & Intervention & Comparison & $p$-Value \\
\hline \multicolumn{5}{|l|}{ Jarvik 2000 LVADs } \\
\hline \multirow[t]{8}{*}{ Westaby et al., $2002^{95}$} & & Jarvik 2000 LVAD & No comparator & \\
\hline & Abdominal power cable infection & 2 patients & & $\mathrm{N} / \mathrm{A}$ \\
\hline & Major haemorrhage & I patient & & $\mathrm{N} / \mathrm{A}$ \\
\hline & Device thromboembolism & 0 patients & & $\mathrm{N} / \mathrm{A}$ \\
\hline & Device infection & 0 patients & & $\mathrm{N} / \mathrm{A}$ \\
\hline & Significant haemolysis & 0 patients & & $\mathrm{N} / \mathrm{A}$ \\
\hline & $\begin{array}{l}\text { Power-cable connector broken } \\
\text { by patient }\end{array}$ & 2 patients & & $N / A$ \\
\hline & Connector pin bent by patient & I patient & & $\mathrm{N} / \mathrm{A}$ \\
\hline \multirow[t]{9}{*}{ Frazier et al., $2001^{94}$} & & Jarvik 2000 LVAD & No comparator & \\
\hline & Intraoperative blood loss (average) & I.5 litres & & $N / A$ \\
\hline & Postoperative bleeding & $\begin{array}{l}\text { Minimal } \\
\text { (amount not stated) }\end{array}$ & & $\mathrm{N} / \mathrm{A}$ \\
\hline & $\begin{array}{l}\text { Complications associated with } \\
\text { implant surgical procedure }\end{array}$ & $0 / 3$ & & $N / A$ \\
\hline & $\begin{array}{l}\text { Free from adverse events } \\
\text { throughout support }\end{array}$ & I/3 (34\%) & & $\mathrm{N} / \mathrm{A}$ \\
\hline & $\begin{array}{l}\text { Localised infection of power-cable } \\
\text { exit site (responded to antibiotic } \\
\text { therapy and local treatment) }\end{array}$ & I/3 (34\%) & & $N / A$ \\
\hline & $\begin{array}{l}\text { Gastrointestinal bleeding from } \\
\text { duodenal ulcer }\end{array}$ & $1 / 3(34 \%)$ & & $\mathrm{N} / \mathrm{A}$ \\
\hline & Device-related medical problems & $0 / 3$ & & $\mathrm{~N} / \mathrm{A}$ \\
\hline & Thromboembolism & $0 / 3$ & & $\mathrm{~N} / \mathrm{A}$ \\
\hline \multicolumn{5}{|c|}{ MicroMed DeBakey LVADs } \\
\hline \multirow[t]{2}{*}{ Potapov et al., $2000^{93}$} & & $\begin{array}{l}\text { MicroMed } \\
\text { DeBakey LVAD }\end{array}$ & No comparator & \\
\hline & Deaths from sepsis & $1 / 6$ & & $\mathrm{~N} / \mathrm{A}$ \\
\hline \multirow[t]{2}{*}{ Noon et al., 200191 } & & $\begin{array}{l}\text { MicroMed } \\
\text { DeBakey LVAD }\end{array}$ & No comparator & \\
\hline & $\begin{array}{l}\text { Deaths from device-related } \\
\text { adverse events }\end{array}$ & $1 / 32$ & & $\mathrm{~N} / \mathrm{A}$ \\
\hline
\end{tabular}

transplant, which increased with the length of support (actuarial survival: 1 month $78 \%$ versus $81 \%$; 3 months $64 \%$ versus $81 \%$, respectively). The benefit in survival for those receiving a HeartMate LVAD compared with inotropic agents increased post-heart transplantation (survival: 6 months $88.9 \%$ versus $73.3 \%$; actuarial survival: 1 year $98 \%$ versus $74 \%$; 4 years $95 \%$ versus $65 \%$, respectively). Differences in survival when comparing patients who had received the HeartMate LVAD with those on usual care were more equivocal. Whereas a retrospective cohort analytic study found no difference between patients with the HeartMate LVAD and usual care in survival to transplant and retrospective cohort studies with historic controls found an advantage for patients who received an LVAD compared with those on usual care in survival to transplant (64\% versus $50 \%^{85}$ and $84 \%$ versus $25 \%,{ }^{86}$ respectively) and survival posttransplantation (60 days: $52 \%$ versus $17 \%{ }^{85}$; actuarial survival 1 and 2 years: $100 \%$ versus $\left.0 \%{ }^{86}\right)$. The studies of the other devices did not include a comparator intervention and, as a consequence can only provide an indication of the additional survival the patients gained through the specific intervention compared with the assumed outcome of the condition. The retrospective cohort analytic study of the Novacor LVAD compared post-transplantation survival of groups 
supported for $<30$ days with those supported for $\geq 30$ days, with no difference at 3 years $(60 \%$ survival). A case series study of three patients with the Toyobo device showed one patient surviving to 64 days before dying, another patient surviving 119 days to transplantation and a third still alive on the transplant waiting list at 390 days. Two case reports of the Thoratec LVAD showed two patients surviving 21 and 60 days before transplantation. A cohort study of the MicroMed DeBakey LVAD showed that $81 \%$ of patients survived to 30 days post-implantation of the device, $34 \%$ received a heart transplant and $31 \%$ died. In two case series of the Jarvik 2000 LVAD, 70\% had successfully been bridged to transplant with around $30 \%$ having died (one patient was still on support).

\section{Functional status}

Two cohort studies with historical controls of the HeartMate LVAD showed that patients receiving an LVAD were more likely to improve their functional status than those on usual care when assessed on the NYHA functional classification (before LVADs, 100\% NYHA Class IV, usual care 84\% NYHA Class IV; after LVADs, 94\% NYHA Class I, usual care $100 \%$ NYHA Class IV, ${ }^{85}$ and before LVADs, $100 \%$ NYHA Class IV; after LVADs, 94\% NYHA Class I) ${ }^{86}$ Similar improvements in functional status were experienced by patients receiving a second-generation Jarvik 2000 LVAD (before LVAD, 100\% NYHA Class IV; after LVAD, $70 \%$ NYHA Class IV, $30 \%$ dead). ${ }^{95}$

\section{Quality of life}

A cohort study of the HeartMate LVAD provided the only evidence of the effect of first-generation LVADs on QoL. It showed that patients receiving the HeartMate LVAD had statistically significant improvements on some elements of the Quality of Life Index, the Heart Failure Symptom Checklist and the global ratings from the Rating Question Form. However, there was significant worsening of elements of the Quality of Life Index, Rating Question Form and functional disability. No studies of the second-generation LVADs assessed changes in QoL.

\section{Adverse events}

Comparison of the HeartMate and Novacor LVADs showed limited difference in the rates of mortality from adverse events. The HeartMate LVAD appeared to have a higher rate of devicerelated complications, including technical problems with controllers, device-related infections and driveline infections. Other studies of the HeartMate device showed $\sim 20 \%$ of patients having mechanical device failures. ${ }^{83,84}$ Patients with the Novacor LVAD suffered slightly higher rates of bleeding ( $40 \%$ versus 35\%), reoperation (30\% versus $20 \%$ ) and thromboembolic events (20\% versus $0 \%$ ) than those with a HeartMate LVAD. ${ }^{80}$ Other studies have shown lower rates of thromboembolic events for the HeartMate device, but higher rates of bleeding (39\% of patients). ${ }^{85}$ Several studies showed that implantation of the HeartMate device resulted in right heart failure among $\sim 20 \%$ of patients ${ }^{80,85}$ and one study found that $40 \%$ patients suffered psychiatric complications. ${ }^{84}$ Studies of the HeartMate LVAD showed lower rates of adverse events following heart transplantation than for those receiving usual care, with fewer patients suffering end organ failure, reoperation, rejection, disability or infection. Although the adverse events experienced by recipients of other LVADs were less clearly reported, it was evident that patients with the Novacor and Thoratec devices suffered from infection and thromboembolism and required reoperations as a consequence. Studies of the Jarvik 2000 LVAD reported technical problems with power-cable connections breaking and incurring infections, but thromboembolic events, significant device infections or haemolysis were not significant problems. The two cohort studies of the MicroMed DeBakey LVAD reported two device-related deaths from sepsis and an unspecified adverse event.

\section{Conclusion}

LVADs appear to provide some benefit to patients awaiting heart transplantation with an increased chance of surviving both to, and following, the transplantation. Benefits are evident through improvements in the functional status and QoL of patients with an LVAD and appear to outweigh those experienced by patients receiving inotropic agents or usual care. There are risks associated with the use of LVADs with adverse events related to device failures, infections and thromboembolic events. Evidence on the effectiveness of different devices is sparse. Of the first-generation devices only the HeartMate LVAD has evidence comparing it with different alternatives. This shows greater benefits than usual care and inotropic drugs but similar effectiveness when compared with the Novacor LVAD. For the second-generation devices, the early evidence suggests that the Jarvik 2000 and the MicroMed DeBakey LVADs are similar in terms of measures of clinical effectiveness. Although these devices appear to show improvements in the adverse events associated with first-generation devices, experiencing lower rates of infection and thrombolembolism, it is still early in their development to identify whether 
there will be any long-term consequences associated with the change in the nature of the circulatory support.

\section{LVADs as a bridge to recovery}

\section{Quantity of research}

Seven studies considering the clinical effectiveness of LVADs as a BTR met the inclusion criteria for the systematic review (see Table 22 and Appendices 18-22). All the studies assessed firstgeneration devices with one case report of the Abiomed LVAD, ${ }^{104}$ one case report of the HeartMate LVAD, ${ }^{105}$ one case report of the Novacor LVAD ${ }^{106}$, two case reports of the Thoratec LVAD, ${ }^{107,108}$ and two case series evaluations of the Toyobo LVAD. ${ }^{109,110}$ There were no studies of second-generation devices included in the systematic review.

\section{Methodological quality of research First-generation LVADs}

The methodological quality and the quality of reporting of the seven studies were poor (Table 22 and Appendices 18-22). As all the studies were retrospective observational studies with no comparator, there was considerable opportunity for bias. Limited details were provided in the studies concerning patient selection and with the small samples it is unlikely that the patients were representative of the target population. Although the outcomes assessed included survival, mortality and organ function and adverse effects, no details were provided as to the methods of data collection. The quality criteria assessing confounding, blinding and withdrawals/drop-outs were not applicable to these study designs, indicating the opportunity for bias.

\section{Generalisability}

The external validity of the studies was assessed through the key characteristics of the patients assessed (Table 23). As there were very few studies of the different devices, it was unlikely that there would be a representative patient group for any one device. However, it was evident that the patients included were predominantly male (77\%), aged between 16 and 73 years and mainly suffering from dilated cardiomyopathy (40\%) or acute MI and shock (23\%).

\section{Assessment of the clinical effectiveness of LVADs as a bridge to recovery Survival}

\section{First-generation LVADs}

until recovery (Table 24 and Appendix 18). ${ }^{104}$ The 16-year-old patient with cardiogenic shock secondary to acute myocarditis was weaned after 7 days of support and was discharged home. The patient did not require listing for transplantation during the short-term follow-up (duration not known).

HeartMate. The survival of one patient supported with the HeartMate LVAD (no details of type of device) until recovery was reported by Kjellman and colleagues $^{105}$ (Table 24 and Appendix 19). The 19-year-old patient with acute left ventricular heart failure during an exacerbation of multiple sclerosis was supported for 83 days and was then successfully weaned from support. Cardiac function was reported to have normalised. At follow-up at 1 year after explantation, the patient was reported to be in excellent clinical condition without the support of pharmacological therapy for heart failure; cardiac function remained normal and NYHA functional class was I.

Novacor. A case study reported survival of one patient supported with a Novacor LVAD until recovery of heart function (Table 24 and Appendix 20). ${ }^{106}$ The 54-year-old man with cardiac failure refractory to medical therapies and contraindicated to heart transplant was well enough to be transferred to a partial outpatient status 6 weeks after implant and 3 months later was successfully weaned from the device. The patient was discharged home after 6 weeks and was alive and well at 6 months follow-up.

Toyobo. Two case series studies reported survival of patients supported with the Toyobo LVAD (Table 24 and Appendix 22). ${ }^{109,110}$ Nakatani and colleagues $^{109}$ reported survival for five patients supported for up to 11 months. Two of these patients were successfully weaned after 95 days and 50 days of support, respectively. The two patients were still alive at 1 year 6 months and 3 years 9 months, respectively, after explantation of their LVADs. The heart function of the three other patients did not improve and they died at 7 , 9 and 11 months post-LVAD implantation. Noda and colleagues ${ }^{110}$ report survival outcomes for two male patients supported for 12 days. The first patient was weaned at 12 days but then died 149 days later from infection and cerebral haemorrhage. The second patient died from respiratory failure following 12 days of support from the device.

Thoratec. Two case studies reported survival of patients supported with a Thoratec LVAD 


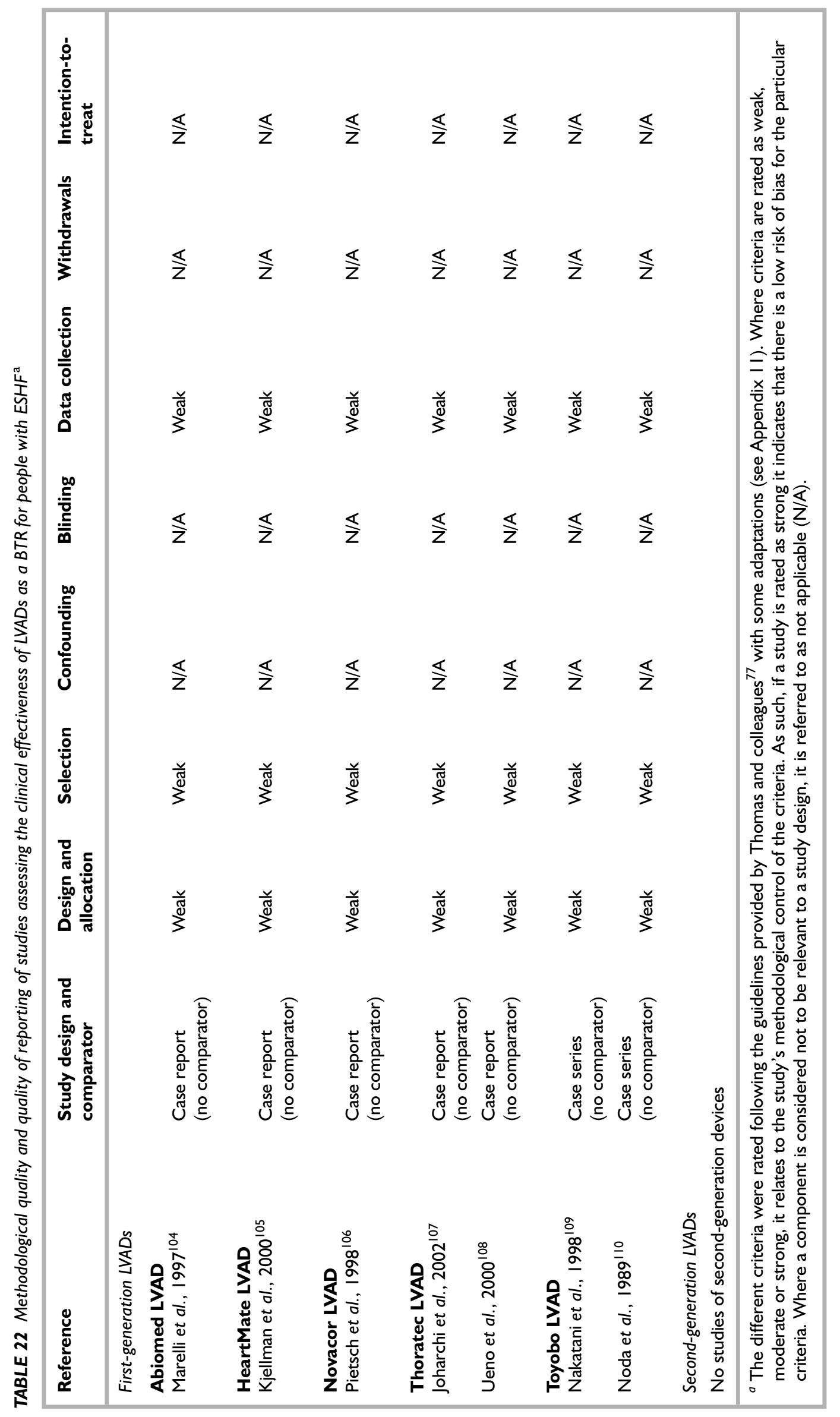




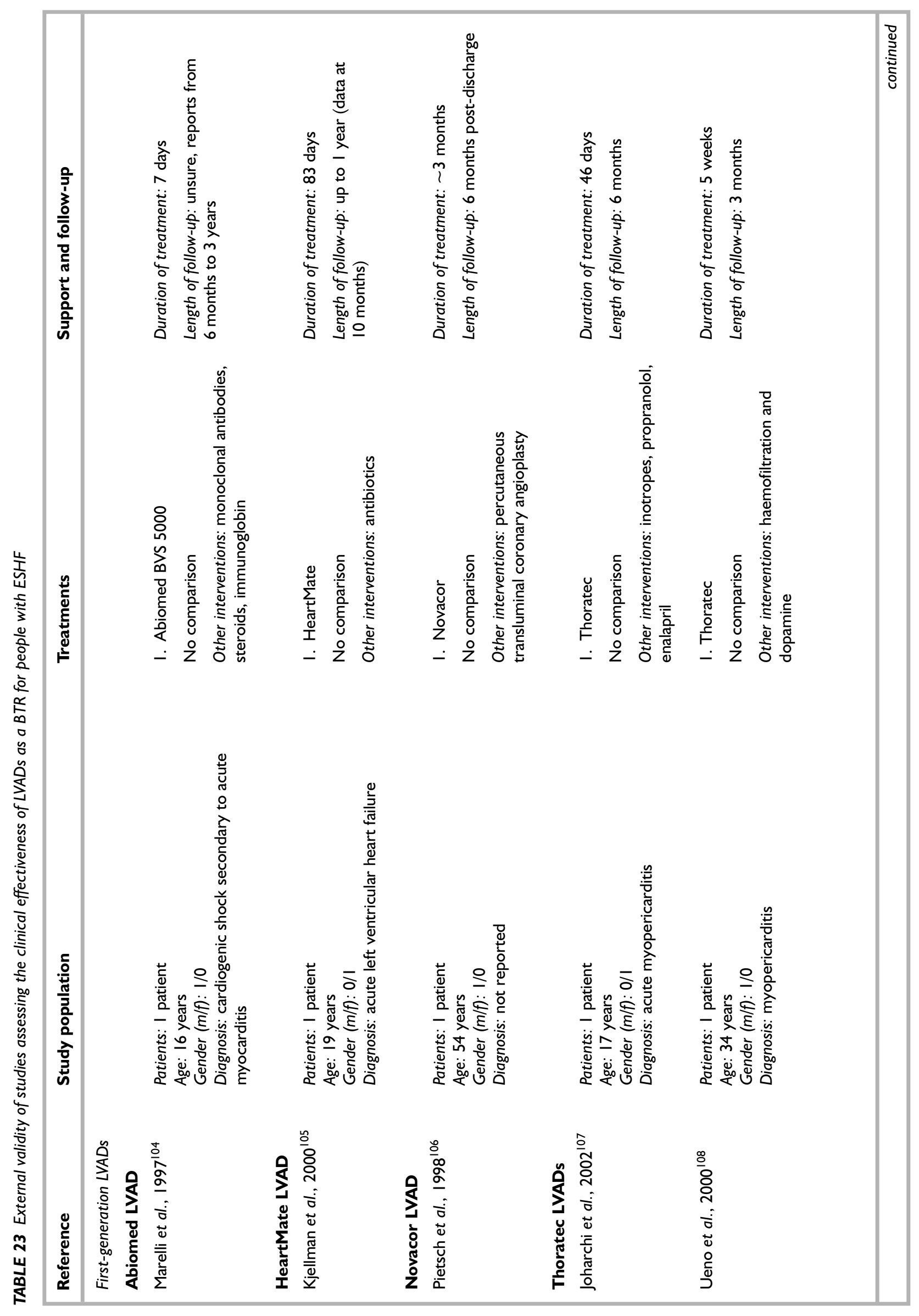




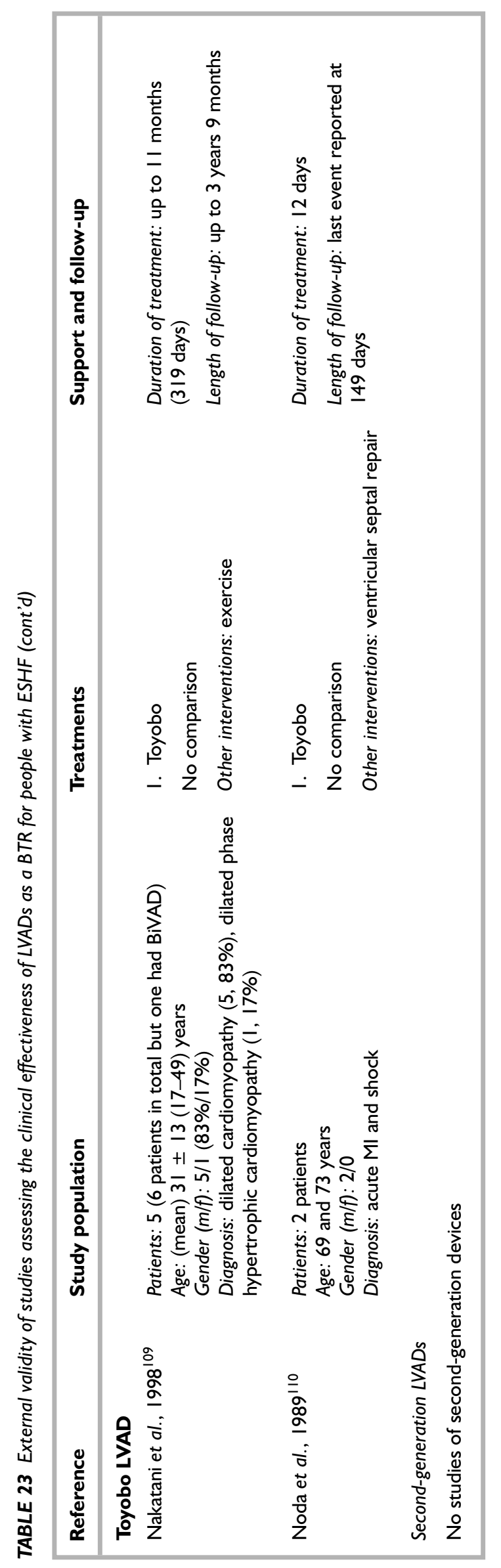


TABLE 24 Summary of evidence of patient survival following treatment of patients with ESHF as a BTR

\begin{tabular}{|c|c|c|}
\hline Study & Outcomes & Intervention \\
\hline \multicolumn{3}{|l|}{ First-generation LVADs } \\
\hline \multicolumn{3}{|l|}{ Abiomed LVADs } \\
\hline Marelli et al., $1997^{104}$ & Survival & Weaned at 7 days and discharged home in good condition \\
\hline \multicolumn{3}{|l|}{ HeartMate LVAD } \\
\hline Kjellman et al., $2000^{105}$ & Survival & $\begin{array}{l}\text { Patient alive I year after explantation, cardiac function normalised and in } \\
\text { NYHA Class I. Not taking any pharmacological of therapy for heart failure }\end{array}$ \\
\hline \multicolumn{3}{|l|}{ Novacor } \\
\hline Pietsch et al., $1998^{106}$ & Survival & $\begin{array}{l}\text { Patient transferred to a partial outpatient status } 6 \text { weeks after implant. } \\
2 \text { months later cardiac recatheterisation. Device removed } 4 \text { weeks after } \\
\text { revascularisation. Discharged home after a further } 6 \text { weeks. Alive at } \\
6 \text { months post-discharge }\end{array}$ \\
\hline \multicolumn{3}{|l|}{ Thoratec LVAD } \\
\hline Joharchi et al., $2002^{107}$ & Survival & $\begin{array}{l}\text { Survived to } 6 \text { months follow-up. Supported for } 46 \text { days when weaned from } \\
\text { LVAD }\end{array}$ \\
\hline Ueno et al., $2000^{108}$ & Survival & Survived to 3 months of follow-up \\
\hline \multicolumn{3}{|l|}{ Toyobo LVAD } \\
\hline Nakatani et al., $1998^{109}$ & Survival & $\begin{array}{l}2 \text { patients survived } 3 \text { months and weaned: I had LVAD removed at } 95 \text { days } \\
\text { and alive at } 18 \text { months; I had LVAD removed at } 50 \text { days and alive at } \\
45 \text { months. } 3 \text { patients died at } 7,9 \text { and II months after insertion of LVAD }\end{array}$ \\
\hline Noda et al., $1989^{110}$ & Survival & $\begin{array}{l}\text { I patient survived and was weaned after } 12 \text { days but died I } 49 \text { days after } \\
\text { removal from infection and cerebral haemorrhage, I patient died of } \\
\text { respiratory failure whilst on the LVAD ( } 12 \text { days) }\end{array}$ \\
\hline \multicolumn{3}{|l|}{ Second-generation LVADs } \\
\hline No studies & & \\
\hline
\end{tabular}

(Table 24 and Appendix 21). ${ }^{107,108}$ Joharchi and colleagues $^{107}$ report survival for one 17 -year-old patient with acute myopericarditis. The patient was successfully weaned from support after 46 days, and was alive 6 months following explantation. Ueno and colleagues ${ }^{108}$ reported survival for a 34-year-old male patient, also with acute myopericarditis. He was successfully weaned after 5 weeks of support, and was alive at 3 months of follow-up.

\section{Functional status}

First-generation devices

None of the studies of the Abiomed BVS 5000, HeartMate, Novacor or Toyobo LVADs reported changes in functional status. ${ }^{104-106,109,110}$ One patient supported with a Thoratec LVAD was reported to be in NYHA Class I at follow-up (6 months) in the case report by Joharchi and colleagues. ${ }^{107}$ This patient had recovered from acute myopericarditis and had been weaned from

\section{Quality of life}

First-generation devices

None of the studies of the Abiomed BVS 5000, HeartMate, Novacor or Toyobo LVADs reported changes in QoL. ${ }^{104-106,109,110}$

\section{Adverse events}

First-generation devices

Abiomed. The case study of the Abiomed BVS

5000 LVAD did not report adverse events. ${ }^{104}$

HeartMate. The one patient supported with a HeartMate LVAD $^{105}$ suffered a drive line infection 3 weeks after device implantation (Table 25 and Appendix 19). This patient, who also had her diagnosis of multiple sclerosis confirmed while on the device, had persistent infection problems with repeated periods of sepsis despite antibiotic treatment until the device was explanted.

Novacor. The case study of the Novacor LVAD did not report adverse events. ${ }^{106}$ 
TABLE 25 Summary of evidence on adverse events following treatment of patients with ESHF as a BTR

\begin{tabular}{|c|c|c|}
\hline Study & Outcomes & Intervention \\
\hline \multicolumn{3}{|l|}{ First-generation LVADs } \\
\hline $\begin{array}{l}\text { HeartMate LVAD } \\
\text { Kjellman et al., 2000 }\end{array}$ & Adverse events & $\begin{array}{l}8 \text { weeks after implantation: relapse of neurological symptoms and } \\
\text { diagnosis of multiple sclerosis confirmed. Infectious problems with } \\
\text { repeated periods of sepsis despite antibiotic treatment, therefore device } \\
\text { was explanted } 83 \text { days after implantation }\end{array}$ \\
\hline \multicolumn{3}{|l|}{ Toyobo LVAD } \\
\hline Nakatani et al., $1998^{109}$ & Adverse events & $\begin{array}{l}\text { I patient LVAS was stopped because of cerebral haemorrhage caused by } \\
\text { infectious aneurysm } 5 \text { months after the start of the LVAS; this patient } \\
\text { died of sepsis } 2 \text { months after discontinuation. } \\
\text { The other two patients developed cerebral embolism following } 3 \text { and } \\
5 \text { months of LVAS support and died at } 9 \text { and I I months from MOF }\end{array}$ \\
\hline Noda et al., 1989110 & $\begin{array}{l}\text { Adverse effects } \\
\text { defined by body } \\
\text { organ not specific } \\
\text { complication }\end{array}$ & $\begin{array}{l}\text { Patient I: massive transfusion before LVAD. Lung and kidney } \\
\text { complications requiring special treatments, }{ }^{a} \text { liver and infection } \\
\text { complications not requiring special treatments } \\
\text { Patient 2: kidney complications requiring special treatments, }{ }^{a} \text { lung, } \\
\text { infection, brain, DIC (not defined by authors, probably disseminated } \\
\text { intravascular coagulation) not requiring special treatments. Special } \\
\text { treatments include high-frequency oscillated ventilation for lung } \\
\text { complication, peritoneal dialysis for kidney failure, plasmapheresis for } \\
\text { liver }\end{array}$ \\
\hline \multicolumn{3}{|l|}{ Second-generation LVADs } \\
\hline No studies & & \\
\hline
\end{tabular}

Toyobo. Adverse events were reported in the two case series of the Toyobo LVAD (Table 25 and Appendix 22). ${ }^{109,110}$ Nakatani and colleagues ${ }^{109}$ noted that three of the five patients died following adverse events. One patient's death was attributed to a cerebral haemorrhage caused by infectious aneurysm 5 months after LVAD implantation, leading to death through sepsis 2 months after discontinuation. The other two patients developed cerebral embolism 3 and 5 months after implantation of the LVAD, which led to MOF. In the case series by Noda and colleagues, ${ }^{110}$ limited details are provided about the complications suffered by the patients. The first patient had lung and kidney complications requiring special treatments, and also liver and infectious complications not requiring special treatments. The second patient had kidney complications requiring special treatments and lung, infection, brain and disseminated intravascular coagulation complications that did not require special treatments. Special treatments were reported to include high-frequency oscillated ventilation for lung complication, peritoneal dialysis for kidney failure and plasmapheresis for liver failure.
Thoratec. The case studies of the Thoratec LVAD did not report any adverse events. ${ }^{107,108}$

\section{Summary of clinical effectiveness of LVADs as a bridge to recovery for ESHF \\ Quantity of evidence}

Seven studies (12 patients) assessed LVADs as a BTR. All studies examined first-generation devices and were either case series or case reports with no comparator intervention.

\section{Methodological quality}

All seven studies were judged to be methodologically weak when assessed against criteria examining rigour and the opportunities for bias.

\section{Generalisability}

The studies included a wide range of patients. Patients were predominantly male $(77 \%)$, aged from 16 to 73 years and suffered from differing underlying conditions including dilated cardiomyopathy (40\%) and acute MI and shock $(23 \%)$. 


\section{Survival}

The case studies for the Abiomed, HeartMate, Novacor and Thoratec LVADs reported that patients were supported with the respective devices for periods ranging from 7 days to 3 months, with all patients recovering to be discharged home and still alive at further followup between 3 months and 1 year later. The case series for the Toyobo LVAD reported survival for seven patients supported from 12 days to 11 months, with two patients alive at 18 and 45 months of follow-up and five patients who died following support for between 12 days and 11 months.

\section{Functional status}

None of the studies of the Abiomed BVS 5000, HeartMate, Novacor or Toyobo LVADs assessed the functional status of patients. One case study of the Thoratec LVAD noted that the patient was in NYHA Class I following support with the LVAD, but provided no baseline assessment.

\section{Quality of life}

None of the studies assessed QoL.

\section{Adverse events}

The case studies of the Abiomed, Novacor and Thoratec devices did not report adverse events. The case study of the HeartMate LVAD reported a drive line infection and repeated periods of sepsis despite antibiotic treatment, until the device was explanted. One of the case series of the Toyobo LVAD reported that three patients died following adverse events including cerebral haemorrhage following an infectious aneurysm and death from sepsis for one patient and cerebral embolism after LVAD implantation and MOF for two patients. The second case series of the Toyobo LVAD reported lung, kidney, liver and infectious complications in the two patients.

\section{Conclusion}

The evidence of the clinical effectiveness of LVADs as a BTR is limited and of poor methodological quality. The systematic review shows that LVADs do appear to provide benefit to patients who would be likely to die from their condition. There was no evidence to judge the effects on the patients' QoL or functional status. Adverse events associated with the devices provide a risk to the patient. Although information was limited from the studies, infections and bleeding associated with the LVADs are the main concern, which when considered with the poor health of the patient may lead to MOF. The studies provided identify whether any of the LVAD was more clinically effective than another LVAD or form of care.

\section{LVADs as a long-term chronic support}

\section{Quantity of research}

Six studies assessing the clinical effectiveness of LVADs as LTCS for patients with ESHF met the inclusion criteria for the systematic review, four studies of first-generation LVADs and two studies of a second-generation LVAD (Table 26 and Appendices 23-27). Of the four studies of firstgeneration devices there was one RCT of the HeartMate VE LVAD, ${ }^{111}$ one series of case reports of the LionHeart LVAD, ${ }^{112}$ one case report of the Novacor LVAD ${ }^{113}$ and one case report of the Toyobo LVAD. ${ }^{114}$ The two studies of the secondgeneration LVAD were a case report ${ }^{94}$ and a case series investigating the use of the Jarvik LVAD for LTCS. ${ }^{95-100}$

\section{Methodological quality of research First-generation LVADs \\ HeartMate}

The methodological quality and the quality of reporting in the study assessing the clinical effectiveness of the HeartMate VE LVAD was judged to be moderate (Table 26 and Appendix 23). ${ }^{111}$ The study, the REMATCH trial, was considered to have a strong study design. It was a multicentre RCT that appeared to use an appropriate method of allocation, randomising patients centrally through a Data and Clinical Coordinating Centre overseen by a clinical 'gatekeeper' with stratification by centre and blocked to try to ensure equal numbers of patients in the arms of the trial. However, as the actual method of randomisation was not described, its method of allocation was rated as moderate. The REMATCH study attempted to select patients that would be representative of those likely to require LTCS using $a$ priori inclusion and exclusion criteria. Modification of the inclusion criteria during recruitment to ensure adequate sample size may have affected the process of selecting a representative sample. As a consequence, selection was judged to be weak. Although it is not clear from the study whether the patients agreed to participate, it is likely given that the study had to adhere to FDA requirements. The study demonstrated that there were no significant differences between the study groups, reducing the risk of confounding. Blinding of patients and the treating surgeons to the interventions compared in 


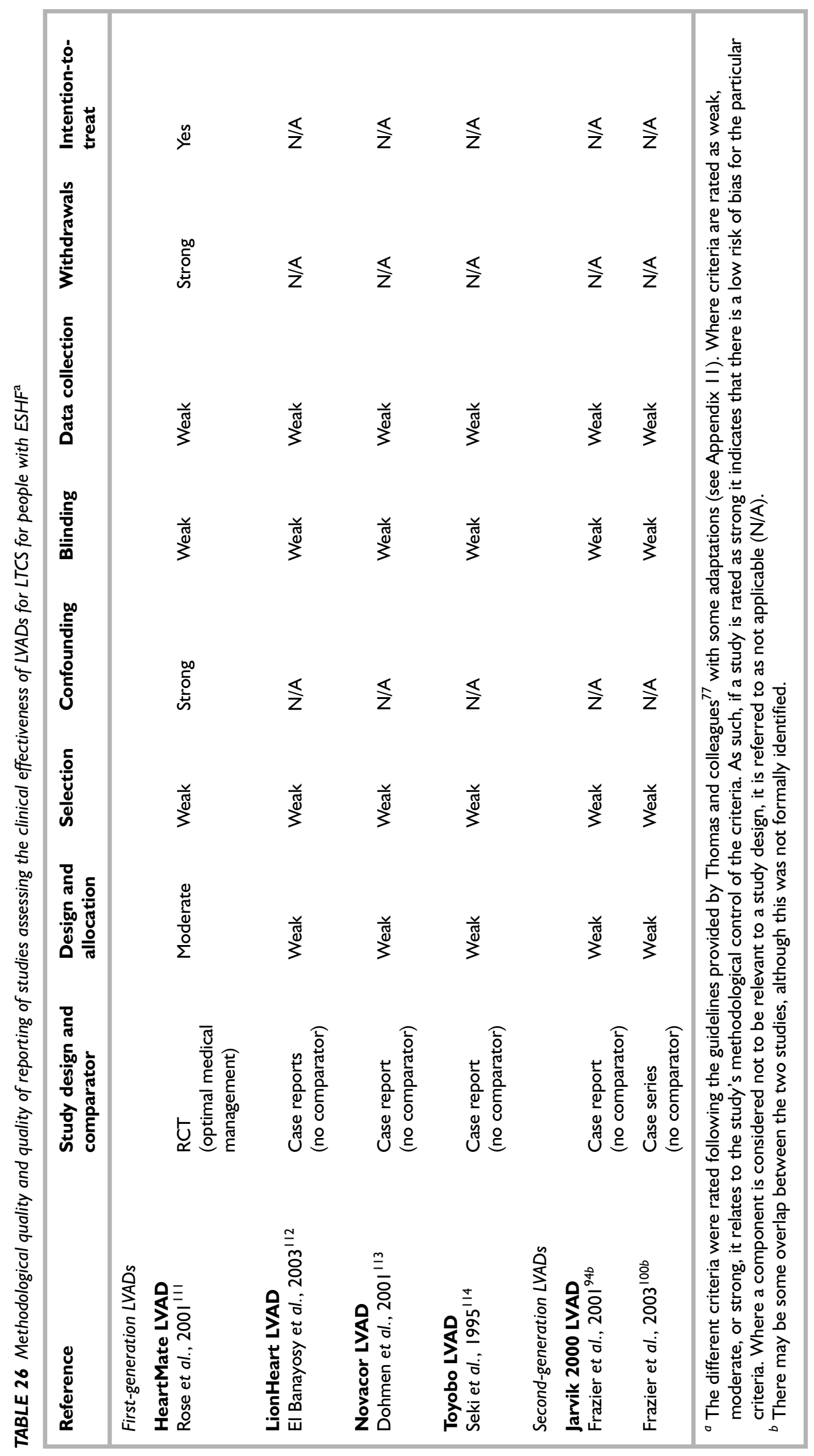


the REMATCH trial was impossible. To try to limit the effects of observational bias, the study used independent blinded observers who had not treated patients to judge outcomes. Although the validity and reliability of the data collection tools received limited discussion, the primary outcome used was death from any cause verified by an independent assessor. Other outcome measures included objective measures of physical functioning using the NYHA classification, 6-minute walk test and maximal oxygen consumption and subjective measures of QoL including the Minnesota Living with Heart Failure questionnaire, the Short Form with Items (SF-36) 36 Health Survey and EuroQoL. The REMATCH trial was judged to be methodologically strong on withdrawals and drop-outs from the study, identifying the numbers of and reasons for withdrawal in addition to undertaking an intention-to-treat analysis.

\section{LionHeart}

The series of case reports of the LionHeart LVAD was judged to be weak in its methodological quality (Table 26 and Appendix 24). ${ }^{112}$ Limited information was provided about the selection of participants, blinding or methods of data collection. As the study was a retrospective series of case reports assessment of confounding, withdrawals and intention-to-treat analyses were considered not to be relevant.

\section{Novacor}

The case report assessing the clinical effectiveness of the Novacor LVAD was judged to be of poor methodological quality (Table 26 and Appendix 25). ${ }^{113}$ The risk of selection bias and observer bias was high. Although objective outcome measures such as survival, NYHA classification and organ function measures were used, limited details were provided on the validity and reliability of the methods of data collection. As a consequence of the study design, assessment of confounding, withdrawals and intention-to-treat analysis were considered not to be relevant.

\section{Toyobo}

The methodological quality of the case report evaluating the Toyobo LVAD was poor (Table 26 and Appendix 26). ${ }^{113}$ Limited details were given in the case report as to how the patients were selected and as to blinding of the assessment of outcomes, providing the possibility for selection and observer bias. Although objective outcome measures such as survival and mortality were used, no details were given of the methods of data collection used and as a consequence it is difficult to determine whether the data collection was valid or reliable. Other quality criteria were considered not to be relevant.

\section{Second-generation LVADs \\ Jarvik}

The case report and case series assessing the Jarvik 2000 LVAD were considered to be weak methodologically (Table 26 and Appendix 27). ${ }^{94-100}$ Although the studies described their selection criteria for participants, its was difficult to judge if the small samples were representative of patients requiring LTCS. Limited information was provided on the methods of data collection and on blinding of outcome assessment. With no comparator group, the quality criteria assessing confounding and withdrawals were of limited applicability.

\section{Generalisability}

The generalisability of the studies was judged on the characteristics of the study participants (Table 27). The REMATCH trial ${ }^{111}$ specified inclusion/exclusion criteria thought to characterise patients requiring LTCS. It was felt likely that the study was representative, characterised by participants who were male (78\% LVADs, $82 \%$ medical), with a mean age between 66 and 68 years, classified as NHYA Class IV, with left ventricular ejection fraction (LVEF) of $17 \%$ and an ischaemic aetiology (LVAD 78\%, medical 69\%). It is more difficult to judge the generalisability of the case reports of the LionHeart, ${ }^{112}$ Novacor $^{113}$ and Toyobo ${ }^{114}$ LVADs and the case series of the Jarvik 2000 LVAD owing to the samples. ${ }^{94-100}$ The series of case reports on the LionHeart LVAD included males aged 55-69 years who suffered from dilated or ischaemic cardiomyopathy, were in NYHA Class IV and were ineligible for heart transplantation. ${ }^{112}$ The study of the Novacor LVAD included a male patient aged 54 years with dilated cardiomyopathy and contraindicated to heart transplantation, while the study of the Toyobo LVAD focused on a male aged 44 years with idiopathic cardiomyopathy. The case report and case series assessing the Jarvik 2000 LVAD included males $(100 \%)$, with a mean age between 61 and 64 years and suffering, predominantly, from dilated cardiomyopathy.

\section{Assessment of the clinical effectiveness of LVADs as a long-term chronic support Survival First-generation LVADs \\ HeartMate. The multicentre REMATCH RCT} assessed the effect on patient survival of the use of the HeartMate VE LVAD $(n=68)$ compared with 


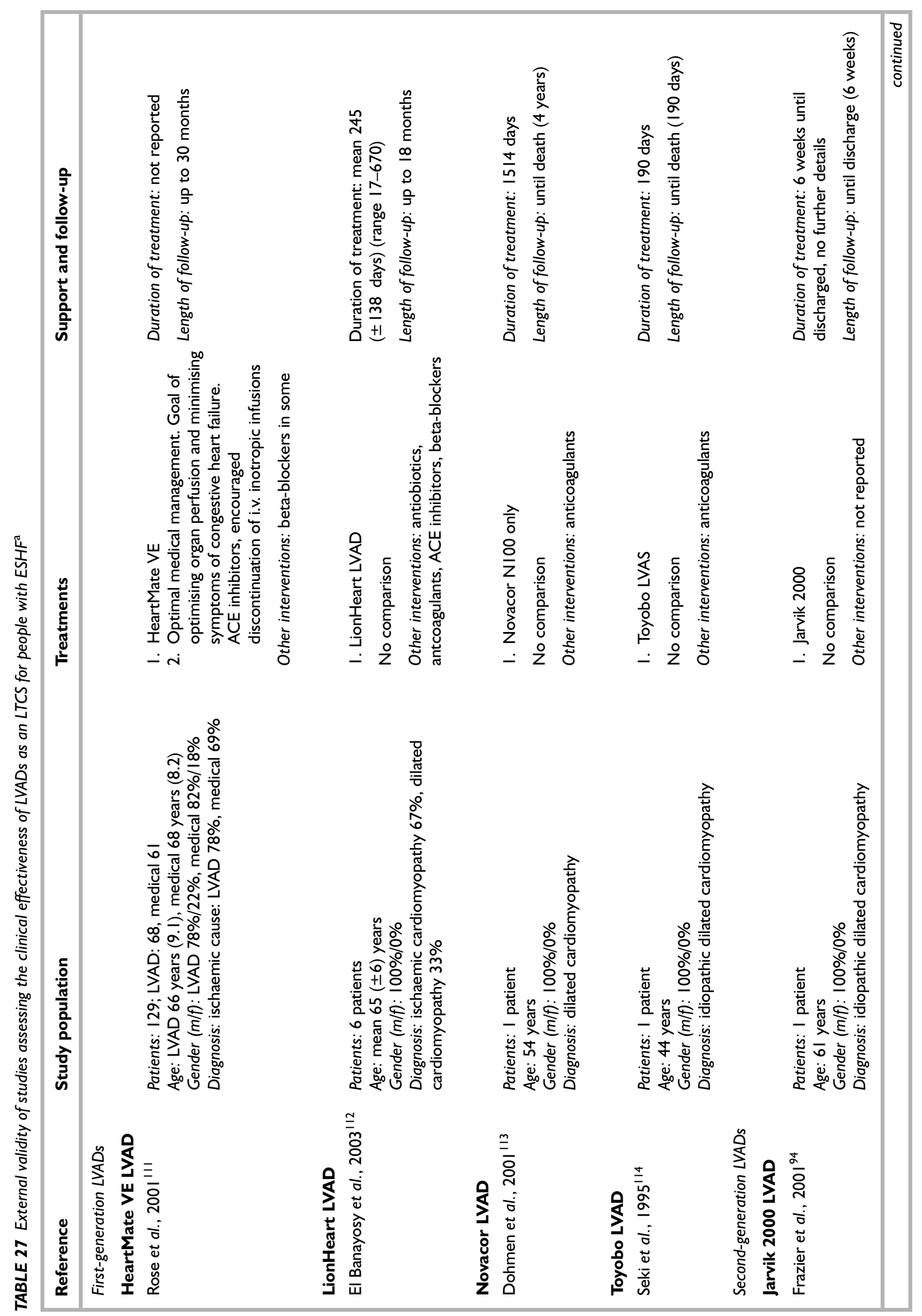




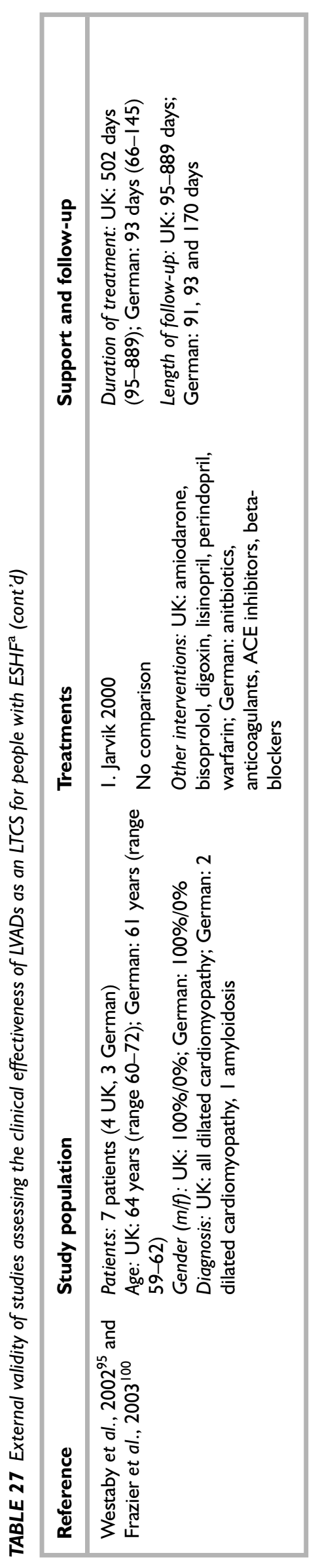


optimal medical management $(n=61)$ (Table 28 and Appendix 23). ${ }^{111}$ Differences were evident in the survival of the groups. Rose and colleagues ${ }^{111}$ reported that there was a $48 \%$ reduction in the risk of death from any cause for those patients receiving the HeartMate VE LVAD compared with those on optimal medical management (RR 0.52; $95 \%$ CI 0.34 to $0.78 ; p=0.001$ ). Actuarial survival rates were significantly higher for those patients receiving a HeartMate VE LVAD compared with those patients on optimal medical treatment at 1 year (52\% versus $25 \%, p=0.002)$ and 2 years (23\% versus $8 \%, p=0.09$ ) follow-up. The difference in survival was reflected in the median length of survival of the groups, with patients in the LVAD group surviving for 408 days compared with 150 days for those in the optimal medical management group. The REMATCH trial assessed the influence of the patient's age on survival undertaking subgroup analysis of 1-year actuarial survival rates in those aged $<60$ years and those aged 60-69 years. One-year survival rates for patients aged $<60$ years were significantly higher $(p=0.05)$ for patients with a HeartMate VE LVAD $(74 \%, n=13)$ than those on optimal medical management $(33 \%, n=9)$. Similarly, patients aged 60-69 years with a HeartMate VE LVAD (47\%, $n=29)$ had significantly $(p=0.009)$ higher 1 -year survival rates than patients receiving optimal medical management $(15 \%, n=20)$.

LionHeart LVAD. One study describing a series of case reports on six patients reported survival following surgery to implant with the LionHeart LVAD and survival at 18 months post-operative follow-up (Table 28 and Appendix 24). ${ }^{12}$ All six patients survived implantation of the LVAD. Three (50\%) of the six patients survived to 18 months follow-up, three died of MOF at 17, 31 and 112 days following implantation of the LVAD.

Novacor. A case study reported survival of one patient supported with a Novacor N100 LVAD (Table 28 and Appendix 25). ${ }^{113,115}$ The 54-year-old male with dilated cardiomyopathy and contraindications for heart transplantation was supported for 1342 days (3.8 years) when the pump was changed. He then survived an additional 172 days to 1514 days of support when he died of causes unrelated to the pump.

Toyobo. A case study reported survival in a 44year-old male with idiopathic dilated cardiomyopathy supported with a Toyobo LVAD (Table 28 and Appendix 26). ${ }^{114}$ The patient survived until 190 days after implantation. No further details were reported.

\section{Second-generation LVADs}

Jarvik. Westaby and colleagues ${ }^{95,96,98,100}$ and Siegenthaler and colleagues ${ }^{99}$ reported survival of seven patients receiving a Jarvik 2000 LVAD as part of a multicentre case series (Table 28 and Appendix 27). Six patients had been discharged from hospital with five continuing to undergo support with the Jarvik 2000 LVAD. Two patients from the UK centre had died after 95 and 382 days. The four patients at the UK centre survived for a mean of 502 days (range 95-889 days) ${ }^{100}$ and the three patients from the German centre for 93 days (range 66-145 days). In a separate case report, Frazier and colleagues ${ }^{94}$ reported that a 61-year-old man survived to discharge 6 weeks post-implantation of the device.

\section{Functional status}

\section{First-generation LVADs}

HeartMate. Functional status was assessed in the REMATCH trial using the NYHA functional classification (Table 29 and Appendix 23). ${ }^{111}$ At baseline assessment Rose and colleagues ${ }^{111}$ found that all patients entering the REMATCH trial were in NYHA Class IV. At the 12-month follow-up patients receiving a HeartMate VE LVAD had improved to NYHA Class II, significantly $(p<0.001)$ better than those patients on optimal medical management who remained in NHYA Class IV. It should be noted that the assessment of NYHA classification at 12 months is limited to a proportion of those patients surviving and able to be assessed (HeartMate VE LVAD 24 of 68 patients; medical management seven of 61 patients).

LionHeart. The series of case reports of the LionHeart LVAD did not assess functional status. ${ }^{112}$

Novacor. The case study of the Novacor LVAD reported that the patient was in NYHA Class I following implantation of the LVAD, but did not provide any baseline assessment (Table 29 and Appendix 25). ${ }^{113,115}$

Toyobo. The case study of the Novacor LVAD did not assess functional status. ${ }^{114}$

\section{Second-generation LVADs}

Jarvik. Westaby and colleagues ${ }^{95,96,98,100}$ and Siegenthaler and colleagues ${ }^{99}$ reported the functional status of seven patients undergoing LTCS with the Jarvik 2000 LVAD (Table 29 and Appendix 27). In the UK study by Westaby and colleagues, ${ }^{95,96,98,100}$ three surviving patients $(n=4)$ had improved from NYHA Class IV at baseline to NYHA Class I (two patients) or II (one patient) within 4 weeks of implantation. 
TABLE 28 Summary of evidence of patient survival following treatment of patients with ESHF as an LTCS

\begin{tabular}{|c|c|c|c|c|}
\hline Study & Outcomes & LVAD & Comparison & $p$-Value \\
\hline \multicolumn{5}{|l|}{ First-generation LVADs } \\
\hline \multicolumn{5}{|l|}{ HeartMate LVAD } \\
\hline \multirow[t]{6}{*}{ Rose et al., 2001 ${ }^{111}$} & Survival at I year (actuarial) & $52 \%$ & $25 \%$ & 0.002 \\
\hline & Survival at 2 years (actuarial) & $23 \%$ & $8 \%$ & 0.09 \\
\hline & Median survival & 408 days & I 50 days & \\
\hline & $\begin{array}{l}\text { Death from any cause } \\
\text { (Kaplan-Meier analysis over } \\
30 \text { months) }\end{array}$ & \multicolumn{3}{|c|}{$\begin{array}{l}\text { Reduction of } 48 \% \text { in the risk of death from any cause in } \\
\text { LVAD group: RR } 0.52(95 \% \mathrm{Cl} 0.34 \text { to } 0.78), p=0.00 \text { I }\end{array}$} \\
\hline & $\begin{array}{l}\text { I-year survival in patients } \\
\text { under } 60 \text { years }(n=22)\end{array}$ & $74 \%(n=13)$ & $33 \%(n=9)$ & 0.05 \\
\hline & $\begin{array}{l}\text { I-year survival inpatients } \\
60-69 \text { years }(n=49)\end{array}$ & $47 \%(n=29)$ & $15 \%(n=20)$ & 0.009 \\
\hline \multirow{3}{*}{$\begin{array}{l}\text { LionHeart LVAD } \\
\text { El Banayosy et al., } \\
2003^{1 / 2}\end{array}$} & & & & \\
\hline & Operative mortality & $\begin{array}{l}100 \% \text { survived } \\
\text { implantation }(n=6)\end{array}$ & N/A & N/A \\
\hline & $\begin{array}{l}\text { Survival at } 18 \text { months } \\
\text { post-implantation }\end{array}$ & $\begin{array}{l}50 \% \text { survived }(n=6) \\
3 \text { patients died at } 17, \\
31 \text { and } 112 \text { days }\end{array}$ & $N / A$ & $N / A$ \\
\hline \multicolumn{5}{|l|}{ Novacor LVAD } \\
\hline $\begin{array}{l}\text { Dohmen et al., } \\
2001,{ }^{113} 1999^{115}\end{array}$ & Survival & $\begin{array}{l}\text { Survived to } 1342 \text { days } \\
\text { ( } 3.8 \text { years) when pump } \\
\text { changed. } \\
\text { Then survived to } \\
\text { I5 I } 4 \text { days until died } \\
\text { of causes unrelated } \\
\text { to the pump }\end{array}$ & $\mathrm{N} / \mathrm{A}$ & N/A \\
\hline \multicolumn{5}{|l|}{ Toyobo LVAD } \\
\hline Seki et al., $1995^{1 / 4}$ & Survival & $\begin{array}{l}\text { Died } 190 \text { days } \\
\text { after surgery }\end{array}$ & $\mathrm{N} / \mathrm{A}$ & $N / A$ \\
\hline \multicolumn{5}{|l|}{ Second-generation LVADs } \\
\hline $\begin{array}{l}\text { Jarvik } 2000 \text { LVAD } \\
\text { Frazier et al., 2001 }\end{array}$ & Survival post-transplant & $\begin{array}{l}\text { I patient survived for } \\
6 \text { weeks until } \\
\text { discharged, no } \\
\text { further details }\end{array}$ & N/A & $\mathrm{N} / \mathrm{A}$ \\
\hline $\begin{array}{l}\text { Westaby et al., } 2002^{95} \\
\text { and Frazier et al., } \\
2003^{100}\end{array}$ & Survival & $\begin{array}{l}\text { UK study }(n=4) \text { : } \\
3 \text { patients left hospital } \\
\text { within } 4 \text { weeks (one } \\
\text { report states that } \\
\text { three patients left } \\
\text { hospital within } \\
3-8 \text { weeks), } 2 \text { patients } \\
\text { died at } 95 \text { and } \\
382 \text { days. The } \\
\text { surviving } 2 \text { patients } \\
\text { were still supported } \\
\text { after } 642 \text { and } 889 \text { days. } \\
\text { German study ( } n=3 \text { ): } \\
3 \text { patients surviving } \\
\text { at follow-up }\end{array}$ & $\mathrm{N} / \mathrm{A}$ & $N / A$ \\
\hline
\end{tabular}


TABLE 29 Summary of evidence of patient functional status following treatment of patients with ESHF as an LTCS

\begin{tabular}{|c|c|c|c|c|}
\hline Study & Outcomes & Intervention & Comparison & $p$-Value \\
\hline \multicolumn{5}{|l|}{ First-generation LVADs } \\
\hline $\begin{array}{l}\text { HeartMate LVAD } \\
\text { Rose et al., 2001 }\end{array}$ & Median NYHA class & $\begin{array}{l}\text { No. assessed: } 24 / 24 \\
\text { (I00\%) } \\
\text { Score: II }\end{array}$ & $\begin{array}{l}\text { No. assessed: } 7 / 1 \mathrm{I} \\
(64 \%) \\
\text { Score: IV }\end{array}$ & $<0.001$ \\
\hline $\begin{array}{l}\text { LionHeart LVAD } \\
\text { El Banayosy et al., } \\
2003^{1 / 2}\end{array}$ & Not assessed & & & \\
\hline $\begin{array}{l}\text { Novacor LVAD } \\
\text { Dohmen et al., } \\
2001,{ }^{13} \text { 1999'15 }\end{array}$ & Not assessed at baseline & & & \\
\hline $\begin{array}{l}\text { Toyobo LVAD } \\
\text { Seki et al., } 1995^{1 / 4}\end{array}$ & Not assessed & & & \\
\hline \multicolumn{5}{|l|}{ Second-generation LVADs } \\
\hline \multicolumn{4}{|l|}{ Jarvik 2000 LVAD } & \\
\hline \multirow[t]{3}{*}{ Westaby et al., $2002^{95}$} & $\begin{array}{l}\text { NYHA functional } \\
\text { classification }\end{array}$ & & & \\
\hline & Baseline $(n=3)$ & $3 / 3$ in Class IV & No comparator & \\
\hline & $\begin{array}{l}\text { Post-implantation of LVAD } \\
\text { (4 weeks): }\end{array}$ & $\begin{array}{l}2 / 3 \text { in Class I } \\
I / 3 \text { in Class II }\end{array}$ & No comparator & \\
\hline
\end{tabular}

A subsequent update report by Frazier and colleagues ${ }^{100}$ stated that one patient subsequently died. Siegenthaler and colleagues ${ }^{99}$ noted that all three patients were classified as NYHA Class I or II at follow-up (range 14-93 days postoperatively), but provided no baseline assessment. Frazier and colleagues ${ }^{94}$ did not assess functional status. ${ }^{94}$

\section{Quality of life}

\section{First-generation LVADs}

HeartMate. The REMATCH trial ${ }^{111}$ reported the QoL of patients with the HeartMate LVAD compared with those on optimal medical management at baseline and at 12 months' followup on a range of measures (Table 30 and Appendix 23). All patients entering the RCT completed the baseline assessment on Minnesota Living with Heart Failure score, the physical function and emotional role subscales of the SF-36 and on the Beck Depression Inventory, with no significant difference between the groups. At the 12-month follow-up only a limited proportion of the patients included at allocation to treatment groups had survived and were able to complete the assessments of QoL. On the Minnesota Living with Heart Failure score (total score 0-105, higher score equates to worse QoL) patients with the
HeartMate VE LVAD [mean 41 (SD 22), $n=23$ ] had better QoL at 12 months than those patients receiving medical management [mean 58 (SD 21), $n=6]$, although the difference was not statistically significant $(p=0.11)$. Comparison on the SF-36 [physical function and emotional role, scored 0 (worst) to 100 (best)] showed that patients receiving the HeartMate VE LVAD had significantly better QoL on the physical function [mean 46 (SD 19), $n=23$ versus mean 21 (SD 21), $n=6 ; p=0.01$ ] and emotional role [mean 64 (SD 45), $n=23$ versus mean 17 (SD 28), $n=6$; $p=0.03$ ] subscales than patients on optimal medical management. Depression was assessed on the Beck Depression Inventory (score 0-9 normal, 10-18 mild to moderate depression, 19-29 moderate to severe depression, 30-64 severe depression). Patients on optimal medical management suffered from significantly $(p=0.04)$ worse depression than those with a HeartMate VE LVAD [13 (SD 7), $n=22$ versus 8 (SD 7), $n=5$ ].

LionHeart. The series of case reports of the LionHeart LVAD did not assess QoL. ${ }^{112}$

Novacor. The case study of the Novacor LVAD did not assess QoL. ${ }^{113,115}$ 
TABLE 30 Summary of evidence of QoL following treatment of patients with ESHF as an LTCS

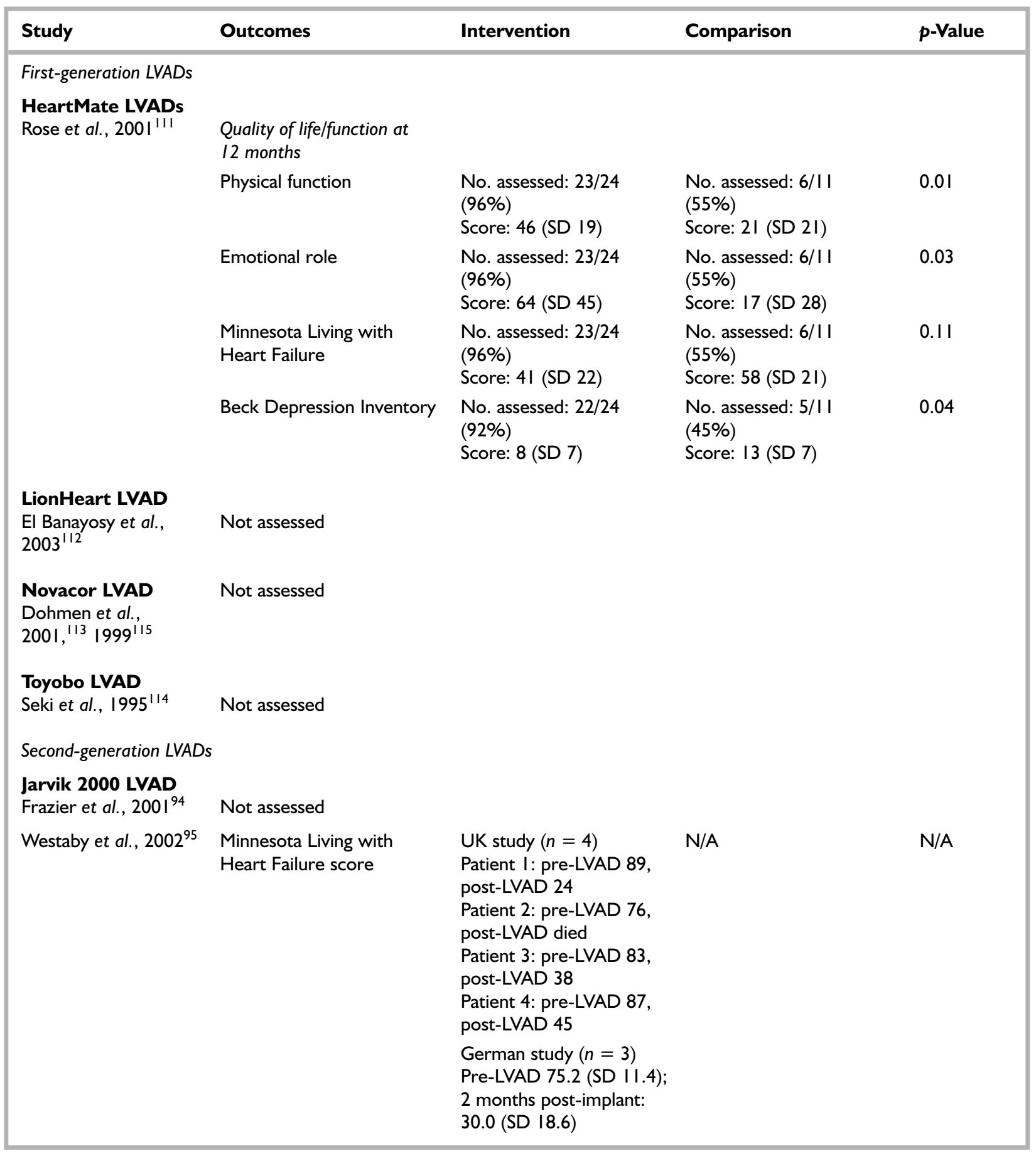

Toyobo. The case study of the Novacor LVAD did not assess QoL. ${ }^{114}$

\section{Second-generation LVADs}

Jarvik. The multicentre case series of the Jarvik 2000 LVAD $^{95,96,98,100}$ reported the QoL of seven patients undergoing LTCS (Table 30 and Appendix 27). In the UK, Westaby and colleagues $^{98}$ noted that the QoL improved for the with Heart Failure Questionnaire following implantation of the LVAD. An updated report by Frazier and colleagues ${ }^{100}$ does not provide additional information on the long-term QoL of these patients other than to report that one of the three patients died and one of the remaining two long-term survivors was travelling extensively and participating in physical challenges. Siegenthaler and colleagues ${ }^{99}$ reported that the mean QoL score for the three patients at the German centre 
improved from the preoperative score of 75.2 (SD 11.4) to the postoperative 2-month score of 30.0 (SD 18.6). Frazier and colleagues ${ }^{94}$ did not assess QoL. ${ }^{94}$

\section{Adverse events}

\section{First-generation devices}

HeartMate. Rose and colleagues ${ }^{111}$ compared the incidence of serious adverse events in the REMATCH trial for the patients in the HeartMate VE LVAD group and the medical therapy group, in addition to reporting serious adverse events related specifically to the LVAD (Table 31 and Appendix 23). These were reported as event rates per patient-year with comparison through the rate ratio (95\% CIs). Patients receiving a HeartMate VE LVAD were more than twice as likely to suffer a serious adverse event (an accumulation of all adverse events reported) than patients receiving medical therapy [rate ratio: 2.35 (95\% CI 1.86 to 2.95]. Of the specific adverse events reported, patients with a HeartMate VE LVAD were significantly more likely to suffer from nonneurological bleeding [rate ratio 9.47 (95\% CI 2.30 to 38.90$)$ ] or neurological dysfunction (includes stroke, transient ischaemic attacks and toxic or metabolic encephalopathy) [rate ratio 4.35 (95\% CI 1.31 to 14.50$)$ ] than patients receiving medical therapy. Patients with an LVAD suffered higher rates of supraventricular arrhythmias, peripheral embolic events, sepsis, local infection, renal failure, miscellaneous adverse events, syncope and serious psychiatric disease than patients receiving medical therapy, although the differences were not statistically significant. In contrast, patients on medical therapy reported significantly higher rates of ventricular arrhythmias than patients with an LVAD [rate ratio 0.45 (95\% CI 0.22 to 0.90$)$ ]. Also, patients on medical therapy incurred higher rates of cardiac arrest and non-perioperative MI than patients with an LVAD. The serious adverse events related specifically to the use of an LVAD included: suspected malfunction of LVAD (rate per patientyear 0.75$)$; perioperative bleeding $(0.46)$; infection of the drive-line tract or pocket $(0.41)$; infection of pump interior, inflow tract or outflow tract (0.23); right heart failure (0.17); failure of LVAD system (0.08); and thrombosis in LVAD (0.06). Rose and colleagues ${ }^{111}$ reported the cause of death of patients during the REMATCH trial. Of the 41 patients with an LVAD who died, 17 were due to sepsis, seven to LVAD failure, five to miscellaneous noncardiovascular causes and four to

cerebrovascular disease. In contrast, 50 of the 54 deaths of patients in the medical therapy group were due to left ventricular dysfunction.
LionHeart. El Banayosy and colleagues ${ }^{112}$ reported adverse events for six patients who received the LionHeart LVAD (Table 31 and Appendix 24). Of the six patients, three suffered from temporary haemolysis, three reported bleeding and two had an early arrhythmia. Other adverse events included reoperation for bleeding (one patient), tamponade (one patient), gastrointestinal ischaemia (one patient), outflow graft kink (one patient), low pump output (one patient), cerebrovascular accident (one patient) and controller change (one patient). In addition, the three surviving patients were readmitted to hospital on three occasions for urinary tract infection, renal calculi and battery change (one patient), controller change (one patient) and spontaneous bleeding from femoral haematoma and late haemolysis after 6 months (one patient). There were no admissions for infection, thromboembolic complications or arrhythmias.

Novacor. In the case study of the Novacor device, ${ }^{113,115}$ the patient suffered from transient renal failure during the postoperative period (Table 31 and Appendix 25). In addition, the recovery of the patient was complicated by bronchopneumonia and the need for a tracheostomy due to prolonged ventilation. Once discharged home on support, the patient suffered from febrile episodes that were traced to an infection of the inflow and outflow valve conduits, which were then replaced.

Toyobo. Adverse effects were reported in the one patient being supported by the Toyobo LVAD (Table 31 and Appendix 26). ${ }^{114}$ A cerebral embolism which resulted in a hemiparesis was noted on the ninth postoperative day, and following this the patient suffered from multiple cerebral embolisms on the 57 th and 175 th postoperative days. These led to a left hemiplegia, aphasia and loss of consciousness. The pump's electromagnetic valve was exchanged at day 91, and the blood pump was exchanged on the 141st postoperative day owing to thrombi attaching to the pump surface.

\section{Second-generation LVADs}

Jarvik. Westaby and colleagues ${ }^{95,96,98}$ reported that one patient suffered dyspnoea at 4 months, one patient had ventricular tachycardia at 11 months, one patient experienced three power supply problems with the LVAD and one patient suffered an infection following a blood transfusion (Table 31 and Appendix 27). It was noted that none of the three surviving patients suffered from infection of the skull-mounted percutaneous 
TABLE 3 I Summary of evidence on adverse events following treatment of patients with ESHF as an LTCS

\begin{tabular}{|c|c|c|c|c|}
\hline Study & Outcome & Intervention & Comparison & $p$-Value \\
\hline \multicolumn{5}{|l|}{ First-generation LVADs } \\
\hline \multirow{24}{*}{$\begin{array}{l}\text { HeartMate LVAD } \\
\text { Rose et al., 200 I }\end{array}$} & & & & \\
\hline & & & & \\
\hline & Cause of death & & & \\
\hline & Left ventricular dysfunction & I (I\%) & $50(82 \%)$ & \\
\hline & Sepsis & $\begin{array}{l}|7 / 4|(4 \mid \% \text { of } \\
\text { deaths) }\end{array}$ & I (2\%) & \\
\hline & Failure of LVAD & $\begin{array}{l}7 / 4 I(17 \% \text { of } \\
\text { deaths) }\end{array}$ & 0 & \\
\hline & $\begin{array}{l}\text { Miscellaneous non-cardiovascular } \\
\text { causes }\end{array}$ & $5(7 \%)$ & 0 & \\
\hline & Cerebrovascular disease & $4(6 \%)$ & 0 & \\
\hline & $\begin{array}{l}\text { Miscellaneous cardiovascular } \\
\text { causes }\end{array}$ & $2(3 \%)$ & I (2\%) & \\
\hline & Pulmonary embolism & $2(3 \%)$ & 0 & \\
\hline & Acute MI & 0 & I (2\%) & \\
\hline & Cardiac procedure & 0 & I (2\%) & \\
\hline & Perioperative bleeding & I (I\%) & 0 & \\
\hline & Unknown & $2(3 \%)$ & 0 & \\
\hline & $\begin{array}{l}\text { Incidence of serious adverse } \\
\text { events (rate/patient-year) }\end{array}$ & $n=60$ & $n=67$ & \\
\hline & Any serious adverse event & 6.45 & 2.75 & $\begin{array}{l}\text { Rate ratio } 2.35 \\
(95 \% \mathrm{Cl} 1.86 \text { to } \\
2.95)\end{array}$ \\
\hline & Non-neurological bleeding & 0.56 & 0.06 & $\begin{array}{l}\text { Rate ratio } 9.47 \\
(95 \% \mathrm{Cl} 2.30 \text { to } \\
38.90)\end{array}$ \\
\hline & $\begin{array}{l}\text { Neurological dysfunction } \\
\text { (stroke, transient ischaemic } \\
\text { attack, toxic or metabolic } \\
\text { encephalopathy) }\end{array}$ & 0.39 & 0.09 & $\begin{array}{l}\text { Rate ratio } 4.35 \\
(95 \% \mathrm{Cl} \mathrm{I.3I} \mathrm{to} \\
14.50)\end{array}$ \\
\hline & Supraventricular arrhythmia & 0.12 & 0.03 & $\begin{array}{l}\text { Rate ratio } 3.92 \\
(95 \% \mathrm{Cl} 0.47 \text { to } \\
32.40)\end{array}$ \\
\hline & Sepsis & 0.60 & 0.30 & $\begin{array}{l}\text { Rate ratio } 2.03 \\
(95 \% \mathrm{Cl} 0.99 \text { to } \\
4.13)\end{array}$ \\
\hline & Local infection & 0.39 & 0.24 & $\begin{array}{l}\text { Rate ratio } 1.63 \\
(95 \% \mathrm{Cl} 0.72 \text { to } \\
3.70)\end{array}$ \\
\hline & Renal failure & 0.25 & 0.18 & $\begin{array}{l}\text { Rate ratio } \mathrm{I} .42 \\
(95 \% \mathrm{Cl} 0.54 \text { to } \\
3.7 \mathrm{I})\end{array}$ \\
\hline & Miscellaneous adverse events & 1.37 & 0.98 & $\begin{array}{l}\text { Rate ratio } 1.4 \mathrm{I} \\
(95 \% \mathrm{Cl} 0.93 \text { to } \\
2.12)\end{array}$ \\
\hline & Syncope & 0.04 & 0.03 & $\begin{array}{l}\text { Rate ratio } 1.31 \\
(95 \% \mathrm{Cl} 0.12 \text { to } \\
14.40)\end{array}$ \\
\hline
\end{tabular}


TABLE 3 I Summary of evidence on adverse events following treatment of patients with ESHF as an LTCS (cont'd)

\begin{tabular}{|c|c|c|c|c|}
\hline Study & Outcome & Intervention & Comparison & $p$-Value \\
\hline & Serious psychiatric disease & 0.04 & 0.03 & $\begin{array}{l}\text { Rate ratio I.3I } \\
(95 \% \mathrm{Cl} 0.12 \text { to } \\
14.30)\end{array}$ \\
\hline & Cardiac arrest & 0.12 & 0.18 & $\begin{array}{l}\text { Rate ratio } 0.65 \\
(95 \% \mathrm{Cl} 0.21 \text { to } \\
2.00)\end{array}$ \\
\hline & Non-perioperative MI & 0.02 & 0.03 & $\begin{array}{l}\text { Rate ratio } 0.65 \\
(95 \% \mathrm{Cl} 0.04 \text { to } \\
10.30)\end{array}$ \\
\hline & Ventricular arrhythmia & 0.25 & 0.56 & $\begin{array}{l}\text { Rate ratio } 0.45 \\
(95 \% \mathrm{Cl} 0.22 \text { to } \\
0.90)\end{array}$ \\
\hline & Hepatic failure & 0.02 & 0.0 & \\
\hline & $\begin{array}{l}\text { LVAD-related events } \\
\text { (rate/patient year) }\end{array}$ & & & \\
\hline & Suspected malfunction of LVAD & 0.75 & & \\
\hline & Perioperative bleeding & 0.46 & & \\
\hline & $\begin{array}{l}\text { Infection of drive-line tract or } \\
\text { pocket }\end{array}$ & 0.41 & & \\
\hline & $\begin{array}{l}\text { Infection of pump interior, } \\
\text { inflow tract or outflow tract }\end{array}$ & 0.23 & & \\
\hline & Right heart failure & 0.17 & & \\
\hline & Failure of LVAD system & 0.08 & & \\
\hline & Thrombosis in LVAD & 0.06 & & \\
\hline & Perioperative $\mathrm{MI}$ & 0.0 & & \\
\hline \multirow[t]{16}{*}{$\begin{array}{l}\text { LionHeart LVAD } \\
\text { El Banayosy et al., } 2003^{1 / 2}\end{array}$} & \multicolumn{2}{|l|}{$\begin{array}{l}\text { Patients suffering from adverse } \\
\text { events }(n=6)\end{array}$} & No comparator & \\
\hline & Haemolysis (temporary) & 3 patients & & \\
\hline & Bleeding & 3 patients & & \\
\hline & Early arrhythmia & 2 patients & & \\
\hline & Reoperation for bleeding & I patient & & \\
\hline & Tamponade & I patient & & \\
\hline & Gastrointestinal ischaemia & I patient & & \\
\hline & Outflow graft kink & I patient & & \\
\hline & Low pump output & I patient & & \\
\hline & Cerebrovascular accident & I patient & & \\
\hline & Controller change & I patient & & \\
\hline & Readmission to hospital & & & \\
\hline & $\begin{array}{l}\text { Urinary tract infection, renal } \\
\text { calculi and battery change }\end{array}$ & I patient & & \\
\hline & Controller change & I patient & & \\
\hline & $\begin{array}{l}\text { Spontaneous bleeding from } \\
\text { femoral haematoma and late } \\
\text { haemolysis after } 6 \text { months }\end{array}$ & I patient & & \\
\hline & $\begin{array}{l}\text { Readmissions for infection, } \\
\text { thromboembolic complications } \\
\text { or arrhythmias }\end{array}$ & none & & \\
\hline
\end{tabular}


TABLE 3 I Summary of evidence on adverse events following treatment of patients with ESHF as an LTCS (cont'd)

\begin{tabular}{|c|c|c|c|}
\hline Study & Intervention & Comparison & $p$-Value \\
\hline $\begin{array}{l}\text { Novacor LVAD } \\
\text { Dohmen et al., 200I, }{ }^{13} \\
\text { 1999115 }\end{array}$ & $\begin{array}{l}\text { Postoperative period complicated } \\
\text { by transient renal failure } \\
\text { Recovery complicated by } \\
\text { bronchopneumonia } \\
\text { Tracheostomy required due to } \\
\text { prolonged ventilation } \\
\text { Febrile episodes while at home, } \\
\text { traced to Staphylococcus aureus } \\
\text { infection of the inflow and } \\
\text { outflow valve conduits (replaced) }\end{array}$ & No comparator & \\
\hline \multicolumn{4}{|l|}{ Toyobo LVAD } \\
\hline Seki et al., $1995^{114}$ & $\begin{array}{l}\text { On ninth postoperative day patient } \\
\text { had cerebral embolism resulting in } \\
\text { hemiparesis. Multiple } \\
\text { cerebralembolisms on } 57 \text { th and } \\
\text { I75th postoperative days. Developed } \\
\text { left hemiplegia, aphasia and loss of } \\
\text { consciousness. Died I } 90 \text { days after } \\
\text { surgery. No other major complications } \\
\text { such as infection, bleeding, hepatic } \\
\text { or renal dysfunction. Electromagnetic } \\
\text { valve exchanged at day } 91 \text {. Blood } \\
\text { pump exchanged on } 141 \text { st } \\
\text { postoperative day owing to thrombi } \\
\text { attaching to pump surface }\end{array}$ & No comparator & \\
\hline \multicolumn{4}{|l|}{ Second-generation LVADs } \\
\hline \multicolumn{4}{|l|}{ Jarvik 2000 LVAD } \\
\hline \multirow[t]{11}{*}{ Westaby et al., $2002^{95}$} & Adverse events & No comparator & \\
\hline & UK study $(n=4)$ & & \\
\hline & $\begin{array}{l}\text { Significant haemolysis } 0 \text {; dyspnoea } \\
\text { I patient at } 4 \text { months; VT I patient } \\
\text { at II months; thrombus } 0\end{array}$ & & \\
\hline & $\begin{array}{l}\text { Device-related complications } \\
\text { I patient suffered } 3 \text { power supply } \\
\text { problems; I patient suffered an } \\
\text { infection from a blood transfusion }\end{array}$ & & \\
\hline & $\begin{array}{l}\text { German study }(n=3) \\
\text { Transient ischaemic attack I; ventricular } \\
\text { arrythmia I }\end{array}$ & & \\
\hline & Minor events: & & \\
\hline & $\begin{array}{l}\text { I episode of loss of consciousness while } \\
\text { battery changed }\end{array}$ & & \\
\hline & $\begin{array}{l}\text { I knee effusion after vigorous ergometry } \\
\text { training }\end{array}$ & & \\
\hline & I large skin abrasion from adhesive tape & & \\
\hline & I hospital readmission due to dehydration & & \\
\hline & $\begin{array}{l}2 \text { patients required postoperative } \\
\text { psychological therapy }\end{array}$ & & \\
\hline
\end{tabular}


power delivery system. A further update by Frazier and colleagues ${ }^{100}$ provided no additional information on adverse events, although it was noted that patients received serious patient-related complications including postoperative bleeding, left ventricular thrombus, coronary thrombosis, subdural haematoma and gastrointestinal bleeding. There were no system failures but there were technical difficulties with batteries and connectors. Siegenthaler and colleagues ${ }^{99}$ reported no infections, device-related complications or reoperations. Minor adverse events were reported. Frazier and colleagues ${ }^{94} \mathrm{did}$ not assess adverse events suffered by the patient undergoing LTCS.

\section{Summary of clinical effectiveness for LVADs as a long-term chronic support for ESHF Quantity of evidence}

Six studies assessed the clinical effectiveness of LVADs as an LTCS, with four studies of the HeartMate, LionHeart, Novacor and Toyobo firstgeneration LVADs and two of the Jarvik 2000 second-generation LVAD.

\section{Methodological quality}

The methodological quality of the studies varied. Only the RCT of the HeartMate LVAD was judged to be moderate to strong in its methodological rigour. The studies of the other devices were observational in design and were considered to be weak in the methodology adopted in terms of preventing opportunities for bias.

\section{Generalisability}

The RCT of the HeartMate LVAD was felt to represent those most likely to benefit from LTCS. Patients were predominantly male $(\sim 80 \%)$, aged over 65 years, with an ischaemic underlying cause (70-80\%) and contraindicated for heart transplantation. The case series of the Jarvik 2000 was also thought to be fairly representative of those who may benefit, including males aged 59-72 years, suffering predominantly from dilated cardiomyopathy. The other studies of the Jarvik, LionHeart, Novacor and Toyobo devices included male patients, aged 44-69 years and suffering dilated or ischaemic cardiomyopathy.

\section{Survival}

The HeartMate LVAD was shown to lead to a statistically significant $48 \%$ reduction in the risk of death from any cause compared with optimal medical management. Actuarial survival was significantly higher for patients with the HeartMate LVAD compared with optimal medical management at 1 year (52\% versus $25 \%)$ and 2 years (23\% versus $8 \%$ ) of follow-up. The significant difference in 1-year survival was evident for patients aged $<60$ years and those aged 60-69 years. The case reports of the LionHeart LVAD found that $50 \%$ of patients survived to 18 months of follow-up. The case reports of the Novacor and the Toyobo LVADs showed patients were supported for over 4 years and 190 days, respectively. The multicentre case series of seven patients with the Jarvik 2000 LVAD showed that six patients have survived for between 66 to 455 days of support, with one patient dying after 94 days. A case study of the Jarvik 2000 LVAD reported survival to discharge of a patient at 6 weeks post-implantation.

\section{Functional status}

The functional status of a subset of patients receiving HeartMate LVAD and who were well enough to be assessed improved significantly from NYHA Class IV at baseline to NYHA Class II at 12 months of follow-up compared with those patients on optimal medical management who remained in NYHA Class IV. The functional status of patients receiving the Jarvik 2000 LVAD improved from NYHA Class IV to either Class I or II following implantation. Functional status was not assessed at baseline and follow-up for the LionHeart, Novacor and Toyobo LVADs.

\section{Quality of life}

QoL was not assessed for the LionHeart, Novacor and Toyobo LVADs. The RCT comparing the HeartMate LVAD with optimal medical management found that QoL for those with the LVAD improved compared with those on optimal medical management on a range of measures, significantly for SF-36 subscales on physical function and emotional role and on the Beck Depression Inventory. The case series of the Jarvik 2000 LVAD showed that patients' QoL on the Minnesota Living with Heart Failure Questionnaire improved following implantation of the device.

\section{Adverse events}

Patients receiving a HeartMate LVAD were twice as likely to suffer a serious adverse event than those on optimal medical management, with significantly higher rates of non-neurological bleeding and neurological dysfunction. In addition, patients with a HeartMate LVAD had higher rates of supraventricular arrhythmias, peripheral embolic events, sepsis, local infection, renal failure, miscellaneous adverse events, syncope and serious psychiatric disease than 
patients receiving medical therapy. Patients on optimal medical therapy had higher rates of ventricular arrhythmias, cardiac arrest and nonperioperative MI. Of the HeartMate patients who died, $41 \%$ were due to sepsis, $17 \%$ to LVAD failure and $10 \%$ to cerebrovascular disease, whereas $93 \%$ of optimal medical management patients died from left ventricular dysfunction. The case study of the Novacor device found that the patient suffered from transient renal failure, bronchopneumonia and febrile episodes due to device-related infection. Patients with the LionHeart LVAD suffered temporary haemolysis (50\%), bleeding (50\%), early arrhythmia $(40 \%)$ and other adverse events. It was reported that no patients with the LionHeart were admitted with infection, thromboembolic complications or arrhythmias. With the Toyobo LVAD the patient suffered from multiple cerebral embolisms and thrombi within the pump. The case series of the Jarvik 2000 reported cases of dyspnoea,
VT, power supply problems and infection. No other device-related malfunctions or infections were reported.

\section{Conclusion}

There is good-quality evidence that LVADs provide benefit to patients as a long-term support,

lengthening the patient's life and its quality when compared with optimal medical therapy. Adverse events associated with the use of LVADs include device malfunctions, infection and bleeding associated with their use. Evidence of the clinical effectiveness of the different devices indicates that the HeartMate LVAD appears to be effective when compared with optimal medical management. For the second-generation devices, the early evidence suggests that the Jarvik 2000 shows promise, but further research is needed to assess whether there will be any long-term consequences associated with the change in the nature of the circulatory support. 


\section{Chapter 4}

\section{Systematic review of the costs and cost-effectiveness of LVADs for people with ESHF}

\section{Introduction}

A systematic review was conducted to identify economic studies or costing papers evaluating the use of LVADs for people with ESHF for the three indications of BTT, BTR and LTCS. For each indication, the quantity and quality of research evidence available for the different LVADs are discussed and the outcomes assessed. This detailed approach was undertaken for all studies which included a cost analysis. In contrast to the systematic review of clinical effectiveness, this review did not restrict the assessment to specific LVADs. This approach was taken owing to the apparent scarcity of evidence on the costeffectiveness of this technology and the need for data to develop and populate an economic evaluation for the UK. Although such an approach involves some uncertainty about the appropriateness of such data, its effects may be evaluated through sensitivity analyses. For the purpose of this systematic review, the economic studies retrieved were placed into four groupings: cost summation; cost-minimisation analysis; costeffectiveness analysis and cost-utility analysis. Studies which reported utilities for patients with LVADs or with ESHF were reviewed with the aim of populating an economic model for the UK. Full details of the research methods for the systematic review are discussed in Chapter 2.

\section{Quantity of research}

Nineteen studies were identified which evaluated the costs or cost-effectiveness of LVADs for people with ESHF. In 15 studies the LVAD studied was identified as a first-generation LVAD. Although the remaining four studies did not specify the LVAD used, it is likely that they were first-generation devices. A summary of the key characteristics of these studies, in terms of country, device, indication, sample size and method of economic analysis, is provided in Table 32 with additional details provided in Appendices 28 and 29.

The majority of published research in the area constituted simple cost summation or cost minimisation analyses conducted in poorly controlled samples of LVAD patients, primarily based in the USA. A number of limitations apply to the usefulness of such data outside its specific context. First, the generalisability of cost data from one healthcare system to another is very limited. ${ }^{116}$ This is of particular concern in the USA where the majority of 'costs' are presented in the form of insurance charges which are fixed reimbursements and do not specify the actual amount of resource used for a given centre. Second, US research must be viewed in the light of the strict FDA regulations, which were initially in place in the USA with regard to the treatment protocols surrounding the use of LVADs. Prior to 1995, LVADs were restricted to inpatient use for BTT. Thereafter some limited outpatient use was indicated. ${ }^{117}$ Following a further update of FDA regulations in 1998, there has been increasing opportunity for LVAD support to be managed on an outpatient basis, and in 2002 LVADs were licensed for use as a permanent alternative to transplantation (referred to as LTCS in this study). ${ }^{118}$ Costs collected in the different time periods are therefore not comparable owing to the increased costs associated with FDA-mandated length of stay (LOS). To combat these problems, focus was directed on papers where resource use was presented in natural units (e.g. number of follow-up appointments) alongside estimates of cost.

Three studies were identified eliciting utilities. One was specific to LVADs, ${ }^{138}$ with the remaining two reporting utilities for ESHF patients ${ }^{139,140}$ The studies are summarised in Table 33. A fuller discussion of these studies is presented in the section 'Cost-utility analyses', p. 80.

\section{Methodological quality of research}

The methodological quality and quality of reporting of the studies was assessed using an adapted version of the criteria outlined by Drummond and Jefferson. ${ }^{116}$ Although all studies were assessed, only the quality of those reported as full papers are discussed in detail as those 
TABLE 32 Summary of studies of costs and cost-effectiveness of LVADs for people with ESHF

\begin{tabular}{|c|c|c|c|c|c|}
\hline $\begin{array}{l}\text { Study (listed alphabetically } \\
\text { with abstracts at the end) }\end{array}$ & Country & Device & Indication & Type of analysis & Sample size \\
\hline \multicolumn{6}{|l|}{ Full studies } \\
\hline Arabia et al., $1996^{119}$ & USA & Novacor & BTT & CMA & 4 \\
\hline CETS, $2000^{120}$ & Canada & Novacor & BTT/LTCS & CEA: cost per LYG & Modelled data \\
\hline Christopher and Clegg, 1999121 & UK & HeartMate/Novacor & BTT & CUA & Modelled data \\
\hline Cloy et al., $1995^{122}$ & USA & HeartMate & BTT & CMA & 13 \\
\hline \multirow[t]{2}{*}{ Couper et al., $1999^{123}$} & USA & Centrifugal VAD & & & \\
\hline & & $\begin{array}{l}\text { Abiomed BVS } 5000 \\
\text { BiVAD/LVAD/RVAD }\end{array}$ & BTT/LTCS & CMA & 22 \\
\hline Gelijns et al., $1997^{124}$ & USA & $\begin{array}{l}\text { HeartMate VE/ } \\
\text { pneumatic }\end{array}$ & LTCS & Cost summation & 62 \\
\hline Loisance et al., $\left.199\right|^{125}$ & France & Not specified & BTT & $\begin{array}{l}\text { CMA, CEA: cost } \\
\text { per survivor }\end{array}$ & 37 \\
\hline Mehta et al., $1995^{126}$ & USA & Pierce-Donachy LVAD & BTT & CMA & 43 \\
\hline Morales et al., $2000^{127}$ & USA & HeartMate VE & BTT/BTR & Cost summation & 90 \\
\hline Moskowitz et al., $2000^{128}$ & USA & Not specified & BTT/LTCS & CUA & Modelled data \\
\hline Oz et al., $1997^{129}$ & USA & Not specified & LTCS & Cost & 68 \\
\hline Schiller and Reichart, $2000^{130}$ & Germany & Novacor NIO0P LVAS & BTT & Cost & 23 \\
\hline Skinner et al., $2000^{131}$ & USA & HeartMate/Thoratec & BTT & CMA & 36 \\
\hline \multicolumn{6}{|l|}{ Abstracts } \\
\hline Christensen et al., 1999132 & USA & Not specified & & CMA & 12 \\
\hline Kolbye et al., $2000^{133}$ & Denmark & HeartMate/Biomedicus & BTT & CEA: cost per LYG & Not reported \\
\hline Miller et al., $2002^{134}$ & USA & HeartMate VE & LTCS & Cost & 45 \\
\hline Mir et al., $1997^{135}$ & USA & HeartMate & BTT & CMA & 23 \\
\hline Petty and Ormanza, $1997^{136}$ & USA & HeartMate & BTT & CMA & 15 \\
\hline Schulze et al., $2000^{137}$ & Germany & Novacor & LTCS & CMA & 40 \\
\hline
\end{tabular}

TABLE 33 Summary of studies of eliciting utilities for LVAD and ESHF patients

\begin{tabular}{|c|c|c|c|c|c|}
\hline Study & Country & Device & Indication & $\begin{array}{l}\text { Derivation of } \\
\text { utilities }\end{array}$ & $\begin{array}{l}\text { Sample } \\
\text { size }\end{array}$ \\
\hline Moscowitz et al., $1997^{138}$ & USA & HeartMate & $\begin{array}{l}\text { BTT: before, during and } \\
\text { post-transplant }\end{array}$ & $\begin{array}{l}\text { Patient-based, } \\
\text { SG }\end{array}$ & 29 \\
\hline Havranek et al., $1999^{139}$ & USA & $N / A$ & $\begin{array}{l}\text { ESHF as waiting list } \\
\text { (outpatients) }\end{array}$ & $\begin{array}{l}\text { Patient-based, } \\
\text { TTO, VAS }\end{array}$ & 50 \\
\hline Lewis et al., 2001 ${ }^{140}$ & USA & $\mathrm{N} / \mathrm{A}$ & $\begin{array}{l}\text { ESHF advanced heart } \\
\text { failure, } 75 \% \text { are } \\
\text { inpatients }\end{array}$ & $\begin{array}{l}\text { Patient-based, } \\
\text { SG, TTO }\end{array}$ & 99 \\
\hline
\end{tabular}


published only as abstracts provided such limited information as to render any discussion of their methods or results of limited value. Assessment of the quality of the 13 full studies showed variability in their methodological quality and quality of reporting (see Table 34). Six studies were designated as being of 'higher quality', 120,121,124,126,127,141 with only one achieving close to a full quality rating. ${ }^{120}$ This is not unexpected given the findings of previous large-scale reviews regarding the general quality of economic studies. ${ }^{142}$

The study question was well defined in all but one of the studies. ${ }^{125}$ However, four of the 13 economic studies did not clearly state the perspective of the analysis, ${ }^{123,125-127}$ thus limiting the interpretation of the results. Three studies did not clearly state the time horizon over which costs and consequences were assessed, making interpretation of daily costs difficult. ${ }^{122,126,129,131}$ Of the 13 studies, only three had sample sizes $>50 .{ }^{124,127,129}$ Small sample sizes do not produce robust findings; they suggest that studies were not powered to detect differences in outcomes, so any findings of difference should be interpreted with caution.

Four studies did not provide a full description of the competing alternatives, making it difficult to interpret whether the findings of the studies would be applicable in a particular setting. ${ }^{122,123,129,141}$

Of the remaining eight studies that fully described the competing alternatives, four did not identify all the relevant costs and consequences associated with either the LVAD or comparator arm. ${ }^{119,121,125,131}$ Out of all 13 papers, only three studies reported quantities of resources separately from the costs. ${ }^{121,122,127}$ This means that the generalisability of the other 10 studies is limited. Nine studies reported both costs and effects, ${ }^{120,121,123,125-128,130,131}$ but only five of these studies established the effectiveness of the programmes in a transparent manner. ${ }^{120,121,126-128}$ Only four calculated an incremental costeffectiveness ratio (ICER). ${ }^{120,125,128,130}$ Of these, only one detailed the calculation to the extent that it could be replicated. ${ }^{120}$ This lack of transparency makes accurate interpretation difficult.

Misleading presentation of results was common to two studies. ${ }^{124,126}$ Mehta and colleagues ${ }^{126}$ derive a cost per day for the LVADs against comparator (a legitimate approach to costing), and conclude that LVADs, although more costly, are actually more cost-effective if considered in terms of cost per day. However, the period considered for the LVAD intervention appears to be greater than that considered for the comparator, meaning that like is not being compared with like. The presentation of cost per day is therefore misleading. In the resource trend analysis by Gelijns and colleagues, ${ }^{124}$ the reporting of probability values implies a greater significance than is strictly the case. Statistically significant does not necessarily equate with clinically significant and presentation of probability values can be misleading.

Seven of the studies did not present credible conclusions, ${ }^{119,122-126,130}$ in so far as the claims that were made in the conclusions were not fully supported by the results described in the text. Two of these studies made claims of cost-effectiveness or improved QoL where an effectiveness outcome had not been reported. ${ }^{122,123}$ In the study by Couper and colleagues, ${ }^{123}$ the LVAD intervention was deemed to be less costly over a period relative to a comparator, and so was deemed the 'costeffective' option. This is a misuse of the term. In the study by Cloy and colleagues, ${ }^{122}$ it was claimed that there was lower cost and improved QoL but QoL was not actually measured. The studies by Arabia and colleagues, ${ }^{119}$ Loisance and colleagues $^{125}$ and Schiller and Reichart ${ }^{130}$ all present conclusions that go beyond the scope of their results. The first claimed support for early intervention (intuitively reasonable but not demonstrated in the study findings), Loisance and colleagues ${ }^{125}$ claimed improved outcome with prophylaxis (without inclusion of a no prophylaxis option) and Schiller and Reichart ${ }^{130}$ concluded that long-term costs of LVADs were equivalent to costs associated with other chronic organ diseases but did not include any of these costs for comparison. The two remaining studies overstated the significance of their results in their conclusions. Gelijns and colleagues ${ }^{124}$ overstated the significance of a correlation between programme experience and intensive care unit (ICU) stay (the magnitude of the correlation is actually very small) and Mehta and colleagues ${ }^{126}$ drew on their results to promote early use of LVADs (again not wholly supported given the findings and the sample size of their study).

Six studies were available in abstract form only and as a consequence provided very limited information on their methodological quality. ${ }^{132-137}$ These abstracts are included in the quality assessment table for completeness of reporting. The results from the abstracts are reported, along with those from the full studies, in the subsequent sections of the review. Caution should be exercised when interpreting the results of the studies published as abstracts. 
The quality assessment of the utility studies (Table 35) used the checklist devised by Drummond and Jefferson. ${ }^{116}$ All three of the studies derived patient-based utilities using validated economic instruments such as the standard gamble (SG) and time trade-off (TTO). ${ }^{143}$ The studies were conducted in the USA, which raises questions over generalisability to the UK, especially given the likely different protocoldriven treatment regimens. Sample sizes were small and no information was provided describing how the sample sizes were calculated.

The wording of the questions used was also examined for offering respondents realistic alternatives that they could quantify. In the study by Moskowitz and colleagues, ${ }^{138}$ the SG asked patients told to compare (1) a hypothetical pill which would return them to full health versus (2) loss of their candidacy for transplantation or LVAD/relief of current symptoms (post-transplant group). In the study by Havrenak and colleagues, ${ }^{139}$ TTO patients traded off their current health state with a return to full health; in the visual analogue scale (VAS) they were asked to rate their current health on a $10-\mathrm{cm}$ line between 0 (death) and full health (1). In their TTO, Lewis and colleagues ${ }^{140}$ asked patients to trade off a 2-year life expectancy in their current health state versus a range of shorter durations in full health. The SG asked them to choose between their current health and a probability of return to full health.

\section{LVADs as a bridge to heart transplantation for people with ESHF}

\section{Simple cost analyses HeartMate}

Morales and colleagues ${ }^{127}$ followed a cohort of 90 consecutive patients in the USA undergoing LVAD implantation with HeartMate VE LVADs over the period from implant to transplantation. Resource use data were collected retrospectively over a 6 -year period in order to assess the impact of discharge to home during the period of LVAD support. Costs were presented in 1998-99 US\$. The study was made possible due to the relaxation of FDA regulations for LOS following implantation, and the increasing acceptance of the use of LVADs in a rehabilitation-enhancing capacity (as opposed to a last-resort therapeutic option). Out of the cohort of 90 patients, 44 patients (49\%) met the criteria for hospital discharge to home (see Table 36).
The non-discharge group included both inpatients and those patients released on a day-trip/onenight stay basis or to a rehabilitation centre/medically supervised housing. Outcomes measured in the study (across the $n=90$ population) were successful BTT, planned explantation or death. On each of these three outcomes the patients in the discharge group had improved outcomes relative to those patients in the non-discharged LVAD group. Some $96 \%$ of the discharged LVAD patients went on to receive transplants compared with $44 \%$ in the nondischarged group. The extra benefit associated with the discharge group is not surprising given the criteria for discharge, but still represents an important finding.

The additional benefit was achieved at considerably lower costs relative to the nondischarged arm. Table 37 indicates the key cost components associated with maintenance of a healthy outpatient, relative to the daily cost of an inpatient. The daily cost of an inpatient stay was of a much greater magnitude than the average monthly charge attached to a healthy discharged patient, indicating an increased cost-effectiveness of LVAD use in an appropriate subgroup of patients who are well enough to be discharged following implantation. The authors observe that the proportion of patients meeting the criteria for discharge (49\%) could be taken as a proxy for the proportion of patients eligible to benefit from home-based LVAD support as the figure was based on the activities of a cohort of consecutive patients over a 6-year period. However, the sample size is small, which may inhibit generalisability.

The cost estimates include FDA-mandated weekly visits, which are no longer a requirement. If these visits were reduced to one per month, the routine costs associated with the LVAD patients who were discharged (excluding readmissions) would fall to $\sim$ US $\$ 600$ per month. It is appropriate to consider the outpatients as being 'healthy' as $95 \%$ of outpatient time was spent at home. Cost components seemingly not accounted for are the cost attached to the training course undertaken by patients and their carers on the care and operation of the LVAD device and patient transportation costs. The completion of this training course was a stipulation for discharge and so the cost of the course should ideally be included in the cost calculations. No indirect costs or QoL estimates were reported. Inclusion of such costs is unlikely to reverse the findings of the study, but it should be remembered that this analysis considers the post-implantation period 
TABLE 34 Methodological quality and quality of reporting of the studies assessing the costs and cost-effectiveness of LVADs for people with ESHF

\begin{tabular}{|c|c|c|c|c|c|c|c|c|c|c|c|c|c|c|c|c|c|c|c|}
\hline \multirow[t]{2}{*}{ Quality criteria 116} & \multicolumn{13}{|c|}{ Full study } & \multicolumn{6}{|c|}{ Abstract } \\
\hline & 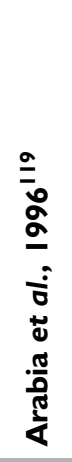 & 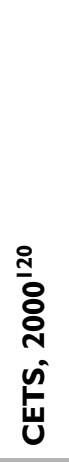 & 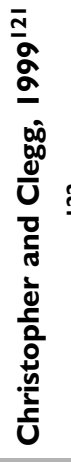 & 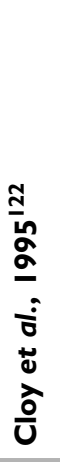 & 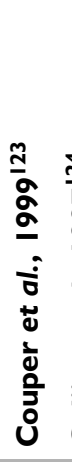 & 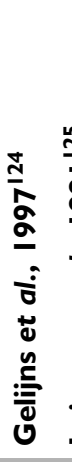 & 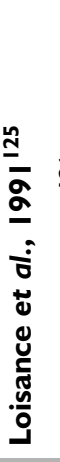 & 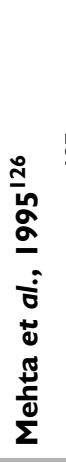 & 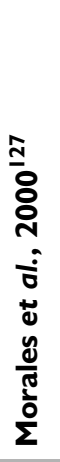 & 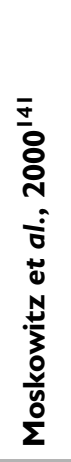 & 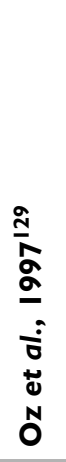 & 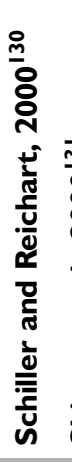 & 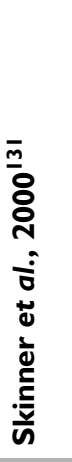 & 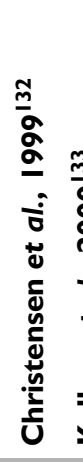 & 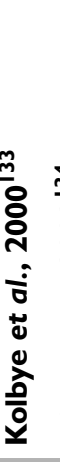 & 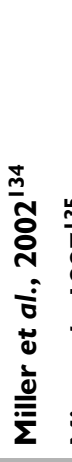 & 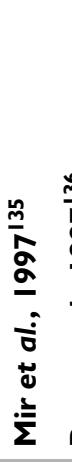 & 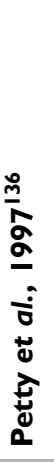 & 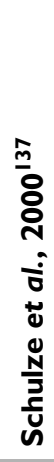 \\
\hline $\begin{array}{l}\text { Was the study question clearly } \\
\text { defined? }\end{array}$ & $\checkmark$ & $\checkmark$ & $\checkmark$ & $\checkmark$ & $\checkmark$ & $\checkmark$ & & $\checkmark$ & $\checkmark$ & $\checkmark$ & $\checkmark$ & $\checkmark$ & $\checkmark$ & & $\checkmark$ & $\checkmark$ & & & \\
\hline $\begin{array}{l}\text { What was the sample size of the } \\
\text { study? }\end{array}$ & 4 & $\mathrm{~N} / \mathrm{A}$ & & 7 & 226 & $62^{a} 3$ & 37 & 43 & 90 & $\mathrm{~N} / \mathrm{A}$ & 68 & 233 & $36^{b}$ & 12 & & 45 & 231 & 15 & 40 \\
\hline $\begin{array}{l}\text { Was the perspective of the analysis } \\
\text { clearly stated? }\end{array}$ & $\checkmark$ & $\checkmark$ & $\checkmark$ & $\checkmark$ & & $\checkmark$ & & & & $\checkmark$ & $\checkmark$ & $\checkmark$ & $\checkmark$ & & $\checkmark$ & $\checkmark$ & & & \\
\hline $\begin{array}{l}\text { Was the time horizon of costs and } \\
\text { consequences clearly stated? }\end{array}$ & $\checkmark$ & $\checkmark$ & $\checkmark$ & & $\checkmark$ & $\checkmark$ & $\checkmark$ & & $\checkmark$ & $\checkmark$ & & $\checkmark$ & & & & & & & \\
\hline $\begin{array}{l}\text { Was a comprehensive description of } \\
\text { the competing alternatives given? }\end{array}$ & $\checkmark$ & $\checkmark$ & $\checkmark$ & & & $\checkmark$ & $\checkmark$ & $\checkmark$ & $\checkmark$ & & & $\mathrm{N} / \mathrm{A}$ & $\checkmark$ & $\checkmark$ & & & & $\checkmark$ & $\checkmark$ \\
\hline $\begin{array}{l}\text { Was the effectiveness of the } \\
\text { programmes or services (appropriately) } \\
\text { established in a transparent manner? }\end{array}$ & $N / A$ & $\checkmark$ & $\checkmark 1$ & $\mathrm{~N} / \mathrm{A}$ & & $\mathrm{N} / \mathrm{A}$ & & $\checkmark$ & $\checkmark$ & $\checkmark$ & $\mathrm{N} / \mathrm{A}$ & & & $\mathrm{N} / \mathrm{A}$ & $\checkmark$ & & $\checkmark$ & & \\
\hline $\begin{array}{l}\text { Were all the relevant costs and } \\
\text { consequences for each alternative } \\
\text { identified? }\end{array}$ & & $\checkmark$ & & & & $\checkmark$ & & $\checkmark$ & $\checkmark$ & & & $\checkmark$ & & & & & & & \\
\hline $\begin{array}{l}\text { Were costs and consequences valued } \\
\text { credibly? }\end{array}$ & $\checkmark$ & $\checkmark$ & & $\checkmark$ & & $\checkmark$ & & $\checkmark$ & $\checkmark$ & $\checkmark$ & & & & & $\checkmark$ & & $\checkmark$ & $\checkmark$ & \\
\hline $\begin{array}{l}\text { Were quantities of resources reported } \\
\text { separately from costs? }\end{array}$ & & & $\checkmark$ & $\checkmark$ & & & & & $\checkmark$ & & & & & & & $\checkmark$ & & & \\
\hline $\begin{array}{l}\text { Were costs and consequences adjusted } \\
\text { for differential timing? }\end{array}$ & $N / A$ & $\checkmark$ & $\checkmark 1$ & $\mathrm{~N} / \mathrm{A}$ & & $\mathrm{N} / \mathrm{A} \mathrm{N}$ & $\mathrm{N} / \mathrm{A} \mathrm{I}$ & N/A I & $\mathrm{N} / \mathrm{A}$ & & & & & & & & & & \\
\hline $\begin{array}{l}\text { Was an incremental analysis of costs } \\
\text { and consequences of alternatives } \\
\text { performed? }\end{array}$ & $N / A$ & $\checkmark$ & & $\mathrm{N} / \mathrm{A}$ & & & $\checkmark$ & & & $\checkmark$ & & $\checkmark$ & & N/A & $\checkmark$ & & & & \\
\hline $\begin{array}{l}\text { Was the calculation of the ICER } \\
\text { transparent? }\end{array}$ & $N / A$ & $\checkmark 1$ & $\mathrm{~N} / \mathrm{A} \mathrm{I}$ & $\mathrm{N} / \mathrm{A}$ & N/A $N$ & $\mathrm{~N} / \mathrm{A}$ & & N/A I & $\mathrm{N} / \mathrm{A}$ & & $\mathrm{N} / \mathrm{A}$ & & $\mathrm{N} / \mathrm{A}$ & $\mathrm{N} / \mathrm{A}$ & & & & & \\
\hline $\begin{array}{l}\text { Was allowance made for uncertainty } \\
\text { in the estimates of costs and } \\
\text { consequences? }\end{array}$ & & & & & & & & & & & & & & & & & & & $\checkmark$ \\
\hline Sensitivity analysis & & $\checkmark$ & $\checkmark$ & & & $\checkmark$ & & & $\checkmark$ & $\checkmark$ & & & & & & & & & \\
\hline Confidence intervals & & & & & & & & $\checkmark$ & & & & & & & & & & & \\
\hline $\begin{array}{l}\text { Were credible conclusions drawn } \\
\text { from the results? }\end{array}$ & & $\checkmark$ & $\checkmark$ & & & & & & $\checkmark$ & $\checkmark$ & $\checkmark$ & $\checkmark$ & & $\checkmark$. & $\checkmark$ & $\checkmark$ & $\checkmark v$ & $\checkmark$ & \\
\hline $\begin{array}{l}\text { Ticks mean criteria satisfied; N/A, not ap } \\
{ }^{a} \text { In the Geljins study, only } 12 \text { patients we } \\
\text { resource trend data. } \\
b^{b} \text { The Skinner study assesses the impact } \\
\text { parameter but the analysis is included } \mathrm{f}\end{array}$ & of an & f & & he $\mathrm{m}$ & hylaxis & is dur & $g$ & LVAI & & than & rrt. Tl & & 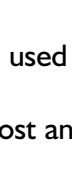 & ysis $f$ & fon & deriva & ation & & \\
\hline
\end{tabular}


TABLE 35 Methodological quality and quality of reporting of utilities studies for LVADs and ESHF

\begin{tabular}{|c|c|c|c|}
\hline & Moskowitz et al., $1997^{138}$ & Havranek et al., $1999^{139}$ & Lewis et al., $200 \mathrm{I}^{140}$ \\
\hline $\begin{array}{l}\text { Was an economic measure of } \\
\text { preference of elicitation used? }\end{array}$ & $\begin{array}{l}\checkmark \\
S G\end{array}$ & $\begin{array}{l}\checkmark \\
\text { TTO, VAS } \\
\text { (psychometric scale) }\end{array}$ & $\begin{array}{l}\checkmark \\
\text { SG, TTO }\end{array}$ \\
\hline $\begin{array}{l}\text { Subjects from whom valuations } \\
\text { were obtained }\end{array}$ & $\begin{array}{l}\checkmark \\
\text { Patients at } 3 \text { stages of LVAD } \\
\text { implantation (before } \\
\text { support, during support, } \\
\text { after heart transplantation) }\end{array}$ & $\begin{array}{l}\checkmark \\
\text { Patients in cardiology } \\
\text { clinic }\end{array}$ & $\begin{array}{l}\checkmark \\
\text { Patients in cardiology } \\
\text { hospital ward }\end{array}$ \\
\hline Sample size & 29 & 50 & 99 \\
\hline $\begin{array}{l}\text { Reporting on what subjects } \\
\text { asked to value? }\end{array}$ & $?$ & $\checkmark$ & $\checkmark$ \\
\hline Is this reasonable or appropriate? & $\begin{array}{l}\text { Lacks description of } \\
\text { consequences of candidacy } \\
\text { although patients may be } \\
\text { expected to be familiar }\end{array}$ & $\begin{array}{l}\text { Yes, patients trading-off } \\
\text { current health state }\end{array}$ & $\begin{array}{l}\text { Yes, patient value current } \\
\text { health state. Estimated } \\
\text { survival related to life } \\
\text { expectancy }\end{array}$ \\
\hline How was the sample size derived? & $\begin{array}{l}\text { No information. Poorer } \\
\text { health patients too impaired } \\
\text { for interview }\end{array}$ & $\begin{array}{l}\text { X } \\
\text { No information. Retest in } \\
\text { subsample }(n=12) \\
\text { indicated stability }\end{array}$ & $\begin{array}{l}\times \\
\text { No information }\end{array}$ \\
\hline Generalisability to UK context? & ? US population & ? US population & ? US population \\
\hline
\end{tabular}

TABLE 36 Discharge criteria ${ }^{127}$

\section{Criteria for discharging LVAD patients}

I Patient must be hospitalised for at least 30 days post-transplantation

2 Patient must currently be NYHA Class I

3 There must be echocardiographic evidence indicating that the patient's native heart has sufficient contractility to open the arterial valve and maintain an arterial pressure with the LVAD operating at its lowest rate

4 Patient must have passed the required training course in the care and operation of the device

5 Patient must be accompanied by a trained companion who has passed the required training course in the care and operation of the device

6 Patient must have in the immediate vicinity required primary and backup equipment at all times

TABLE 37 BTT costs (outpatient versus inpatient) ${ }^{127}$

\begin{tabular}{|c|c|c|c|c|}
\hline Type of cost & Unit cost (US\$) & Frequency & Weekly cost (US\$) & $\begin{array}{l}\text { Monthly cost per healthy } \\
\text { outpatient (US\$) }\end{array}$ \\
\hline Professional fees & 50 & Weekly & 50 & 200 \\
\hline Laboratory fees & 76 & Weekly & 76 & 304 \\
\hline Dressing changes & 5 & Daily & 36 & $|5|$ \\
\hline Medications & 3 & Daily & 23 & 99 \\
\hline Readmissions & 25,653 & $0.0040 /$ day & 718 & - \\
\hline Inpatient cost & 1,600 & Daily $^{a}$ & 11,200 & - \\
\hline Total & & & & 754 \\
\hline
\end{tabular}


and does not consider the acute costs associated with LVAD implantation.

\section{Novacor}

Schiller and Reichart's ${ }^{130}$ cost-effectiveness analysis calculated costs associated with the use of the Novacor LVAD as a BTT in a German healthcare setting for 23 patients with rapidly deteriorating CHF refractive to medical treatment. Costs were calculated from the perspective of both hospital and health insurer, with the overall outcome being cost (to the hospital) per day survived. Costs were presented in $1997 €$. The mean stay in the ICU was 33.1 days with a further 35.7 days of inpatient stay in a normal ward. The largest part of the expense was due to the device itself. Acute costs, excluding the costs of heart transplant, amounted to $€ 131,117$. When including the costs of heart transplant, these costs rose to $€ 175,278$ per patient. In the absence of a comparator group, it was found that for each patient there was a deficit of approximately $€ 25,500$ with regard to the amount paid out by the hospital and amount reimbursed by the health insurer. Schiller and Reichart ${ }^{130}$ calculate the average cost per day survived over a number of time periods. It is unclear which perspective these costs are calculated from (i.e. cost to hospital or cost of the deficit), but the 3-year cost per day survived is calculated at $€ 184$, the 5-year cost at $€ 122$ and the 10 -year cost at $€ 68$.

\section{Cost-minimisation analyses HeartMate}

A cost-minimisation study in the USA by Cloy and colleagues ${ }^{122}$ reported savings accrued due to outpatient release of LVAD patients relative to those patients undergoing conventional hospitalbased medical care while waiting for transplant. The year of costing was not specified in the analysis but costs are assumed to be presented in 1995 US $\$$. Cloy and colleagues ${ }^{122}$ assessed the use of the HeartMate device in a sample of six patients relative to six patients undergoing conventional medical care. Natural resource units were not reported. Cost savings were calculated based upon the difference between the duration and costs of inpatient and outpatient maintenance associated with the two treatment regimens. The savings associated with the discharged group relative to the non-discharged group were calculated at US $\$ 76,191$ per patient $(n=6)$. The authors conclude that the use of LVADs is cost saving in those patients who can be rehabilitated during the period of LVAD support. However, careful patient selection for home discharge patients in this study may have confounded results.
A further two studies from the USA were reported as abstracts. ${ }^{135,136}$ These were cost-minimisation analysis of the HeartMate LVAD as a BTT for status 1 patients at single treatment centres and were based on relatively small numbers of patients $\left(n=38\right.$, total). ${ }^{135,136}$ The year of costing was not presented but both abstracts were published in 1997. Status I patients are defined as either inpatients in the ICU receiving inotropic or mechanical support or patients who have received an LVAD. In each abstract, the HeartMate led to higher hospital charges than patients on inotropic therapy. ${ }^{135,136}$ Results were similar across the two studies: the LVAD groups had average total hospital charges of US $\$ 291,651-294,087$ whilst inotropic therapy was US $\$ 183,233-194,132 .{ }^{135,136}$

\section{Novacor}

Arabia and colleagues ${ }^{119}$ assessed the use of the Novacor LVAD in the USA in three patients in comparison to one control patient who refused the device. The year of costing is not specified but costs appear to be presented in 1995 US\$. Natural resource units were not presented. Cost savings were calculated based upon the difference between the duration and costs of inpatient and outpatient maintenance associated with the two treatment regimens. The savings associated with the discharged group relative to the non-discharged group were calculated as US $\$ 46,893$ per patient $(n=3)$. The authors conclude that the use of LVADs is cost saving in those patients who can be rehabilitated during the period of LVAD support. However, the sample size of this study is extremely limited and results should be viewed with caution.

\section{Pierce Donachy}

Mehta and colleagues ${ }^{126}$ reviewed all status I patients on the cardiac transplant waiting list (12 Pierce Donachy LVAD patients, Group 1, and 31 patients maintained on optimal medical management, Group 2) at a hospital in the USA. Costs were calculated over the period of the review, 1991-1994, and it is assumed that they are presented in 1994 US\$. Cost-minimisation analysis revealed a significant difference in the mean cost/charge associated with each group $(p<0.001)$ with Group 1 at US\$186,131/\$302,048 and Group 2 at US $\$ 100,115 / \$ 165,219$. However, when these figures were calculated on a daily basis, there was a trend towards lower costs/charges in Group $1(p>0.1)$, with Group 1 cost/charge per day calculated as US $\$ 2859 / \$ 1808$ and Group 2 as US\$3371/\$2071. There was also a trend toward increased discharge rates post-transplant in the LVAD group, but this did not reach statistical significance. 


\section{Unspecified or miscellaneous LVADs}

The study by $\mathrm{Oz}$ and colleagues, ${ }^{129}$ described as a cost-minimisation analysis in 68 patients in the USA (21 unspecified LVAD devices and 47 heart transplants), presents its methodological approach to the costing, but does not provide actual costs. Another study in the USA by Christensen and colleagues, ${ }^{132}$ presented as an abstract only, evaluates the maintenance costs for an unspecified LVAD as a BTT. The year of costing is not reported. The average cost per day of inpatient LVAD support was compared against the average costs of outpatient support, where the outpatient setting was a specially developed assisted living facility (not the patient's own home). The average cost per day for the first group $(n=5)$ was calculated as US $\$ 3441$ and for the second $(n=7)$ as US\$1357. Christensen and colleagues ${ }^{132}$ concluded that an average saving of US $\$ 2084$ per day was realised by discharging patients to the assisted-living facility with total accrued savings amounting to US $\$ 1,469,220$ across the patient group. These calculations did not include the costs of laboratory or professional fees.

Several other cost-minimisation analyses were retrieved but either focused on the effects of prophylaxis or failed to draw a distinction between the type of devices used. In the USA, Skinner and colleagues ${ }^{131}$ compared antifungal prophylaxis in LVAD patients receiving either the HeartMate or Thoratec LVADs but failed to identify the different devices in their analysis. Couper and colleagues ${ }^{123}$ undertook a study in the USA $(n=22)$ comparing an unspecified centrifugal VAD with the use of the Abiomed BVS 5000 as a BiVAD, LVAD or RVAD. No analysis was presented separately for the LVAD subgroup. Similarly, the study in France by Loisance and colleagues, ${ }^{125}$ comparing the costs and cost-effectiveness of mechanical against pharmacological BTT, grouped together TAHs, BiVADs and LVADs in the costing exercise. Further details of these studies are presented in Appendices 28 and 29.

\section{Cost-effectiveness analyses HeartMate}

A cost-effectiveness study undertaken in Denmark by Kolbye and colleagues ${ }^{133}$ reported that the HeartMate LVAD was more cost-effective than a Biomedicus (an extracorporeal blood pump) device as a BTT. The HeartMate LVAD cost a reported DKK270,000 per life-year gained (LYG) (approximately $€ 37,800$ ). This was a retrospective study that incorporated costs of devices and hospitalisation, including drug costs. The year of costing was not stated.

\section{Novacor}

The study by the Conseil d'Évaluation des Technologies de la Santé du Québec (CETS) ${ }^{120}$ in Canada examined the cost-effectiveness of the Novacor LVAD for the three indications of BTT, BTR and LTCS. ${ }^{120}$ Costs were presented in 1998 Can\$. The analysis utilised costs from a number of sources, specifically Gelijns and colleagues ${ }^{124}$ and the 'Quebec' transplant study. ${ }^{144}$ These were then applied to estimates of LYGs in the LVAD support group relative to the non-LVAD group to estimate the ICER of the LVAD intervention. The approach of the analysis is marginal in that it does not consider any of the costs which are equal across the two groups. The analysis is based on clearly defined hypothetical treatment scenarios. Costs and benefits are assessed over a 12-year period. This time frame is driven by extrapolation of 3 -year post-transplant survival rates which assumes that the average survival post-transplantation is 12 years. The costs and benefits associated with transplant following routine care are taken as the baseline, with the expected additional costs and benefits associated with 100 patients undergoing transplantation following a period of LVAD support representing the marginal costs and benefits used in determining the cost-effectiveness of the BTT scenario (see Tables 38 and 39). The authors state that it was not possible to calculate the costeffectiveness ratio in an emergency implant BTT scenario, as owing to the shortage of donor hearts no additional lives would be saved by the use of the LVAD technology (just different ones).

The ICER is calculated as Can $\$ 91,332$ per lifeyear gained $(\sim € 56,000)$ with LVAD support relative to routine care $(\mathrm{Can} \$ 117,197$ discounted at 5\%; most costs are accrued in year 1 with the benefits accrued over the 12-year period) (Table 39). A sensitivity analysis looked at the impact of assuming that a proportion (0.75) of patients would have lived for 1 year in the absence of LVAD support (thus reducing the LYGs by procedure to 185). The resultant ICER in this scenario was calculated as Can $\$ 126,304$ per lifeyear saved $(\sim 77,000)$.

\section{Cost-utility analyses \\ HeartMate}

No cost-utility studies were identified in the international literature for the BTT indication. However, Moskowitz and colleagues ${ }^{138}$ conducted a study which assessed the utilities of patients undergoing LVAD implantation as BTT. It was a comprehensive but small $(n=29)$ study conducted across all adult patients undergoing LVAD implantation at Columbia Presbyterian Medical 
TABLE 38 CETS benefits calculation ${ }^{120}$

\begin{tabular}{|lr|}
\hline Calculation component & Input \\
\hline Initial cohort $(n)$ & 143 \\
Percentage proceeding to transplant & 70 \\
Number proceeding to transplant $(n)$ & 100 \\
Increased survival $(\%)^{a}$ & 20 \\
Additional survivors $(n)$ & 20 \\
Time on LVAD support (days) & 100 \\
Survival time post-transplant (years) & 13 \\
Health benefit (life-years) & 260 \\
\hline${ }^{a}$ Based on a 20\% improvement in the 5-year survival \\
rate found by Jouveshomme and colleagues. \\
${ }^{b}$ Based on a projected estimate of $74 \%$ survival at \\
3 years.
\end{tabular}

TABLE 39 CETS cost calculation (BTT) 120

\begin{tabular}{|ll|}
\hline Cost component & Cost (Can\$) \\
\hline Cost of LVAD device & 90,000 \\
Cost of LVAD implant/heart transplant & 48,883 \\
Cost of support (per day) & 3,800 \\
Annual maintenance cost & 10,000 \\
Marginal cost LVAD & $138,883 \times 143$ \\
Marginal cost of LVAD support ${ }^{a}$ & $3,800 \times 100$ \\
Marginal cost of transplant & $48,443 \times 20$ \\
Marginal cost of maintenance & $10,000 \times 20 \times 13$ \\
Total marginal cost & $23,746,209$ \\
Cost per life-year & 91,332 \\
Cost per life-year (discounted at 5\%) & 117,197 \\
\hline a Assumed to be Can\$38/day based on Gelijns and \\
colleagues' ${ }^{\prime 24}$ estimate.
\end{tabular}

Centre over an 18-month period. The median duration of heart disease prior to implantation was 3.3 years (mean 6.5, SD 8.4, range 0.1-40.9). Patients were interviewed at three points in their treatment, prior to implantation (while in intensive care), post-implantation, during LVAD support (while hospitalised), and posttransplantation (interviews conducted in both hospital and home settings). Utilities were derived via an SG exercise, with scenarios constructed by the authors, and are summarised in Table 40. Different numbers of patients were interviewed at each point as not all patients were available for interview at each of the time periods (owing to impairment, drop-out or death). However, in order to address potential bias, the authors also conducted binary and three-way comparisons amongst those patients available for interview at the different time periods (reported below).

Moskowitz and colleagues ${ }^{138}$ calculated the reported utilities using binary comparisons (i.e. considering the responses of the same patients at two of the health states) to measure relative improvements. Significance of the difference between them was tested via paired-data analysis on 11 patients who were interviewed both before and during LVAD and 10 patients who were interviewed both during LVAD and after transplant. In each comparison, the difference between average scores varied significantly from zero even after correcting for multiple comparisons $(p=0.008,0.003)$. A further analysis was conducted based on the six patients who underwent all three interviews.

Moskowitz and colleagues ${ }^{138}$ undertook another analysis to address the issue of discarded data. In this analysis, the authors imputed data for the missing utility values during the LVAD support period and recalculated the utility associated with this period as 0.699 . The authors pointed out that this is still considerably higher than the 0.548 associated with the pre-implantation period, a figure which subsequent analyses have used as a proxy for the utility associated with non-LVAD support prior to heart transplantation. It is worth noting that the CI around the point estimate for this period is large, implying that less confidence can be attached to the accuracy of this figure.

Two other studies that examined the utilities in ESHF patients were assessed. ${ }^{139,140}$ The two studies were designed to review the suitability of the use of a utility measure in the heart-failure population. Havranek and colleagues ${ }^{139}$ conducted a study in an outpatient setting $(n=50)$ with the study population including patients awaiting cardiac transplantation (unfortunately there was no breakdown of the proportion of these patients or any further information on the NYHA classification of participants). In the study, a battery of measures were used on the patients, including TTO, SF-36 physical and mental health component scores, MLHFQ, VAS and the 6-minute walk distance. The explanation for the 0.30 difference between the TTO and VAS scores in the study by Havranek and colleagues is unclear given the transparent wording employed in each exercise (see above). In the study by Lewis and colleagues, ${ }^{140}$ participants $(n=99)$ were patients with advanced heart failure (mean LVEF 24\%), the majority (75\%) of whom were inpatients. Table 41 summarises the utility scores derived in these two studies and these appear to corroborate those reported by Moskowitz and colleagues. ${ }^{138}$

The only economic evaluation based in the UK was undertaken by Christopher and Clegg ${ }^{121}$ as 
TABLE 40 Summary of Moskowitz and colleagues' utilities ${ }^{138}$

\begin{tabular}{|c|c|c|c|}
\hline Patient condition & $\begin{array}{l}\text { Method of utility } \\
\text { derivation }\end{array}$ & Utility score (SD) & Sample size for comparisons (n) \\
\hline Before LVAD implantation & SG & $\begin{array}{l}0.548(0.276) \text { (overall score) } \\
0.566^{a}(0.236) \\
0.704^{b}(0.133)\end{array}$ & $\begin{array}{r}14 \\
11 \\
6\end{array}$ \\
\hline During LVAD support & SG & $\begin{array}{l}0.809(0.136) \text { (overall score) } \\
0.804^{a}(0.102) \\
0.826^{c}(0.149) \\
0.828^{b}(0.126)\end{array}$ & $\begin{array}{r}20 \\
11 \\
10 \\
6\end{array}$ \\
\hline Post-transplantation & SG & $\begin{array}{l}0.964(0.089) \text { (overall score) } \\
0.990^{c}(0.015) \\
0.995^{b}(0.005)\end{array}$ & $\begin{array}{r}11 \\
10 \\
6\end{array}$ \\
\hline
\end{tabular}

TABLE 4 I Summary of patient-derived ESHF mean utility scores

\begin{tabular}{|c|c|c|c|}
\hline Patient condition & $\begin{array}{l}\text { Method of utility } \\
\text { derivation }\end{array}$ & Utility score (SD) & Study description \\
\hline Waiting list (outpatients) & $\begin{array}{l}\text { TTO } \\
\text { VAS }\end{array}$ & $\begin{array}{l}0.77(\text { SD 0.28) } \\
0.47(\text { SD } 0.21)\end{array}$ & $\begin{array}{l}\text { Patient-based } \\
n=50 ; \text { Havranek et al. }{ }^{139}\end{array}$ \\
\hline Advanced heart failure ( $75 \%$ as inpatients) & $\begin{array}{l}\text { SG } \\
\text { TTO }\end{array}$ & $\begin{array}{l}0.64 \\
0.65^{b}\end{array}$ & $\begin{array}{l}\text { Patient-based } \\
n=99 ; \text { Lewis et al. }{ }^{140}\end{array}$ \\
\hline
\end{tabular}

part of a systematic review of clinical and costeffectiveness of LVADs for ESHF. The study models the cost-utility associated with the use of the HeartMate and Novacor LVADs in the UK for the BTT indication. The study presents a systematic review of the literature up to early 1999 and the cost-utility model bases its efficacy estimates on the data from the cohort study by Frazier and colleagues ${ }^{146}$ and its utility estimates on the study by Moskowitz and colleagues. ${ }^{138}$ The year of costing is not reported but it can be assumed that costs were presented in $1998 \mathrm{UK} £$. The model follows a hypothetical cohort of 100 HeartMate LVAD and 100 non-LVAD patients from pre-transplant to 20 years post-transplant. The number of patients surviving to transplant was taken from the study by Frazier and colleagues, ${ }^{146}$ where $71 \%$ of the LVAD group and $36 \%$ of the non-LVAD group survived to transplant. The duration between LVAD implantation and heart transplant is assumed to be 76 days for the LVAD group and 12 days for the non-LVAD group ${ }^{146}$ The QoL (utility) taken from the baseline estimates from the study by Moskowitz and colleagues, ${ }^{138}$ assumed to be constant at 0.548 for the non-LVAD group and 0.809 for the LVAD group. Post-transplant utility was 0.964 for both groups.

The time frame used in the analysis was 20 years with the authors assuming a linear decline in the death rate between the known values of posttransplant survival at $1(75 \%)$ and 5 years $(64 \%)$ (based on robust statistics from the National Transplant Database) and 10 years (50\%) (based on findings from one UK Transplant Centre). The quality-adjusted life-year (QALY) gain over the study period was calculated as 430 for the LVAD group and 212 for the non-LVAD group. Survival at 20 years is therefore assumed to be zero. It was assumed that both groups gain the same benefits after heart transplant. This is a conservative assumption since improved rehabilitation posttransplantation is to be expected in the LVAD arm and this may be associated with additional gain in utility in this patient group. Costs over the period were assumed to be as shown in Table 42. 
TABLE 42 Christopher and Clegg cost-effectiveness calculations ${ }^{121}$

\begin{tabular}{|c|c|c|c|}
\hline & & $\begin{array}{l}\text { LVAD group } \\
\text { (HeartMate/Novacor devices) }\end{array}$ & Non-LVAD group \\
\hline Number of patients & $a$ & 100 & 100 \\
\hline LVAD device (Trust Finance Dept) & $b$ & $£ 52,880$ & - \\
\hline LVAD procedure (Trust Finance Dept) & c & $£ 9,600$ & - \\
\hline LVAD costs (device and procedure) per patient & $d=b+c$ & $£ 62,480$ & - \\
\hline $\begin{array}{l}\text { Total LVAD procedure and device costs } \\
\text { (100 patients) }\end{array}$ & $\mathrm{e}=a \times d$ & $£ 6,247,900$ & - \\
\hline $\begin{array}{l}\text { Number of patients undergoing heart } \\
\text { transplants (i.e. surviving to transplant) }\end{array}$ & $f$ & 71 & 36 \\
\hline Heart transplant procedure (ref. costs) & $g$ & $£ 23,950$ & $£ 23,950$ \\
\hline $\begin{array}{l}\text { Heart transplant follow-up costs } \\
\text { (Trust Finance Dept) }\end{array}$ & $h$ & $£ 3,500$ & $£ 3,500$ \\
\hline $\begin{array}{l}\text { Immunosuppressant drug costs } \\
\text { (Trust protocol/BNF) }\end{array}$ & $i$ & $£ 2,890$ & $£ 2,890$ \\
\hline Total first year heart transplant first (per patient) & $j=g+h+i$ & $£ 30,340$ & $£ 30,340$ \\
\hline Total heart transplant costs & $k=j \times f$ & $£ 2,154,107$ & $£ 1,092,223$ \\
\hline $\begin{array}{l}\text { Annual follow-up heart transplant costs } \\
\text { (Trust Finance Dept) }\end{array}$ & 1 & $£ 3,500$ & \\
\hline $\begin{array}{l}\text { Annual follow-up immunosuppressant drug } \\
\text { costs (Trust protocol/BNF) }\end{array}$ & $m$ & $\notin 3,161$ & \\
\hline Total annual follow-up costs per person & $n=I+m$ & $£ 6,662$ & \\
\hline $\begin{array}{l}\text { Number of patients followed up } \\
\text { (year I onwards) }\end{array}$ & 0 & 53 & 27 \\
\hline $\begin{array}{l}\text { Total discounted heart transplant follow-up } \\
\text { costs (including drugs) } \\
\text { For calculation see report. Based on } 0 \% \\
20 \text {-year survival }\end{array}$ & $P$ & $£ 2,707,576$ & $£ \mid, 372,855$ \\
\hline $\begin{array}{l}\text { Total discounted costs (LVAD + heart } \\
\text { transplant) }\end{array}$ & $q=p+e+k$ & $£ \mid I, 109,583$ & $£ 2,465,079$ \\
\hline QALYs (discounted) & $r$ & 430 & 212 \\
\hline Incremental cost & $s$ & $£ 8,644,504$ & - \\
\hline Incremental QALYs & $t$ & 218 & - \\
\hline Incremental cost per QALY & $u=s / t$ & $£ 39,654^{a}$ & - \\
\hline
\end{tabular}

Based on 100 patients going through each arm, the discounted (costs discounted at $6 \%$, benefits at $1.5 \%$ ) the incremental cost per QALY for the LVAD group was estimated at $£ 39,790$ relative to the nonLVAD group (over a projected 20 -year period). This means that in order to gain an additional QALY in the LVAD arm relative to the non-LVAD arm, an additional $£ 39,790$ would be required. The analysis notably did not include any costs associated with the period of LVAD support (an omission for which this study has been criticised); the problems with this exclusion are discussed in more detail below. The study further attached no cost to the 12-day period of pre-transplant; as shown in the CETS analysis, ${ }^{120}$ inclusion of this figure would improve the cost-effectiveness of the LVAD arm.
The utility values chosen for use in the analysis could also be challenged. In each case the study by Christopher and Clegg ${ }^{121}$ uses the average overall (as opposed to binary or three-way comparison) utility values derived by Moskowitz and colleagues. ${ }^{138}$ These utilities were collected while patients were in the ICU and those values collected following LVAD implantation and heart transplantation may well be artificially inflated as patients have not yet had time to adjust to their new health state and are merely glad to be alive (cf. heroic intervention effect). Moskowitz ${ }^{141}$ uses an adjusted utility estimate in the calculation of cost-effectiveness: 0.75 for the period of LVAD support rather than 0.809 . This would be the conservative approach to take in this analysis, as 
although sensitivity analyses do address this issue (by calculating the ICER under minimum and maximum utility assumptions), the baseline calculation may well overestimate benefit.

\section{Summary of costs and cost- effectiveness of LVADs as BTT for patients with ESHF}

- A number of studies consider LVADs in a BTT indication, although only one study was based in the UK.

- There were no data on second-generation devices.

- The quality of these studies is mixed, although the study in the UK was of high quality.

- The studies are in different settings and conducted in different years, hence it is difficult to make credible comparisons.

- The majority of studies reviewed the use of the HeartMate device.

- No studies were found on the Jarvik, Toyobo or MicroMed DeBakey second-generation devices.

\section{HeartMate}

- One study ${ }^{127}$ found that $49 \%$ of LVAD patients were eligible for discharge during the period of support. Healthy outpatient costs were significantly lower than inpatient costs.

- One study conducted a cost-effectiveness analysis in a Danish setting ${ }^{133}$ and generated a cost per LYG of $\sim € 37,800$. It is not clear whether this includes a period of outpatient support or whether all LVAD support patients are maintained as inpatients.

- Cost-utility analysis of the use of HeartMate as BTT estimates the cost per QALY at $£ 39,790$ over a 20 -year period. ${ }^{121}$ Threshold analysis indicated that an LVAD device and procedure cost of $<19,000$ would lead to a cost per QALY of $<£ 20,000$.

\section{Novacor}

- The CETS cost-effectiveness analysis generates a cost per LYG of $€ 56,000$. This analysis assumed that patients were maintained as outpatients during the period of LVAD support.

- Schiller and Reichart ${ }^{130}$ calculate a less transparent cost-effectiveness analysis of 3 -year cost per day survived at €184 (10-year cost €68).

\section{Pierce Donachy}

- Mehta and colleagues ${ }^{126}$ conclude a trend towards lower cost/day in the LVAD group

\section{LVADs as a bridge to recovery for people with ESHF}

No costing studies or economic evaluations were found that examined the use of LVADs as a BTR for people with ESHF. The study by CETS ${ }^{120}$ highlighted the potential cost-effectiveness of LVADs as a BTR, but insufficient data were available to undertake an analysis.

\section{LVADs as a long-term chronic support for people with ESHF}

\section{Simple cost analyses HeartMate}

Gelijns and colleagues ${ }^{124}$ (also reported in Moskowitz and colleagues ${ }^{117}$ ) conducted a simple analysis of the costs associated with an LVAD programme conducted at their institution (the Columbia Presbyterian Medical Center). Costs were presented in 1995 US\$. In the main analysis, Gelijns and colleagues ${ }^{124}$ retrospectively reviewed the episodic and projected 1-year costs related to the initial hospitalisation and outpatient follow-up for 12 patients who received HeartMate VE LVADs. The authors used the ratio of cost to charges method to measure the direct costs associated with treatment (hospital and physician time). Given the FDA regulations in place at the time of the analysis (minimum LOS set to 30 days), Gelijns and colleagues calculated 'clinically sufficient' average costs alongside 'actual' average costs. ${ }^{124}$

In the analysis, the clinically sufficient costs were derived by adjusting the actual LOS to that considered clinically sufficient. Estimates of the clinically sufficient LOS were based on review of criteria such as general health status, blood results, echocardiographic evidence and evidence of appropriate management of the device on the part of patients and their companions. The average actual cost of LVAD support over a 9.5-month period (including outpatient and readmission costs) was calculated at US $\$ 221,313$; calculations based on clinically sufficient LOS reduced this estimate to US $\$ 201,148$. The focus of the study was derivation of the costs associated with the long-term use (i.e. LTCS) of the LVAD technology. A projection of the clinically sufficient 9-month cost estimated the average predicted first-year cost at US\$219,139. The breakdown of the initial hospitalisation costs for the clinically sufficient scenario is detailed in Table 43. 
TABLE 43 LVAD implantation hospitalisation costs ${ }^{\mathrm{a}}$

\begin{tabular}{|lcc|}
\hline Resource category & Average cost (SD) (US\$) & $\%$ of total cost \\
\hline LVAD (HeartMate) & 67,085 & $48 \%$ \\
Professional payment & $23,935 \pm 10,897$ & $17 \%$ \\
Special care days & $14,765 \pm 10,874$ & $10 \%$ \\
Regular floor days & $7,07 I \pm 7,376$ & $5 \%$ \\
Operating room & $10,818 \pm 1,725$ & $8 \%$ \\
Diagnostics & $3,900 \pm 3,574$ & $3 \%$ \\
Laboratory & $3,407 \pm 1,767$ & $2 \%$ \\
Blood products & $2,873 \pm 2,562$ & $2 \%$ \\
Drugs & $3,257 \pm 3,229$ & $2 \%$ \\
Miscellaneous & $3,235 \pm 1,695$ & $2 \%$ \\
Rehabilitation & $670 \pm 423$ & $0 \%$ \\
Total & $14 I, 016$ & \\
\hline a Initial hospitalisation costs only, based on 'clinically sufficient' LOS of I7.5 days. & \\
\hline
\end{tabular}

The cost of the LVAD itself accounted for nearly half of the total hospitalisation cost for implantation, with the next largest cost drivers being professional payments and special care days (comprising days in ICU, cardiac care and stepdown units). Gelijns and colleagues ${ }^{124}$ assessed the trend in resource use over time associated with implementation of the LVAD programme, with particular reference to LOS in ICU. This analysis was based on the data for 50 patients with pneumatic LVADs. On the basis of a regression analysis, the authors concluded that LOS in ICU (a significant cost driver of the LVAD programme) was inversely correlated with the time from implementation of the programme. However, this finding should be treated with some caution as, although the result was statistically significant $(p<0.05)$, the magnitude of the inverse relationship was small $\left(r^{2}=0.964\right)$.

A recent abstract from the REMATCH trial undertaking a cost-minimisation analysis, ${ }^{134}$ reported mean inpatient costs for 45 patients (66\% study population) HeartMate LVADs of US\$196,699 (median US $\$ 97,741$ ). This excluded the US\$65,000 cost of the LVAD device. ${ }^{134}$ The year of costing was not reported but the abstract was published in 2002. These inpatient costs are similar to those reported by Gelijns and colleagues. ${ }^{124}$ As the data were presented as an abstract with limited study details, caution should be exercised in their interpretation. Although the annual costs of the use of an LVAD as a BTT appear high, they compare favourably with firstyear charges (including evaluation, candidacy, organ procurement, hospitalisation, physician charges, follow-up and immunosuppression) for heart transplant patients (based on 1999 data) at US\$303,000. ${ }^{147}$

\section{Novacor}

One study published as an abstract examined the costs of the use of the Novacor LVAD as a longterm alternative to heart transplant in Germany. Costs were presented in $1999 €$. In a sample of 20 patients those receiving the Novacor LVAD had a higher mean in-hospital costs compared with those receiving a heart transplant (€62,142 vs $€ 46,874) .{ }^{137}$

\section{Cost-effectiveness analyses Novacor}

In the application of LTCS, the CETS authors calculate the cost-effectiveness of both emergency and elective implantation of the LVAD technology. ${ }^{120}$ The previous cost calculations are used, and it is again assumed that $70 \%$ of patients will survive LVAD implantation. Costs are presented in 1998 Can $\$$. A subsequent annual mortality rate of $3 \%$ is assumed (based on the International Society for Heart and Lung Transplantation Sixteenth Annual Data Report, available at http://www.ishlt.org/registry.html) average post-transplant mortality rate of $3 \%$ per year, and it is assumed that the LVAD will require replacement every 4 years with a cost equal to that of the initial implantation cost.

In the LTCS scenario, by the end of year 12, the total accumulated cost for a cohort of 100 patients is calculated at Can $\$ 38.4$ million with a gain of 641 life-years, or Can $\$ 59,842$ per LYG ( $€ 37,000)$ (Can $\$ 57,628$ discounted at 5\%). Sensitivity analysis assumes 11 days of short-term support for the non-LVAD patients at Can $\$ 5000$ per patient (the baseline calculation assumes a rapid demise at negligible cost), thus reducing the net marginal cost of the LVAD arm by Can $\$ 50$ million, resulting in a cost per LYG of Can\$52,043 ( $€ 32,000)$, 
TABLE 44 Incremental cost-effectiveness of LVADs as an LTCS ${ }^{141}$ - corrected estimates

\begin{tabular}{|c|c|c|c|}
\hline Strategy & $\begin{array}{l}\text { Incremental cost } \\
\text { (US\$) }\end{array}$ & $\begin{array}{l}\text { Incremental effectiveness } \\
\text { (QALYs) (years) }\end{array}$ & $\begin{array}{l}\text { Incremental cost-effectiveness } \\
\text { (cost per QALY) (US\$) }\end{array}$ \\
\hline \multicolumn{4}{|c|}{ Implantation LOS I 7.5 days } \\
\hline LVAD - low efficacy & 61,938 & 1.37 & 45,210 \\
\hline LVAD - high efficacy & 87,013 & 2.40 & 36,255 \\
\hline \multicolumn{4}{|c|}{ Implantation LOS 43.5 days } \\
\hline LVAD - low efficacy & 82,278 & 1.37 & 60,057 \\
\hline LVAD - high efficacy & 107,353 & 2.40 & 44,730 \\
\hline
\end{tabular}

(Can $\$ 50,075$ discounted at 5\%). The elective implantation scenario assumes that some LVAD patients would have survived in the absence of LVAD support. Assuming an average of 1-year survival, LYGs are reduced to 531 with a resultant cost-effectiveness of Can $\$ 70,903$ ( $~ € 43,000)$ (Can $\$ 67,883$ discounted at 5\%) per LYG.

The authors stress that their calculations are based on hypothetical scenarios and are not comparable with the estimates in the report by Christopher and Clegg. ${ }^{121}$ They also suggest that detailed data collection with matched controls should be made a stipulation of acceptance of the device onto Quebec's reimbursement tariff in order that more robust calculations of cost-effectiveness can be carried out. As yet there is no evidence of any such data having been published. The authors also stress that a number of decisions need to be made prior to implementation with a clear understanding that limitation of use will be essential alongside plans for additional funding.

\section{Cost-utility analyses HeartMate}

In the study by Moskowitz and colleagues, ${ }^{138}$ the authors conclude that if the utility rating associated with the period of LVAD support can be taken as a proxy for the utility associated with long-term implantation (i.e. LTCS), then such use would be acceptable on the basis of patient QoL. However, it should be noted that no long-term utility data have been collected to date and the impact of regular LVAD replacement on QoL has not been assessed. Other authors have assumed that device replacement would be necessary approximately every 4 years. ${ }^{120}$ Furthermore, the utilities reported in the study by CETS represent a point estimate derived very soon after implantation during hospitalisation. This value may therefore be an overestimate of the benefit accrued over a longer term period as patients begin to be aware of the limitations imposed by the presence of the LVAD.

In an extension of the study by Gelijns and colleagues, ${ }^{124}$ Moskowitz ${ }^{141}$ considered a hypothetical LTCS cost-effectiveness scenario, based on the findings of the original Gelijns and colleagues $^{124}$ costing paper and Moskowitz and colleagues' ${ }^{138}$ baseline utility estimates. Costs are presented in 1995 US $\$$. Moskowitz calculated the cost-utility of (only) the LVAD support period. The author highlights that there is an unusually high utility value (0.809) attached to Moskowitz and colleagues' original estimate of utility and suggests that this is likely to be an artefact of the 'thank God I'm still alive' feeling which LVAD support patients may have. The utilities derived in the study by Moskowitz were modified in order that this 'heroic intervention' effect does not confound the results, and in this instance a value of 0.75 (arbitrarily chosen and reported to approximate patients on haemodialysis) is used in the calculations of cost-utility. The study is set in the USA and, as with the original paper by Gelijns and colleagues, ${ }^{124}$ works on the premise of actual and clinically sufficient LOS driving two independent cost-effectiveness scenarios (LOS of 43.5 and 17.5 days, respectively).

In each case the cost-effectiveness ratio is presented under two assumptions of efficacy resulting in different values of cost per QALY (the details are not presented in the paper). The derivation of costs used in the exercise is unclear but it is assumed that costs reflect those calculated by Gelijns and colleagues. ${ }^{124}$ Moreover the incremental analysis (see Table 5, p. 190, in Moskowitz ${ }^{141}$ ) does not appear to use the utility data presented in the accompanying text. Table 44 recalculates the cost-effectiveness analysis using those values cited in the text. 
These results show a cost per QALY of between US $\$ 36,000$ and 60,000 , which is lower than the estimates for BTT produced by Christopher and Clegg. ${ }^{121}$ This analysis provides the only costeffectiveness data for LTCS.

\section{Summary of evidence of costs and cost-effectiveness of LVADs as an LTCS for people with ESHF}

- There are limited data available in this indication.

- The quality of the studies was mixed.

- Available data are necessarily based on hypothetical scenarios or projected short-term costs (no long-term data are as yet available for this indication).

- There were no data on second-generation LVAD devices.

\section{HeartMate}

- One study calculates the (projected) first-year cost of use of the HeartMate device at US $\$ 219,139 .{ }^{124}$

- Main cost drivers are found to be cost of the LVAD, professional payment and special care days (ICU, cardiac care and stepdown unit).

- One study calculates a cost per QALY of between US $\$ 36,000$ and 60,000 for the use of HeartMate in this indication. ${ }^{138}$

\section{Novacor}

- The CETS analysis calculates a cost per LYG of Can $\$ 57,628$ based on a hypothetical scenario. ${ }^{120}$

\section{Discussion}

Although there are a number of simple costing studies, this review has shown there is relatively little published literature on the cost-effectiveness of LVADs. A number of the costing studies reported in this review had serious methodological flaws. Even those in the 'higher quality' category had limited applicability or generalisability. The various restrictions of FDA mandated LOS for LVAD patients pre-1998 further restricts the generalisability of these studies. The major limitation of the studies in this systematic review was their limited sample size and the lack of comparators against which to judge the significance of the reported costs.

The key studies in the systematic review were the two cost summations by Gelijns and colleagues ${ }^{124}$ and Morales and colleagues 127 and the two systematic reviews by CETS ${ }^{120}$ and Christopher and Clegg. ${ }^{121}$ The two systematic reviews presented primary cost-effectiveness analyses based on modelled data. The CETS study used costs derived from Gelijns and colleagues ${ }^{124}$ and a previous study on the costs of heart transplantation. ${ }^{148}$ The study by Christopher and Clegg ${ }^{121}$ populated the model with costs based on treatment protocols, data from individual NHS trust finance departments and utilities derived in the study by Moskowitz and colleagues. ${ }^{138}$ The study by Christopher and Clegg ${ }^{121}$ provides the only example of UK-specific literature in this area.

The benefit of the model by Christopher and Clegg ${ }^{121}$ lies in its detailed consideration of survival and its use of cost-utility analysis to generate a cost per QALY figure. Rather than assuming a blanket average survival time posttransplant (as in the CETS model), the model plots the survival curve for each arm of the model. However, it should be noted that there is a difference between the estimates of survival to transplant used in the model (71 and 36\% for the LVAD and non-LVAD patients, respectively) and the assumption of marginal gain used in the model by CETs (20\% additional survival to transplant in the LVAD relative to the non-LVAD patients). ${ }^{120}$ The study by Christopher and Clegg $^{121}$ cited the figures from Frazier and colleagues, ${ }^{146}$ which includes an age range of 14-66 years in the patient group, and for that reason was excluded from the systematic review of clinical effectiveness presented in Chapter 3.

The calculations by Christopher and Clegg ${ }^{121}$ could be viewed in the light of current UK prices. The current prices for HeartMate and Novacor are $£ 48,000$ per device whereas the secondgeneration implantable MicroMed DeBakey LVAD has been priced at $£ 35,000$ per device (submission by MicroMed DeBakey). Assuming comparable efficacy, the lower of these estimates could yield savings of $£ 1.79$ million $(£ 17,880$ savings $\times 100$ patients) and the cost per QALY could fall to $£ 31,452$. Christopher and Clegg ${ }^{121}$ used threshold analysis to calculate that an LVAD device and procedure cost of $£ 19,300$ would equate to a cost per QALY of $\leq £ 20,000$.

The model developed by CETs (Novacor LVADs) was simple and transparent and provided a good template for a marginal analysis. The benefits of this model over that by Christopher and Clegg ${ }^{121}$ relate primarily to the consideration of costs associated with the period of LVAD support. The omission of these costs in the model by Christopher and Clegg ${ }^{121}$ (although pointed out 
by the authors) represents a problem that needs to be addressed. ${ }^{121}$ However, given that these costs constitute only a very small proportion of the costs associated with LVAD use, their omission, although open to criticism on methodological grounds, should not materially alter the results of any calculations.

No studies to date have attempted to generate utilities for LVADs as an LTCS, or for outpatient LVAD support. Assuming that these states have the same utility as that during LVAD BTT support may be mistaken.

The utility estimates by Moskowitz and colleagues, ${ }^{138}$ used in the model by Christopher and Clegg, ${ }^{121}$ represent the only source of patientbased utility data for LVADs patients. Bias could be introduced in terms of the small sample size (especially in the pairwise comparisons), the influence of a 'heroic intervention' effect (which may explain the CETS reluctance to use them), the fact the study was conducted between 1993 and 1995 and that the device was a firstgeneration HeartMate LVAD.

The analysis by Christopher and Clegg ${ }^{121}$ reports cost per QALY values at the boundary of acceptability given recent decision-making. ${ }^{149}$ Based on total treatment costs of both LVAD BTT support and heart transplantation, the discounted cost per QALY was $\sim £ 39,790$ (range $£ 28,510-74,000)$. However, as discussed, the assumptions underlying the costing and efficacy estimates are open to question. Moskowitz and colleagues generated acceptable threshold levels of cost-effectiveness (around US\$36,000-60,000) for the HeartMate IP LVAD as an LTCS. If this value is converted to UK currency it would appear that LVADs are more cost-effective in the LTCS indication. However, making comparisons across the two studies is problematic given the different settings, years and methodologies employed. ${ }^{138}$

Economic evaluations for the use of LVADs as an LTCS are in their infancy with good-quality trial and observational data just beginning to emerge (e.g. the REMATCH trial).

To summarise, the level of available evidence is surprisingly minimal given the serious nature of ESHF and the consistent shortfall between the number of donor organs and the number of patients requiring transplant. There is, however, a good basis for future research based on the findings and methodologies of the studies by
CETS and Christopher and Clegg. ${ }^{121}$ Further generation of utilities will allow for more robust calculations of cost per QALY.

\section{Conclusions from the systematic review of costs and cost- effectiveness of LVADs for ESHF}

- The findings from the literature review highlight a paucity of data in this area, particularly with regard to cost-utility analyses.

- Nineteen studies purporting to be economic analyses were retrieved; five studies were reported in abstract format only.

- The majority of the evidence was of poor methodological quality and ungeneralisable.

- The majority of studies described experience with the HeartMate device as a BTT, with limited consideration of other devices.

- For the BTT indication a number of studies $(n=14)$ have been published but the quality of these studies is mixed. Comparisons between studies are problematic owing to the different settings, time periods and methodologies employed in the analyses.

- Evidence from the USA suggests that outpatient management of LVAD patients is both effective and cost saving relative to inpatient management. However, controlled studies adequately powered to pick up differences in outcome are needed.

- There is limited evidence suggesting that outpatient management of LVAD patients is more cost-effective than management via optimal medical management. However, the cost of the LVAD device is not included in the derivation of these calculations.

- Only three cost-effectiveness studies have been published. These studies generally found that use of LVAD devices falls within acceptable cost boundaries, particularly when considered over the long term.

- The only cost-utility evidence for the BTT indication comes from the study by Christopher and Clegg. ${ }^{121}$ The cost per QALY of £39,790 is at the boundary of acceptability with regard to National Institute for Health and Clinical Excellence (NICE) evaluations. A reduction in device cost would lower this value; however, the analysis is also sensitive to the utility value used. There needs to be further research into the generation of utilities in ESHF patients in order to validate the utilities used in this analysis. 
- No studies have been conducted to date in the BTR indication. Results from long-term studies may show this to be an area where the use of LVADs is particularly cost-effective.

- Studies conducted in the LTCS indication $(n=4)$ are also of mixed quality.

- The limited evidence available suggests that LVADs as destination therapy are more costeffective than when used in the BTT indication. However, LTCS studies are necessarily based on modelled data and hypothetically constructed scenarios which may misrepresent actual practice and experience.

- The LTCS cost-utility analysis study calculated a cost per QALY of between US\$30,000 and US $\$ 60,000$, a value which if converted to Sterling would fall well within acceptable thresholds of cost-effectiveness. However, comparisons between the two cost-utility studies may well be misleading, as they are conducted in different settings and employ different methodologies.

- All the research to date focuses on firstgeneration devices. Consideration of secondgeneration devices is necessary in order to see if this has a significant effect on outcomes.

- The generation of utilities is of paramount importance as the only published large-scale economic evaluation uses utilities derived from a very small sample of patients. This limits the robustness of such analyses. More research in this area is essential.

- The publication of the REMATCH trial data will, it is hoped, provide much greater insight into the cost-effectiveness of LVAD devices. Data from this trial will provide a benchmark for other studies. 



\section{Chapter 5}

\section{An economic evaluation of LVADS for people with ESHF in the UK}

\section{Introduction}

The systematic review of the costs and costeffectiveness of LVADs for people with ESHF found 19 economic studies, with only one economic evaluation directly relevant to the UK. ${ }^{121}$ Although a reasonably well-conducted evaluation, the study in the UK was shown to suffer from several limitations and focused on the use of LVADs as a BTT for patients with ESHF (see Chapter 4). As a consequence, it was decided to develop a separate economic evaluation to assess the cost-effectiveness of LVADs for people with ESHF within the UK, using evidence from the systematic review of clinical effectiveness, data from published studies identified in the review of costs and cost-effectiveness and from specialist NHS hospital trusts in the UK. Although the original intention was to develop an economic evaluation for the three different indications for treatment of BTT, BTR and LTCS, insufficient data on LVADs as a BTR for people with ESHF precluded further analysis. Subsequent sections will outline the components of the economic evaluations, including the structure of the economic model, the sources of information for benefits and costs and the results of the analysis.

\section{Economic model structure}

\section{Model I: LVADs as a BTT for people with ESHF}

The economic evaluation developed for this assessment was based on a 5-year decision analytic model examining the benefits and costs of LVADs as a BTT compared with usual medical care for ESHF at the patient level. A simple deterministic approach was adopted as it involves fewer assumptions than a probabilistic approach, which, given the limited, poor-quality data available on benefits and costs, may have led to greater uncertainty. In effect, the model presents the probabilities of an average patient experiencing particular events (i.e. health states or treatment options) during the period of the evaluation, the consequences of which can be assessed in terms of benefits to the patients (survival duration and QoL) and the costs that are incurred. The model was developed using evidence from the systematic review of clinical effectiveness and costeffectiveness, and also from expert advice. It represents the pre- and post-transplant survival, QoL and costs which determine the costeffectiveness of the two alternative treatment options for people with ESHF.

The economic evaluation focused on estimating the ICER, that is, the marginal cost per QALY from using an LVAD compared with usual medical care. The intention was to allow recommendations as to the most appropriate intervention given current capacity (i.e. facilities, equipment and staff) within the NHS. As a consequence, the evaluation does not consider capital costs, training costs or other overhead costs associated with developing or providing the service. Non-direct costs were excluded from the analysis as the primary question to be addressed was the most cost-effective treatment, rather than assessing the costs of developing a service within the UK. Uncertainty in the model parameters would be investigated through probabilistic sensitivity analysis, with different values used for specific model variables to test how assumptions influence the outcome and the generalisability of the evaluation.

The model shows that patients suffering from ESHF have two treatment options, either implantation of an LVAD or the use of usual medical care. Treatment with either of these interventions may result in the person surviving to heart transplantation or, if the treatment fails, the person may die. Owing to the advanced nature of their condition, people with ESHF appear to have no other alternative treatment options. The clinical effectiveness of the different treatment options is assessed in terms of the length of patient survival gained and the QoL experienced by the patient. Importantly, the health gain resulting from either of the treatment options is determined, in part, by the availability of donor organs for heart transplantation. Although a treatment option may provide improved survival and QoL, if the availability of donor organs is limited the benefits of the technology may be modest. With declining organ donation, this scenario is an increasing possibility. 


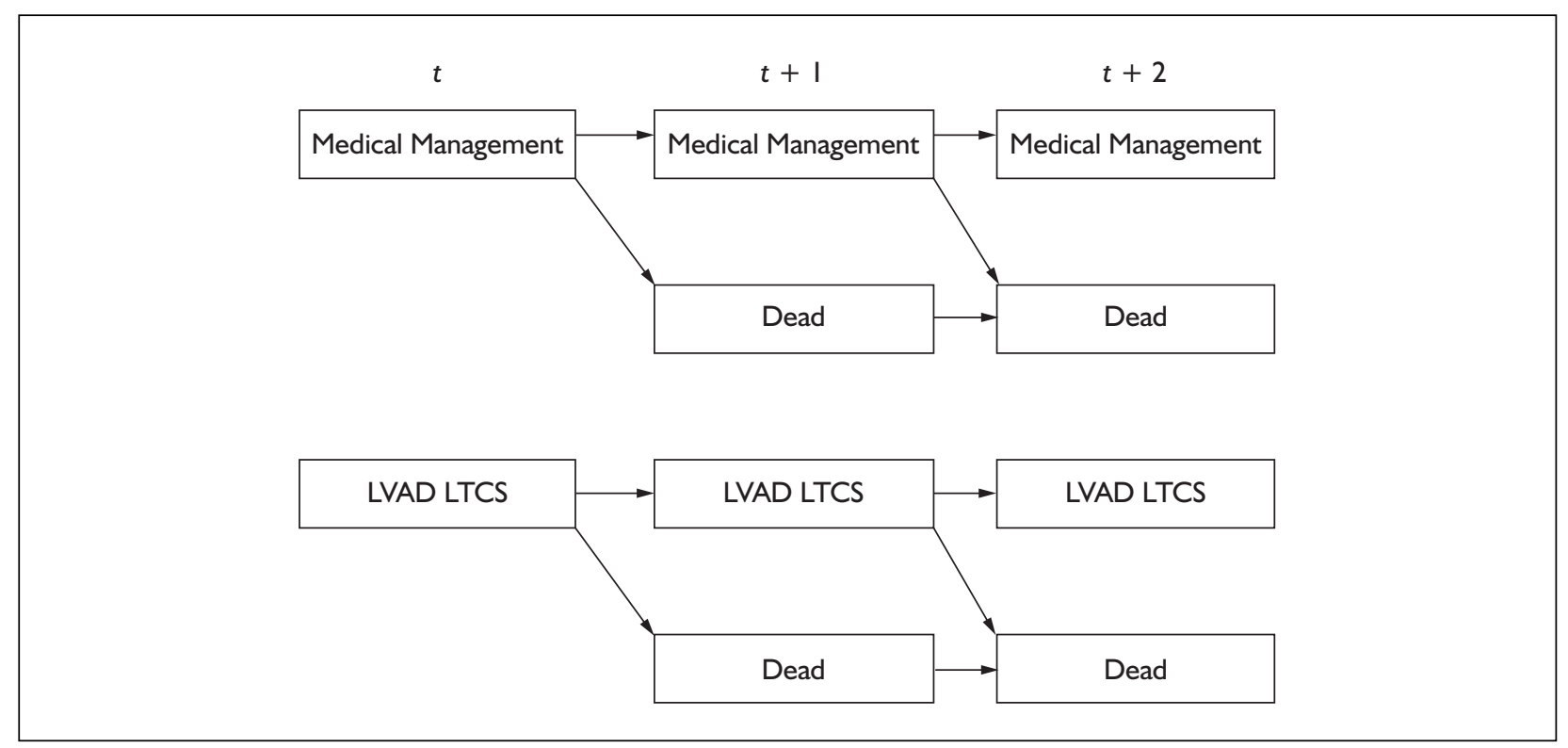

FIGURE I Pseudo-Markov model for LVAD LTCS versus medical management $(\mathrm{t}=$ time)

\section{Model 2: LVADs as LTCS for people with ESHF}

The economic evaluation for the LTCS model was based on a simple Markov methodology, which allows the modelling of uncertain processes over differing intervals. The benefits of this approach to the evaluation of health technology in cardiac care were shown by Sharples and colleagues ${ }^{150,151}$ in a retrospective analysis of a cohort of 387 heart transplant patients over a 7-year period at Papworth Hospital NHS Trust. The modelling approach has been termed a 'pseudo-Markov' approach, which, in the absence of patient-level data, uses Kaplan-Meier survival analysis data reported in a clinical trial to track patients through periods of a defined duration. The data from the survival analysis is used to populate a matrix of the probabilities of survival during the period of the evaluation.

The pseudo-Markov model developed for the assessment of LVADs as an LTCS is shown in Figure 1. As with the decision analytic model for LVADs as a BTT, the pseudo-Markov model shows that people with ESHF have the option of treatment with either usual medical care or an LVAD and that the possible outcomes are survival or death. Where the pseudo-Markov model differs from the decision analytic model is that it assesses patient survival at 3-monthly intervals over a 5 -year period reflective of the transparency of the data reported. Patient QoL through utility gains and the costs of treatment can be attached to the patients at the different periods, allowing ICERs the cost-effectiveness of the different interventions within current capacity in the NHS, excluding consideration of capital costs, training costs or other overhead costs associated with developing or providing the service. Also, the pseudo-Markov model focuses on first-generation devices, a consequence of the limited evidence available for second-generation devices such as the Jarvik 2000 and MicroMed DeBakey LVADs. The model was populated with a hypothetical cohort of 100 patients, the characteristics of which reflect evidence from the systematic review of clinical effectiveness. Sensitivity analysis was used to examine any parameter uncertainty.

The following section describes the inputs to the models, provides justification for their use, details their respective sources and explains their role in the model. As the analysis needs to reflect a NHS perspective (UK), it has tended to use UK-specific resource use and costing data where available. A number of sources were used for data including published sources, local costing data from the main centres providing an LVAD service to the NHS in the UK and expert opinion from surgeons and cardiologists within the UK.

\section{Sources of data used in the models}

\section{Efficacy - survival}

Efficacy data in terms of Kaplan-Meier survival curves for the BTT and LTCS indications were extracted from the results of the systematic review 
TABLE 45 Comparative survival by period for key BTT studies

\begin{tabular}{|c|c|c|c|c|c|c|}
\hline & \multicolumn{2}{|c|}{ Aaronson et al. ${ }^{82}$} & \multicolumn{3}{|c|}{ Massad et al. ${ }^{81}$} & \multirow{2}{*}{$\begin{array}{c}\text { Noon et al. }{ }^{91} \\
\text { LVAD }\end{array}$} \\
\hline & LVAD & $\begin{array}{l}\text { Medical } \\
\text { therapy }\end{array}$ & LVAD & $\begin{array}{l}\text { Medical } \\
\text { therapy, } \\
\text { UNOS I }\end{array}$ & $\begin{array}{l}\text { Medical } \\
\text { therapy, } \\
\text { UNOS II }\end{array}$ & \\
\hline Pre-transplant (mean, months) & 4.6 & 7.2 & $3.35^{a}$ & \multicolumn{2}{|c|}{$2.70^{a}$} & $2.19^{a}$ \\
\hline Overall survival (mean, months) & 46.26 & 29.56 & 44.61 & 44.10 & 42.32 & $\mathrm{~N} / \mathrm{R}$ \\
\hline Post-transplant survival (mean, months) & 41.66 & 22.36 & 41.26 & 41.40 & 39.62 & $N / R$ \\
\hline$\%$ reaching transplant & 80 & 74 & $\mathrm{~N} / \mathrm{R}$ & $\mathrm{N} / \mathrm{R}$ & $\mathrm{N} / \mathrm{R}$ & 52.38 \\
\hline
\end{tabular}

of clinical effectiveness (see Chapter 3). It is acknowledged that the analysis of these data may contain minor inaccuracies since they are obtained from interpreting the published survival curves, and in the absence of the raw data underlying these survival curves.

\section{Model I: LVADs as a BTT for people with ESHF}

Survival data with an indication of time to an event were limited for the assessment of LVADs as a BTT. Three studies were found that report survival data through survival curves. ${ }^{81,82,86}$ Of these, only the studies by Frazier and colleagues ${ }^{86}$ and Aaronson and colleagues ${ }^{82}$ report

Kaplan-Meier survival curves for the actual LVAD versus medical therapy 'BTT' period.

Furthermore, the study by Frazier and colleagues ${ }^{86}$ consists of a small number of patients $(n=19)$ with only two of 19 on LVAD support dying within a 2-year extended period of support. This 'extended bridge' analysis portrays a less robust survival analysis. Massad and colleagues ${ }^{81}$ report a survival curve for the post-transplant period only and failed to report the proportion of patients reaching transplant. As such, our evaluation uses the survival data from the study by Aaronson and colleagues, ${ }^{82}$ which reports survival curves for the survival to transplant, post-transplant and overall survival periods. The data comprising the survival curves was censored to reflect the number of events $\left(D_{j}\right)$ divided by the number at risk $\left(N_{j}\right)$ (i.e. after taking account of withdrawals) so that event free survival $=1-\left(D_{j} / N_{j}\right)$. It has been argued that the use of survival analysis in economic evaluation is appropriate where data have been censored. ${ }^{152}$ Hence the model has been built upon the survival data and time until transplant reported in each of these two studies with the more complete reporting in the latter forming our baseline analysis. ${ }^{81,82}$ The duration of the study by Aaronson and colleagues ${ }^{82}$ was 60 months and as a consequence no extrapolation of the results was necessary. Based on the reporting of these data, the period for the model was defined as 1 month. The area under the curve (AUC) was calculated for the overall survival period. As noted above, there may be minor inaccuracies since the raw data were not available to plot an exact curve. Aaronson and colleagues reported that the mean time to transplant was 4.6 months in the LVAD arm compared with 7.2 months in the medical therapy arm. ${ }^{82}$ The implication is that in this study the LVAD recipients were prioritised to an earlier transplant. Aaronson and colleagues highlighted that the LVAD recipients were more severely ill than their drug therapy counterparts. The AUC was 46.26 for the LVAD arm and 29.56 months for the medical arm, an increment of 16.7 months for the LVAD arm. Post-transplant survival can be calculated by subtracting pre-transplant survival from overall survival, which equates to 41.66 months in the LVAD arm compared with 22.36 months in the medical therapy arm. The proportion of patients reaching transplant was also calculated from the study by Aaronson and colleagues. ${ }^{82}$ In the LVAD arm, $73 \%$ survived to transplant and a further $9 \%$ were still awaiting transplant $(73 / 91=80 \%)$; in the medical therapy arm it was $74 \%$ (all the others died before transplantation). The data do infer some survival benefit, at least in the population studied by Aaronson and colleagues. ${ }^{82}$ These outcomes are further explored in the sensitivity analysis.

The data in the study by Massad and colleagues ${ }^{81}$ were more evenly balanced in the post-transplant period. The UNOS I status patients had a small post-transplant survival gain over the LVAD patients, whereas the UNOS II patients had a small survival decrement (Table 45). The survival curve was only reported for the post-transplant survival period with overall survival calculated by summing pre- and post-transplant survival. This 
negates the survival gain for the UNOS I patients and leads to a marginal survival benefit for the LVAD patients. Post-transplant survival was very similar to that reported by Aaronson and colleagues for the LVAD patients. Also, the shorter waiting time to transplantation for the medical therapy patients is suggestive of a more balanced prioritisation to transplantation or even some favouring of the medical patients. ${ }^{82}$ Massad and colleagues $^{81}$ show less evidence of survival benefit for LVAD patients in the BTT scenario.

Importantly, as noted above, the analyses were restricted to a first-generation HeartMate LVAD, there being insufficient data to populate a model reliably for a second-generation LVAD. However, some generalisations were made in the sensitivity analysis to simulate the possible impact of using a second-generation device. First, the cost of a second-generation device (provided by the manufacturers) was used in the analysis, while holding all other variables constant, thereby inter alia assuming efficacy equivalence. Second, the costs were combined with the efficacy data available. These consisted of data from an uncontrolled cohort study by Noon and colleagues. ${ }^{91}$ The study presented limited information on patient characteristics with no information on either mean age or UNOS/NYHA status. It reported on 32 patients receiving a MicroMed DeBakey LVAD; $81 \%$ survived to 30 days, including patients transplanted and remaining on LVAD support. Median duration of support was 47 days (mean 1.55 months, median 2.19 months). During the BTT period, 11 of the 32 patients were transplanted and 10 died, a survival to transplant percentage of $52.4 \%$ (11/21). Lacking any longer follow-up, it was assumed that survival mortality in the BTT period was equal to that of the first-generation LVADs. These data reported by Noon and colleagues ${ }^{91}$ on the MicroMed DeBakey LVAD were favoured compared with data on the Jarvik 2000 LVAD, which were based on a limited number of patients. ${ }^{95}$

\section{Model 2: LVADs as LTCS for people with ESHF}

In the LTCS scenario, only the REMATCH randomised trial assessing the HeartMate VE LVAD $^{111}$ presented a Kaplan-Meier survival curve using censored data. To extend the data reported in the REMATCH trial, survival analysis was used to calculate the impact of the LVAD and medical therapy arms over a longer duration. The geometric mean of the survival percentage was applied to give a constant risk of death in the projected period. It is evident, however, in Figure 2 that the real data (recorded up to the 10th period, 30 months) captured the majority of the survival gain.

During 20 quarterly periods, the survival gain of the LVAD LTCS arm over medical therapy was 6.84 months per person, of which 6.18 months (90\%) was within the period of the actual data and only 0.68 months captured within the projected data. Periods are described in terms of quarters reflective of the REMATCH data reporting. The baseline scenario follows the model for a duration of 20 such periods (quarters), that is, until the majority of patients are deceased. Calculations for both models were undertaken at the midpoint of each period.

\section{Comparisons between the two model populations}

Whilst the comparator for the two evaluations is medical therapy, the patient groups differ. For the BTT scenario patients will be those who are on the heart transplantation waiting list, whereas the patients in the LTCS scenario are more likely to be those whose characteristics (e.g. age) or severity of condition prevent them from consideration for heart transplantation. Although the distinction between the patient groups may reflect the clinical status of the patients, it may in part reflect the declining availability of donor organs. The REMATCH LTCS populations were exclusively NYHA Class IV with a mean age of 66-68 years (i.e. with many of the patients contraindicated for heart transplantation because of their age $>65$ years) ${ }^{153}$ whereas the BTT populations were made up of markedly younger and/or healthier patients. The mean age of patients in the study by Aaronson and colleagues ${ }^{82}$ was 49 $( \pm 13)$ years for the LVAD group and 49 $( \pm 15)$ years for the inotrope group, with $26 \%$ inotrope group classified as UNOS status 1A, 37\% status $1 \mathrm{~B}$ and $37 \%$ status 1 . Similarly, in the study by Massad and colleagues, ${ }^{81} 100 \%$ of the LVAD patients were UNOS status 1, whereas in the noLVAD group 62\% were UNOS status 1 and $38 \%$ UNOS status 2 . The mean age was 53 years in the LVAD group (range 34-66) and 50 years in the noLVAD group (range 17-66). The BTT LVAD population is therefore made up of heart transplant candidates and is a younger, healthier group of patients, far distinct from the patients who have received an LTCS LVAD and who have tended not to be candidates for heart transplant.

As stated in the research methods for the study, the economic evaluation focuses on the LVADs that were shown to be clinically effective for the different indications and relevant to the UK 


\begin{tabular}{cccccc}
\hline Intervention period (quarters) & \multicolumn{2}{c}{ Medical therapy } & & \multicolumn{2}{c}{ LVAD } \\
\cline { 2 - 3 } \cline { 5 - 6 } & Alive & Dead & & Alive & Dead \\
\hline 0 & 1.00 & 0.00 & & 1.00 & 0.00 \\
1 & 0.60 & 0.40 & & 0.75 & 0.25 \\
2 & 0.44 & 0.56 & & 0.60 & 0.40 \\
3 & 0.34 & 0.66 & & 0.58 & 0.42 \\
4 & 0.25 & 0.75 & & 0.56 & 0.44 \\
5 & 0.15 & 0.85 & & 0.47 & 0.53 \\
6 & 0.10 & 0.90 & & 0.38 & 0.62 \\
7 & 0.08 & 0.93 & 0.38 & 0.62 \\
8 & 0.04 & 0.96 & 0.22 & 0.78 \\
9 & 0.03 & 0.97 & & 0.08 & 0.92 \\
10 & 0.02 & 0.98 & 0.08 & 0.92 \\
11 & 0.01 & 0.99 & 0.06 & 0.94 \\
12 & 0.01 & 0.99 & 0.05 & 0.95 \\
13 & 0.01 & 0.99 & 0.04 & 0.96 \\
14 & 0.00 & 1.00 & 0.03 & 0.97 \\
15 & 0.00 & 1.00 & 0.02 & 0.98 \\
16 & 0.00 & 1.00 & 0.02 & 0.98 \\
17 & 0.00 & 1.00 & 0.01 & 0.99 \\
18 & 0.00 & 1.00 & 0.01 & 0.99 \\
19 & 0.00 & 1.00 & 0.01 & 0.99 \\
20 & 0.00 & 1.00 & 0.01 & 0.99 \\
21 & 0.00 & 1.00 & 0.00 & 1.00 \\
\hline
\end{tabular}

Italics, projected data.

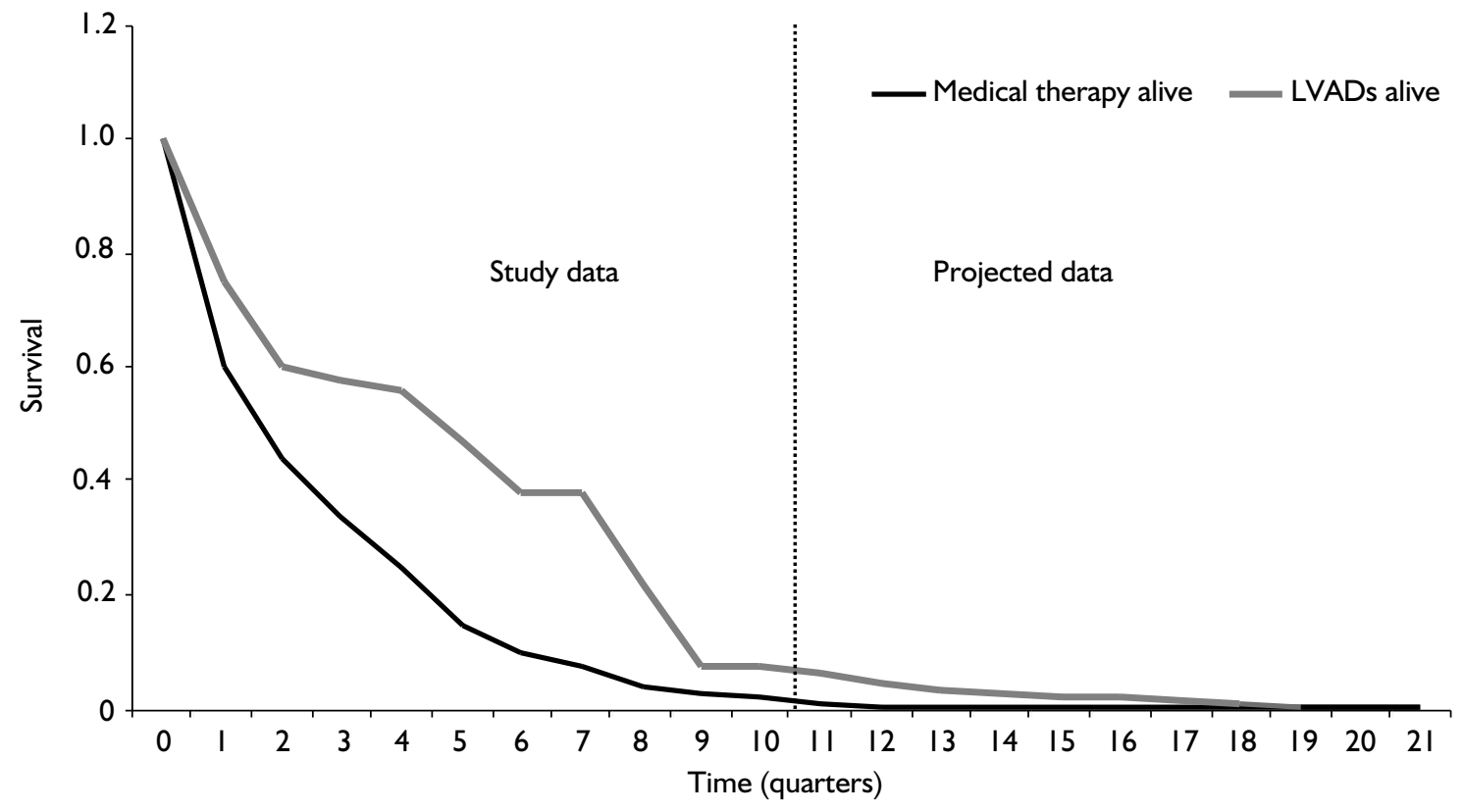

FIGURE 2 LTCS vs medical therapy - survival curves/analysis "I' (extrapolated) 
TABLE 46 Management costs following cardiac transplantation, ${ }^{150}$ inflated using HCHS indices from Netten and Curtis ${ }^{156}$

\begin{tabular}{|c|c|c|c|}
\hline & \multicolumn{3}{|c|}{ Months } \\
\hline & $\begin{array}{c}\mathrm{I}-3 \\
(\text { period I) }\end{array}$ & $\begin{array}{c}4-12 \\
\text { (periods 2-4) }\end{array}$ & $\begin{array}{c}13-60 \\
\text { (periods 5-20) }\end{array}$ \\
\hline Base costs (1995), uninflated $(£)$ & $\mathrm{I}, 188$ & 486 & 388 \\
\hline Probability of rejection & 0.11222 & 0.00931 & 0.0003 \\
\hline Probability of CMV & 0.02045 & 0.001 & 0.00001 \\
\hline Probability of infection & 0.03335 & 0.0012 & 0.00003 \\
\hline Probability of malignancy & 0 & 0.0003 & 0.00003 \\
\hline Probability of renal failure & 0 & 0 & 0.0002 \\
\hline Cost of rejection $(t)$ & 262.26 & 11.00 & 0.35 \\
\hline Cost of CMV $(t)$ & 142.54 & 7.85 & 0.09 \\
\hline Cost of infection $(t)$ & 99.75 & 1.33 & 0.04 \\
\hline Cost of malignancy $(\ell)$ & 0 & 0.28 & 0.03 \\
\hline Cost of renal failure $(t)$ & 0 & 0 & 0.34 \\
\hline Total monthly cost $(\ell)$ & 1,692 & 506 & 389 \\
\hline Inflated to 2003 prices $(E)$ & 2,105 & 630 & 484 \\
\hline
\end{tabular}

setting. As such, the evaluations of both BTT and LTCS assess the cost-effectiveness of a firstgeneration device, the HeartMate. Some tentative analyses are included for second-generation devices, the Jarvik 2000 and the MicroMed DeBakey, based on very limited data.

\section{Costs}

Cost data were obtained from a number of primary and secondary sources. These sources and the approach to calculating costs are described below for each model. Costs were divided into three headings: those associated with heart transplantation, those with medical management of patients and those associated with LVADs.

\section{Model I: LVADs as BTT for people with ESHF Heart transplantation initial and ongoing costs The mean cost of a heart transplant $(£ 14,114)$ was sourced from the NHS Reference Costs 2003. ${ }^{154}$ The reported upper and lower quartile limits (£5875-24,807) were explored in the sensitivity testing. Further, patients submitted to the heart transplantation waiting list incur assessment costs, including a period of inpatient stay, estimated at $\sim £ 3500$, which may explain why the UK waiting list is capped or 'reflective of donor supply' (Large S, Papworth Hospital NHS Trust: personal communication, 2003). One US study estimated the act of managing a patient awaiting heart transplantation is substantial at up to US\$5000 per day. ${ }^{155}$}

Following a cardiac transplantation, the management patterns in both LVAD and medical therapy arms are assumed to be equivalent. A systematic review of the management of such patients was outside the remit of this research but is well described in the study by Sharples and colleagues. ${ }^{150}$ Their audit of management patterns and associated costs at Papworth Hospital (coincidentally one of the current LVAD centres), reported management costs by period (at 1-3, 4-12, and 13-60 months) and frequency and management costs of significant adverse events (Table 46). These costs have been inflated to 2003 prices using the Hospital and Community Health Services indices from Netten and Curtis. ${ }^{156}$

The costs of the heart transplant operation are incurred for the proportions of patients in both arms surviving to transplant; the ongoing transplant management costs are incurred thereafter for the duration of survival. All patients in both arms incur the transplant assessment cost at the beginning of the model.

\section{LVAD initial and ongoing costs}

LVAD-related costs used in the baseline model were obtained from the device manufacturers and published sources. Device costs were provided by MicroMed DeBakey, one of the second-generation manufacturers. The MicroMed device was reported to cost $£ 35,000$ whereas the HeartMate and Novacor first-generation devices were $£ 48,000$ (MicroMed DeBakey submission). A submission by Jarvik Heart (personal communication, 2004) reported the price for a Jarvik 2000 heart was US $\$ 75,000$ (including US $\$ 15,000$ for external components). This was converted to UK£ prices using an exchange rate of $\$ 1.8316=£ 1 .^{157}$

LVAD costs were from one of the UK centres operating the National Specialist Commissioning 
TABLE 47 EVAD programme costs from Papworth Hospital, 2002-03

\begin{tabular}{|lcrr|}
\hline & Cost per event $(\boldsymbol{t})$ & Event rate per patient & Cost per patient $(\boldsymbol{t})$ \\
\hline Assessments $(n=21)$ & 2,202 & 1.3125 & 2,891 \\
Implant operation $(n=16)$ & 36,986 & 1 & 36,986 \\
Follow-up outpatient visit $(n=96)$ & 99 & 6 & 595 \\
Readmissions $(n=48)$ & 5,391 & 3 & 16,174 \\
Total charge excluding device & & & 56,645 \\
Average device costs & & 42,300 \\
Total charge including device & & 98,945
\end{tabular}

Advisory Group (NSCAG) national service for ventricular assist devices and their ongoing research to evaluate the service [Evaluation of the Ventricular Assist Device programme in the UK (EVAD study), HTA reference number 01/19/01]. These costs are reproduced in full in Appendix 31 and summarised in Table 47.

Importantly, these are not actual costs incurred but rather are estimates of projected costs or charges or prices to NSCAG, the funder of the EVAD study, under which the LVAD work will be undertaken. Since actual costs incurred are being recorded during the EVAD project it is likely that these will provide a more accurate representation of costs at the conclusion of the project (the UK VAD programme has thus far used a variety of devices with the most commonly used at Papworth Hospital being Thoratec and HeartMate). The implant operation and follow-up/readmission costs are significantly higher than those provided by Papworth Hospital for the study by Christopher and Clegg ${ }^{121}$ and may have been influenced by the clinical trial setting and protocols (for example, the 65-day average LOS for the implant procedure). Usefully both sets of cost data refer to the same hospital (the costs presented by Christopher and Clegg $^{121}$ have been generated bottom-up from Papworth Hospital in 1999). The assessment cost is the equivalent of the pre-heart transplant assessment cost and is very similar. The implant procedure cost is significantly higher than that presented by Christopher and Clegg. ${ }^{121}$ It is possible the real cost has increased as newer devices are more difficult to transplant or the availability of specialists has fallen, leading to higher staff costs. Interestingly, a closer examination of the breakdown of the cost shows that it is driven by ICU stay, which could again be protocol driven. However, the costs used by Christopher and Clegg ${ }^{121}$ have been criticised for being based on hospital price banding predating the NSCAG contract (Buxton M, Brunel University: personal communication, 2004). As a consequence, the baseline analysis in the economic evaluation has favoured the EVAD programme costs reported above. For upper and lower estimates the baseline costs were multiplied by a factor of $\pm 20 \%$.

All patients in the LVAD arm incur the LVAD assessment, operation and device cost. The LVAD ongoing management cost is incurred for the duration of LVAD support. The medical therapy arm does not incur any LVAD-related costs.

\section{Ongoing medical management costs}

Ongoing medical management costs for those patients not able to receive a heart transplant or LVAD were the most challenging to obtain.

Despite approaches to numerous UK bodies, including NHS trusts caring for such patients, it was not possible to obtain a reliable source. Given this, the literature and published non-UK relative resource usage have been used as a proxy vehicle to calculate a cost for medical management. This resource use data are summarised in Table 48 .

The limitations of using US data in this way are acknowledged, particularly in the light of the different treatment protocols between the two countries, but no viable alternative was available. Stewart and colleagues ${ }^{158}$ estimated that in the UK there were 988,000 individuals requiring treatment for heart failure at a cost of $£ 751$ million in 1995 . Inflating this to current prices gives $£ 876$ million at a cost per patient of $£ 887$, approximately that of LVAD management. This assumes no increase in the individuals requiring treatment, however, and will not take account of recent novel drugs or technological developments.

In this model, the period of medical management covers the cost of managing the patient through the assessment period until heart transplantation occurs. Therefore, for this model, it is the pretransplant period from Table 48 that is of most relevance. The ratio of LVAD to non-LVAD days was 5.57:1 (or 39 versus 7 days) from Aaronson and colleagues. ${ }^{82}$ This figure was applied to the 
TABLE 48 LVAD resource use data reported in clinical trials

\begin{tabular}{|c|c|c|c|c|c|}
\hline & $\begin{array}{c}\text { BTT } \\
\text { Aaronson } \\
\text { et al. }{ }^{82}\end{array}$ & $\begin{array}{c}\text { BTT } \\
\text { Massad et al. }\end{array}$ & $\begin{array}{c}\text { BTT } \\
\text { El Banayosy } \\
\text { et al. }{ }^{80 a}\end{array}$ & $\begin{array}{c}\text { BTT } \\
\text { Bank et al. }{ }^{83}\end{array}$ & $\begin{array}{c}\text { LTCS } \\
\text { Rose et al. I I }\end{array}$ \\
\hline \multicolumn{6}{|l|}{ Post-transplant LOS (days) } \\
\hline LVAD arm & 20 & 18 & $N / R$ & 23 & $N / R$ \\
\hline No-LVAD & 16 & 18 & $\mathrm{~N} / \mathrm{A}$ & 15 & $N / R$ \\
\hline Ratio LVAD: non-LVAD & 1.25 & 1.00 & $\mathrm{~N} / \mathrm{A}$ & 1.53 & N/A \\
\hline \multicolumn{6}{|l|}{ Total hospital LOS (days) } \\
\hline LVAD arm & 59 & $N / R$ & $55.6 / 58.6^{a}$ & 100 & 88 \\
\hline Non-LVAD & 23 & $N / R$ & N/A & 57 & 29 \\
\hline Ratio: I & 2.57 & $\mathrm{~N} / \mathrm{A}$ & $\mathrm{N} / \mathrm{A}$ & 1.75 & 3.03 \\
\hline \multicolumn{6}{|l|}{ Total ICU stay (days) } \\
\hline LVAD-arm & $N / R$ & $N / R$ & $16.7 / 12.2$ & 22.8 & $N / R$ \\
\hline Non-LVAD & $N / R$ & $N / R$ & $\mathrm{~N} / \mathrm{A}$ & 48.4 & $N / R$ \\
\hline Ratio: I & $\mathrm{N} / \mathrm{A}$ & $\mathrm{N} / \mathrm{A}$ & $\mathrm{N} / \mathrm{A}$ & 0.47 & $\mathrm{~N} / \mathrm{A}$ \\
\hline \multicolumn{6}{|c|}{ Pre-transplant ICU stay (days) } \\
\hline LVAD arm & $N / R$ & $N / R$ & $N / R$ & 15 & $N / R$ \\
\hline Non-LVAD & $N / R$ & $N / R$ & $N / R$ & 42 & $N / R$ \\
\hline Ratio: I & $\mathrm{N} / \mathrm{A}$ & $\mathrm{N} / \mathrm{A}$ & $\mathrm{N} / \mathrm{A}$ & 0.36 & $\mathrm{~N} / \mathrm{A}$ \\
\hline \multicolumn{6}{|l|}{ Pre-transplant LOS (days) } \\
\hline LVAD arm & 39 & $N / R$ & $N / R$ & $N / R$ & $N / R$ \\
\hline Non-LVAD & 7 & $N / R$ & $N / R$ & $N / R$ & $N / R$ \\
\hline Ratio: I & 5.57 & $\mathrm{~N} / \mathrm{A}$ & $\mathrm{N} / \mathrm{A}$ & $\mathrm{N} / \mathrm{A}$ & N/A \\
\hline
\end{tabular}

baseline analysis. For the upper bound an equivalence of cost with LVAD management was assumed, whereas for the lower bound the baseline was reduced by a factor of $20 \%$. The nearly threefold increase in ICU resource usage in the medical therapy arm reported by Bank and colleagues $^{83}$ was used.

The medical management costs are incurred in both patient groups for the duration of survival following transplant.

A summary of costs used in the BTT model is provided in Table 49.

\section{Model 2: LVADs as LTCS for people with ESHF} Heart transplantation initial and ongoing costs Heart transplantation is not included in the LTCS scenario, hence costs are not relevant. Therefore, no patients in either arm of this model incur any costs related to heart transplantation.

\section{LVAD initial and ongoing costs}

The LTCS model has used the same LVAD-based costs as detailed above for the BTT model. All assessment, operation and device cost at the start of the model. The LVAD ongoing management cost is incurred every quarter for the duration of survival in the LVAD arm. The medical therapy arm does not incur any LVAD-related costs.

\section{Ongoing medical management costs}

For the LTCS model, the period of ongoing medical management costs was likely to be longer owing to the severity of the patients' condition and nature of the indication for treatment (i.e. managing patients unsuitable for heart transplant). In Table 48, the ratio of hospitalisation resource units (the predominant cost component of managing heart failure patients) reported in the USA by Rose and colleagues $^{111}$ for LTCS was 88 days for the LVAD arm compared with 29 days for the non-LVAD arm (ratio 3.03:1). In the baseline model, this ratio was used relative to the LVAD management costs (sourced from Papworth Hospital). For the lower cost estimate the baseline was reduced by a factor of $20 \%$ whereas for upper estimate the same cost of managing LVAD patients was used.

The medical management costs are incurred every quarter in the medical therapy arm for the 
TABLE 49 Costs and sources used in the models (baseline, upper and lower estimates)

\begin{tabular}{|c|c|c|c|c|}
\hline & $\begin{array}{l}\text { Baseline } \\
((t)\end{array}$ & $\begin{array}{l}\text { Lower } \\
(\varepsilon)^{a}\end{array}$ & $\begin{array}{l}\text { Upper } \\
(E)^{a}\end{array}$ & Source \\
\hline $\begin{array}{l}\text { LVAD assessment cost } \\
\text { (both models) }\end{array}$ & 2,891 & 2,313 & 3,469 & $\begin{array}{l}\text { EVAD Project, Papworth Hospital (assessment } \\
\text { cost), } \pm 20 \%\end{array}$ \\
\hline LVAD operation (both models) & 36,986 & 29,589 & 44,383 & $\begin{array}{l}\text { EVAD Project, Papworth Hospital (implant } \\
\text { operation cost), } \pm 20 \%\end{array}$ \\
\hline \multicolumn{5}{|l|}{ LVAD device costs (both models): } \\
\hline HeartMate & 48,000 & 38,400 & 57,600 & MicroMed DeBakey submission ${ }^{159}$ \\
\hline Novacor & 48,000 & 38,400 & 57,600 & MicroMed DeBakey submission ${ }^{159}$ \\
\hline MicroMed DeBakey & 35,000 & 28,000 & 42,000 & MicroMed DeBakey submission 159 \\
\hline Jarvik & $40,948^{b}$ & 32,758 & 49,137 & $\begin{array}{l}\text { Jarvik Heart submission; includes external } \\
\text { components }{ }^{160}\end{array}$ \\
\hline $\begin{array}{l}\text { Heart transplant preparatory } \\
\text { assessment (BTT model only) }\end{array}$ & 3,500 & 2,800 & 4,200 & $\begin{array}{l}\text { S Large, Papworth Hospital NHS } \\
\text { Trust: personal communication, } 2003\end{array}$ \\
\hline $\begin{array}{l}\text { Heart transplant operation } \\
\text { (BTT model only) }\end{array}$ & 14,114 & $6,511^{c}$ & $22,970^{c}$ & NHS Reference Costs 2003, mean ${ }^{154}$ \\
\hline \multicolumn{5}{|l|}{$\begin{array}{l}\text { Ongoing management costs } \\
\text { per month (BTT model) }\end{array}$} \\
\hline LVAD & 1,397 & 1,118 & 1,677 & $\begin{array}{l}\text { EVAD Project, Papworth Hospital (cost of } \\
\text { outpatient visit and readmission), } \pm 20 \%\end{array}$ \\
\hline Medical therapy & 251 & 201 & 1,397 & $\begin{array}{l}\text { Calculated as a relative proportion of LVAD } \\
\text { resource usage (see text for details) }\end{array}$ \\
\hline \multicolumn{5}{|l|}{ Cost per quarter (LTCS model) } \\
\hline LVAD & 4,192 & 3,354 & 5,031 & $\begin{array}{l}\text { EVAD Project, Papworth Hospital (cost of } \\
\text { outpatient visit and readmission), } \pm 20 \%\end{array}$ \\
\hline Medical therapy & 1,382 & 1,105 & 4,192 & $\begin{array}{l}\text { Calculated as a relative proportion of LVAD } \\
\text { resource usage (see text for details) }\end{array}$ \\
\hline \multicolumn{5}{|c|}{$\begin{array}{l}{ }^{a} \text { Where a range of estimates was unavailable, a value of } \pm 20 \% \text { of the baseline was used for upper and lower estimates, } \\
\text { unless stated otherwise. } \\
b \text { Converted from US } \$ 75,000 \text { at } \$ 1.8316=E I \text { (Wall Street Journal Europe, } 9 \text { January 2004). }{ }^{157} \\
{ }^{c} \text { Upper and lower quartile range of unit costs. }\end{array}$} \\
\hline
\end{tabular}

duration of survival. The LVAD arm does not incur any medical management-related costs.

A summary of costs used in the LTCS model is provided in Table 49.

\section{Utilities}

There are commonly three options available to value benefits in terms of QALYs: to obtain utilities through subjective judgment (usually done by the researchers themselves, interviews with clinical experts or panels), using published utilities from the literature or adopting an approach of directly eliciting values from patients. There is an algorithm to derive utilities from the commonly used SF-36 questionnaire, but this requires the raw patient data, which investigators are often reluctant to provide. Utilities can also be generically derived (from a sample of the general population) or condition specific (from a sample of affected people). In the UK, NICE favours generically derived QALYs, which are less likely to be biased from personal interest. Utilities can also be derived directly from 'multi-attribute utility scales' (MAUS), of which the EQ-5D is one of the best-known examples. The EQ-5D consists of five multiple-choice questions covering mobility, selfcare, usual activities, pain/discomfort and anxiety/depression using a VAS. It confers only a very small burden on the patients, taking about 3 minutes to complete. Finally, there are some direct measurement techniques such as the SG and TTO methods. These require the patients to be asked a series of questions such as how much of their life they would be willing to give up in order to improve their QoL. In the TTO, patients are asked to trade off a number of years in full health to be equivalent to the rest of their life in their current (or retrospective) health state. This is performed via face-to-face interviews and requires a flipchart-type booklet for changing the values of each option until the respondent is indifferent 
TABLE 50 MLHFQ summary scores from REMATCH study and assumption underlying distribution for construction of scenarios

\begin{tabular}{|c|c|c|c|c|c|c|c|c|c|}
\hline MLHFQ subscale & Weight & $\begin{array}{l}\text { Max. } \\
\text { points }\end{array}$ & $\begin{array}{l}\text { No. of } \\
\text { items }\end{array}$ & Baseline & $\begin{array}{l}\text { Av. score } \\
\text { per item }\end{array}$ & LVAD & $\begin{array}{l}\text { Av. score } \\
\text { per item }\end{array}$ & $\begin{array}{l}\text { Medical } \\
\text { therapy }\end{array}$ & $\begin{array}{l}\text { Av. score } \\
\text { per item }\end{array}$ \\
\hline Activities of daily living & 0.19 & 20 & 4 & 14 & 3.6 & 8 & 2.0 & 11 & 2.8 \\
\hline Psychological & 0.19 & 20 & 4 & 14 & 3.6 & 8 & 2.0 & 11 & 2.8 \\
\hline Symptoms & 0.38 & 40 & 8 & 29 & 3.6 & 16 & 2.0 & 22 & 2.8 \\
\hline Socioeconomic & 0.14 & 15 & 3 & II & 3.6 & 6 & 2.0 & 8 & 2.8 \\
\hline Physical & 0.10 & 10 & 2 & 7 & 3.6 & 4 & 2.0 & 6 & 2.8 \\
\hline Total & I & 105 & & 75 & & 42 & & 58 & \\
\hline
\end{tabular}

between choices. More validity is generally attached to soliciting values from those patients living with the condition.

The availability of published evidence in this field is limited to one study ${ }^{138}$ as reported in Chapter 4.

\section{Model I: LVADs as a BTT for people with ESHF}

The model was populated using published data reported in Moskowitz and colleagues ${ }^{138}$ despite the limitations of the study identified in the systematic review. It was not possible to solicit primary patient-based utilities in the UK centres for the patients on BTT owing to the limited number of patients undergoing support and the problems associated with using historic data. Two published studies ${ }^{139,140}$ generated patient-based utilities for ESHF patients of approximately the same magnitude as the control group in the study by Moskowitz and colleagues, ${ }^{138}$ which offer some validation. In the baseline model, the strong relative estimates of the pairwise responses were used (see Table 40). The impact of other observations and variation was explored in the sensitivity analysis.

\section{Model 2: LVADs as LTCS for people with ESHF}

There were no published utilities reported for LVADs used as LTCS. Utilities have been solicited in the REMATCH study (Moskowitz AJ, Columbia University: personal communication, 2003) but have not yet been reported and were unavailable. In the baseline scenario, rather than use the published BTT utilities, utilities were solicited using a new panel-based approach developed by Stein and colleagues at the Peninsular Medical School at the Universities of Exeter and Plymouth. A pilot exercise mapped the Minnesota Living with Heart Failure Questionnaire (MLHFQ) to utilities from the summarised data reported in the pivotal REMATCH study. A brief explanation of how this exercise was conducted follows. (All data generated remain the intellectual property of
Stein and colleagues, comprising part of a grant funded by the Directorate of Health and Social Care South, Ref No. R/21/10.00/Ste.)

The MLHFQ is a 21-question QoL instrument divided into five domains specifically designed to measure the impact of heart failure on patients' QoL. The instrument is scored out of 105 with the lower score the better health. The REMATCH trial reported mean scores for the MLHFQ at baseline (score 75/105) and at 1 year for the LVAD (41/105) and medical therapy (58/105) arms. Scores by subscale and patient were unavailable, however. The mapping exercise assumed scores were achieved by a uniform set of responses across subscales and for each item within each subscale. This results in the imputed scores for each subscale and item shown in Table 50. Variations to the assumption are assessed in the sensitivity testing.

The item scores were then rounded to whole numbers in order to construct the scenarios for the utility panel. Therefore, the baseline was 4 , medical therapy 3 and the LVAD group 2. Again, variation on the rounding exercise which could be argued to overstate the ill health of the baseline/medical groups has been examined in the sensitivity analysis. The following scenarios were then constructed using the following statements to depict each possible level within items: $1=$ very little or rarely or very slightly; $2=$ a little or occasionally or slightly; 3 = moderately or often; 4 = quite a lot or very often or severely; and $5=$ very much or always or extremely often or very severely.

The scenarios used in generation of panel utilities were specified as follows (Stein K, University of Exeter: personal communication, 2003):

Scenario - baseline

- You are restricted quite a lot in everyday things you would normally do, such as walking or climbing stairs, going places from home, 
working around the house or garden, taking part in recreational activities.

- Any physical activity brings on discomfort and symptoms occur at rest.

- It is difficult or impossible for you to work because of your condition.

- You have to take medication which gives you quite a lot of side-effects and you are very often in hospital.

- You are affected quite a lot by feelings of worry or depression and a feeling that you have lost control over your life. You feel you are a burden to your family quite a lot.

- You very often find it difficult to concentrate or remember things.

- You very often feel tired and short of breath and need to sit or lie down during the day quite a lot.

- Your appetite is quite a lot worse than usual.

- Your ankles and legs are very often swollen.

- Sleeping is often difficult and you are tired and low on energy quite a lot of the time.

- It is very often difficult to have sexual activities.

\section{LVAD}

- You are restricted a little in the everyday things you would normally do, such as walking or climbing stairs, going places from home, working around the house or garden, taking part in recreational activities.

- You are comfortable at rest or with mild exertion.

- It is possible for you to work because of your condition but you have some limitations.

- You have to take medication which gives you occasional side-effects and you are occasionally in hospital.

- You are affected a little by feelings of worry or depression and a feeling that you have lost control over your life. You feel you are a burden to your family but these feelings come only a little, or occasionally.

- You find it a little difficult to concentrate or remember things.

- You feel slightly tired and short of breath and need to sit or lie down during the day occasionally.

- Your appetite is slightly worse than usual.

- Your ankles and legs are swollen and this causes slight problems.

- Sleeping is a little difficult and occasionally you are tired and low on energy.

- It is occasionally difficult to have sexual activities.

\section{Medical therapy}

- You are moderately restricted in the everyday things you would normally do, such as walking or climbing stairs, going places from home, working around the house or garden, taking part in recreational activities.

- Any physical activity brings on discomfort and symptoms occur at rest.

- It is moderately difficult for you to work because of your condition.

- You have to take medication which often gives you side-effects and you are often in hospital.

- You are moderately affected by feelings of worry or depression and a feeling that you have lost control over your life. You often feel you are a burden to your family.

- You often find it difficult to concentrate or remember things.

- You feel tired and short of breath and need to sit or lie down during the day extremely often.

- Your appetite is worse than usual.

- Your ankles and legs are often swollen.

- Sleeping is moderately difficult and you are moderately tired and low on energy.

- It is often difficult to have sexual activities.

A panel of 12 members was asked to attach utilities to these scenarios, which represented each of the baseline, LVAD, and drug therapy conditions. The results for each expert are reported in Table 51 .

Patient-based estimates would favour using the mean values, but as these are estimates of a true utility rather than a pool of patients, it was felt that the median was more appropriate. The table shows that one member gave very low scores, giving an argument in favour of using the median values. Furthermore, permitting negative utilities has also recently been shown to affect QALY estimates. ${ }^{161}$ As such, the median values from the panel have been applied in the baseline analysis. Generally, the responses were consistent, with the exception of one of the members who responded logically (i.e. rated the LVAD group $>$ the control group $>$ baseline) to the MLHFQ-generated scenarios. The effect of using the mean values is explored in the sensitivity analysis.

Importantly, the scores are similar to those patient-generated scores in the BTT study reported by Moskowitz and colleagues. ${ }^{138}$ The before implantation utility reported by Moskowitz and colleagues was 0.548 (mean of 14 patients) compared with 0.55 (median) from the utility panel, whereas the LVAD support yielded 0.809 in the study by Moskowitz and colleagues (mean of 20 patients) compared with 0.925 from the utility panel. However, it should be noted that the MLHFQ scores are measured at 1 year and it 
TABLE 5 I MLHFQ mapped utilities for LTCS and medical therapy patients

\begin{tabular}{lccccc}
\hline & \multicolumn{5}{c}{ Patient group } \\
$\begin{array}{l}\text { Utility panel } \\
\text { member }\end{array}$ & $\begin{array}{c}\text { Baseline, } \\
\text { MLHFQ = 75 }\end{array}$ & $\begin{array}{c}\text { LVAD treatment } \\
\text { group, MLHFQ = 4I }\end{array}$ & $\begin{array}{c}\text { Control group, } \\
\text { MLHFQ = 58 }\end{array}$ & $\begin{array}{c}\text { Baseline variant } \\
\text { I }\end{array}$ & $\begin{array}{c}\text { Baseline variant } \\
\mathbf{2}\end{array}$ \\
\hline A & 0.2 & 0.875 & 0.775 & 0.4 & 0.825 \\
B & -0.8 & 0.05 & -0.625 & -0.675 & 0.55 \\
C & 0.6 & 0.925 & 0.725 & 0.55 & 0.65 \\
D & 0.45 & 0.975 & 0.7 & 0.8 & 0.925 \\
E & 0.775 & 0.925 & 0.825 & 0.775 & 0.775 \\
F & 0.425 & 0.925 & 0.625 & 0.475 & 0.8 \\
G & 0.625 & 0.875 & 0.725 & 0.375 & 0.75 \\
H & 0.725 & 0.95 & 0.975 & 0.575 & 0.825 \\
I & 0.725 & 0.925 & 0.775 & 0.725 & 0.875 \\
J & N/A & 0.725 & 0.125 & 0.925 & 0.5 \\
K & 0.5 & 0.975 & 0.55 & 0.375 & 0.775 \\
L & 0.7 & 0.925 & 0.7 & 0.725 & 0.74 \\
Mean & 0.45 & 0.84 & 0.57 & 0.55 & 0.775 \\
Median & 0.55 & 0.925 & 0.7 & & \\
\hline Source: Stein K, University of Exeter: personal communication, 2003. & & \\
\hline
\end{tabular}

might be expected that patients may have overcome any difficulties in the period of early adjustment. Furthermore, neither the utility panel nor the facilitator were aware of the existence of the study by Moskowitz and colleagues when recording their utilities.

Although the approach used was a pilot study and intended as a tentative attempt to measure utilities from an LTCS LVAD group, the utilities appear to be generally representative in terms of the other evidence available to us of the relative QoL of these patients. The patient-level utilities from the REMATCH study are eagerly anticipated to provide a comparison.

It is important to remember that the BTT utilities from the study by Moskowitz and colleagues ${ }^{138}$ and the panel-based LTCS utilities extrapolated from REMATCH data reflect the experience with first-generation HeartMate devices. There have been no attempts to generate utilities for secondgeneration LVADs and there do not appear to be any plans to do so at present. Limited QoL data were available on second-generation devices to extrapolate to the analysis. Siegenthaler and colleagues $^{99}$ in a three-patient study noted a mean postoperative MLHFQ score of 30.0 at 2 months compared with 75.2 pre-LVAD. Although the baseline score was similar to the REMATCH study population and the improvement greater, it should be noted that the sample was small. In the study by Westaby and colleagues ${ }^{95}$ of four patients receiving Jarvik $2000 \mathrm{LVAD}$, patients were in a worse state than the patients in the REMATCH study (MLHFQ pre-LVAD scores 76-89) with posttransplant scores of 24-38 for the three surviving patients. These data may be suggestive of improved QoL with second-generation devices, but because of the small numbers involved they should be treated as being highly speculative.

\section{Discounting}

Following accepted UK NHS practice, in the baseline scenario of each model costs were discounted at $6 \%$ and benefits at $1.5 \%$. In the sensitivity testing a variety of other rates were employed. In the BTT model, an annual discounting approach was taken. Costs and benefits incurred in the first year were not discounted; thereafter costs were discounted on an annual basis by multiplying the undiscounted total cost or total benefit by the respective discounting multiplier for that year. In the Markov model (LTCS), costs are discounted on a quarterly basis. Once the cost and benefit per period (quarter) have been calculated for the cohort, the discounting multiplier is applied to each period. In each case, a half-cycle adjustment is made to ensure the costs are incurred at the midpoint of each period.

\section{Sensitivity analyses}

A range of one-way scenarios were examined considering variation in all the costs, utilities, discounting and efficacy. 
Sensitivity tests applied to both models

The upper and lower estimates of costs described in Table 49 were evaluated in the sensitivity testing. As a general rule for costs, when high/low estimates were unavailable the baseline estimates were increased/reduced by a factor of $\pm 20 \%$; for the device costs themselves this may, for example, mimic the reuse of an LVAD. Discounting rates were varied for both costs (3-9\%) and benefits (1.5-6\%). Threshold analysis was undertaken on the LVAD-related costs, which would yield acceptable cost per QALY of around $£ 40,000$. Further analyses in the form of spider graphs, tornado plots, stochastic simulation, histograms, cost-effectiveness acceptability curves and scatter plots were produced where appropriate.

\section{Model I: LVADs as BTT for people with ESHF}

For the heart transplantation cost, the upper and lower quartile costs were used. Heart transplant preparatory cost were varied by $\pm 20 \%$ from the baseline, and ongoing heart transplant management costs by $\pm 20 \%$ from the estimates by Sharples and colleagues. ${ }^{150}$ Data from the study by Massad and colleagues ${ }^{81}$ were included, as were the limited data on the MicroMed DeBakey VAD reported by Noon and colleagues ${ }^{91}$ (Table 45). The pairwise and three-way patient-based utilities from Moskowitz and colleagues ${ }^{138}$ were evaluated, as were the ESHF utilities from Havranek and colleagues. ${ }^{139}$ The systematic review offers some evidence of improved QoL with second-generation devices, ${ }^{95-100}$ although no utilities are presented. Thus pre-transplant utilities were varied between no gain to perfect health in favour of the LVAD group.

\section{Model 2: LVADs as LTCS for people with ESHF}

In order to test the assumptions underlying the generation of the panel-based utilities, the impact of two different combinations of item and subscale scores that lead to the same overall score were examined. It is noteworthy that in the baseline calculation an even distribution of item and domain scores was assumed to achieve the overall summary score reported in the REMATCH study. Therefore, two alternative scenarios were developed which attempted to represent the same baseline score in different ways. One of these scenarios, which over-represented the psychological subscale of the MLHFQ, was given a much higher preference value by the utility panel than the baseline scenario. This shows how assumptions about underlying item score distributions can have a dramatic effect on the preference value for the scenario. The second variant which rated poorer on recreational pastimes, burden on or relation to family, anorexia and poor concentration or memory, was rated by the panel members very similar to the original baseline scores. Albeit in a small sample, and therefore being highly speculative, it could be postulated that the psychological domains of the MLHFQ attract relatively less weight than the other domains but that social and physical domains are approximately equal in their influence on the summary preference values. It would be interesting to compare this effect in patients experiencing the conditions. The variant utilities reported in Table 51 were also examined.

Regrettably, there were no effectiveness data which could suitably be extrapolated in a secondgeneration LTCS model. To attempt to simulate the cost-effectiveness in a younger population or, perhaps, of increased efficacy from secondgeneration devices, event-free survival was inflated by a factor of 20-60\% and a threshold analysis conducted to evaluate what level of increase in survival would lead to an acceptable costeffectiveness threshold.

\section{Quality assurance}

In addition, a health economist (Adam Lloyd) not involved in the project subjected the models to internal audit. This consisted of a series of 'top down' tests conducted to confirm that the model's outputs respond predictably and consistently to changes in the input assumptions; and a 'bottom up' review of the contents of individual cells to confirm that the model's calculations were coherent and cells were correctly referenced.

\section{Results of economic analysis}

Results for each of the models are described separately below.

\section{Model I: LVADs as a BTT for ESHF}

In the baseline analysis, the cost per QALY of a HeartMate LVAD as a BTT was $£ 65,242$. Over the 5 years of the model there were 18.3 qualityadjusted months gained in the LVAD arm at an incremental cost of $£ 99,636$ per person (Table 52). This cost per QALY figure is outwith current thresholds applied to current treatment programmes.

\section{Sensitivity tests}

The full range of one-way sensitivity tests conducted for the BTT scenario are presented in Table 53. 
TABLE 52 Baseline results from the BTT model

\begin{tabular}{|c|c|c|c|}
\hline Annual calculations & LVAD group & Medical group & Increment \\
\hline \multicolumn{4}{|l|}{ Year $I$} \\
\hline Pre-transplant survival (months) & 4.6 & 7.2 & -2.6 \\
\hline Post-transplant survival (months) & 5.20 & 0.84 & 4.365 \\
\hline Transplant assessment cost & $£ 3,500$ & $£ 3,500$ & \\
\hline LVAD cost & $£ 87,877$ & $£ 0$ & $£ 87,877$ \\
\hline Heart transplant cost & $f 11,322$ & $£ 10,444$ & $£ 878$ \\
\hline Pre-transplant cost & $£ 6,428$ & $\notin 1,806$ & $£ 4,622$ \\
\hline Post-transplant cost & $£ 9,089$ & $£ 7,451$ & $\{1,638$ \\
\hline Total cost & $E 118,216$ & $£ 23,201$ & $£ 95,015$ \\
\hline Total QAL months & 8.73 & 4.75 & 3.98 \\
\hline \multicolumn{4}{|l|}{ Year 2 - undiscounted } \\
\hline Post-transplant survival (months) & 9.41 & 5.84 & 3.57 \\
\hline Post-transplant cost & $£ 6,478$ & $£ 6,858$ & $-£ 380$ \\
\hline Total QAL months & 9.07 & 5.63 & 3.44 \\
\hline \multicolumn{4}{|l|}{ Discounted } \\
\hline Post-transplant cost & 66,111 & $£ 6,470$ & $-£ 359$ \\
\hline Total QAL months & 8.93 & 5.55 & $\notin 3$ \\
\hline \multicolumn{4}{|l|}{ Year 3 - undiscounted } \\
\hline Post-transplant survival (months) & 9.24 & 5.28 & 3.96 \\
\hline Post-transplant cost & $£ 4,470$ & $£ 2,554$ & $f 1,916$ \\
\hline Total QAL months & 8.91 & 5.09 & 3.82 \\
\hline \multicolumn{4}{|l|}{ Discounted } \\
\hline Post-transplant cost & $£ 3,978$ & $£ 2,273$ & $£ 1,705$ \\
\hline Total QAL months & 8.65 & 4.94 & 3.71 \\
\hline \multicolumn{4}{|l|}{ Year 4 - undiscounted } \\
\hline Post-transplant survival (months) & 9.24 & 5.28 & 3.96 \\
\hline Post-transplant cost & $£ 4,470$ & $£ 2,554$ & $f 1,916$ \\
\hline Total QAL months & 8.91 & 5.09 & 3.82 \\
\hline \multicolumn{4}{|l|}{ Discounted } \\
\hline Post-transplant cost & $£ 3,753$ & $£ 2,144$ & $f 1,608$ \\
\hline Total QAL months & 8.52 & 4.87 & 3.65 \\
\hline \multicolumn{4}{|l|}{ Year 5 - undiscounted } \\
\hline Post-transplant survival (months) & 9.21 & 5.28 & 3.93 \\
\hline Post-transplant cost & $£ 4,456$ & $£ 2,554$ & $E I, 902$ \\
\hline Total QAL months & 8.88 & 5.09 & 3.79 \\
\hline \multicolumn{4}{|l|}{ Discounted } \\
\hline Post-transplant cost & $£ 3,529$ & $£ 2,023$ & $f 1,506$ \\
\hline Total QAL months & 8.37 & 4.80 & 3.57 \\
\hline Total cost - discounted & $£ \mid 35,587$ & $£ 36,112$ & $£ 99,475$ \\
\hline Total QAL months - discounted & 43.20 & 24.90 & 18.30 \\
\hline$£$ per QALY gained -discounted & & & $£ 65,242$ \\
\hline
\end{tabular}

The variation of these results (shown in the final two columns) determined the basis for the following more sophisticated sensitivity analyses. Results were not sensitive to changes in the cost of a heart transplant, LVAD or post-transplant management costs, discount rates or the proportion of patients reaching transplant.

These results can be visualised diagrammatically with the aid of a spider graph (Figure 3). The circles represent various cost-effectiveness thresholds. The dark circle inside these represents the baseline cost per QALY of $£ 65,242$. Finally, the dashed line represents variation from baseline resulting from changes in input parameters (the discontinuity period is a result of the huge cost per QALY when a no survival gain assumption is introduced).

The model is sensitive to the one-off costs associated with the LVAD: assessment, operation and device. In the baseline the total is $£ 87,877$ per 
TABLE 53 One-way sensitivity analyses for LVADs as a BTT for people with ESHF

\begin{tabular}{|c|c|c|c|c|c|c|c|}
\hline \multirow{2}{*}{$\begin{array}{l}\text { Parameter varied } \\
\text { Baseline }\end{array}$} & \multirow[t]{2}{*}{$\begin{array}{l}\text { Base } \\
\text { case }\end{array}$} & \multicolumn{2}{|c|}{$\begin{array}{c}\text { Range of values } \\
\text { tested }\end{array}$} & \multicolumn{2}{|c|}{$\begin{array}{c}\text { Cost per QALY (t) } \\
\text { (range) }\end{array}$} & \multicolumn{2}{|c|}{$\begin{array}{l}\text { Variation from } \\
\text { baseline }^{a}\end{array}$} \\
\hline & & & & & & & \\
\hline LVAD operation cost & $£ 36,986$ & $£ 29,589$ & $£ 44,383$ & $£ 60,391$ & $£ 70,094$ & 0.07 & 0.07 \\
\hline LVAD management cost/month & $€ \mathrm{I}, 397$ & $E I, 118$ & $£ \mid, 677$ & $£ 64,399$ & $£ 66,086$ & 0.01 & 0.01 \\
\hline Heart transplant operation cost & $E|4| 14$, & $€ 6,511$ & $£ 22,970$ & $£ 64,932$ & $£ 65,603$ & 0.01 & 0.00 \\
\hline Medical management cost/month & $£ 251$ & $€ 201$ & $€ 1,397$ & $£ 59,828$ & $€ 65,478$ & 0.08 & 0.00 \\
\hline Post-transplant management costs & & \multicolumn{2}{|c|}{ See Table 46} & $£ 64,442$ & $£ 66,042$ & 0.03 & 0.03 \\
\hline HeartMate/Novacor device cost ${ }^{b}$ & $£ 48,000$ & $£ 38,400$ & $£ 57,600$ & $£ 58,946$ & $£ 71,539$ & 0.10 & 0.10 \\
\hline MicroMed DeBakey device ${ }^{b}$ & $£ 35,000$ & $£ 28,000$ & $£ 42,000$ & $£ 52,125$ & $£ 61,307$ & 0.20 & 0.06 \\
\hline Jarvik device cost ${ }^{b}$ & $£ 40,984$ & $£ 32,758$ & $£ 49,137$ & $£ 55,246$ & $£ 65,988$ & 0.15 & 0.01 \\
\hline Costs discount rate & $6 \%$ & $3 \%$ & $9 \%$ & $\notin 64,999$ & $£ 65,517$ & 0.00 & 0.00 \\
\hline Benefits discount rate & $1.5 \%$ & $0 \%$ & $6 \%$ & $£ 63,343$ & $£ 70,925$ & 0.03 & 0.09 \\
\hline $\begin{array}{l}\text { Assuming equal pre-transplant } \\
\text { duration }\end{array}$ & & & & $£ 64,667$ & & 0.06 & \\
\hline Assuming no overall survival gain & 29.72 & 46.90 & & $£ 497,708$ & & 6.63 & \\
\hline $\begin{array}{l}\text { Assuming equal proportions reaching } \\
\text { transplant }\end{array}$ & & & & $£ 64,667$ & & 0.01 & \\
\hline $\begin{array}{l}\text { Assuming no utility benefit in } \\
\text { pre-transplant period }\end{array}$ & & & & $€ 72,710$ & & 0.11 & \\
\hline Post-transplantation utility & 0.964 & 0.75 & I & $£ 62,866$ & $£ 84,152$ & 0.04 & 0.29 \\
\hline \multicolumn{8}{|c|}{$\begin{array}{l}{ }^{a} \text { In the last two columns, cells in italics indicate a } \geq 5 \% \text { variation from baseline; cells in bold indicate } \geq 20 \% \text { variation from } \\
\text { baseline. } \\
{ }^{b} \text { Device costs represent base case costs for the separate devices; however, the baseline used in the original model was } \\
£ 48,000 \text { for the HeartMate LVAD. }\end{array}$} \\
\hline
\end{tabular}

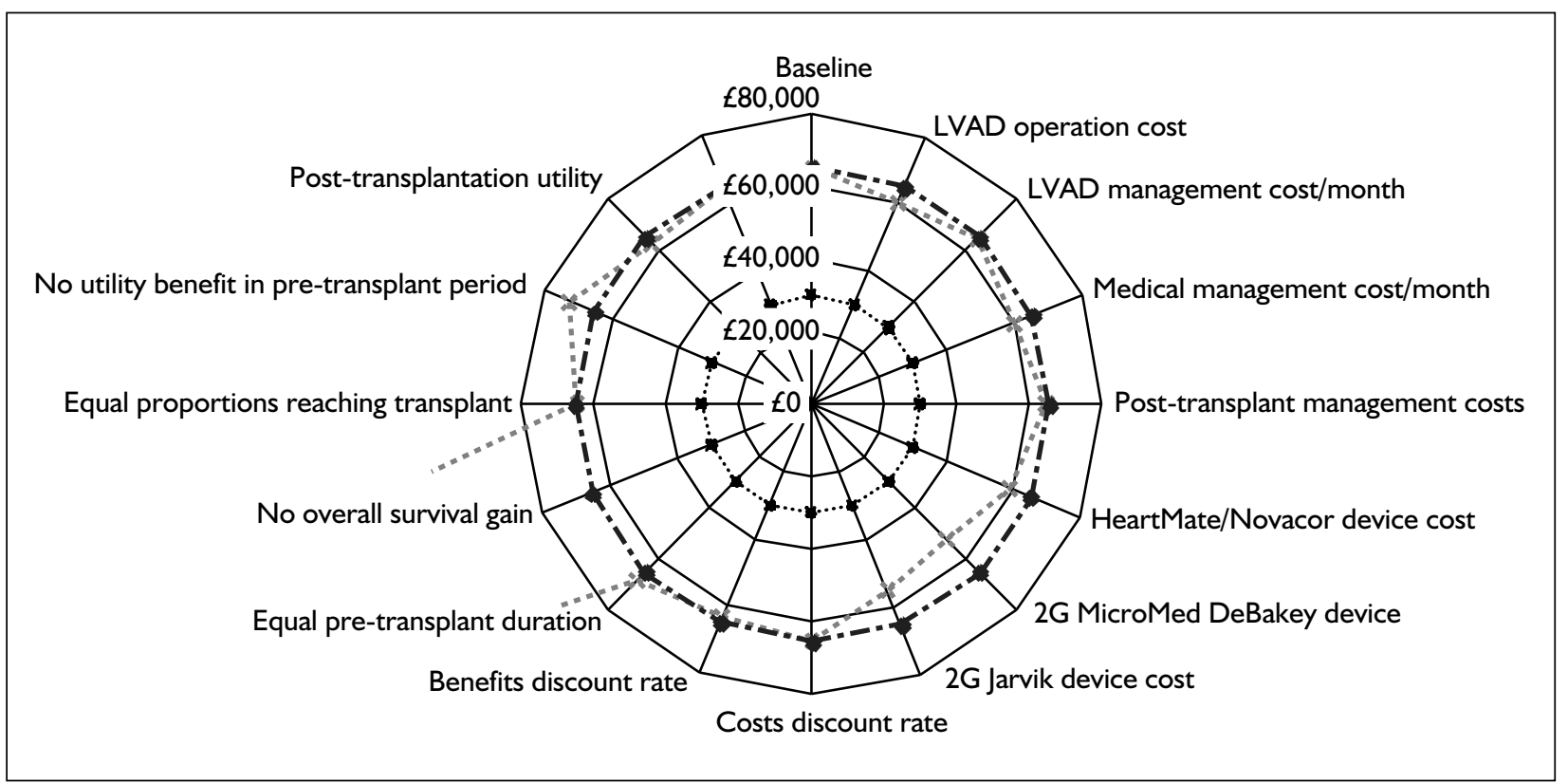

FIGURE 3 Spider graph of variation from baseline of changes in one-way parameters 


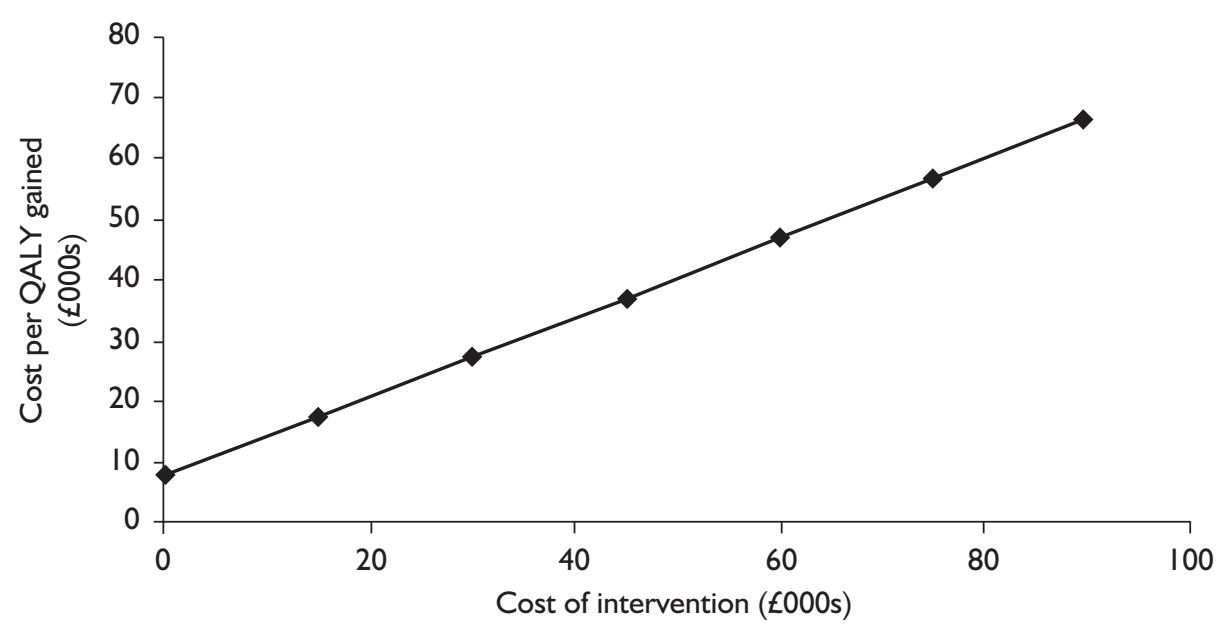

FIGURE 4 Impact of varying the initial LVAD cost (assessment, operation, device) on cost-effectiveness

patient. This is driven by the LVAD device cost $(£ 48,000)$ and operation cost $(£ 36,986)$. For the following analysis it was thought best if LVAD costs were bundled together, giving an overall package cost for an LVAD. Figure 4 shows the impact of variation in this per-person cost of the LVAD package and gives manufacturers and providers an indication of what will be the impact of the costs of their device or at their centre if these are significantly different from the estimates in our model. Only when the total cost falls below $£ 50,000$ does the intervention approach an acceptable cost-effectiveness with an incremental cost per QALY below $£ 40,000$.

Of course, these results are based on a particular study by Aaronson and colleagues, ${ }^{82}$ which showed a proven survival gain. If different inputs were used from other studies, the outcome may differ considerably. In the study by Massad and colleagues, ${ }^{81}$ the survival gain from an LVAD is eliminated and the cost per QALY unsurprisingly soars to $£ 404,289$ for the UNOS II group and $£ 1,017,630$ for the UNOS I group (Table 45). The simulated second-generation analysis using pretransplant survival and survival to transplant from Noon and colleagues, ${ }^{91}$ in conjunction with lower device costs of $£ 35,000$, produced a cost per QALY that ranged from $£ 59,642$ to $£ 366,270$. Given the limited data of Noon and colleagues, ${ }^{91}$ this analysis used the post-transplant survival reported by Aaronson and colleagues. ${ }^{82}$

To examine variability further, a stochastic simulation was conducted to determine a CI around the baseline deterministic model. Table 54 shows the parameters which comprised inputs to the stochastic modelling, together with the assumed distributions and the range of possible values. Whereas the cost and survival inputs formed a normal distribution, the utility parameters were assumed to have a beta distribution using standard deviations reported in the published studies (indicated in the column P2). The stochastic analysis randomly selects a value within each of these distributions as inputs to the model and generates the cost-effectiveness. A simulation was run 1000 times to obtain the confidence limits.

The $95 \%$ CI of the 1000 iterations was a cost per QALY of $£ 34,194$ to $£ 364,564$. A histogram (Figure 5) shows the proportion of each of the 1000 iterations to fall into each cost-effectiveness threshold. The $£ 60,000$ cost per QALY threshold contains the highest number of iterations at 164/1000.

The cost and effectiveness pairs in the stochastic analysis are used to generate a cost-effectiveness acceptability curve (CEAC). The CEAC (Figure 6) demonstrates the likelihood that the intervention is cost-effective or not cost-effective across a range of cost-effectiveness thresholds. CEACs are particularly useful when the threshold for acceptability is unknown or is likely to vary over time.

Finally, a scatter plot (Figure 7) constructed from the 1000 stochastic simulations of cost and effectiveness pairs shows that the majority of the variation in cost-effectiveness, of the variables included in the analysis, are attributable to the efficacy. Cost has relatively little impact. This 
TABLE 54 Parameters varied as inputs to the stochastic modelling

\begin{tabular}{|c|c|c|c|c|c|}
\hline & Distribution & $\mathbf{P I}$ & $\mathbf{P 2}$ & Base case & Source \\
\hline Cost of LVAD operation & Normal & $£ 27,740$ & $£ 46,233$ & $£ 36,986$ & EVAD project \\
\hline Cost of transplant operation & Normal & $£ 5,875$ & $£ 24,807$ & $E|4| \mid 4$, & $\begin{array}{l}\text { NHS Reference Costs } \\
2003^{154}\end{array}$ \\
\hline Cost per month, LVAD & Normal & $E I, 118$ & $£ \mid, 677$ & $€ 1,397$ & EVAD project \\
\hline Cost per month, medical care & Linked & $£ 176$ & $£ 326$ & $£ 251$ & $\begin{array}{l}\text { Aaronson et al. } \\
\text { (proportionate of LVAD) }\end{array}$ \\
\hline Cost per month, post-transplant & Uniform & 0.8 & 1.2 & 1.0 & $\begin{array}{l}\text { Sharples et al. }{ }^{150,151} \\
\text { (proportionate) }\end{array}$ \\
\hline Pre-transplant medical survival (months) & Normal & 7.2 & 1.49 & 7.2 & Aaronson et al. ${ }^{82}$ \\
\hline Pre-transplant LVAD survival (months) & Normal & 4.6 & 0.74 & 4.6 & Aaronson et al. ${ }^{82}$ \\
\hline Overall survival gain (\%) & Normal & 0.58 & 0.29 & 0.578 & Aaronson et al. ${ }^{82}$ \\
\hline Utility pre-transplant, medical care & Beta & 0.548 & 0.074 & 0.55 & Moskowitz et al. ${ }^{138}$ \\
\hline Utility pre-transplant, LVAD & Beta & 0.809 & 0.080 & 0.81 & Moskowitz et al. ${ }^{138}$ \\
\hline Utility, post-transplant & Beta & 0.964 & 0.027 & 0.96 & Moskowitz et al. ${ }^{138}$ \\
\hline
\end{tabular}

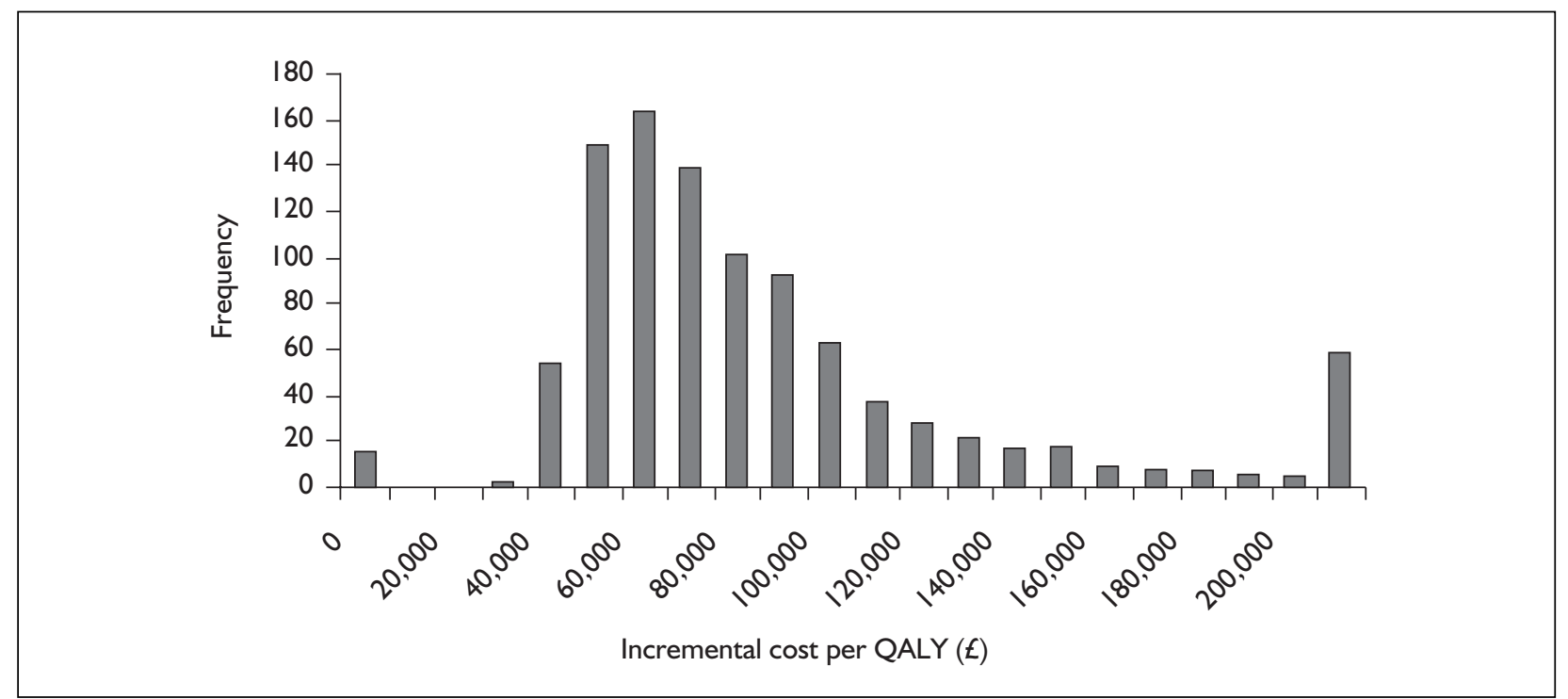

FIGURE 5 Stochastic estimate of ICER sampling distribution

analysis reinforces the need for reliable, goodquality BTT studies.

\section{Model 2: LVADs as an LTCS for ESHF}

In the baseline analysis, the incremental cost per QALY of a first-generation HeartMate LVAD as an LTCS was $£ 170,616$. This is based on HeartMatespecific data at a cost of $£ 48,000$ per device and the EVAD costs. Sixty QALYs are gained in the 100 patient cohort (0.6 QALYs per person) at an additional cost of $£ 102,000$ per patient over the 5 years, or $£ 10.2$ million for the 100 patient cohort (Table 55).

\section{Sensitivity tests}

The results of the sensitivity analysis are summarised in Table 56. The cost-effectiveness of LVADs as LTCS was not sensitive to changes in the discount rate, costs or changes in the utility assumptions within the upper and lower bounds. In all of these results the cost per QALY was well above generally accepted norms. The most uncertain variable (owing to a lack of reported data) in the analysis was the ongoing cost of medical management. In the event, even when the medical management costs are assumed equal to LVAD ongoing management costs, there is little 


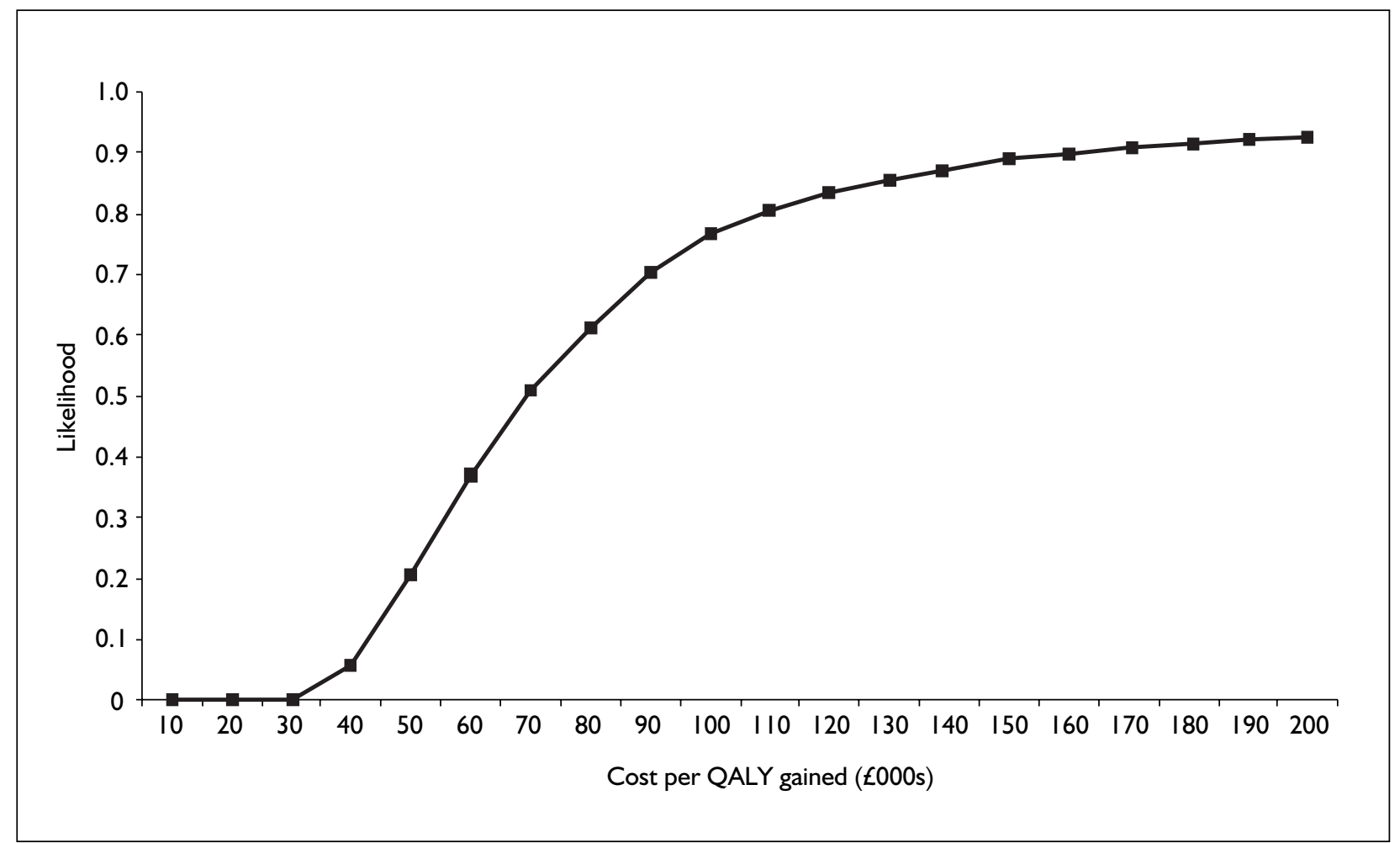

FIGURE 6 Stochastic estimate of CEAC

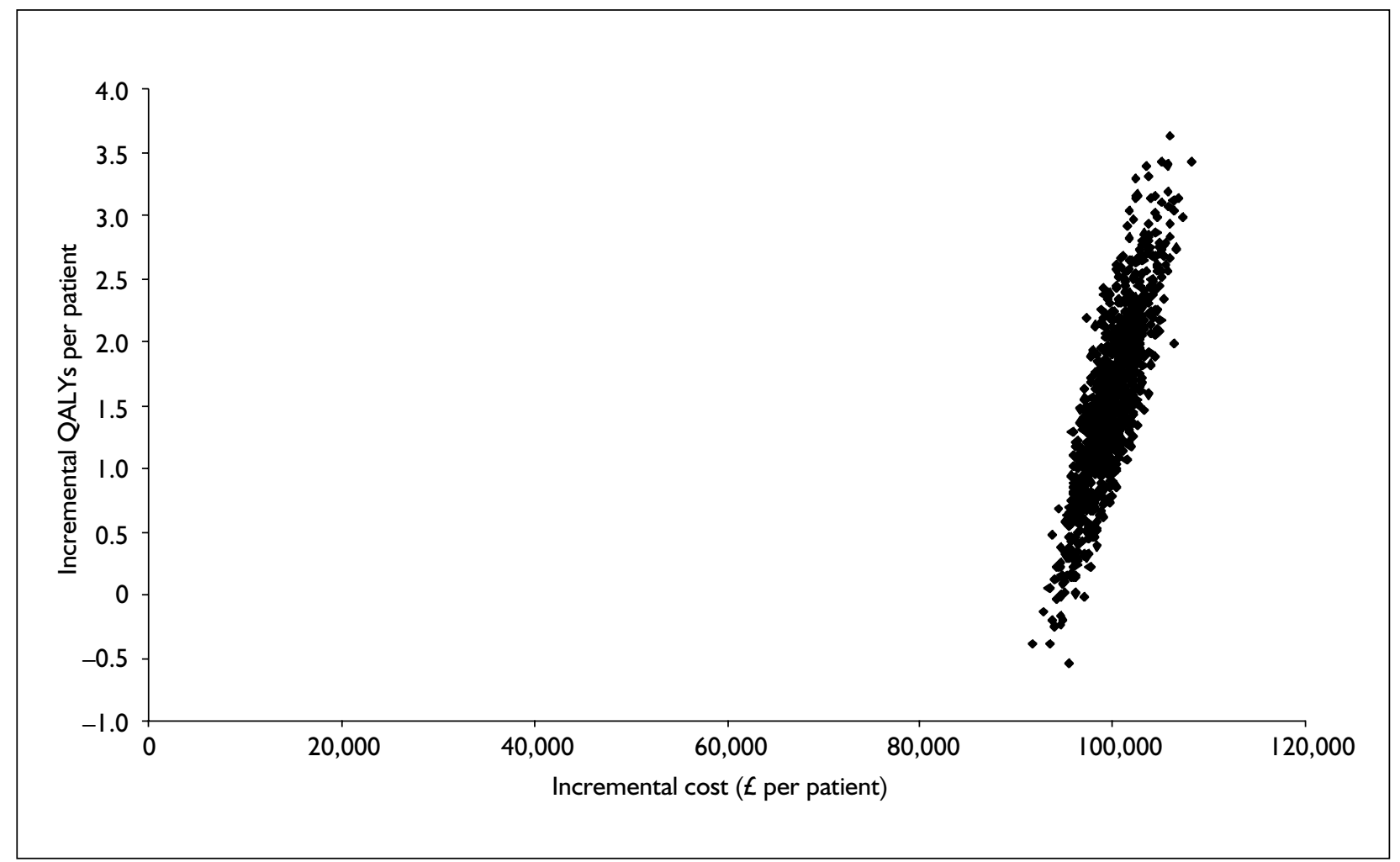


TABLE 55 Summarised baseline results from the LTCS model

\begin{tabular}{|c|c|c|c|}
\hline & LVAD & Medical therapy & Increment \\
\hline \multicolumn{4}{|l|}{ Costs per patient per period } \\
\hline Initial period & $£ 87,877$ & $£ 1,382$ & $£ 86,495$ \\
\hline Each subsequent period $(2-2 \mathrm{I})$ & $£ 4,192$ & $f 1,382$ & $€ 2,811$ \\
\hline Utility per patient & 0.93 & 0.70 & 0.23 \\
\hline \multicolumn{4}{|l|}{ Cohort (100 patients) } \\
\hline Total cost - undiscounted & $E||, 033,|3|$ & $£ 562,991$ & $£|0,470| 4 \mid$, \\
\hline Total QALYs - undiscounted & 114.60 & 50.07 & 64.53 \\
\hline Total cost - discounted & $f|0,675,64|$ & $£ 475,807$ & $£ 10,199,835$ \\
\hline Total QALYs - discounted & 104.19 & 44.41 & 59.78 \\
\hline ICER & & & $£ \mid 70,616$ \\
\hline
\end{tabular}

TABLE 56 One- and multi-way sensitivity analyses for LVADs as an LTCS for people with ESHF

\begin{tabular}{|c|c|c|c|c|c|}
\hline \multirow{3}{*}{$\begin{array}{l}\text { Parameter varied } \\
\text { Baseline } \\
\text { Upper and lower estimates of costs }\end{array}$} & \multirow[t]{2}{*}{ Base case } & \multicolumn{2}{|c|}{ Range of values tested } & \multicolumn{2}{|c|}{$\begin{array}{l}\text { Cost per QALY ( }(€) \\
\text { (range) }\end{array}$} \\
\hline & & & & $E \mid 7$ & 1,616 \\
\hline & \multicolumn{2}{|c|}{ See Table 49} & & $£ \mid 36,597$ & $£ \mid 90,283$ \\
\hline LVAD operation cost & $£ 36,986$ & $£ 29,589$ & $£ 44,383$ & $E \mid 58,242$ & $£ 182,989$ \\
\hline LVAD management cost & $£ 16,769$ & $E|3,4| 5$ & $£ 20,123$ & $E 164,300$ & $E \mid 76,932$ \\
\hline Medical management cost & $£ 5,526$ & $£ 4,421$ & $£ 16,769$ & $£ \mid 54,423$ & $£ \mid 72,207$ \\
\hline HeartMate/Novacor device cost & $£ 48,000$ & $£ 38,400$ & $£ 57,600$ & $E \mid 54,557$ & $E \mid 86,674$ \\
\hline MicroMed DeBakey device & $£ 48,000$ & $£ 28,000$ & $£ 42,000$ & $E|37| 6 \mid$, & $£ 160,579$ \\
\hline Jarvik device cost & $£ 48,000$ & $£ 32,758$ & $£ 49,137$ & $E \mid 45,120$ & $E \mid 72,518$ \\
\hline Improvement over REMATCH survival & $\mathrm{N} / \mathrm{A}$ & $40 \%$ & $60 \%$ & $£ 49,120$ & $£ 6 I, I 54$ \\
\hline $\begin{array}{l}\text { Improvement over REMATCH survival, } \\
\text { MicroMed DeBakey VAD device cost }\end{array}$ & $\mathrm{N} / \mathrm{A}$ & $40 \%$ & $60 \%$ & $£ 44,339$ & $£ 54,692$ \\
\hline Costs discount rate & $6 \%$ & $3 \%$ & $9 \%$ & $f 169,638$ & $E|7|, 656$ \\
\hline Benefits discount rate & $1.5 \%$ & $0 \%$ & $6 \%$ & $£ 167,059$ & $E|8|, 49 \mid$ \\
\hline Mean expert-based utilities & \multicolumn{3}{|c|}{ See Table 5 I } & $€|72,73|$ & \\
\hline
\end{tabular}

impact on cost-effectiveness. Similarly, even when medical management costs were four times those of LVAD management, there was little impact made on cost-effectiveness of LVADs as an LTCS.

There were insufficient data to populate the analysis of a second-generation device on any parameter except cost. However, some threshold analysis was attempted anchored to the firstgeneration survival curves. If a second-generation device was to show an improvement in survival over and above the first-generation values of $40-60 \%$ (using the $£ 35,000$ MicroMed Debakey VAD device cost), the cost per QALY ranges from $£ 44,339$ (with a $60 \%$ improvement) to $£ 54,692$ (40\% improvement). These figures approach acceptable cost-effectiveness thresholds and give an indication of the improvement which must be shown in future second-generation studies for their cost-effectiveness to be considered favourably.

For the LTCS model, only the most extreme sensitivity analysis results in an ICER that might generally be considered viable. These parameters have been best dealt with as an input parameter to the model (as has been addressed in this sensitivity analysis) rather than assigning a distribution. Therefore, for the LTCS model, the analysis has focused on a deterministic analysis (Table 55) rather than embarking on a stochastic analysis, as undertaken in the BTT model. 


\section{Budget impact/resource management issues}

The British Heart Foundation recently produced an updated breakdown of the NHS costs associated with heart failure based on 2000 data (see Table 57). ${ }^{162}$

The predominant cost component is hospitalisation, accounting for $60 \%$ of total costs. Severity of heart failure is classified according to the NYHA staging criteria. These rate patients as Class I-IV, with Class IV representing the greatest degree of debilitation, encompassing severe difficulties in daily activities and problems in breathing even at rest. Furthermore, the cost of heart failure increases exponentially with an increase in NYHA classification. In a study across four European countries by Berry and colleagues, ${ }^{163}$ costs of NYHA Class III patients were 2-4 times those of Classes I and II. The figures were even more marked for the costs of NYHA Class IV, which varied from 8 to 33 times those of Class I and II patients. Putting this in context, Class IV patients account for between $61 \%$ and $92 \%$ of heart failure costs. Of the nonmedical expenditure on heart failure (including productivity losses, carer support, welfare support ambulance transport and nursing care), few estimates have been reported.

The decision to place a patient on a waiting list for transplant is a multidisciplinary one driven by the age and underlying co-morbidity of the patient. Patients $>60$ years old or suffering from chronic current systemic infection are contraindicated for transplant. ${ }^{164}$ There are protocols in operation and a number of criteria must be met before a patient is eligible for waiting list status. The process generally works as follows: "Patients waiting for a heart or liver who are classified as urgent are given priority. This is because their life expectancy without a transplant can be measured in days or even hours. If there are no urgent patients on the waiting list, the

TABLE 57 Costs of heart failure to the NHS in $2000^{162}$

\begin{tabular}{|lcc|}
\hline & E million & $\%$ of total \\
\hline Primary care & 103.8 & 17 \\
Hospital inpatient care & 378.6 & 60 \\
Hospital day-case care & 0.45 & $<1$ \\
Hospital outpatient care & 51.25 & 8 \\
Outpatient investigations & 37.44 & 6 \\
Drugs & 54.08 & 9 \\
Total & 628.6 & 100 \\
\hline
\end{tabular}

organ is offered for patients on the non-urgent list who are nearest in age and blood group to the donor. The location of donor and recipient is also considered to minimise the delay between retrieving and transplanting organs" (UK Transplant).

Although there appears to be no adequate way of assessing unmet need (for the purposes of budget impact analyses) in the UK (Large S, Papworth Hospital NHS Trust: personal communication, 2003), using information from studies of incidence and prevalence, mortality data and waiting list data, the potential need and demand for LVADs within England and Wales were estimated (see Table 58 and the section 'End-stage heart failure and the needs and demands for healthcare' (p. 10) for a discussion of the methodology used for estimating need, demand and supply).

Although this analysis estimates that between 3000 and 34,000 people annually could benefit from treatment for ESHF, it is likely that actual need will be between 6000 and 8000 people. Although these figures are only an estimate, they give an idea of the potential for heart transplants were the availability of donor hearts unrestricted.

Furthermore, it is likely that the figures will increase when the potential expansion of the LVAD market is expanded to patients unsuitable for heart transplant. As noted above, the UK waiting list population is capped, as is likely the case in the USA, at a lower level than our projection and consequently reflective of supply as opposed to need.

The baseline analysis in the cost-effectiveness model put the annual increased burden on the

TABLE 58 UK projections (England and Wales) of need, demand and supply

\begin{tabular}{|lrrr|} 
& \multicolumn{3}{c|}{ Year } \\
\cline { 2 - 4 } & $\mathbf{1 9 9 5}$ & $\mathbf{2 0 0 3}$ & $\mathbf{2 0 0 0}$ \\
\hline Total need & 6,782 & 3,146 & $\mathbf{3 4 , 3 2 9}$ \\
Total demand & 893 & 414 & $\mathbf{4 0 2}$ \\
Unmet demand & 574 & 266 & $\mathbf{1 9 8}$ \\
Transplants (met demand) & $\mathbf{3 1 9}$ & $\mathbf{1 4 8}$ & $\mathbf{2 0 4}$ \\
Actual donor supply & 338 & 157 & $\mathbf{2 2 4}$ \\
Potential donor supply & 1,183 & 549 & 756 \\
Unmet need & 6,463 & 2,998 & 34,125 \\
Waiting list & 469 & 218 & $\mathbf{1 5 6}$ \\
Unrecognised need & 5,892 & 2,734 & 33,927 \\
\hline Figures in bold are from published sources and those in \\
italic were estimated. & & & \\
\end{tabular}


NHS at $£ 99,636$ per patient for the BTT model and $£ 101,998$ per patient for the LTCS model. This is an incremental cost to be incurred since no LVADs are currently provided on the NHS. If the use of the technology is expanded, first as a BTT for everyone on the waiting list $(n=156)$, the impact on the budget would increase to $£ 15.5$ million. Second, when the use of LVAD is not confined to transplant candidates but extended to wider definitions of ESHF, as in the LTCS scenario, the cost to the NHS will be significantly higher. It is estimated that the discounted costs will range from $£ 42.2$ million with a total demand of 414 people to $£ 321$ million when encompassing a total need of 3146 people. The costs of this technology will have to fall considerably before it achieves such widespread usage. Furthermore, any proposed expansion needs to consider issues of capacity. There are few experienced centres able to carry out LVAD operations, currently limited to four main UK specialist centres. The cost of training additional clinicians and support staff and equipping centres would also have to be taken into consideration and would require a considerable step change in funding to provide such a service within the NHS.

\section{Conclusion}

The economic evaluation has shown that LVADs do not appear to be a cost-effective use of NHS resources when used as either a BTT or an LTCS for people with ESHF given current UK thresholds of $£ 30,000$ per QALY. The baseline analyses produced a cost per QALY of $£ 65,242$ for BTT and $£ 170,616$ for LTCS, with substantial decreases in the costs associated with the device and its implantation and/or increased benefits to patients in terms of survival and QoL necessary to achieve acceptable ICERs and justify developing a service. Concerns about the declining availability of donor hearts for transplantation in the UK may also affect the nature of the service, limiting the possibilities for the use of LVADs as a BTT. Importantly, the results of the analysis should be viewed with caution owing to the uncertainties associated with the information on clinical effectiveness of the devices (particularly the second-generation LVADs), the costs of implantation and of the ongoing costs of medical management and the QoL gained by people with ESHF receiving the different treatment options. Additional research is required to allow a more accurate assessment of the costs and benefits of developing a service for the NHS.

\section{Discussion}

In an environment of diminishing donor hearts, "only a privileged minority receive transplants"165 and as a consequence the potential of this technology is huge. The number of transplants carried out in the UK has nearly halved in 10 years (from 302 in 1993 to 153 in 2002). ${ }^{164}$ The reasons for this decline are not clear and it may be that further research may explore the possibility of developing a strategy to increase organ donation that may compare favourably with the development of a service to provide LVADs to people with ESHF. Alternatives, such as xenotransplantation, are experimental ${ }^{166}$ and innovative therapies such as growing new heart tissue from cells ${ }^{167}$ are a long way off routine usage. Improving outcomes in BTT patients through the use of LVADs, although itself rewarding, will have limited effect on the use of LVADs as demand will be dependent on the availability of donor hearts. With the declining supply of organs for donation, it is unlikely that the role of LVADS as a BTT will grow. It is more likely that the future for these devices lies in their use as an LTCS. However, the economic evaluation undertaken for this report using the data available has shown that LTCS is not costeffective, except under the most extreme scenario (a 60\% improvement in survival over current firstgeneration devices). In effect, it appears that the benefits of LVADs as an LTCS in terms of lifeyears saved are arguably being outweighed by their prohibitive cost. Although these findings suggest a limited future for this technology, they should be seen within the context of the evaluation of this technology (i.e. patients with ESHF have a high mortality rate ${ }^{168}$ and limited treatment options), in addition to the key elements that determine the outcome of the costeffectiveness analyses and the uncertainties that remain within the models. This is particularly important for the model assessing LVADS as an LTCS for people with ESHF, where data on benefits and costs are relatively limited.

The effectiveness of LVADs as a BTT and as an LTCS for people with ESHF was assessed using survival of patients through the different stages of the clinical pathway used during their management. Unfortunately, survival data were limited. Only a small number of studies presented survival data for the different stages involved in managing ESHF. Studies often involved small numbers of patients, limiting the reliability of their findings. Also, as second-generation devices are relatively new, only a limited number of studies 
examined their effectiveness. In the economic evaluation of LVADs as a BTT for people with ESHF, it was evident that the cost-effectiveness was sensitive to the difference in the survival of patients with LVADs compared with medical management after transplant. It was evident from the systematic review of clinical effectiveness that patients receiving an LVAD appeared to have an improved survival compared with those patients receiving medical management. The extent of the benefit was difficult to judge, given some variation between studies and possible differences in prioritisation of the different patient groups for transplant (i.e. short waiting times for transplant for LVAD patients). Additional good-quality, longer term, controlled evaluations are required to establish the relationship further. In the evaluation of LVADs as an LTCS for patients with ESHF, the sensitivity analysis showed that the costeffectiveness was not sensitive to survival.

The availability of utilities for the comparator interventions during the different stages of the clinical pathway followed by the patients was a particular concern for the evaluation. Originally, it had been intended, with the collaboration of senior clinicians at LVAD centres within the UK, to solicit patient-based utilities from the LVAD population for this study. It had been hoped that it would be possible, through patients' routine appointments, to administer a simple questionnaire via a research nurse. Unfortunately, it became clear during the study that such an exercise was not feasible and regrettably it was abandoned and efforts were focused on other approaches. [There were reported recruitment problems to the EVAD study at Papworth and Harefield hospitals for LVAD patients and heart transplant comparison patients at Newcastle General Hospital. The EVAD team could not support our methodology, principally owing to their perception of the doubtful scientific or policy value of retrospective utility assessment in a small sample of patients, the time and effort involved in such an exercise and the degree to which it may complicate the EVAD study itself. The EVAD collaborators kindly offered access to their clinical experts to construct treatment pathways and utilities, and the NSCAG cost database in lieu (Caine N, Papworth Hospital NHS Trust, Cambridge: personal communication, 2003).] At the time of planning the patient-based exercise there were only around 19 surviving patients (60\% of 32 transplanted patients) at Papworth Hospital who had previously been on LVAD support. It was then planned to undertake a retrospective process to solicit the patients' QoL at three points: prior to placement of the LVAD, during LVAD support and their current status (giving us potentially 57 responses). [We had planned to administer both the EQ-5D instrument and an SG exercise following Moskowitz's scenarios with minor rewording with permission of the author (Moskowitz AJ, Columbia University: personal communication, 2003) facilitated by a booklet with laminated cards.] This was to be augmented with data from patients associated with the LVAD programme at the John Radcliffe Hospital, Oxford, but unfortunately it was not possible to obtain these data either.

As a consequence, the evaluation focused on a panel-based utility mapping exercise, which also has potential limitations. Mapping exercises are necessarily long drawn-out processes of validation and testing, which were impossible within the time horizon of this research. The final report on the process undertaken by Stein and colleagues in developing the utilities used is awaited with interest, so judgements can be made about the validity and the reliability of the process followed. It will be important to compare the utilities from the mapping exercise used within this evaluation with those from other studies, particularly those from the REMATCH study when published. It is understood that the utilities collected in the REMATCH trial used the EQ-5D instrument and the authors may also wish to compare these with utilities estimated via the algorithm developed by Brazier and colleagues ${ }^{169}$ to calculate utilities from the individual patient-level SF-36 scores, which are summarised by Rose and colleagues. ${ }^{111}$

Comparison of another study that performed a mapping exercise linking the NYHA classification with the EQ-5 $\mathrm{D}^{170}$ with the utilities presented in the systematic review of cost-effectiveness shows some variations. The utility scenarios examined by Kirsch and McGuire ${ }^{170}$ resulted in markedly lower values for ESHF (in their case NYHA Class IV patients, median utilities 0.284 to 0.372 ) than reported in other studies (see Chapter 4). Kirsch and McGuire ${ }^{170}$ expressed concerns with respect to decreasing proportionality over longer term survival after a period of getting one's "affairs in order" (a view shared by Waugh and Scott ${ }^{171}$ ). Other concerns with the utilities reported by different studies is that they do not measure all patients at each stage of the clinical pathway and that the time period at which the utilities were solicited do not cover the whole of the period for which the utilities are assigned. Usually values are mean (or median) utility estimates reflective of the point of assessment only, implying they could be 
inherently biased. For example, whereas those on a waiting list could be expected to grow steadily worse, the QoL profile or QoL over time for an LVAD recipient may indicate a poorer state of health while the patient adjusts to the device before improving steadily or utility in ESHF may be a function of time since diagnosis. This type of analysis has been evaluated in oncology using the Quality-adjusted Time Without Symptoms and Toxicity (Q-TWiST) approach. Future LVAD modelling research may wish to construct a similar quality-adjusted survival analysis or partitioned survival analysis models. ${ }^{172}$

Finally, there are concerns over the validity of extrapolating reported utilities from first generation devices to second-generation devices. Owing to the increasing preference for calculating cost per QALY by bodies such as NICE, coordinators of trials including second-generation devices are recommended to solicit patient-based utilities through instruments such as the EQ-5D, which confers only a very small additional burden on the patients (it takes about 3 minutes to complete). Strong representation has been made to those in the UK involved in undertaking research into the use of LVADs as an LTCS for people with ESHF to use the EQ-5D instrument in subsequent trials.

Despite these concerns about the utility data, the estimates for ESHF patients across a variety of definitions are generally consistent $(\sim 0.6)$, and there is a positive increment between LVAD support and ESHF in the available literature. Also, the utilities from the expert panel, and their similarity to the utilities for the patients receiving an LVAD in the study by Moskowitz and colleagues, ${ }^{138}$ provide a counter to the argument that patient-based condition-specific values are biased by a 'heroic intervention' effect (i.e. the patient is so relieved to be still alive that $\mathrm{s} / \mathrm{he}$ uplifts the utility score accordingly). Future researchers should also consider soliciting generically derived QALYS (as favoured by groups such as NICE) over condition-specific QALYS that tend to be provided by most of the literature.

As long-term follow-up data become available and studies utilise second- (and future-) generation devices, both the uptake and outcome of LTCS could be expected to improve. Generation of data will allow for robust comparisons between the costeffectiveness of the different indications. The economic evaluation of the REMATCH trial, where the HeartMate VE (first-generation) LVAD was used as an LTCS, together with patient-based utility estimates collected in that study (Moskowitz AJ, Columbia University: personal communication, 2003) are eagerly anticipated. Similarly, further data on second-generation LVADs (such as the Jarvik 2000, MicroMed DeBakey LVAD or HeartMateII LVAS) are awaited to populate economic models. Further research should also consider indirect or patient-borne costs, particularly if outpatient or home care is to be proposed.

As with other elements of the economic evaluation, cost data for the different aspects of treatment were difficult to obtain and comparisons provided some concerns about their reliability. Importantly, the sensitivity analyses undertaken for the BTT indication revealed that the combined cost of the procedures (LVAD device, assessment and implantation) had a significant influence on the overall cost-effectiveness. A previous evaluation by Christopher and Clegg ${ }^{121}$ of the use of LVADS as a BTT for ESHF found a cost per QALY of $£ 39,790$, markedly lower than that found in the current evaluations of LVADs as a BTT or an LTCS. The difference reflects the use in this evaluation of up-to-date LVAD specific costs which were far higher than those reported by Christopher and Clegg. ${ }^{121}$ The LVAD operation cost observed in the previous study was $£ 10,812$ (inflated) compared with $£ 36,986$ used in this evaluation. Similarly, the ongoing LVAD management costs were $£ 844$ (inflated) compared with the value used in this evaluation of $£ 16,769$. It is clear that these apparent differences in LVADrelated costs have pushed the BTT scenario beyond generally accepted cost-effectiveness norms and such costs would need to be controlled if the technology is to meet the current benchmark for acceptable cost-effectiveness within the UK. Also, there has been a marked fall in the published cost for heart transplantation reported in NHS Reference Costs. Christopher and Clegg ${ }^{121}$ reported the cost of heart transplantation at $£ 23,000$ in 1999 ; by 2003 it had fallen to $£ 14,114$ (2002, £16,712), although variation in this parameter has little impact on this scenario.

Uncertainties remain about the costs of the comparator interventions assessed in the economic evaluations. It proved impossible to obtain the ongoing costs of medical management from the UK and, as a consequence, US hospitalisation resource use data were used as a proxy for the relative cost of medical management compared with management with an LVAD. Such estimates are vulnerable to US hospital, health provider and FDA protocols. Sensitivity analyses undertaken 
suggested that changes in these variables had little impact on the outcomes of the economic evaluations. Further scenarios considered in the sensitivity analyses showed that when medical management of a waiting list or non-transplant candidate are substantial then the favourability of LVADs increases. The economic evaluations from the REMATCH study of LTCS in the USA and from the NSCAG EVAD study of BTT in the UK are eagerly awaited as they will provide additional information on benefits and costs.

Other factors may affect the cost of the use of LVADs in the UK in the future. The results in the economic evaluation of BTT in this report are within the inpatient setting. Several studies in the USA have examined the use of LVADs in the outpatient setting, where the costs of management may be lower. At present no studies have undertaken such an evaluation in the UK. Another possibility for reducing the high cost of the LVAD devices is the potential for their re-use after transplantation or death. Naturally such a strategy must overcome such issues as sterilisation, ethics, legalities and consent. ${ }^{173}$ Pacemakers, in particular, have a proven history of reuse. ${ }^{173}$ However, the devices are not currently reused. First-generation devices were designed with a lifespan of 1 year whereas second-generation devices were designed for up to 5 years (Banner N, Royal Brompton and Harefield NHS Trust: personal communication, 2003). Replacement surgery, moreover, may be more risky than the initial operation and is only done in an emergency situation. 


\section{Chapter 6 Discussion}

\section{Statement of principal findings}

The main findings of the systematic review of the clinical effectiveness of LVADs as a BTT, BTR and LTCS are summarised.

\section{Clinical effectiveness of LVADs as a BTT for people with ESHF}

Despite the relatively poor quality of the evidence, it was evident from the systematic review that LVADs as a BTT, compared with inotropic agents, optimum usual care or no care, appear to be beneficial for patients with ESHF when assessed using patient survival, functional status and QoL. When compared with inotropic agents, LVADs appeared to provide a benefit in patient survival that increased with the length of support (difference in actuarial survival: 1 month $3 \%$, 3 months 17\%) and extended beyond heart transplantation (difference in actuarial survival: 1 year 24\%, 4 years $30 \%$ ). Comparisons of the use of LVADs with usual care were less certain, with outcomes varying from no difference in survival to, or after, heart transplantation, to improved survival for LVADs patients to heart transplantation (survival difference: range $14-59 \%$ ) and post-transplantation (difference in actuarial survival: 1 and 2 years 100\%). Studies of LVADs which did not include a comparator were often the only evidence available, particularly for the new devices. In these studies, implantation of an LVAD provided support for up to 390 days, with as many as $70 \%$ of patients surviving to transplantation. Comparisons of different LVADs were limited. Only the HeartMate and Novacor LVADs were compared, with little difference in survival to transplantation. There was limited evidence assessing the effects of LVADs on the functional status and QoL of patients with ESHF bridged to heart transplantation. Patients supported by an LVAD appeared to have an improved functional status compared with those on usual care. Also, patients with an LVAD experienced an improvement in their QoL from before implantation of the device to the period during support. The use of LVADs is associated with risks of adverse events, with patients suffering mechanical device failures, bleeding, thromboembolic events, infections, reoperations and psychiatric conditions. Adverse event rates varied between different LVADs and studies and some caution should be taken in interpreting these results. With scarcity of evidence directly comparing the different LVADs, it is difficult, and perhaps inappropriate, to identify specific devices as the most clinically effective. However, the HeartMate LVAD is the only device that has evidence comparing it with several different alternatives, appearing to be more clinically effective than inotropic agents and usual care and as clinically effective as the Novacor device. Of the second-generation devices, the evidence suggests limited difference in the clinical effectiveness of the Jarvik 2000 and the MicroMed DeBakey LVADs. Although early in their development, studies of second-generation devices appear to show lower rates of adverse events, such as infection, bleeding and thromboembolism, that have affected the development and use of the first-generation devices. Some clinicians have questioned this finding, reporting difficulties with second-generation devices and thromboembolism compared with first-generation devices. Longer term outcomes are needed to ensure that these adverse events and other consequences associated with the non-pulsatile nature of the devices are accurately identified.

\section{Clinical effectiveness of LVADs as a BTR for people with ESHF}

Evidence of the clinical effectiveness of LVADs as a BTR for people with ESHF was limited to noncomparative observational studies, which were judged to be of poor methodological quality. The studies appeared to show that LVADs provided benefit in providing support for the patients until myocardial recovery. As there were no direct comparisons of different interventions, it is not possible to assess whether the LVADs are more effective than other alternatives or specific devices. No evidence was found to judge the effects of the devices on the QoL or functional status of patients. Limited information on adverse events was reported, although infections and bleeding were the main concerns.

\section{Clinical effectiveness of LVADs as an LTCS for people with ESHF} Although the nature and methodological quality of the evidence to assess the clinical effectiveness 
of LVADs as an LTCS for people with ESHF varied between the different devices, it was evident that LVADs provided benefits for patients in terms of improved survival, functional status and QoL. The REMATCH trial provided good-quality evidence that the HeartMate LVAD provided a statistically significant $48 \%$ reduction in the risk of death from any cause when compared with optimal medical management. Actuarial survival was significantly higher for patients with the HeartMate LVAD compared with optimal medical management at 1 year (52\% versus $25 \%)$ and 2 years (23\% versus $8 \%$ ) of follow-up. Importantly, improvements in 1 -year survival were evident for patients aged $<60$ years and those aged $60-69$ years. Less rigorous evidence for the Novacor, Toyobo and Jarvik 2000 devices showed relatively high survival (90\%), with patients supported for up to 4 years. Limited information of changes in patients' QoL and functional status suggested that patients experienced improvements on specific scales following implantation of the HeartMate and Jarvik LVADs. Inevitably there are adverse events associated with the use of LVADs, with device malfunctions, infection and bleeding associated with their use. The HeartMate LVAD was associated with twice as many serious adverse events than optimal medical management, with significantly higher rates of non-neurological bleeding and neurological dysfunction. Other adverse events affected the different treatment options, whether devices or drug treatment. Despite these adverse events, the benefits of these LVADs appear to outweigh limitations. Evidence of the clinical effectiveness of the different devices indicates that the HeartMate LVAD appears to be effective when compared with optimal medical management. For the second-generation devices, the early evidence suggests that the Jarvik 2000 shows promise; however, further research is needed to assess whether there will be any longterm consequences associated with the change in the nature of the circulatory support.

\section{Systematic review of the cost-effectiveness of LVADs for people with ESHF}

Although there are a number of simple costing studies, this review has shown there is relatively little published literature on the cost-effectiveness of LVADs. A number of the costing studies reported in this review had serious methodological flaws. Even those judged of 'higher quality' had caveats limiting applicability and generalisability to a UK population. Significant limitations of the studies in this review were their limited sample size and the lack of comparators against which to judge the significance of the reported costs. There was only one UK-based cost-utility analysis, conducted by Christopher and Clegg ${ }^{121}$ in 1999 . The authors populated their model with costs based on treatment protocols, data from individual Trust finance departments and utilities derived in the study by Moskowitz. Their analysis reports cost per QALY values at the boundary of acceptability given recent decision-making. Based on total treatment costs of both LVAD BTT support and heart transplantation, the discounted cost per QALY was $\sim £ 39,790$ (range from $£ 28,510$ to $£ 74,000)$. Christopher and Clegg ${ }^{121}$ used threshold analysis to calculate that an LVAD device and procedure cost of $£ 19,300$ would equate to a cost per QALY of $£ 20,000$ or less.

\section{Economic evaluation to assess the cost-effectiveness of LVADs for people with ESHF within the UK}

The economic evaluation has shown that neither LVAD indication considered, that is, BTT or LTCS, is a cost-effective use, by generally accepted thresholds in the UK, of scarce NHS resources. The baseline analysis for the use of LVADs as a BTT for ESHF shows that the cost per QALY for the HeartMate LVAD was $£ 65,242$, whereas the cost per QALY for LVADs as an LTCS for ESHF was $£ 170,616$. The BTT indication approaches cost-effectiveness only if the one-off costs associated with an LVAD were to fall considerably (by $>40 \%$ ). Unfortunately, even assuming this eventuality, the BTT indication use parallels an ever-decreasing supply of donor hearts, capping the ability of this innovative technology to yield widespread benefit. In the less restrictive indication, LTCS, where LVADs are not just given to patients awaiting transplantation, the analysis undertaken using the available data has shown that LTCS is not cost-effective. Although our analyses recognise the benefits in terms of survival and QoL, these are outweighed by associated increases in cost. Sensitivity analysis suggested that significant changes would be needed in costs or improvements in benefits for the device to near suggested cost-effectiveness thresholds.

Uncertainties remain particularly with the lack of trial data on LTCS, research on second-generation devices and ongoing costs of medical management. The REMATCH population was older and in NYHA Class IV, whereas the BTT studies comprised younger, healthier patients. Trial data establishing LVADs as LTCS in a younger, healthier population may lead to a more favourable economic argument. The use of expertbased utilities in the LTCS data is also controversial but our sensitivity testing has shown 
this not to be an influential factor in costeffectiveness at the margin. Further research is needed if LVADs are to achieve their potential to benefit to a substantial ESHF population.

\section{Strengths and limitations of the systematic review and economic evaluation}

This review has certain strengths, including the following:

- It is independent of vested interest.

- The systematic review brings together the evidence on the effectiveness of LVADs as a BTT, BTR and LTCS for people with ESHF, applying consistent methods of critical appraisal and presentation.

- The systematic review was guided by the principles for undertaking a systematic review. ${ }^{75}$ Prior to undertaking the systematic review and economic evaluation, the research methods were set out in a research protocol (Appendix 2), which was commented on by an advisory group. The protocol defined the research question, inclusion criteria, quality criteria, data extraction process and methods employed to undertake the different stages of the systematic review and economic evaluation.

- An advisory group has informed the systematic review and economic evaluation from its inception, through the development of the research protocol and completion of the report. The advisory group included a broad range of people associated with the technology within the UK and world-wide, such as clinicians, commissioners, public health professionals and academics. A list of the members of the advisory group, their conflicts of interest declared and the input provided at the different stages of the study are outlined in Appendix 32.

- Contact was made with the manufacturers of the different LVADs to inform them of the study, its aims and research methods, and to invite them to submit any additional information that may inform the systematic review and the economic evaluation. Unfortunately, only a limited number of these manufacturers responded, despite reminders being sent. All submissions are outlined in Appendix 4 and acknowledgement is made to those who took the time to provide the information. Information was considered for inclusion within the systematic review and economic evaluation using the research methods outlined and applied to all such information.
- Initially contact was made with a sample of authors of studies included within the systematic review where clarification of information was required. The response from these authors was very poor, with very few replying to these requests. As a consequence, it was decided not to continue with this process and to assume that any information about the methods of the study or results not reported were not undertaken in the study. ${ }^{174}$

- The key providers within England and Wales who currently implant LVADs and/or provide care for patients with ESHF, specifically Papworth Hospital in Cambridge, Harefield and Brompton Hospitals and John Radcliffe Hospital in Oxford, were contacted to try to obtain relevant information for the systematic review and economic evaluation. Obtaining relevant information was difficult. The fast changing nature of the technology, the limited number of patients undergoing such treatment annually and the importance of patient confidentiality have limited the availability of information on patients' outcomes of survival, QoL and costs. Despite these limitations, some information was provided on the costs and professional opinions of patient QoL.

- The quality assessment of studies included in the systematic review of the clinical effectiveness of LVADs for ESHF used a recognised tool that was thought to allow comparison across different study designs, essential for decisionmakers when comparing alternative treatments. ${ }^{78}$ The tool provided the opportunity to assess possible biases, whether inherent in the study design used or through deficiencies in the application and reporting of methods and other information. Only the components of the tool were judged and discussed, and no overall score was calculated as it may be misleading. ${ }^{174}$ The approach may be criticised as the standard criteria used may include components that could not be met when assessing this technology (e.g. blinding of patients and surgeons) and for not using separate lists of criteria specific to the different study designs, so that potentially goodquality observational studies are judged to be of poor quality compared with an unachievable 'gold standard'. However, comparison across the range of evidence on the different devices was important to this health technology assessment and an appreciation of the potential biases was key. As a consequence, it was felt that the advantages of the approach outweighed any disadvantages. 
- The economic evaluation provides the most up-to-date modelling of data on benefits of treatment and the costs within the UK, using data from relevant primary research to populate aspects of the clinical effectiveness of the technology, utilities from an independent expert panel and cost data from current service providers in the UK.

In contrast, there were certain limitations:

- Owing to differences in the design, duration, outcome measures and reporting of studies, synthesis of the included studies was through narrative review with no formal metaanalysis.

- Despite efforts, there was limited information provided by study authors, manufacturers and service providers that may have benefited the clarity of the information considered and the extent of the analyses undertaken.

- Different methods were used for the systematic review of clinical effectiveness and that of the cost-effectiveness of LVADs. Whereas the systematic review of clinical effectiveness undertook inclusion and exclusion decisions, extracted data and assessed quality using two independent reviewers, the systematic review of cost-effectiveness used one reviewer with decisions checked by a second reviewer. This may have reduced the robustness of the approach.

- The economic evaluation suffered from the inadequacies of the data available. Clinical effectiveness data varied in nature and methodological quality, making direct comparison difficult. Data on patient utilities were very limited and as a consequence experts from an independent panel used scenarios to provide estimates of patient utility. Costs were from one of the UK centres operating the NSCAG national service for ventricular assist devices and their ongoing research to evaluate the service, the EVAD study. As a consequence, the costs may be unrepresentative of the average within England and Wales. It was disappointing not to be able to obtain more information on clinical protocols, costs and QoL from the main clinical and research groups within the NHS in the UK. Concerns over historical data and academic confidentiality of current research data precluded access to such information. In addition, the limited information on the incidence and prevalence of ESHF renders estimates of the effect of developing a service for the NHS difficult.

\section{Other issues}

Several other issues should be taken into consideration in appraising the clinical and costeffectiveness of LVADs for ESHF:

- LVADs are a relatively new and fast changing technology, with limited diffusion within the UK and worldwide. As a consequence, the evidence base for the technology is relatively limited in scope and methodological quality, whether considering first- or second-generation devices. Studies are often retrospective, observational and non-comparative, focusing on specific conditions and narrowly defined patient groups. The pace of development looks set to persist, with new devices and guidance for their use and management continuing to emerge. Also, research has suggested new strategies for managing heart failure by combining LVADs with other technologies (e.g. drugs), ${ }^{175}$ and also possible limitations of combining with other technologies (e.g. pacemakers). With such change, it will be important to monitor and update this assessment regularly.

- Currently LVADs have tended to be limited to use within people suffering from ESHF and are often seen as treatment of last resort whether as a BTT, BTR or LTCS. However, it has been proposed that these devices might offer greater benefit to people if they were implanted before they progress to the severe state of ESHF, allowing people to survive for longer durations and to benefit from a better QoL. If so, it would suggest that these devices could be more costeffective, although much would depend upon the marginal benefits and costs offered over a different group of competing technologies. At present it is too early to make any judgement as to their future cost-effectiveness for these differing indications, as changes in their use would inevitably result in changes in the cost of competing alternatives. It would be inappropriate to compare these technologies through cost-effectiveness league tables.

- As technologies diffuse and competing alternatives emerge, so their cost usually decreases. It is likely that the cost of LVADs, whether first- or second-generation devices, will continue to fall. In addition, with few long-term studies, the benefits of using these devices may be undervalued. As research into their use continues, the clinical and cost-effectiveness may change.

- Often the limited nature of the research into such technologies is a consequence of their focus on meeting regulatory requirements. However, it may reflect the lack of research 
funding, experience of the clinical team and the constraints of using specific devices within particular patient groups limiting the scope of their research.

- It is a common concern in health technology assessment that conditions for research, such as patients, their condition and the setting, rarely reflect reality. It has been suggested that this is a concern for the assessment of LVADS for ESHF. Limitations in the evidence base limit the extent to which the findings of this research can be generalised. 



\section{Chapter 7 Conclusions}

\section{Implications for practice}

An important factor in planning NHS services for people with ESHF is whether the benefits of particular treatment options are sufficient to justify the costs of developing, and operating, the service. Current treatment options provide differing benefits to people with ESHF. Current medical management helps to provide some relief to patients but can only provide limited long-term benefit, with patients' condition declining with time. Heart transplantation has become an accepted form of treatment for patients at risk of dying from, or incapacitated by, severe or endstage heart failure. Unfortunately, the availability of heart donors continues to decline, severely limiting the use of this option to all but a very small proportion of people. The use of mechanical devices, such as LVADs, provides a treatment option with considerable promise. Currently, LVADs appear to be a clinically effective but not cost-effective treatment for bridging people with ESHF to heart transplantation. The availability of heart transplants renders this option of limited long-term benefit to patients and the NHS. The future of LVADs lies in their use as an LTCS for people with ESHF. At present the evidence for their clinical and cost-effectiveness is relatively limited, but more continues to emerge. The REMATCH trial has shown that the firstgeneration HeartMate device provides considerable survival benefit to people with ESHF. Case series and case studies in the UK, Germany and USA have shown that second-generation devices, such as the Jarvik 2000 and MicroMed DeBakey, appear to be clinically effective although the cost incurred limits their cost-effectiveness. Judging the acceptability of LVADs as an alternative to current treatment options will necessitate careful consideration of the costs of developing and operating the service against the consequences of other options. Limited numbers of LVADs are implanted in people with ESHF in England and Wales currently. Uncertainty remains about the extent of the need and demand for these devices within England and Wales, although there may be as many as 8000 people with the costs of providing such a service equating to as much as $£ 800$ million annually. Costs of setting up and developing the service would be in addition to this amount. Inevitably there may be some impact on other parties both within and outside the NHS, unless additional funding is available to fund any service developed. From a patient's perspective, LVADs, whether as a BTT, BTR or LTCS, provide benefit in terms of survival compared with the alternatives of medical management in a condition with a predictable and poor prognosis. Although the effect on QoL is less clear, the limitations of the early devices are progressively being addressed with people able to lead active and productive lives within the community. Given their condition and prognosis, people with ESHF are likely to demand LVADs as their only option, especially with the decreasing availability of donor organs for heart transplantation.

To meet any increased need for LVADS for ESHF, the NHS would need to ensure that there are adequate multidisciplinary teams available to undertake the procedures and support the patient through the long-term phase of managing life on the device. The availability of the different health professionals with appropriate training and experience in implanting and managing patients with LVADs for ESHF may be a limiting factor in developing the service. Inevitably there may be a considerable lead time to ensure that an adequately trained and funded service is put in place. Services would need to be located in specialist regional facilities to allow experienced teams to develop with the appropriate facilities.

\section{Recommendations for future research}

In undertaking the systematic review and economic evaluation, certain implications for research have become evident. These include the following:

- Despite studies on the epidemiology of heart failure, there appears to be little good-quality evidence on the epidemiology of ESHF. As a consequence, it is difficult to establish clearly the characteristics of the different patients who may require treatment and the possible need and demand for any health service. A systematic review of the epidemiology of ESHF should be 
undertaken to assess incidence and prevalence of ESHF and characteristics of the sufferers.

- The systematic review of the clinical and costeffectiveness of LVADs for ESHF revealed the limited nature and the poor quality of the evidence. Further research is needed to examine the clinical effectiveness of LVADs for people with ESHF, assessing patient survival, functional ability, QoL and adverse events. Although difficult to undertake with such fast changing technologies and with the different nature of the comparators, evaluations should be RCTs. These should focus on head to head comparisons of different technologies, whether different devices or usual medical care.

Importantly, these studies should encompass the breadth of patient groups that may benefit from these devices for the different indications. Importantly, studies should focus on the use of LVADS as a treatment for LTCS.

- Studies of the effectiveness of LVADs should include economic evaluations. It has become evident from this study that data for undertaking such studies are very limited. Data on QoL, utilities, resources and costs are not readily available. Changes in the technology, the clinical management of the condition and the patient groups benefiting have rendered the use of historical data problematic. As a consequence, studies should try to include appropriate data collection for assessment of clinical and cost-effectiveness.

- Studies should look at long-term outcomes beyond 2 years of follow-up. This will be particularly important with second-generation devices, where concerns have been raised about the effects of non-pulsatile devices.
Research in the UK is ongoing and will provide an important insight into the clinical and costeffectiveness of LVADs for ESHF, including:

- The NSCAG evaluation of the ventricular assist device programme in the UK (EVAD).

- Clinical evaluation of the Thoratec implantable ventricular assist device (IVAD) at Papworth Hospital NHS Trust.

- Jarvik 2000 Clinical Evaluation (evaluation of ventricular assist device - bridging to cardiac transplantation in the United Kingdom) at Newcastle upon Tyne Hospitals NHS Trust.

- A prospective longitudinal study comparing QoL and psychosocial adjustment for heart failure patients managed by medical therapy, an LVAD or by transplantation, led by Dr Claire Hallas at Royal Brompton and Harefield NHS Trust.

- Potential recovery of heart function in patients with advanced heart failure by treatment with a combination of LVAD, novel drug therapy and exercise training, led by Mr Asghar Khaghani at Royal Brompton and Harefield NHS Trust.

- A multicentre prospective trial of the HeartMate II LVAS for patients at risk of imminent death, by Mr Asghar Khaghani at Royal Brompton and Harefield NHS Trust.

- A multicentre trial of the VantraAssist device, led by Dr Steven Tsui at Papworth Hospital NHS Trust.

It will be important to monitor these and other studies not identified in study registers in the UK and worldwide, to include their results in revisions of this evaluation and to make further recommendations. 


\section{Acknowledgements}

$V^{2}$ arious people have contributed to the project and we are grateful for their help, including:

Dr Ken Stein, Senior Lecturer and Director, PenTAG, Peninsular Medical School, University of Exeter, UK.

Phil Pocock, Principal Statistician, UK Transplant, UK.

J Fawell, Papworth Hospital NHS Trust, Cambridge, UK.

Linda Durrant, SHTAC, University of Southampton, UK.

Kim Wherry, WIHRD, University of Southampton, UK.

Liz Hodson, WIHRD, University of Southampton, UK.

Dr Christine Clar, Independent Researcher, Germany.

Sian Thomas, Independent Researcher, Orkney Isles, UK.

Mariam Brazzelli, Research Fellow, HSRU, University of Aberdeen, UK.

Professor Norman Waugh, Department of Public Health, University of Aberdeen, UK.

Adam Lloyd, Fourth Hurdle Consulting Ltd, London, UK.

Dr Goro Matsumiya, Osaka University Graduate School of Medicine, Japan.

Dr Deereck Wheeldon, European Director Clinical Engineering, WorldHeart, UK.

Dr Charles Glanville, Thoratec Europe Ltd, Cambridge, UK.

Karen Brennan, Thoratec Europe Ltd, Cambridge, UK.

Dr P Jansen, Jarvik Heart Inc., New York, USA.

Dr Janine Meehan, Jarvik Heart Inc., New York, USA.

Dallas Anderson, President/CEO, MicroMed Technology Inc., Houston, Tx, USA.

Yosuke Kobayashi, Toyobo Co. Ltd, Osaka, Japan.

Dr Steve Livesey, Southampton General Hospital, UK.

Dr Bill Gutteridge, Medical Adviser, NSCAG, Department of Health, London, UK.

Dr N Banner, Royal Brompton and Harefield NHS Trust, London, UK.

Members of the Advisory Group who contributed to the report through comment on either the research protocol and/or a draft copy of the final report:

Professor S Ball, Academic Unit of Cardiovascular Medicine, Leeds General Infirmary, UK.

Professor Martin Buxton, Director, HERG, Brunel University, Middlesex, UK.

Ms Noreen Caine, Director of R\&D Unit, Papworth Hospital NHS Trust, Cambridge, UK.

Professor J Cleland, Professor of Cardiology, University of Hull, Castle Hill Hospital, Hull, UK.

Dr Mick Davies, Department of Cardiology, University Hospital Birmingham NHS Trust, The Queen Elizabeth Hospital, Birmingham, UK.

Mr Stephen Large, Consultant Cardiothoracic Surgeon, Director of VAD Services, Papworth Hospital NHS Trust, Cambridge, UK.

Dr S Ludgate, Medical Director, Medicines and Healthcare Products Regulatory Agency, London, UK.

Dr P McCarthy, Program Director, Heart Transplant and Mechanical Circulatory Support, Department of Thoracic and Cardiovascular Surgery, The Cleveland Clinic, Cleveland, $\mathrm{OH}$, USA.

Dr A Moskowitz, Co-Director, INCHOIR, Columbia University, New York, USA.

Professor John Pepper, Department of Cardiothoracic Surgery, Royal Brompton Hospital, London, UK.

Mr L Vale, Senior Research Fellow, Health Economics Research Unit, University of Aberdeen, UK.

Mr Stephen Westaby, Consultant Cardiothoracic Surgeon, Department of Cardiac Surgery, John Radcliffe Hospital, Oxford, UK.

Professor Sir MH Yacoub, Imperial College London and The Magdi Yacoub Institute.

NHLI at Heart Science Centre, Harefield, Middlesex, UK.

\section{Contributions of authors}

AJ Clegg (Senior Research Fellow and Director, SHTAC, University of Southampton) developed the research protocol; assisted in development of search strategy; assessed studies for inclusion in systematic review of clinical and cost-effectiveness; 
extracted data from, and quality assessed, included studies; synthesised evidence; assisted in development of economic model; collected data for economic evaluation; drafted and edited final report; and, project managed study. DA Scott (Senior Health Economist, Fourth Hurdle Consulting Ltd, London and Visiting Fellow, University of Southampton) developed the research protocol; assessed studies for inclusion in systematic review of cost-effectiveness; extracted data from, and quality assessed, included studies; synthesised evidence; developed the economic model; collected data for economic evaluation; drafted final report. E Loveman (Senior Researcher, SHTAC, University of Southampton) assessed studies for inclusion in systematic review of clinical effectiveness; extracted data from, and quality assessed, included studies; synthesised evidence; drafted report. J Colquitt (Senior Researcher, SHTAC, University of Southampton) assessed studies for inclusion in systematic review of clinical effectiveness; extracted data from, and quality assessed, included studies; synthesised evidence; drafted report. J Hutchinson (Health Economics Analyst, Fourth Hurdle Consulting Ltd, London) assessed studies for inclusion in systematic review of cost-effectiveness; extracted data from, and quality assessed included studies; synthesised evidence; collected data for economic evaluation; drafted report. P Royle (Senior Research Fellow, University of Aberdeen) developed literature search strategy; drafted report. J Bryant (Senior Researcher, SHTAC, University of Southampton) assessed studies for inclusion in systematic review of clinical and costeffectiveness; drafted report. AJ Clegg and DA Scott are guarantors for the study.

This report was commissioned by the NHS R\&D HTA Programme. The views expressed in this report are those of the authors and not necessarily those of the HTA programme or the Department of Health. Any errors are the responsibility of the authors. 


\section{References}

1. Wyngaarden JB, Smith LH, Bennett JC. Cecil textbook of medicine. London: WB Saunders; 1992.

2. Department of Health. National Service Framework for Coronary Heart Disease: Modern standards and service models. London: Department of Health; 2003. Chapter 6. Heart failure.

3. Ernhardt LF, Cline MJ. Organisation of the care of patients with heart failure. Lancet 1998;352:15-8.

4. Cleland JGF. Heart failure: a medical hydra. Lancet 1998;352:1-2.

5. Philbin EF. Comprehensive multidisciplinary programs for the management of patients with congestive heart failure. J Gen Intern Med 1999; 14:130-5.

6. Jones J, Brown A, Waugh N. Heart failure. Aberdeen: Scottish Health Purchasing Information Centre; 1998.

7. Souhami RL, Moxham J. Textbook of medicine. London: Churchill Livingstone; 1994.

8. Portner PM. Permanent mechanical circulatory assistance. In Baumgartner WA, Reitz B, Kasper E, Theodore J, editors. Heart and lung transplantation. Philadelphia: WB Saunders; 2002. pp. 531-57.

9. Taggart DP, Westaby S. Surgical management of heart failure. BMJ 1997;314:453-4.

10. Mahmood AK, Courtney JM, Akdis M, Reul H, Westaby S. Critical review of current left ventricular assist devices. Perfusion 2000; 15:399-420.

11. Abou-Awdi NL, Frazier OH. The HeartMate: a left ventricular assist device as a bridge to cardiac transplantation. Transplant Proc 1992; 24:2002-3.

12. Goldstein DJ, Oz MC, Rose EA. Implantable left ventricular assist devices. $N$ Engl J Med 1998; 339:1522-33.

13. Westaby S. The need for artificial hearts. Heart 1996;76:206.

14. Westaby S, Jin XY, Katsumata T, Taggart DP, Coats AJ, Frazier OH. Mechanical support in dilated cardiomyopathy: signs of early left ventricular recovery. Ann Thorac Surg 1997; 64:1303-8.

15. Rose EA, Goldstein DJ. Wearable long-term mechanical support for patients with end-stage heart disease: a tenable goal. Ann Thorac Surg 1996;61:399-402.
16. Aaronson KD, Mancini DM. Mortality remains high for outpatient transplant candidates with prolonged (>6 months) waiting list time. $\mathrm{J} \mathrm{Am} \mathrm{Coll}$ Cardiol 1999;33:1189-95.

17. Smith JA, Rabinov M, Bergin PJ, Williams TJ, Anderson J, Esmore DS. Mechanical circulatory support: a necessary adjunct to a cardiac transplantation service. Transplant Proc 1992;24:2004-5.

18. American College of Cardiology, American Heart Association. ACC/AHA pocket guidelines for evaluation and management of chronic heart failure in the adult. A report of the American College of Cardiology/American Heart Association Task Force on Practice Guidelines. www.acc.org/clinical/guidelines/failure/pdfs/ HF_pocketguide.pdf, 2002.

19. Coates AJS. The syndrome of heart failure. In Warrel DA, Cox TM, Firth JD, Benz EJ Jr, editors. Oxford: Oxford Univesity Press; 2003. pp. 832-45.

20. World Health Organization. International statistical classification of diseases and related health problems. Tenth revision. Geneva: World Health Organization; 1992.

21. Cowie MR, Wood DA. Incidence and aetiology of heart failure in the general population. Heart 1997;77:7.

22. Bowling A. Measuring disease: a review of diseasespecific quality of life measurement scales. Buckingham: Open University Press; 2001.

23. Task Force for the Diagnosis and Treatment of Chronic Heart Failure ESoC, Remme WJ, Wedberg K. Guidelines for the diagnosis and treatment of chronic heart failure. Eur Heart J 2001;22:1527-60.

24. Cowie MR, Wood DA, Coats AJ, Thompson SG, Poole-Wilson PA, Suresh V, et al. Incidence and aetiology of heart failure; a population-based study. Eur Heart J 1999;20:421-8.

25. Cleland JG, Khand A, Clark A. The heart failure epidemic: exactly how big is it? Eur Heart J 2001; 22:623-6.

26. Davis RC, Hobbs FD, Lip GY. ABC of heart failure. History and epidemiology. BMJ 2000;320:39-42.

27. Goldman JH, McKenna WJ. The epidemiology of heart failure secondary to coronary artery disease. Coron Artery Dis 1998;9:625-8.

28. Schocken DD, Arrieta MI, Leaverton PE, Ross EA. Prevalence and mortality rate of congestive heart failure in the United States. J Am Coll Cardiol 1992; 20:301-6. 
29. Parameshwar J, Shacknell MM, Richardson A, Poole-Wilson PA, Sutton GC. Prevalence of heart failure in three general practices in North West London. Br J Gen Pract 1992;42:289.

30. Garrison GE, McDonough JR, Hames CG, Stulb SC. Prevalence of chronic congestive heart failure in the population of Evans County, Georgia. Am J Epidemiol 1966;83:228-344.

31. Eriksson H, Svardsudd K, Larsson B, Ohlson LO, Tibblin G, Welin L, et al. Risk factors for heart failure in the general population: the study of men born in 1913. Eur Heart J 1989;10:647-56.

32. Ambrosio GB, Riva L, Casiglia E. Prevalence of congestive heart failure (CHF) in elderly. A survey from a population in Veneto Region. Acta Cardiol 1994;49:324-5.

33. Senni M, Tribouilloy CM, Rodeheffer RJ, Jacobsen SJ, Evans JM, Bailey KR, et al. Congestive heart failure in the community: trends in incidence and survival in a 10-year period. Arch Intern Med 1999;159:29-34.

34. Lloyd-Jones DM. The risk of congestive heart failure: sobering lessons from the Framingham Heart Study. Curr Cardiol Rep 2001;3:184-90.

35. Dominguez LJ, Parrinello G, Amato P, Licata G. Trends of congestive heart failure epidemiology: contrast with clinical trial results. Cardiologia 1999; 44:801-8.

36. Davies MK, Hobbs FDR, David RC, Kenkre JE, Roalfe AK, Hare R, et al. Prevalence of leftventricular systolic dysfunction and heart failure in the Echocardiographic Heart of England Screening study: a population based study. Lancet 2001;358:430-43

37. Phillips SJ, Whisnant JP, O'Fallon WM, Frye RL. Prevalence of cardiovascular disease and diabetes mellitus in residents of Rochester, Minnesota. Mayo Clin Proc 1990;65:344-59.

38. Johansson S, Wallander MA, Ruigomez A, GarciaRodriguez LA. Incidence of newly diagnosed heart failure in UK general practice. Eur J Heart Fail 2001;3:225-31.

39. Rywik SL, Wagrowska H, Broda G, Sarnecka A, Pytlak A, Polakowska M, et al. Heart failure in patients seeking medical help at outpatients clinics. Part I. General characteristics. Eur J Heart Fail 2000;2:413-21.

40. Krum H, Tonkin AM, Currie R, Djundjek R, Johnston CI. Chronic heart failure in Australian general practice: The Cardiac Awareness Survey and Evaluation (CASE) study. Med J Aust 2001; 174:439-44.

41. Morgan S, Smith H, Simpson I, Liddiard GS, Raphael H, Pickering RM, et al. Prevalence and clinical characteristics of left ventricular dysfunction among elderly patients in general practice setting: cross sectional survey. BMJ 1999; 318:368-72.

42. Davis RC, Hobbs FDR, Kenkre JE, Roalfe AK, Hare R, Lancashire RJ, et al. Prevalence of left ventricular systolic dysfunction and heart failure in high risk patients: community based epidemiological study. BMJ 2002;325:1156-8.

43. McCallum J, Doherty J, Marshall H. Prevalence of heart failure in a west of Scotland general practice. Br J Cardiol 2001;8:179-80 and 182.

44. Epstein FH, Ostrander LD, Johnson BC. Epidemiological studies of cardiovascular disease in a total community - Tecumseh, Michigan. Ann Intern Med 1965;62:1170-85.

45. Fuat A, Hunging APS, Murphy JJ. Barriers to accurate diagnosis and effective management of heart failure in primary care: qualitative study. BMJ 2003;326:1-6.

46. Cleland JG, Gemmell I, Khand A, Boddy S. Is the prognosis of heart failure improving? Eur J Heart Fail 1999;1:229-41.

47. McMurray J, McDonagh T, Morrison C, Dargie HJ. Trends in hospitalization for heart failure in Scotland 1980-1990. Eur Heart J 1993; 14:1158-62.

48. Cowie MR, Wood DA, Coats AJ, Thompson SG, Suresh V, Poole-Wilson PA, et al. Survival of patients with a new diagnosis of heart failure: a population based study. Heart 2000;83:505-10.

49. Cleland JGF, Cohen-Solal A, Aguilar JC, Dietz R, Eastaugh J, Follath F, et al. Management of heart failure in primary care (the IMPROVEMENT of Heart Failure Programme): an international survey. Lancet 2002;360:1631-9.

50. Cleland JGF, Swedberg K, Follath F, Komajda M, Cohen-Solal A, Aguilar JC, et al. The Euroheart Failure survey programme - a survey on the quality of care among patients with heart failure in Europe. Eur Heart J 2003;24:442-63.

51. Rengo F, Acanfora D, Trojano L, Furgi G, Picone C, Iannuzzi GL, et al. Congestive heart failure in the elderly. Arch Gerontol Geriatr 1996;23:201-23.

52. Tavazzi L, Ferrari R, Opasich C. Survey on heart failure in Italian hospital cardiology units. Results of the SEOSI study. Eur Heart J 1997;18:1457-64.

53. Albanese MC, Plewka M, Gregori D, Fresco C, Avon G, Caliandro D, et al. Use of medical resources and quality of life of patients with chronic heart failure: a prospective survey in a large Italian community hospital. Eur J Heart Fail 1999;1:411-7.

54. Office for National Statistics. Mid-2002 Population Estimates: England and Wales; estimated resident population by single year of age and sex. URL: http://www.statistics.gov.uk/statbase/ssdataset.asp? $\operatorname{vlnk}=7024 \&$ More $=\mathrm{Y}$ (accessed 2 March 2004). 
55. Kazzaz N. Left ventricular assist devices. J Ark Med Soc 2001;98:178-9.

56. Evans RW. Cardiac replacement: estimation of need, demand and supply. In Rose EA, Stevenson LW, editors. Management of end-stage heart disease. Philadelphia, PA: Lippincott-Raven; 1998. pp. 13-24.

57. World Health Organization. Manual of the international statistical classification of diseases, injuries, and causes of death - version 9. Geneva: World Health Organization; 1977.

58. Office for National Statistics. Mortality statistics: cause. Series DH2, No. 28. Review of the Registrar General on deaths by cause, sex and age in England and Wales, 2001. London: HMSO; 2002.

59. Garg R, Yusuf S. For the Collaborative Group on ACE Inhibitor Trials. Overview of randomised trials of angiotensin-converting enzyme inhibitors on mortality and morbidity in patients with heart failure. JAMA 1995;273:1450-6.

60. Aaronson PI, Ward JPT, Wiener CM, Schulman SP, Gill JS. The cardiovascular system at a glance. Oxford: Blackwell Science; 2002.

61. Cohn JN, Archibald DG, Ziesche S, Franciosa JA, Harston WE, Tristan FE, et al. Effect of vasodilator therapy on mortality in chronic congestive heart failure: results of a Veterans Administration Cooperative Study. N Engl J Med 1986;314:1547-52.

62. Klein L, O'Connor CM, Gattis WA, Zampino M, de Luca L, Vitarelli A, et al. Pharmacologic therapy for patients with chronic heart failure and reduced systolic function: review of trials and practical considerations. Am J Cardiol 2003;91 (9A): $18 \mathrm{~F}-40 \mathrm{~F}$.

63. Goldstein DJ. Intracorporeal support: thermoc cardiosystems ventricular assist devices. In: Goldstein DJ, Oz MC, editors. Cardiac assist devices. New York: Futura Publishing; 2000. pp. 307-22.

64. Ramasamy N, Vargo RL, Kormos RL, Portner PM. Intracorporeal support: the Novacor left ventricular assist system. In Goldstein DJ, Oz MC, editors. Cardiac assist devices. New York: Futura Publishing; 2000. pp. 323-40.

65. Jett G, Lazzara R. Extracorporeal support: the ABIOMED BVS 5000. In Goldstein DJ, Oz MC, editors. Cardiac assist devices. New York: Futura Publishing Company; 2000. pp. 235-50.

66. Pennington DG, Oaks TE, Lohmann DP. Extracorporeal support: the Thoratec device. In Goldstein DJ, Oz MC, editors. Cardiac assist devices. New York: Futura Publishing; 2000. pp. 251-62.

67. Loebe M, Kaufman F, Hetzer R. Extracorporeal support: the Berlin Heart. In Goldstein DJ, Oz MC, editors. Cardiac assist devices. New York: Futura Publishing; 2000. pp. 275-90.
68. Derose J, Jarvik R. Axial flow pumps. In Goldstein DJ, Oz MC, editors. Cardiac assist devices. New York: Futura Publishing; 2000. pp. 359-74.

69. Noon GP, Morley D, Irwin S, DeBakey ME. The DeBakey ventricular assist device. In Goldstein DJ, $\mathrm{Oz}$ MC, editors. Cardiac assist devices. New York: Futura Publishing; 2000. pp. 375-86.

70. Rose EA, Moskowitz AJ, Packer M, Sollano JA, Williams DL, Tierney AR, et al. The REMATCH trial: rationale, design, and end points. Randomized evaluation of mechanical assistance for the treatment of congestive heart failure. Ann Thorac Surg 1999;67:723-30.

71. Clegg A, Bannigan K. Shifting the balance of opinion: Randomised controlled trials in occupational therapy. Br J Occup Ther 1997; 60:510-2.

72. Cochrane A. Effectiveness and efficiency. Random reflections on the Health Service. London: Nuffield Provincial Hospitals Trust; 1972.

73. Muir Gray J. Evidence-based health care. How to make health policy and management decisions. London: Churchill Livingstone; 1997.

74. Waugh NR. Grading of scientific evidence. Health Bull 1999;57:53-63.

75. CRD. Undertaking systematic reviews of research on effectiveness. Report No. 4. York: Centre for Reviews and Dissemination; University of York; 2001.

76. Juni P, Altman DG, Egger M. Assessing the quality of randomised controlled trials. In Egger M, Davey Smith G, Altman D, editors. Systematic reviews in health dare: meta-analysis in context. London: BMJ Publishing Group; 2001. pp. 87-108.

77. Thomas BH, Ciliska D, Dobbins M, Micucci S. A process for systematically reviewing the literature: Providing the research evidence for public health nursing interventions. Worldviews on Evidence-Based Nursing 2004;1(3):176-84.

78. Deeks J, Dinnes J, D'Amico R, Sowden A, Sakarovitch C, Song F, et al. Evaluating nonrandomised intervention studies. Health Technol Assess 2003;7(27).

79. Drummond MF, O'Brien B, Stoddart GL, Torrance GW. Methods for the economic evaluation of health care programmes. Oxford: Oxford University Press; 1997.

80. El Banayosy A, Arusoglu L, Kizner L, Tenderich G, Minami K, Inoue K, et al. Novacor left ventricular assist system versus HeartMate vented electric left ventricular assist system as a long-term mechanical circulatory support device in bridging patients: a prospective study.J Thorac Cardiovasc Surg 2000;119:581-7. 
81. Massad MG, McCarthy PM, Smedira NG, Cook DJ, Ratliff NB, Goormastic M, et al. Does successful bridging with the implantable left ventricular assist device affect cardiac transplantation outcome? J Thorac Cardiovasc Surg 1996;112:1275-83.

82. Aaronson KD, Eppinger MJ, Dyke DB, Wright S, Pagani FD. Left ventricular assist device therapy improves utilization of donor hearts. $\mathrm{J} \mathrm{Am} \mathrm{Coll}$ Cardiol 2002;39:1247-54.

83. Bank AJ, Mir SH, Nguyen DQ, Bolman RM, Shumway SJ, Miller LW, et al. Effects of left ventricular assist devices on outcomes in patients undergoing heart transplantation. Ann Thorac Surg 2000;69:1369-74.

84. Grady KL, Meyer P, Mattea A, White-Williams C, Ormaza S, Kaan A, et al. Improvement in quality of life outcomes 2 weeks after left ventricular assist device implantation.J Heart Lung Transplant 2001;20:657-69.

85. Frazier OH, Rose EA, Macmanus Q, Burton NA, Lefrak EA, Poirier VL, et al. Multicenter clinical evaluation of the HeartMate 1000 IP left ventricular assist device. Ann Thorac Surg 1992; 53:1080-90.

86. Frazier OH, Macris MP, Myers TJ, Duncan JM, Radovancevic B, Parnis SM, et al. Improved survival after extended bridge to cardiac transplantation. Ann Thorac Surg 1994;57:1416-22.

87. Trachiotis GD, D'Amico C, Johnston TS, Smith AL, Lutz JF, Vega JD, et al. Short- or long-term support with left ventricular assist device (LVAD) provides an effective bridge to transplantation. J Congest Heart Fail Circul Support 2000;1:119-22.

88. Masai T, Shimazaki Y, Kadoba K, Miyamoto Y, Sawa Y, Yagura A, et al. Clinical experience with long-term use of the Toyobo left ventricular assist system. ASAIO J 1995;41:M522-M525.

89. Holman WL, Roye GD, Bourge RC, McGiffin DC, Iyer SS, Kirklin JK. Circulatory support for myocardial infarction with ventricular arrhythmias. Ann Thorac Surg 1995;59:1230-1.

90. May DR, Adams MA. Ventricular assist devices. A bridge to cardiac transplantation. AORN J 1987; 46:633-6.

91. Noon GP, Morley DL, Irwin S, Abdelsayed SV, Benkowski RJ, Lynch BE. Clinical experience with the MicroMed DeBakey ventricular assist device. Ann Thorac Surg 2001;71:S133-S138.

92. Wieselthaler GM, Schima H, Dworschak M, Quittan M, Nuhr M, Czerny M, et al. First experiences with outpatient care of patients with implanted axial flow pumps. Artif Organs 2001; 25:331-5

93. Potapov EV, Loebe M, Nasseri BA, Sinawski H, Koster A, Kuppe H, et al. Pulsatile flow in patients with a novel nonpulsatile implantable ventricular assist device. Circulation 2000;102:III183-III187.

94. Frazier OH, Myers TJ, Jarvik RK, Westaby S, Pigott DW, Gregoric ID, et al. Research and development of an implantable, axial-flow left ventricular assist device: the Jarvik 2000 Heart. Ann Thorac Surg 2001;71:S125-S132.

95. Westaby S, Frazier OH, Beyersdorf F, Saito S, Siegenthaler MP, Pigott DW, et al. The Jarvick 2000 heart. Clinical validation of the intraventricular position. Eur J Cardiothorac Surg 2002;22:228-32.

96. Westaby S, Jarvik R, Freeland A, Pigott D, Robson $\mathrm{D}$, Saito S, et al. Postauricular percutaneous power delivery for permanent mechanical circulatory support. J Thorac Cardiovasc Surg 2002;123:977-83.

97. Frazier OH, Myers TJ, Gregoric ID, Khan T, Delgado R, Croitoru M, et al. Initial clinical experience with the Jarvick 2000 implantable axial-flow left ventricular assist system. Circulation 2002;105:2855-60.

98. Westaby S, Banning AP, Saito S, Pigott DW, Jin XY, Catarino PA, et al. Circulatory support for longterm treatment of heart failure: experience with an intraventricular continuous flow pump. Circulation 2002; 105:2588-91.

99. Siegenthaler MP, Martin J, van de Loo A, Doenst T, Bothe W, Beyersdorf F. Implantation of the permanent Jarvick 2000 left ventricular assist device. J Am Coll Cardiol 2002;39:1764-72.

100. Frazier OH, Myers TJ, Westaby S, Gregoric ID. Use of the Jarvik 2000 left ventricular assist system as a bridge to heart transplantation or as destination therapy for patients with chronic heart failure. Ann Surg 2003;237:631-7.

101. Wieselthaler GM, Schima H, Lassnigg AM, Dworschak M, Pacher R, Grimm M, et al. Lessons learned from the first clinical implants of the DeBakey ventricular assist device axial pump: a single center report. Ann Thorac Surg 2001; 71:S139-S143.

102. Wieselthaler GM, Schima H, Hiesmayr M, Pacher R, Laufer G, Noon GP, et al. First clinical experience with the DeBakey VAD continuousaxial-flow pump for bridge to transplantation. Circulation 2000;101:356-9.

103. Wieselthaler GM, Schima H, Lassnigg A, Pacher R, Ovsenk T, Laufer G, et al. Die DeBakey VADAxialpumpe: Erste klinische Erfahrungen mit einer neuen Generation von implantierbaren, nonpulsatilen Blutpumpen als LangzeitUberbruckung bis zur Transplantation. [The DeBakey VAD axial flow pump: first clinical experience with a new generation of implantable, nonpulsatile blood pumps for long-term support prior to transplantation]. Wien Klin Wochenschr 1999;111:629-35. 
104. Marelli D, Laks H, Amsel B, Jett GK, Couper G, Ardehali A, et al. Temporary mechanical support with the BVS 5000 assist device during treatment of acute myocarditis. J Card Surg 1997;12:55-9.

105. Kjellman UW, Hallgren P, Bergh CH, Lycke J, Oldfors A, Wiklund L. Weaning from mechanical support in a patient with acute heart failure and multiple sclerosis. Ann Thorac Surg 2000; 69:628-30.

106. Pietsch L, Laube H, Baumann G, Konertz W. Recovery from end-stage ischemic cardiomyopathy during long-term LVAD support. Ann Thorac Surg 1998;66:555-7.

107. Joharchi MS, Neiser U, Lenschow U, Schubert J, Kienast W, Noeldge-Schomburg G, et al. Thoratec left ventricular assist device for bridging to recovery in fulminant acute myocarditis. Ann Thorac Surg 2002;74:234-5.

108. Ueno T, Bergin P, Richardson M, Esmore DS. Bridge to recovery with a left ventricular assist device for fulminant acute myocarditis. Ann Thorac Surg 2000;69:284-6.

109. Nakatani T, Sasako Y, Kobayashi J, Komamura K, Kosakai Y, Nakano K, et al. Recovery of cardiac function by long-term left ventricular support in patients with end-stage cardiomyopathy. ASAIOJ 1998;44:M516-M520.

110. Noda H, Takano H, Taenaka Y, Nakatani T, Umezu M, Kinoshita M, et al. Treatment of acute myocardial infarction with cardiogenic shock using left ventricular assist device. Int J Artif Organs 1989;12:175-9.

111. Rose EA, Gelijns AC, Moskowitz AJ, Heitjan DF, Stevenson LW, Dembitsky W, et al. Long-term mechanical left ventricular assistance for end-stage heart failure. N Engl J Med 2001;345:1435-43.

112. El Banayosy A, Arusoglu L, Kizner L, Morshuis M, Tenderich G, Pae WE Jr, et al. Preliminary experience with the LionHeart left ventricular assist device in patients with end-stage heart failure. Ann Thorac Surg 2003;75:1469-75.

113. Dohmen PM, Laube H, de Jonge K, Konertz W. A reliable test to detect impending pump failure during long-term support on the Novacor N100 left ventricular assist system. Texas Heart Inst J 2001;28:139-41.

114. Seki T, Kitamura S, Kawachi K, Fukutomi M, Kobayashi S, Kawata T, et al. Efficacy and limitation of a left ventricular assist system in a patient with dilated cardiomyopathy accompanying multi-organ dysfunction. J Cardiovasc Surg (Torino) 1995;36:147-51.

115. Dohmen PM, Laube H, de Jonge K, Baumann G, Konertz W. Mechanical circulatory support for one thousand days or more with the Novacor N100 left ventricular assist device. J Thorac Cardiovasc Surg 1999;117:1029-30.
116. Drummond MF, Jefferson TO, on behalf of the BMJ Economic Evaluation Working Party. Guidelines for authors and peer reviewers of economic submissions to the BMJ. BMJ 1996; 313:275-83.

117. Moskowitz AJ, Rose EA, Gelijns AC. The cost of long-term LVAD implantation. Ann Thorac Surg 2001;71:S195-S198.

118. Federal Drug Administration. FDA approves heart assist pump for permanent use. URL:

http://www.fda.gov/bbs/topics/NEWS/2002/ NEW00851.html

119. Arabia FA, Smith RG, Jaffe C, Wild JC, Rose DS, Nelson RJ, et al. Cost analysis of the Novacor left ventricular assist system as an outpatient bridge to heart transplantation. ASAIO J 1996; 42:M546-M549.

120. CETS. Implantable ventricular assist devices: should they be used in Quebec? Montréal: Conseil d'Évaluation des Technologies de la Santé du Québec; 2000.

121. Christopher F, Clegg A. Left ventricular assist devices (LVADs) for end stage heart failure. Southampton: Wessex Institute for Health Research and Development; 1999. Development and Evaluation Committee No. 103.

122. Cloy MJ, Myers TJ, Stutts LA, Macris MP, Frazier OH. Hospital charges for conventional therapy versus left ventricular assist system therapy in heart transplant patients. ASAIOJ 1995;41:M535-M539.

123. Couper GS, Dekkers RJ, Adams DH. The logistics and cost-effectiveness of circulatory support: advantages of the ABIOMED BVS 5000. Ann Thorac Surg 1999;68:646-9.

124. Gelijns AC, Richards AF, Williams DL, Oz MC, Oliveira J, Moskowitz AJ. Evolving costs of longterm left ventricular assist device implantation. Ann Thorac Surg 1997;64:1312-9.

125. Loisance D, Benvenuti C, Lebrun T, Leclerc A, Tarral A, Sailly JC. Cost and cost effectiveness of the mechanical and pharmacologic bridge to transplantation. ASAIO Trans 1991;37: M125-M127.

126. Mehta SM, Aufiero TX, Pae W-EJ, Miller CA, Pierce WS. Mechanical ventricular assistance: an economical and effective means of treating endstage heart disease. Ann Thorac Surg 1995; 60:284-90.

127. Morales DL, Catanese KA, Helman DN, Williams MR, Weinberg A, Goldstein DJ, et al. Six-year experience of caring for forty-four patients with a left ventricular assist device at home: safe, economical, necessary. J Thorac Cardiovasc Surg 2000;119:251-9. 
128. Moskowitz AJ, Williams DL, Tierney A, Levitan RG, Zivin J, Gelijns AC. Economic considerations of left ventricular assist device implantation. In Goldstein DJ, Oz MC, editors. Cardiac assist devices. New York: Futura Publishing; 2000. pp. 183-91.

129. Oz MC, Grewal R, Gelijn A. Cost considerations for long-term mechanical circulatory support. ASAIO J 1997;43:268-70.

130. Schiller W, Reichart B. Cost-analysis of treatment with the portable, NOVACOR-left-heart-assistsystem. In Szucs TD, Haverich A, Odar J, editors. Economics of surgical procedures. Heidelberg: JA Barth; 2000. pp. 33-8.

131. Skinner JL, Harris C, Aaron MF, McGiffin DC, Kirklin JK, Bourge RC, et al. Cost-benefit analysis of extended antifungal prophylaxis in ventricular assist devices. ASAIOJ 2000;46:587-9.

132. Christensen DM, Frazier PA, Messner ER, Pae WE Jr, Silber DH, Sun B, et al., Save me the money: a cost comparison of inpatient vs. outpatient care of left ventricular assist device patients. 48th Annual Scientific Session of the American College of Cardiology, New Orleans, Louisiana, USA, March 7-10, 1999. J Am Coll Cardiol 1999;33(2 Suppl A):204A.

133. Kolbye A, Sander-Jensen KM, Aldershvile J, Hansen PB, Kjersem AM, Nielsen KS, et al. Okonomiske betragtninger ved implementering af det mekaniske hjerte, "HeartMate". [Economic aspects of implementation of the mechanical heart, "HeartMate"]. Ugeskr Laeger 2000; 162:3722-5.

134. Miller LW., Magovern G, Shannon RP, Mehra M, Van Meter C, Huiling L, et al. Costs of LVAD implantation: lessons from the REMATCH trial. In American Heart Association Scientific Sessions, November 17-20, 2002.

135. Mir SH, Kshetty VR, Kubo SH, Bolman RM, Park S, Francis GS, Bank AJ. Effects of the HeartMate left ventricular assist device on clinical outcomes and cost in status 1 heart transplantation patients. 70th Scientific Sessions of the American Heart Association, Orlando, Florida, USA, November 9-12, 1997. Circulation 1997;96 (8 Suppl):I367-8.

136. Petty M, Kshetty VR, Ormanza S, Bolman RM. Daily costs reduced for status 1 heart transplant (HT) patients following bridge with HeartMate left ventricular assist device (LVAD). Seventeenth Annual Meeting and Scientific Sessions of the International Society for Heart and Lung Transplantation, London, England, UK, 2-5 April 1997.J Heart Lung Transplant. 1997;16:57.

137. Schulze B, Tenderich G, Hansky B, El Banayosy A, Arusoglu L, Deyerling W, et al. In hospital costs of left ventricular assist device (Novacor) in comparison to different therapeutical strategies in end stage heart disease. XXVII Congress of the
European Society for Artificial Organs in Co-operation with the European Society for Engineering and Medicine, Lausanne, Switzerland, 31 August2 September, 2000. Int J Artif Organs 2000; 23:553.

138. Moskowitz AJ, Weinberg AD, Oz MC, Williams DL. Quality of life with an implanted left ventricular assist device. Ann Thorac Surg 1997;64:1764-9.

139. Havranek E, McGovern K, Weinberger J, Brocato A, Lowes B, Abraham WT. Patient preferences for heart failure treatment: utilities are valid measures of health related quality of life in heart failure. J Card Fail 1999;5:85-91.

140. Lewis E, Johnson P, Johnson W, Collins C, Griffin L, Stevenson LW. Preferences for quality of life or survival expressed by patients with heart failure. J Heart Lung Transplant 2001;20: 1016-24.

141. Moskowitz AJ, Williams DL, Tierney A, Levitan RG, Zivin J, Gelijns AC. Economic consideration of left ventricular assist device implantation. In Goldstein DJ, Oz MC, editors. Cardiac assist devices. New York: Futura; 2000.

142. Jefferson T, Demicheli V, Vale L. Quality of systematic reviews of economic evaluations in health care. JAMA 2002;287:2809-12.

143. Ryan M, Scott D, Reeves C, Bate A, Russell E, Napper M, et al. Eliciting public preferences for healthcare: a systematic review of techniques. Health Technol Assess 2001;5(5).

144. McGregor M. Implantable ventricular assist devices: is it time to introduce them in Canada? Can J Cardiol 2000;16:629-40.

145. Jouveshomme S, Baffert S, Fay A-F. Couer Artificiel (III). Dossier CEDIT. Paris: Assistance Publique Hopitaux de Paris; 1998.

146. Frazier OH, Rose EA, McCarthy P, Burton NA, Tector A, Levin H, et al. Improved mortality and rehabilitation of transplant candidates treated with a long-term implantable left ventricular assist system. Ann Surg 1995;222:327-36.

147. Evans RW. Economic impact of mechanical cardiac assistance. Prog Cardiovasc Dis 2000;43:81-94.

148. CETS. Hemodialyse et dialyse peritonéale: analyse comparative des rapports cout-efficacité. Montréal: Conseil d'Évaluation des Technologies de la Santé du Québec; 1998.

149. Rafftery J. NICE: faster access to modern treatments? Analysis of guidance on health technologies. BMJ 2001;323:1300-3.

150. Sharples LD, Briggs A, Caine N, McKenna M, Buxton M. A model for analyzing the cost of main 
clinical events after cardiac transplantation. Transplantation 1996;62:615-21.

151. Caine N, Sharples L, Buxton M, Briggs A, McKenna M. The development of a cost model to reflect the main clinical events post cardiac transplantation. HERG Research Report No. 21. Uxbridge: Health Economics Research Group, Brunel University; 1995.

152. Fenn P. The analysis of censored treatment cost data in economic evaluation. Med Care 1995; 33:851-63.

153. Bramstedt KA. Left ventricular assist devices and the slippery slope of ageism. Int J Cardiol 2001; 81:201-3.

154. Department of Health. NHS Reference Costs 2003. URL: http://www.dh.gov.uk/assetRoot/04/06/98/01/ 04069801.xls (accessed 8 March 2004).

155. Assist devices offer heart patients independence. Case Manage Advisor 2000;11:32-6.

156. Netten A, Curtis L. Unit costs of health and social care 2003. Public Social Services Research Unit (PSSRU): University of Kent, Canterbury; 2003.

157. World value of the Euro and Dollar. Wall Street J Eur. 9 January 2004.

158. Stewart S, Jenkins A, Buchan S, McGuire A, Capewell S, McMurray JJJV. The current cost of heart failure to the National Health Service in the UK. Eur J Heart Fail 2002;4:361-71.

159. MicroMed Technology. Submission on the clinical effectiveness, cost effectiveness and costs of the MicroMed DeBakey VAD. Houston, TX: MicroMed Technology; 2003.

160. Jarvik Heart, Submission on the clinical effectiveness and costs of the Jarvik 2000 LVAD. New York: Jarvik Heart; 2003.

161. Franic D, Pathak D. Effect of including (versus excluding) fates worse than death on utility measurement. Int J Technol Assess Health Care 2003;19:347-61.

162. British Heart Foundation. The costs of heart failure to the NHS, 2000. URL: http://www.heartstats.org/datapage.asp?id $=817$ (accessed 8 March 2004).

163. Berry C, Murdoch DR, McMurray JJ. Economics of chronic heart failure. Eur J Heart Fail 2001; 3:283-91.

164. UK Transplant. URL: http://www.uktransplant.org.uk/ statistics/yearly_statistics/yearly_heart_ statistics.htm

165. Anyanwu A, Treasure T. Prognosis after heart transplantation. BMJ 2003;326:510-1.

166. Gronda E, Vitali E. Left ventricle assist systems: a possible alternative to heart transplantation for heart failure patients? Patient selection, techniques and benefit. Eur J Heart Fail 1999;1:319-25.

167. Stein, R. New way to mend broken hearts. Wall Street J Eur 7-9 November 2003; A9.

168. Wong PS, Davidsson GK, Timeyin J, Warren A, Watson DJ, Vincent R, et al. Heart failure in patients admitted to hospital: mortality is still high. Eur J Intern Med 2002;13:304-10.

169. Brazier J, Roberts J, Deverill M. The estimation of a preference-based measure of health from the SF-36.J Health Econ 2002;21:149-58.

170. Kirsch J, McGuire A. Establishing health state valuations for disease specific states: an example from heart disease. Health Econ 2000;9: $149-58$.

171. Waugh N, Scott D. How should different life expectancies be valued? BMJ 1998;316:1316.

172. Billingham L, Abrams K, Jones D. Methods for the analysis of quality-of-life and survival data in health technology assessment. Health Technol Assess 1999;3(10).

173. Rydén L. Re-use of devices in cardiology. Proceedings from a Policy Conference at the European Heart House, 5-6 February, 1998. Eur Heart J 1998;19:1628-31.

174. Egger M, Davey Smith G, Altman D. Systematic reviews in health care: meta-analysis in context. London: BMJ Publishing; 2001.

175. Yacoub M, Tansley P, Birks E, Hipkin M, Hardy J, Bowles $\mathrm{C}$, et al. Interim results of left ventricular assist device combination therapy for inducing clinical and haemodynamic recovery of end stage dilated cardiomyopathy. Circulation 2002;106:2995

176. NHS Centre for Reviews and Dissemination. URL: http://agatha.york.ac.uk/darehp.htm (accessed 2000).

177. NHS Centre for Reviews and Dissemination. Undertaking systematic reviews of research on effectiveness: CRD guidelines for those carrying out or commissioning reviews. CRD Report 4. York: NHS Centre for Reviews and Dissemination, University of York; 1999.

178. Nuijten M, Pronk M, Brorens M, Hekster Y, Lockefeer J, de Smet P, et al. A reporting format for economic evaluation - Part II: focus on modelling studies. Pharmacoeconomics 1998; 14:259-68.

179. Jaski BE, Branch KR, Adamson R, Peterson KL, Gordon JB, Hoagland PM, et al. Exercise hemodynamics during long-term implantation of a left ventricular assist device in patients awaiting heart transplantation. J Am Coll Cardiol 1993; 22:1574-80. 
180. Centre for Health Economics. Kind P, Hardman G, Macran S. UK population norms for EQ-5D. Discussion Paper No. 172. York: Centre for Health Economics, University of York; 1999.

181. Beck J, Kassirer J, Pauker S. A convenient approximation of life expectancy (the DEALE). 1) Validation of the method, and 2) use in medical decision making. Am J Med 1982;73:883-97.

182. Hammond EH, Hansen JK, Spencer LS, Jensen A, Yowell RL. Immunofluorescence of endomyocardial biopsy specimens; methods and interpretation. J Heart Lung Transplant 1993;12:S113-24.

183. Billingham ME, Cary NR, Hammond ME, Kennitz J, Marboe C, McCallister HA, et al. A working formulation for the standardization of nomenclature in the diagnosis of heart and lung rejection: heart rejection study group. J Heart Transplant 1990;9:587-93.

184. Matsuwaka R, Matsuda H, Kaneko M, Miyamoto Y, Masai T, Nakatani T, et al. Overseas transport of a patient with an extracorporeal left ventricular assist device. Ann Thorac Surg 1995;59:522-3.
185. Noon GP, Hetzer R, Loebe M, Wolner E, Wieselthaler G, Schima H, et al. Clinical experience with the DeBakey $\operatorname{VAD}(\mathrm{TM})$ axial flow pump. Cardiovasc Eng 2000;5:30-2.

186. Noon GP, Ball J-WJ, Papaconstantinou HT. Clinical experience with BioMedicus centrifugal ventricular support in 172 patients. Artif Organs 1995;19:756-60.

187. International Society for Heart and Lung Transplantation. Sixteenth annual data report. URL: http://www.ishlt.org/registry.html

188. Cloy MJ, Myers TJ, Stutts LA, Macris MP, Frazier OH. Hospital charges for conventional therapy versus left ventricular assist system therapy in heart transplant patients. ASAIO J 1995;41:M535-M539.

189. Fiser SM, Wood HE, Allaire PE, Long J, Diegel P, Long SM, et al. A long-term ventricular assist device utilizing a magnetic bearing system and implantable physiologic controller. Heart Surg Forum 2001;4:11-2. 
This version of HTA monograph volume 9 , number 45 does not include the 198 pages of appendices. This is to save download time from the HTA website.

The printed version of this monograph also excludes the appendices.

$\underline{\text { View/download the appendices ( } 880 \text { kbytes). }}$ 



\section{Health Technology Assessment Programme}

\section{Members}

\section{Chair,}

Professor Tom Walley,

Director, NHS HTA Programme,

Department of Pharmacology \&

Therapeutics,

University of Liverpool

\section{Prioritisation Strategy Group}

Dr Ron Zimmern, Director, Public Health Genetics Unit, Strangeways Research

Laboratories, Cambridge

\section{HTA Commissioning Board}

\section{Members}

Programme Director,
Professor Tom Walley,

Director, NHS HTA Programme,

Department of Pharmacology \&

Therapeutics,

University of Liverpool

Chair,

Professor Jon Nicholl,

Director, Medical Care Research

Unit, University of Sheffield,

School of Health and Related

Research

\section{Deputy Chair,}

Professor Jenny Hewison,

Professor of Health Care

Psychology, Academic Unit of

Psychiatry and Behavioural

Sciences, University of Leeds

School of Medicine

Dr Jeffrey Aronson

Reader in Clinical

Pharmacology, Department of

Clinical Pharmacology,

Radcliffe Infirmary, Oxford

Professor Deborah Ashby,

Professor of Medical Statistics,

Department of Environmental

and Preventative Medicine,

Queen Mary University of

London
Professor Ann Bowling, Professor of Health Services

Research, Primary Care and

Population Studies,

University College London

Dr Andrew Briggs, Public Health Career Scientist, Health

Economics Research Centre,

University of Oxford

Professor John Cairns, Professor of Health Economics, Public Health Policy, London School of Hygiene and Tropical Medicine, London

Professor Nicky Cullum, Director of Centre for Evidence Based Nursing, Department of Health Sciences, University of York

Mr Jonathan Deeks, Senior Medical Statistician, Centre for Statistics in Medicine, University of Oxford

Dr Andrew Farmer, Senior Lecturer in General Practice, Department of Primary Health Care,

University of Oxford
Professor Fiona J Gilbert, Professor of Radiology, Department of Radiology, University of Aberdeen

Professor Adrian Grant, Director, Health Services Research Unit, University of Aberdeen

Professor F D Richard Hobbs, Professor of Primary Care \& General Practice, Department of Primary Care \& Genera Practice, University of Birmingham

Professor Peter Jones, Head of Department, University Department of Psychiatry, University of Cambridge

Professor Sallie Lamb, Professor of Rehabilitation, Centre for Primary Health Care, University of Warwick

Professor Stuart Logan, Director of Health \& Social Care Research, The Peninsula Medical School, Universities of Exeter \& Plymouth
Dr Linda Patterson, Consultant Physician, Department of Medicine, Burnley General Hospital

Professor Ian Roberts, Professor of Epidemiology \& Public Health, Intervention Research Unit, London School of Hygiene and Tropical Medicine

Professor Mark Sculpher, Professor of Health Economics, Centre for Health Economics, Institute for Research in the Social Services, University of York

Dr Jonathan Shapiro, Senior Fellow, Health Services Management Centre, Birmingham

Ms Kate Thomas, Deputy Director, Medical Care Research Unit, University of Sheffield

Ms Sue Ziebland, Research Director, DIPEx, Department of Primary Health Care, University of Oxford, Institute of Health Sciences 


\section{Diagnostic Technologies \& Screening Panel}

Members

Chair,

Dr Ron Zimmern, Director of the Public Health Genetics Unit,

Strangeways Research

Laboratories, Cambridge

Ms Norma Armston,

Lay Member, Bolton

Professor Max Bachmann

Professor of Health

Care Interfaces,

Department of Health

Policy and Practice,

University of East Anglia

Professor Rudy Bilous

Professor of Clinical Medicine \&

Consultant Physician,

The Academic Centre,

South Tees Hospitals NHS Trust

Dr Paul Cockcroft,

Consultant Medical

Microbiologist and Clinical

Director of Pathology,

Department of Clinical

Microbiology, St Mary's

Hospital, Portsmouth
Professor Adrian K Dixon,

Professor of Radiology,

University Department of

Radiology, University of

Cambridge Clinical School

Dr David Elliman,

Consultant Paediatrician/

Hon. Senior Lecturer,

Population Health Unit,

Great Ormond St. Hospital,

London

Professor Glyn Elwyn,

Primary Medical Care

Research Group,

Swansea Clinical School,

University of Wales Swansea

Mr Tam Fry, Honorary

Chairman, Child Growth

Foundation, London

Dr Jennifer J Kurinczuk,

Consultant Clinical

Epidemiologist

National Perinatal

Epidemiology Unit, Oxford
Dr Susanne M Ludgate, Medical Director, Medicines \&

Healthcare Products Regulatory

Agency, London

Professor William Rosenberg, Professor of Hepatology, Liver Research Group, University of Southampton

Dr Susan Schonfield, Consultan in Public Health, Specialised Services Commissioning North West London, Hillingdon Primary Care Trust

Dr Phil Shackley, Senior Lecturer in Health Economics, School of Population and Health Sciences, University of Newcastle upon Tyne

Dr Margaret Somerville, PMS Public Health Lead, Peninsula Medical School, University of Plymouth

Dr Graham Taylor, Scientific Director \& Senior Lecturer, Regional DNA Laboratory, The Leeds Teaching Hospitals
Professor Lindsay Wilson Turnbull, Scientific Director, Centre for MR Investigations \& YCR Professor of Radiology, University of Hull

Professor Martin J Whittle, Associate Dean for Education, Head of Department of Obstetrics and Gynaecology, University of Birmingham

Dr Dennis Wright, Consultant Biochemist \& Clinical Director, Pathology \& The Kennedy Galton Centre,

Northwick Park \& St Mark's Hospitals, Harrow

\section{Pharmaceuticals Panel}

Members

\section{Chair,}

Dr John Reynolds, Chair Division A, The John Radcliffe

Hospital, Oxford Radcliffe Hospitals NHS Trust

Professor Tony Avery,

Head of Division of Primary

Care, School of Community

Health Services, Division of

General Practice, University of

Nottingham

Ms Anne Baileff, Consultant

Nurse in First Contact Care,

Southampton City Primary Care

Trust, University of

Southampton

Professor Stirling Bryan,

Professor of Health Economics,

Health Services

Management Centre,

University of Birmingham
Mr Peter Cardy, Chief

Executive, Macmillan Cancer Relief, London

Professor Imti Choonara, Professor in Child Health, Academic Division of Child Health, University of

Nottingham

Dr Robin Ferner, Consultant Physician and Director, West Midlands Centre for Adverse Drug Reactions, City Hospital NHS Trust, Birmingham

Dr Karen A Fitzgerald,

Consultant in Pharmaceutical Public Health, National Public Health Service for Wales,

Cardiff

Mrs Sharon Hart, Head of DTB Publications, Drug $\mathcal{E}$ Therapeutics Bulletin, London
Dr Christine Hine, Consultant in Public Health Medicine, South Gloucestershire Primary Care Trust

Professor Stan Kaye,

Cancer Research UK

Professor of Medical Oncology, Section of Medicine,

The Royal Marsden Hospital, Sutton

Ms Barbara Meredith,

Lay Member, Epsom

Dr Andrew Prentice, Senior

Lecturer and Consultant

Obstetrician \& Gynaecologist,

Department of Obstetrics \&

Gynaecology, University of

Cambridge

Professor Jan Scott, Professor of Psychological Treatments, Institute of Psychiatry,

University of London

Mrs Katrina Simister, Assistant Director New Medicines, National Prescribing Centre, Liverpool

Dr Richard Tiner, Medical Director, Medical Department, Association of the British Pharmaceutical Industry, London

Dr Helen Williams,

Consultant Microbiologist, Norfolk \& Norwich University Hospital NHS Trust

Dr Frances Rotblat, CPMP

Delegate, Medicines \&

Healthcare Products Regulatory

Agency, London 


\section{Therapeutic Procedures Panel}

Members

Chair,

Professor Bruce Campbell,

Consultant Vascular and General Surgeon, Department

of Surgery, Royal Devon \&

Exeter Hospital

Dr Aileen Clarke,

Reader in Health Services

Research, Public Health \&

Policy Research Unit, Barts \&

the London School of Medicine

\& Dentistry, London

Dr Matthew Cooke, Reader in A\&E/Department of Health

Advisor in A\&E, Warwick

Emergency Care and

Rehabilitation, University of

Warwick
Dr Carl E Counsell, Clinical

Senior Lecturer in Neurology,

Department of Medicine and

Therapeutics, University of

Aberdeen

Ms Amelia Curwen, Executive Director of Policy, Services and Research, Asthma UK, London

Professor Gene Feder, Professor of Primary Care R\&D,

Department of General Practice and Primary Care, Barts \& the London, Queen Mary's School of Medicine and Dentistry,

London

Professor Paul Gregg,

Professor of Orthopaedic

Surgical Science, Department of

General Practice and Primary

Care, South Tees Hospital NHS

Trust, Middlesbrough

Ms Bec Hanley, Co-Director,

TwoCan Associates,

Hurstpierpoint
Ms Maryann L Hardy,

Lecturer, Division of

Radiography, University of

Bradford

Professor Alan Horwich, Director of Clinical R\&D,

Academic Department of

Radiology, The Institute of

Cancer Research

London

Dr Simon de Lusignan,

Senior Lecturer,

Primary Care Informatics,

Department of Community

Health Sciences,

St George's Hospital Medical

School, London

Professor Neil McIntosh,

Edward Clark Professor of

Child Life \& Health,

Department of Child Life \&

Health, University of

Edinburgh
Professor James Neilson,

Professor of Obstetrics and

Gynaecology, Department of

Obstetrics and Gynaecology,

University of Liverpool

Dr John C Pounsford,

Consultant Physician,

Directorate of Medical Services,

North Bristol NHS Trust

Karen Roberts, Nurse

Consultant, Queen Elizabeth

Hospital, Gateshead

Dr Vimal Sharma, Consultant

Psychiatrist/Hon. Senior Lecturer,

Mental Health Resource Centre,

Cheshire and Wirral Partnership

NHS Trust, Wallasey

Dr L David Smith, Consultant Cardiologist, Royal Devon \&

Exeter Hospital

Professor Norman Waugh, Professor of Public Health, Department of Public Health, University of Aberdeen 


\section{Expert Advisory Network}

Members

Professor Douglas Altman, Director of CSM \& Cancer Research UK Med Stat Gp,

Centre for Statistics in Medicine, University of Oxford, Institute of Health Sciences, Headington, Oxford

Professor John Bond, Director, Centre for Health Services Research, University of Newcastle upon Tyne, School of Population \& Health Sciences, Newcastle upon Tyne

Mr Shaun Brogan,

Chief Executive, Ridgeway

Primary Care Group, Aylesbury

Mrs Stella Burnside OBE Chief Executive, Office of the Chief Executive. Trus Headquarters, Altnagelvin Hospitals Health \& Social Services Trust, Altnagelvin Area Hospital, Londonderry

Ms Tracy Bury, Project Manager, World Confederation for Physical Therapy, London

Professor Iain T Cameron, Professor of Obstetrics and Gynaecology and Head of the School of Medicine, University of Southampton

Dr Christine Clark, Medical Writer \& Consultant Pharmacist, Rossendale

Professor Collette Clifford, Professor of Nursing \& Head of Research, School of Health Sciences, University of Birmingham, Edgbaston, Birmingham

Professor Barry Cookson, Director, Laboratory of

Healthcare Associated Infection, Health Protection Agency, London

Professor Howard Cuckle, Professor of Reproductive Epidemiology, Department of Paediatrics, Obstetrics \& Gynaecology, University of Leeds

Dr Katherine Darton, Information Unit, MIND The Mental Health Charity, London

Professor Carol Dezateux, Professor of Paediatric Epidemiology, London
Mr John Dunning, Consultant Cardiothoracic Surgeon, Cardiothoracic Surgical Unit, Papworth Hospital NHS Trust, Cambridge

Mr Jonothan Earnshaw, Consultant Vascular Surgeon, Gloucestershire Royal Hospital, Gloucester

Professor Martin Eccles,

Professor of Clinical

Effectiveness, Centre for Health Services Research, University of Newcastle upon Tyne

Professor Pam Enderby, Professor of Community Rehabilitation, Institute of General Practice and Primary Care, University of Sheffield

Mr Leonard R Fenwick, Chief Executive, Newcastle upon Tyne Hospitals NHS Trust

Professor David Field,

Professor of Neonatal Medicine, Child Health, The Leicester Royal Infirmary NHS Trust

Mrs Gillian Fletcher, Antenatal Teacher \& Tutor and President, National Childbirth Trust, Henfield

Professor Jayne Franklyn, Professor of Medicine, Department of Medicine, University of Birmingham, Queen Elizabeth Hospital, Edgbaston, Birmingham

Ms Grace Gibbs, Deputy Chief Executive, Director for Nursing, Midwifery \& Clinical Support Services, West Middlesex University Hospital, Isleworth

Dr Neville Goodman, Consultant Anaesthetist, Southmead Hospital, Bristol

Professor Alastair Gray, Professor of Health Economics, Department of Public Health, University of Oxford

Professor Robert E Hawkins, CRC Professor and Director of Medical Oncology, Christie CRC Research Centre, Christie Hospital NHS Trust, Manchester

Professor Allen Hutchinson, Director of Public Health \& Deputy Dean of ScHARR, Department of Public Health, University of Sheffield
Dr Duncan Keeley, General Practitioner (Dr Burch \& Ptnrs), The Health Centre, Thame

Dr Donna Lamping, Research Degrees Programme Director \& Reader in Psychology, Health Services Research Unit, London School of Hygiene and Tropical Medicine, London

Mr George Levvy,

Chief Executive, Motor

Neurone Disease Association,

Northampton

Professor James Lindesay, Professor of Psychiatry for the Elderly, University of Leicester, Leicester General Hospital

Professor Julian Little, Professor of Human Genome Epidemiology, Department of Epidemiology \& Community Medicine, University of Ottawa

Professor Rajan Madhok, Medical Director \& Director of Public Health, Directorate of Clinical Strategy \& Public Health, North \& East Yorkshire \& Northern Lincolnshire Health Authority, York

Professor David Mant,

Professor of General Practice, Department of Primary Care, University of Oxford

Professor Alexander Markham, Director, Molecular Medicine Unit, St James's University Hospital, Leeds

Dr Chris McCall,

General Practitioner, The Hadleigh Practice, Castle Mullen

Professor Alistair McGuire, Professor of Health Economics, London School of Economics

Dr Peter Moore, Freelance Science Writer, Ashtead

Dr Sue Moss, Associate Director, Cancer Screening Evaluation Unit, Institute of Cancer Research, Sutton

Mrs Julietta Patnick,

Director, NHS Cancer Screening

Programmes, Sheffield

Professor Tim Peters,

Professor of Primary Care Health Services Research, Academic Unit of Primary Health Care, University of Bristol
Professor Chris Price,

Visiting Chair - Oxford, Clinica Research, Bayer Diagnostics

Europe, Cirencester

Professor Peter Sandercock, Professor of Medical Neurology, Department of Clinical Neurosciences, University of Edinburgh

Dr Eamonn Sheridan, Consultant in Clinical Genetics, Genetics Department, St James's University Hospital, Leeds

Dr Ken Stein,

Senior Clinical Lecturer in

Public Health, Director,

Peninsula Technology

Assessment Group,

University of Exeter

Professor Sarah Stewart-Brown, Professor of Public Health, University of Warwick, Division of Health in the Community Warwick Medical School, LWMS, Coventry

Professor Ala Szczepura, Professor of Health Service Research, Centre for Health Services Studies, University of Warwick

Dr Ross Taylor,

Senior Lecturer, Department of General Practice and Primary Care, University of Aberdeen

Mrs Joan Webster, Consumer member, HTA Expert Advisory Network 



\section{Feedback}

The HTA Programme and the authors would like to know your views about this report.

The Correspondence Page on the HTA website (http://www.ncchta.org) is a convenient way to publish your comments. If you prefer, you can send your comments

to the address below, telling us whether you would like us to transfer them to the website.

\section{We look forward to hearing from you.}

The National Coordinating Centre for Health Technology Assessment,

Mailpoint 728, Boldrewood,

University of Southampton,

Southampton, SOI6 7PX, UK.

Fax: +44 (0) $2380595639 \quad$ Email: hta@soton.ac.uk

http://www.ncchta.org 